\title{
Mineral Resources of
}

\section{the Emigrant Basin}

\section{Primitive Area,}

\section{California}

By E. W. TOOKER, H. T. MORRIS, U.S. Geological Survey, and PAUL V. FIllo, U.S. Bureau of Mines

With a section on GEOPHYSICAL STUDIES

By H. W. OLIVER, U.S. Geological Survey

STUDIES RELATED TO WILDERNESS-PRIMITIVE AREAS

G E O L O G I C A L S U R V E Y B U L L E T I N 1261-G

An evaluation of the mineral

potential of a part of the

central Sierra Nevada

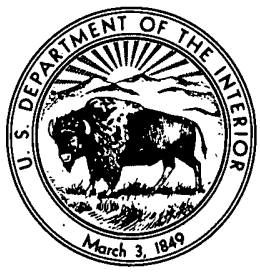




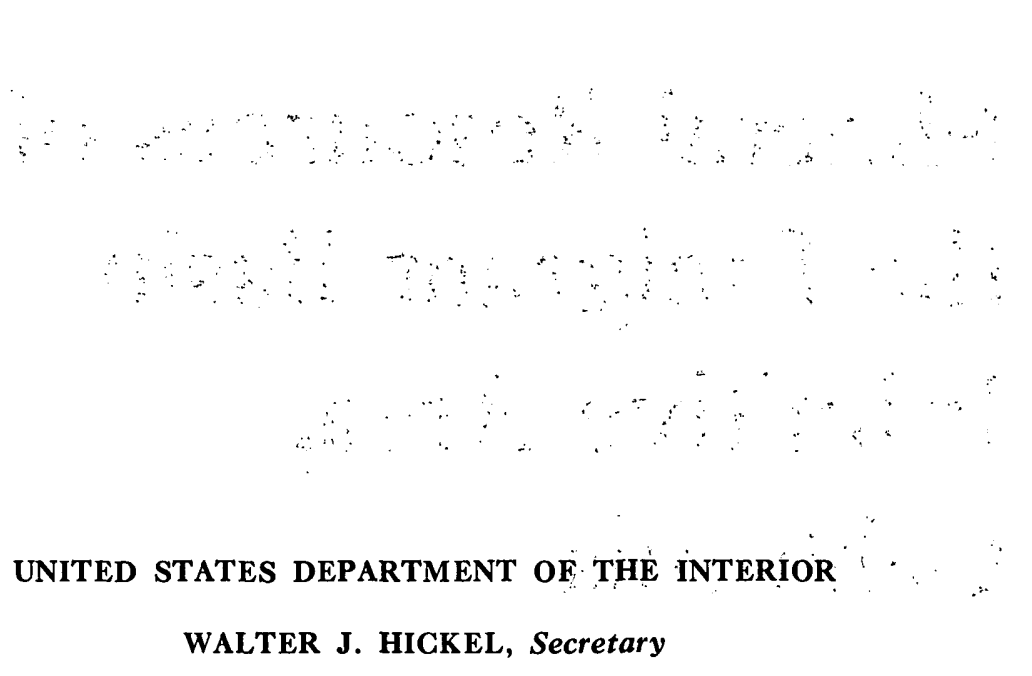

WALTER J. HIGKEL, Secretary

GEOLOGICAL SUTVEY:

William T. Pecora, Director

$a x$

$\rightarrow \rightarrow$

欧

$\boldsymbol{r}:$ 


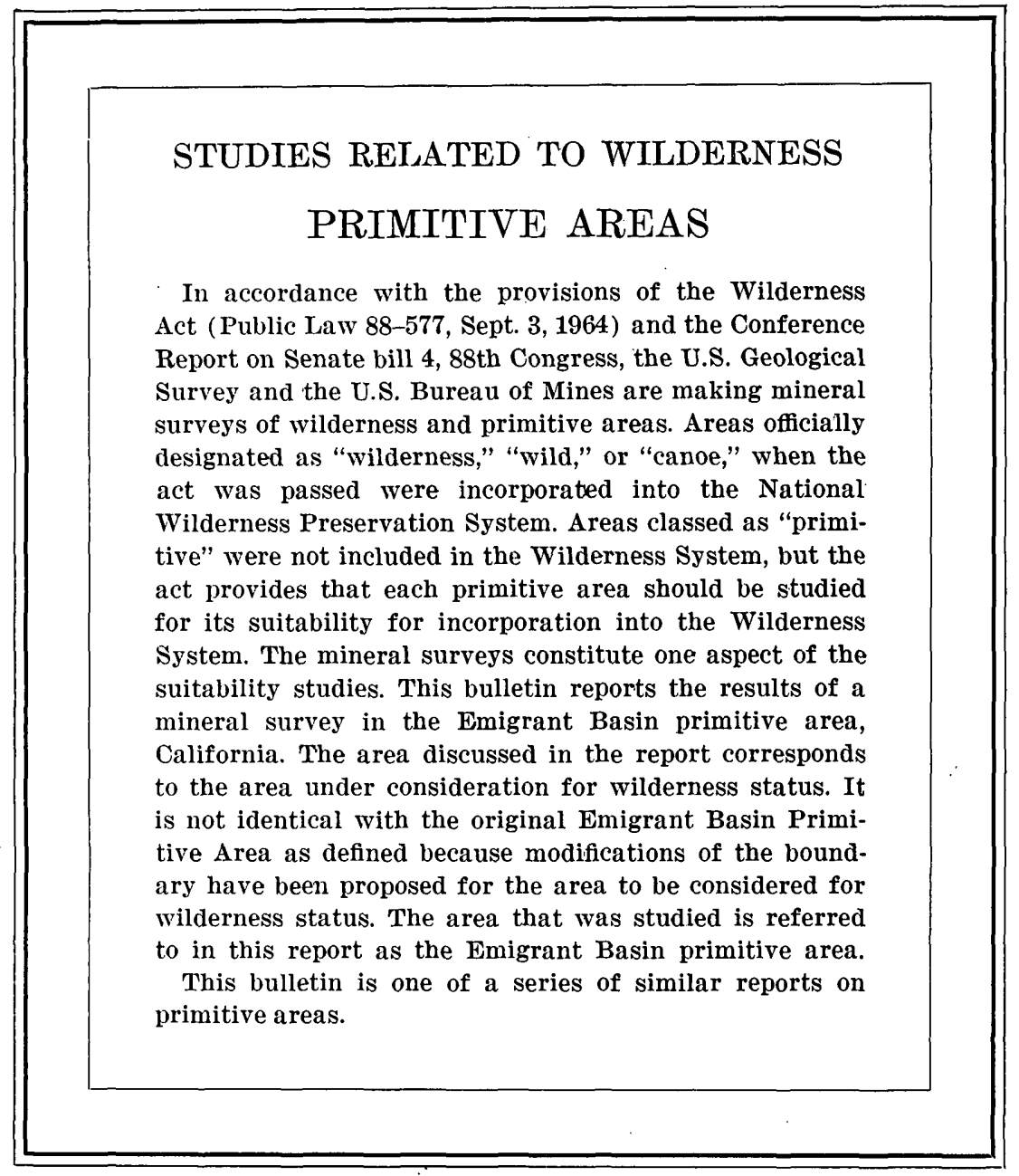


$-$

$b$

$a$

col

2

$+$

n:

-6?

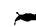

$-$

aो

$+4$

2

.

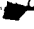

$-$

2

$+$

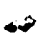

$b$

$\downarrow$

$r$

43

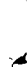

$-$

67

$A$

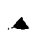




\section{CONTENTS}

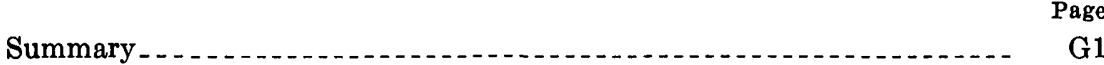

Introduction . .

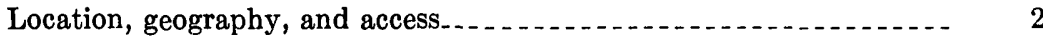

Fieldwork and authorship of report

Acknowledgments.

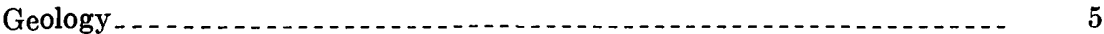

Previous geologic studies.

Present study

Geologic setting

Rock units.......... 10

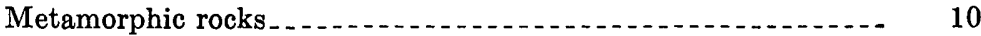

Igneous rocks. .

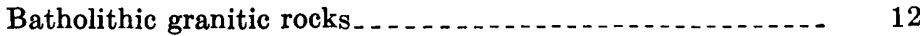

Dike rocks and plugs.

Volcanic rocks.

Unconsolidated deposits _.

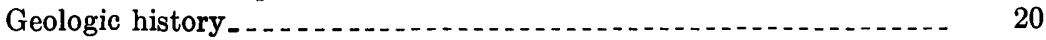

Ore deposits in the region

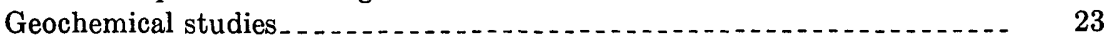

General objectives and sampling procedures................ 23

Limitations of the geochemical data

Geochemistry of rocks and unconsolidated deposits

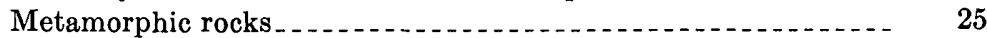

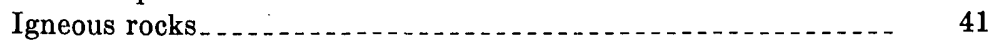

Granitic rocks of the Sierra Nevada batholith

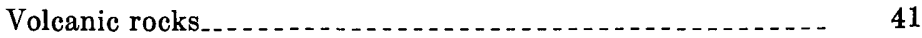

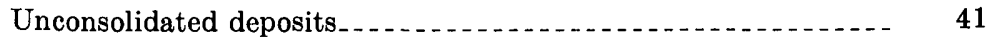

Geochemistry of hydrothermally altered areas................. 42

Hydrothermally altered zones in granitic rocks............ 42

Hydrothermally altered zones in volcanic rocks.............. 43

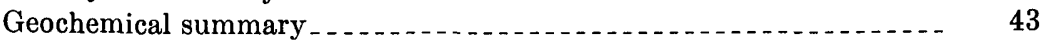

Geophysical studies, by H. W. Oliver.

Methods of evaluation

Description of magnetic field.

Magnetic properties of geologic units... 45

Interpretation of magnetic data.

Economic significance of magnetic data

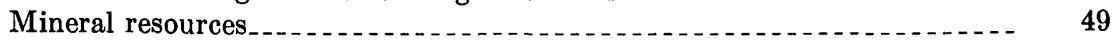

Nearby productive deposits...... 49

Juniper mine

Prospect on Silver Mine Creek 51

Prospects near Dorothy Lake.

Mines and prospects within the primitive area.... 52

Mining claims 
Mineral resources-Continued . Page

Tungsten deposits..... G53

Whittle property $\ldots$

Jan Cavanaugh claim

Francis Finley claim

Garnet claim .

Tamarack claim _...... 58

Montezuma mine

Bigelow group.

Buckskin group.

Eleven Kids claim . 64

Snow Lake claims

Mineral potential of the primitive area

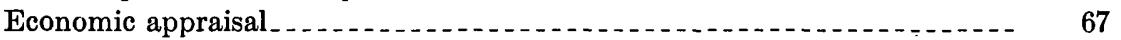

References cited........ 68

Plate 1. Generalized geologic and magnetic intensity map and sections of the Emigrant Basin primitive area, California _..... In pocket

Figure 1. Map showing location of the Emigrant Basin primitive area,

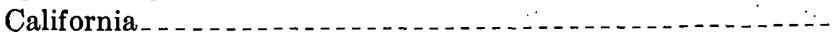

2. Photograph of Shallow Lake, central part; Emigrant Basin

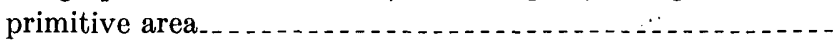

3. Map showing geologic traverses, Emigrant Basin primitive area, California ... . . . . . . . . . . . . . . . . . . . . . . . .

4. Map showing location and type of analyzed samples, Emigrant Basin primitive area, California

5-10. Photographs showing:

5: Typical tightly folded epidote-garnet tactite lens in metasedimentary rocks, Bigelow Peak roof pendant.-

6. Steeply dipping metasedimentary rock intruded by granitic rocks in Toms Canyon septum

7. Dioritic xenolith in porphyritic granitic rock cut by an aplite dike in Louse Canyon.................. 14

8. East Flange Rock erosion valley cut in granite _._.

9. Typical glacial-scoured area near Emigrant Lake__._. $\quad 19$

10. U-shaped Kennedy Valley.................. 20

11. Map showing mining and prospecting sites in Emigrant Basin primitive area.

12. Map showing claims, surface development, and sample locations in the western part of the Whittle property......-

13. Photograph of western part of the Whittle property showing gravity concentrating mill

14. Map and section of the geology of the Montezuma mine, Tuolumne County, Calif 
Figure 15. Geologic map of part of the Bigelow group of claims, Tuolumne

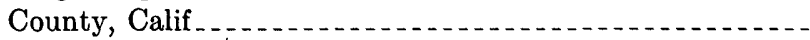

16. Map showing surface geology and sample locations, Bigelow

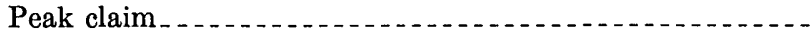
17-19. Photographs of:

17. Discovery pit, Buckskin No. 2 claim

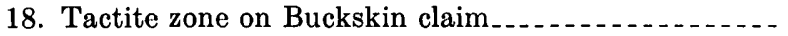

19. Face of prospect pit, Buckskin No. 2 claim

\section{T A B L E S}

TABLE 1. Chemical analyses of granitic rock units in Emigrant Basin primitive area..................................

2. Volcanic rocks in Emigrant Basin primitive area........

3. Chemical analyses of two volcanic rocks, Emigrant Basin primitive area............................................

4. Analyses of samples from the Emigrant Basin primitive

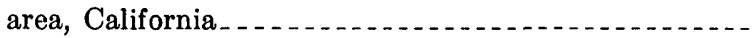

5. Abundance of selected metals in rocks of the Emigrant Basin primitive area compared with the abundance of these metals in similar rocks in the world.

6. Magnetic susceptibilities, remanent magnetizations, Koenigsberger ratios, and densities of selected rock samples. . .

7. Dependence of the susceptibility contrast required to produce the Emigrant Lake anomaly on the assumed depth extent of the magnetic mass ....................... 
$-$

$\downarrow$

$\alpha$

$-1$

$\gamma$

$+$

N

4

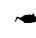

-

in

at

i

$\dot{r}$

4

4

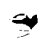

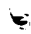

rr.

$\rightarrow$

$+$

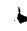

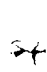

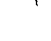

a-

-

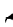

L. $-1$ 


\title{
STUDIES RELATED TO WILDERNESS-PRIMITIVE AREAS
}

\section{MINERAL RESOURCES OF THE EMIGRANT BASIN PRIMITIVE AREA, CALIFORNIA}

\author{
By E. W. Tooker and H. T. Morris, U.S. Geological Survey, and \\ PaUl V. Fillo, U.S. Bureau of Mines
}

\section{SUMMARY}

The Emigrant Basin primitive area, California, which was examined during 1967 and 1968 by the U.S. Geological Survey and the U.S. Bureau of Mines under the provisions of the Wilderness Act of 1964, lies immediately west of the backbone divide of the central Sierra Nevada and adjoins the northern boundary of Yosemite National Park. It consists of approximately 180 square miles of scenic lakes, streams, and rugged mountains that are largely underlain by batholithic granitic rocks of Mesozoic age. Scattered remnants of deformed and metamorphosed rocks of Paleozoic(?) age occur within the batholith. Aplitic, pegmatitic, dioritic, and gabbroic dikes, and other intrusive bodies, cut both the granitic and metamorphic rocks, and extrusive volcanic rocks of late Cenozoic age unconformably overlie the granitic rocks in the northern part of the area. Quaternary glaciation has exposed most of the bedrock leaving only sparse moraines, and post-glacial streams have deposited thin patches of alluvium.

The objective of the study was to evaluate the known and potential mineral resources of the area. The mineral survey included geologic mapping, geochemical sampling, geophysical surveying, and close study of known mineral deposits and of areas considered favorable for the occurrence of mineral deposits. The distribution and structure of rock units is shown on a geologic map $(1: 62,500$ scale) and cross sections. The geochemical studies of the area are based on analyses of nearly 400 samples of fresh and altered rocks, stream sediment, soil, and vein material. Aeromagnetic profiles spaced 1 mile apart are the basis for a magnetic map, which is superimposed on the geologic map. Small uranium, base and precious metal, and tungsten deposits are known to exist nearby, but only tungsten deposits occur in the primitive area itself. In general, the known tungsten deposits are small, scattered, and discontinuous, and are restricted to the contact zone of the roof pendants and the granitic rocks. Several hydrothermally altered zones in granitic and volcanic rocks are present in the area, but no commercial mineral concentrations of ore minerals were found in them.

The economic appraisal is based on interpretation of geologic information, Tuolumne County claim records, a search of the literature and production records, interviews with claim owners, and on-site field inspection. It revealed that 44 recorded claims have been located for tungsten in the area of T. 4 N., R. 21 E.,

$368-4560-70-2$ 
Mount Diablo base line and meridian. One property, currently being developed, had 110 feet of underground workings in 1968 and a 100-ton-per-day mill to concentrate scheelite $\left(\mathrm{CaWO}_{4}\right)$; a small production is recorded. A second property is developed by 215 feet of underground workings, and it also has a small recorded production. Some prospecting activity was in progress elsewhere in the area at the time of the study.

The overall mineral resource potential of the primitive area is considered minimal; however, the possibility exists that additional physical exploration may disclose downward extensions of known deposits or new deposits. Such discoreries are most likely to be made in a small area in the southeastern part of the primitive area in limy roof-pendant rocks near contacts with the granitic rocks. The metal content of rocks elsewhere in the primitive area is generally within the average abundance level that characterizes nonmineralized regions. No Federal leasable mineral or mineral fuel reserve lands are included in the primitive area.

\section{INTRODUCTION}

\section{LOCATION, GEOGRAPHY, AND ACCESS}

The Emigrant Basin primitive area is a region of rugged peaks, glacial lakes, mountain meadows, and patches of conifer forest in the Stanislaus National Forest, Tuolumne County, Calif. It is an elongate area that trends northeast, is about 25 miles in length, and ranges from 3 to 11 miles in width. It contains about 115,000 acres (180 square miles), chiefly between long. $119^{\circ} 35^{\prime}$ and $119^{\circ} 55^{\prime} \mathrm{W}$. and lat. $38^{\circ} 00^{\prime}$ and $38^{\circ} 20^{\prime} \mathrm{N}$. (figs. 1 and 2 ). The southern boundary of the area follows part of the northern border of Yosemite National Park, and the northern boundary lies a short distance south of Sonora Pass.

The Emigrant Basin area is used primarily for outdoor recreation, and from late spring to early fall it is visited by large numbers of hikers, campers, and fishermen. In addition, it supports a minor amount of mining activity and is a summer forage area for cattle. The area receives heavy winter snowfall and much summer rain and is an important watershed. To increase water storage in the region and to facilitate uniform streamflow, many of the glacial lakes in the area have been enlarged inconspicuously by the construction of masonry and concrete dams.

The topography of the primitive area is relatively rugged although it is not as deeply dissected as the parts of the range both nonth and south. The northern third of the area is drained by the Middle Fork of the Stanislaus River and the southern two-thirds, by tributaries of the Tuolumne River. The elevation of the region ranges from 4,700 feet at Cherry Lake near the southwestern tip of the area to 11,570 feet at Leavitt Peak on the crest of the Sierra Nevada near the northeastern tip. Emigrant Pass, $61 / 2$ miles south of Leavitt Peak, at an elevation of about 9,650 feet, was the site of disastrous attempts to cross the Sierra Nevada by the Duckwall and Wash Train emigrant 


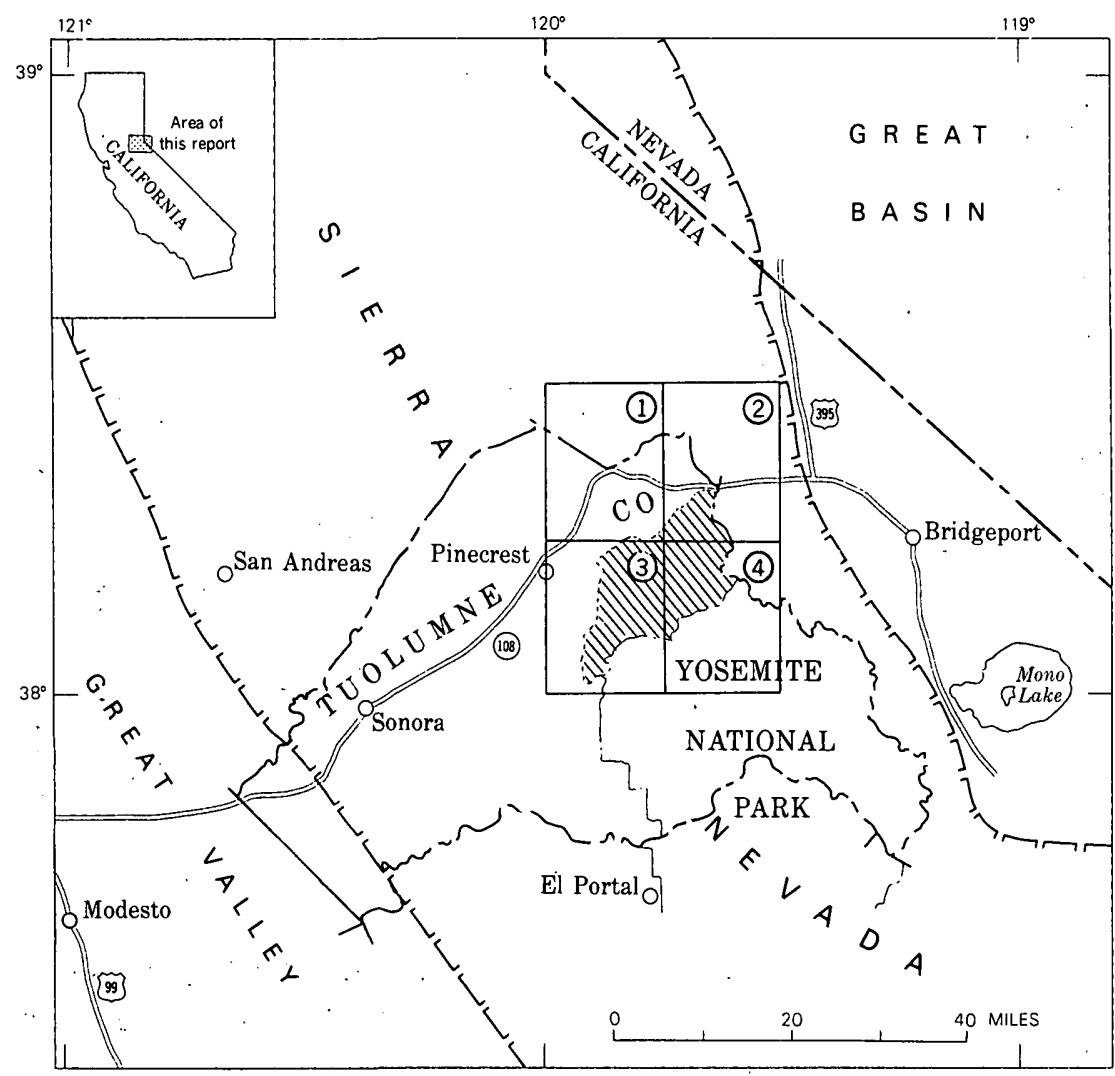

FIgUre 1.-Location of the Emigrant Basin primitive area, Tuolumne County, Calif. (shaded). Topographic quadrangle maps, scale 1:62,500, indicated are (1) Dardanelles Cone, (2) Sonora Pass, (3) Pinecrest, and (4) Tower Peak.

parties in October 1853 (Rensch and others, 1933, p. 502). Unseasonable snow and virtually impassable topography west of the crest stranded the emigrants, several of whom died of exposure and exhaustion before the survivors were guided out of danger by a relief party from Sonora.

\section{FIELDWORK AND AUTHORSHIP OF REPORT}

This report is the result of the combined efforts of personnel of the U.S. Geological Survey and the U.S. Bureau of Mines. In July, August, and September of 1967, E. W. Tooker and H. T. Morris, assisted by Michael Naney and Wayne Wurtsbaugh, examined the geology of the entire primitive area and collected samples for geochemical studies. Additional shorter field studies and mine and prospect examinations were made by these authors in October 1967 and in June 1968, in part in association with George O. Gates. Paul V. Fillo examined 


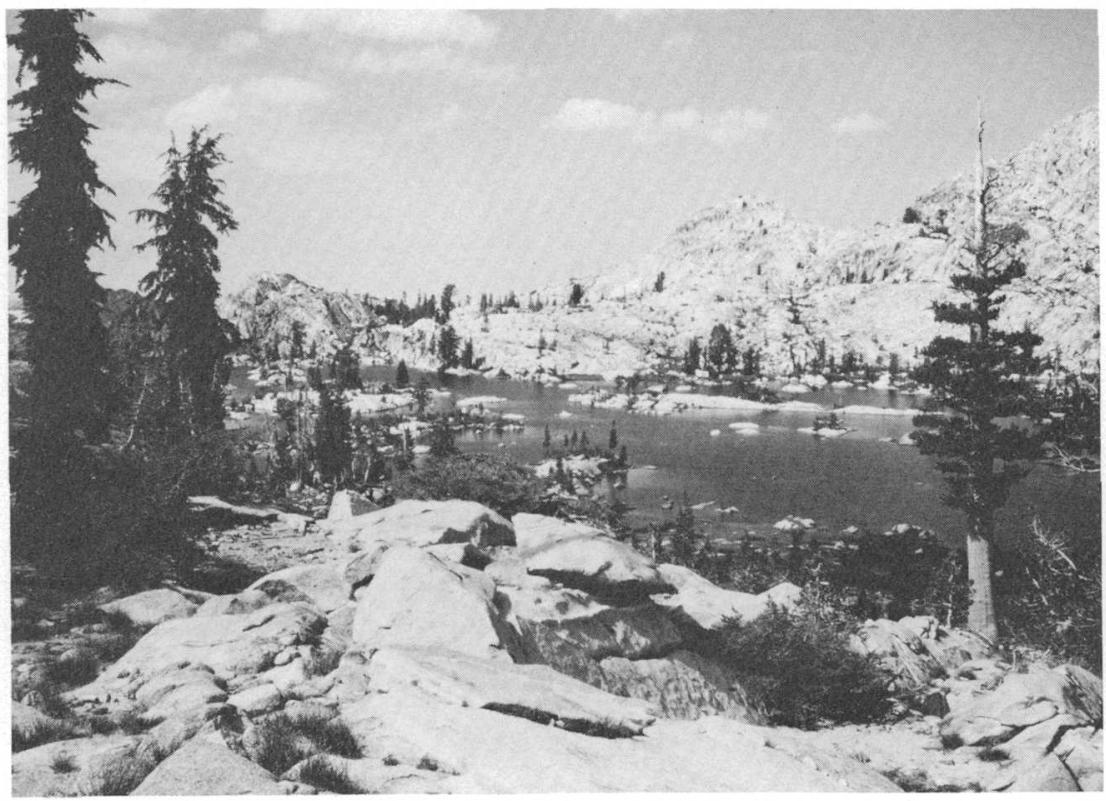

Figure 2.-Shallow Lake, Emigrant Basin primitive area.

the mines and prospects in July 1966, September 1967, and August 1968 , in part in association with officials of the U.S. Forest Service. The aeromagnetic survey was made in July 1968 and the data were reduced and evaluated and the section on geophysical studies was prepared later that year by $\mathrm{H}$. W. Oliver.

The parts of the report describing the geological and geochemical studies in the Emigrant Basin area were prepared by E. W. Tooker and H. T. Morris. Paul V. Fillo prepared most of the section on mines and prospects, and all three authors contributed to the sections on the mineral potential and economic appraisal of the primitive area. The validity of the data, conclusions, and recommendations contained in the report are accepted as the joint responsibility of all of its authors.

\section{ACKNOWLEDGMENTS}

Ian Campbell, Chief, California Division of Mines and Geology, and J. B. Koenig of his staff, who is working in part of the Emigrant Basin area, generously provided data concerning the geology and mineral development of the region. Clyde Wahrhaftig, Department of Geology, University of California, and D. B. Slemmons, Department of Geology, University of Nevada, both of whom are conducting continuing independent geologic research studies in the area, also provided information used in preparing the geologic map. In addition, data from preliminary studies of the National Aeronautics and Space 
Administration remote sensing test site area lying between Ruth Lake and Relief Peak were made available by D. B. Slemmons, Jack Quade, Peter Chapman, James Sjoberg, Malcolm Hibbard, and David Stern of the Mackay School of Mines, University of Nevada, Reno, Nev.

Other persons who generously provided information about the geology, mines, and prospecting activity include E. H. Boudreau, Sebastopol, Calif., V. W. Butorovich, Alameda, Calif., D. R. Whittle, Angels Camp, Calif., E. L. Dahl, Tuolumne, Calif., F. W. Hanson, Yerington, Nev., L. W. Osborn, Reno, Nev., and H. L. Valk, Oakdale, Calif., and H. K. Stager, D. C. Ross, and D. B. Tatlock of the U.S. Geological Survey.

In addition, we wish to thank M. M. Barnum, former Assistant Regional Forester, Joseph V. Flynn, William L. Johnson, W. C. Sanborn, and H. P. Turner of the U.S. Forest Service, San Francisco, Calif., and their field staffs in the Stanislaus National Forest, for their cooperation and aid, including the use of the Forest Service contract helicopter piloted by E. E. Northern. During several visits to the Cherry Creek mine, the late John Loring, who was general superintendent, cordially provided camping facilities, tours of the claims held by his company, and data concerning the grade and size of the tungsten deposits under development in the area of the Cherry Creek mine.

Joe Barnes, Oakdale, Calif., provided the field party with highly competent logistical and packing services, and his packer, Patrick Casey, was especially helpful because of his wide personal knowledge of the Emigrant Basin primitive area and because of his efficient and capable handling of the riding horses and pack animals. Reno Sardella of Kennedy Meadows, Calif., also facilitated the field studies by extending the use of his Dodge Ridge, Cherry Lake, and Kennedy Meadows pack station facilities.

\section{GEOLOGY}

\section{PREVIOUS GEOLOGIC STUDIES}

The generalized geology of the Emigrant Basin primitive area and adjacent areas in the Sierra Nevada, both to the south and north, is shown on the 1:250,000-scale Walker Lake sheet of the "Geologic Map of California," compiled by Koenig (1963). The geology of Emigrant Basin shown on this map is largely based on unpublished data by Clyde Wahrhaftig, D. B. Slemmons, and J. P. Koenig. F. L. Ransome published a report on the area in 1898. Recent geologic and geophysical studies of the central portion of the Sierra Nevada batholith immediately south of the area by Bateman, Clark, Huber, Moore, and Rinehart (1963), Bateman and Eaton (1967), and Bateman and Wahrhaftig (1966) also apply to the Emigrant Basin primitive area. 
'These detailed reports describe the general character and mode of emplacement of the various rocks and the geologic history. A recent report by Smith (1968) describes the mineral, textural, and chemical characteristics of intrusive rocks in Yosemite National Park. The Cenozoic volcanic activity in the Sierra Nevada region, mostly north of the primitive area, is discussed by Slemmons (1966), and the geomorphic development of the Yosemite National Park region, mostly south of the primitive area, has been summarized by Matthes (1930) and by Wahrhaftig (1962).

No known published reports describe the mineral deposits of the Emigrant Basin primitive area, although it was doubtlessly well prospected during the last 115 years. Prospectors must have explored the area during the gold rush of 1849 , and more recently, during the periods of accelerated mineral exploration attendant on World Wars I and II and during the uranium boom of the early 1950's. Wahrhaftig (1962, p. 35) describes old river channels containing auriferous gravels 30 to 50 miles west of the Emigrant Basin area, but no extensions of these deposits in the Emigrant Basin area have been reported. Similar pre-volcanic stream channels contain uranium deposits a short distance north of the primitive area in the drainage basins of Eagle and Niagara Creeks; these deposits have been studied by E. H. Boudreau (oral commun. from J. B. Koenig, 1967) and by personnel of the U.S. Geological Survey. Finally, the occurrence and production of tungsten and molybdenum minerals from a few shallow mines and prospects near Bigelow Peak and Dorothy Lake on the southeastern margin of the area are described in an open-file report by Lemmon, Bateman, Stagner, and Beatty (1943).

\section{PRESENT STUDY}

The objective of the present study is to evaluate the mineral potential of the Emigrant Basin primitive area. The present report responds to the following questions: (1) Does this primitive area have any economically significant mineral occurrences? (2) What kinds of mineral commodities are present and in what possible quantities and values do they occur? and (3) What parts of the area contain the most significant indications of mineral potential?

The principal field techniques used in making the study included observation of the geologic character of the terrain, reconnaissance geologic mapping, geochemical sampling, geophysical surveying, and detailed examination of mines, mining claims, and mineral prospects within the area. Horseback and foot traverses were made along all improved trails and along many stream drainages and ridge crests (fig. 3). The more rugged and inaccessible parts of the area were 


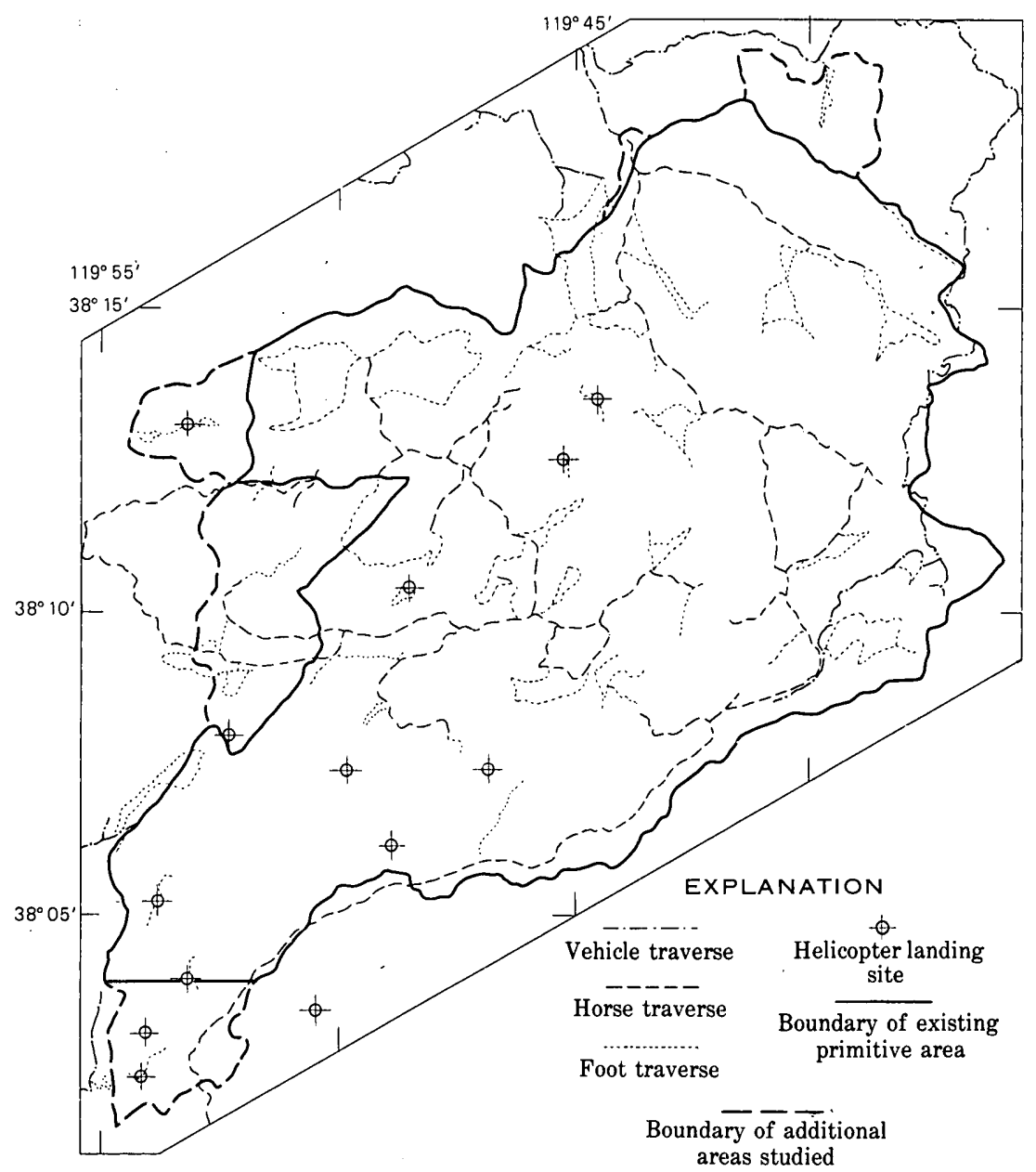

FIgURE 3.-Geologic traverses in the Emigrant Basin primitive area, California.

reached by helicopter. This means of transportation was excellent for low-level observations of the general geology of the area. Finally, traverses by jeep were made along all logging and mine roads and trails in the adjoining areas, especially along the roads leading to the inactive uranium mines and prospects that lie northwest of the primitive area.

The reconnaissance geologic map of the Emigrant Basin primitive area, at a scale of $1: 62,500$ (pl. 1), shows that the metamorphosed sedimentary rocks are preserved as roof pendants and septa, the granitic rocks intruded these metasedimentary rocks, and the volcanic rocks and unconsolidated surficial deposits overlie the older rock units. Knowledge of the distribution and structural characteristics of the main rock types was used in planning the geochemical sampling. No 
new subsurface geologic exploration, such as drilling, was done, although all existing mine openings were carefully examined.

The geochemical study of the Emigrant Basin area consisted chiefly of sampling and chemically analyzing stream sediments; however, representative samples of unaltered country rock, visibly altered rocks, veins, breccia zones, alluvium, and soil were also analyzed. Nearly 400 samples were collected along about 230 miles of traverses. The distribution of these samples is shown in figure 4 . The samples were analyzed

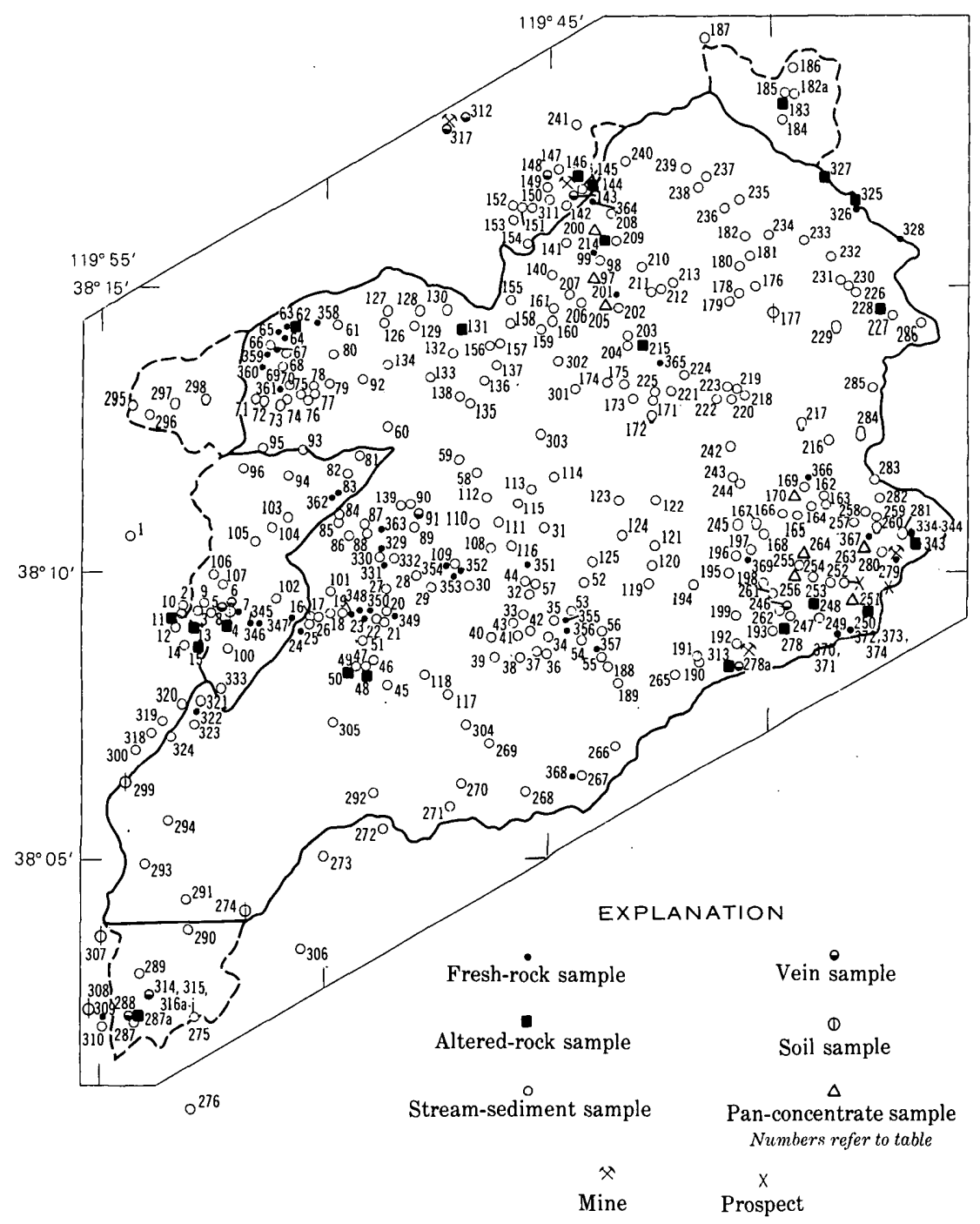

Figure 4.-Location and type of analyzed samples, Emigrant Basin primitive area, California. Symbols indicate sample types; letters are used in text and table. 
by spectrographic, atomic absorption, wet chemical, or colorometric methods. A portable Geiger counter and a shortwave ultraviolet lamp were also used in the field to help detect radioactive uranium and fluorescent tungsten minerals.

The principal geophysical study of the area consisted of an aeromagnetic survey that was made in March 1968. The magnetic data were contoured and are shown on the geologic map, plate 1. An evaluation of the aeromagnetic studies by $\mathrm{H}$. W. Oliver is presented on pages G44-G49.

\section{GEOLOGIC SETTING}

The Emigrant Basin primitive area is in the west central part of the Sierra Nevada, a range of mountains that trends north and is about 40 to 80 miles wide and 400 miles long. The range is asymmetrical, with a gently inclined western slope and a steep escarpmentlike eastern slope that resulted from the westward tilting of an immense structural block. This block consists chiefly of a batholith of granitic rocks and roof pendants and septa of metasedimentary rocks. ${ }^{1}$ In the central Sierra, these rocks are discontinuously overlapped on the eastern side by a partly eroded blanket of Tertiary volcanic rocks, and on the western side they are overlapped by both volcanic and sedimentary rocks that are also of Tertiary age.

As described by Bateman and Wahrhaftig (1966, p. 107), five major geologic events are recorded in the rocks of the central Sierra Nevada: (1) a long period of deposition of marine sediments during the Paleozoic Era (from 230 to 600 m.y. (million years) ago), (2) formation of a great synclinorial basin during latest Paleozoic or earliest Mesozoic time that was subsequently filled by volcanic and sedimentary debris, deformed, repeatedly intruded by granitic batholithic masses, and finally deeply eroded before the close of the Mesozoic Era (about 62 m.y. ago), (3) structural stability during the early Cenozoic, (4) uplift, tilting, and faulting, preceded and accompanied by volcanism in the late Cenozoic (9 to 26 m.y. ago), and (5) carving of the present topography by erosion and Quaternary glaciation (about 1 m.y. ago).

\footnotetext{
${ }^{1}$ As used in this report, a "batholith" is a large body of igneous rock that is composed of many separate, coalescing smaller bodies of igneous rock, called plutons; "plutons" generally are less than 40 square miles in surface area, and have steeply dipping contacts with the rocks they intrude; a "pendant" is a wallrock remnant that projects downward into the upper part of a batholith; a "septum" is a wallrock remnant that forms a partition between adjacent plutons in a batholith. Commonly, pendants have greater size and areal continuity than septa, which typically form linear zones of large and small fragments of metamorphic rocks.
} 


\section{ROCK UNITS}

\section{METAMORPHIC ROCKS}

Metamorphosed strata of probable Paleozoic age underlie about 10 percent of the area of exposed rocks. They form a small roof pendant and a partly preserved septum in the batholithic rocks in the southeastern and northwestern parts of the primitive area (pl. 1). The largest remnant of these metamorphic rocks lies along the Yosemite National Park boundary and forms the Bigelow Peak pendant. This pendant consists of tightly folded, locally granulated, medium-grained, plagioclase-pyroxene hornfels, marble, quartzite, tactite, biotite and andalusite schist, and quartz-mica schists. The tactite consists of epidote, garnet, and other aluminum and iron silicate minerals and represents metamorphosed siliceous and argillaceous limestone and dolomite. It occurs in bands and zones near the contacts of the granitic and metasedimentary rocks in the pendant and is the host rock for deposits of tungsten and other ores. Dark-colored irregular bodies of hornblendite and gabbro, and light-colored dikes of aplite and pegmatite intrude these rocks but are not shown on plate 1. Although the metamorphosed strata in the Bigelow Peak pendant are complexly folded, the beds generally strike northwest and dip steeply to the northeast and southwest. Because of the intensity of the compressive force, some of the more easily deformed units, such as marble, were stretched and segmented into discrete lenses (fig. 5) that can be traced for only short distances.

A second smaller less well defined and discontinuous zone of metamorphic rocks, the Toms Canyon septum, extends sinuously from Coopers Meadow through Toms Canyon southwestward into the Bourland Meadow area. The northwest-trending steeply dipping schists, hornfels, and quartzite of this septum are intruded by granitic rocks at Leopold Lake (fig. 6) and are also cut by an array of aplite and diorite dikes. Rocks of the septum, however, do not include the altered iron-stained marble and tactite zones that are characteristic of the Bigelow Peak pendant.

In general character and lithology, metamorphic rocks of the pendant and septum resemble the late Paleozoic Calaveras Formation exposed near Sonora, about 30 miles west of the Emigrant Basin area.

\section{IGNEOUS ROCKS}

Intrusive and extrusive igneous rocks underlie about 90 percent of the Emigrant Basin primitive area. Pre-Cenozoic batholithic rocks, which consist of large plutonic bodies of granodiorite, quartz-monzonite, granite, and alaskite, intrude folded metamorphosed strata and 


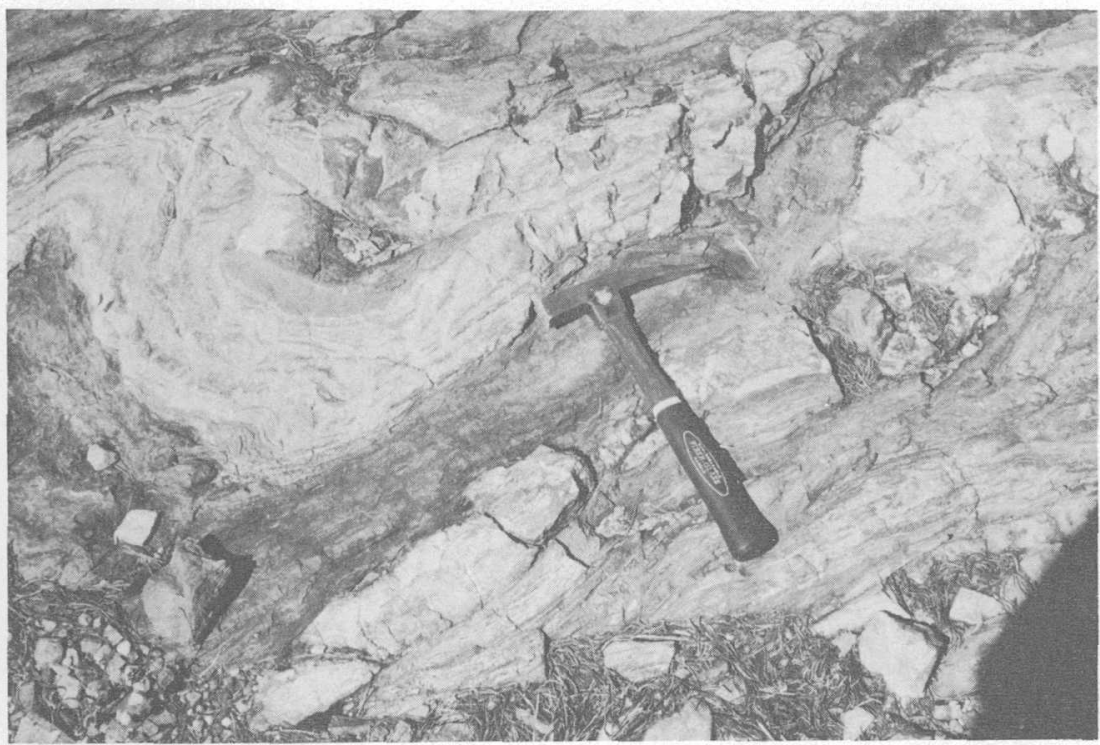

EIgure 5.-Typical tightly folded epidote-garnet tactite lens in metasedimentary rocks, Bigelow Peak roof pendant near Sachse Monument.

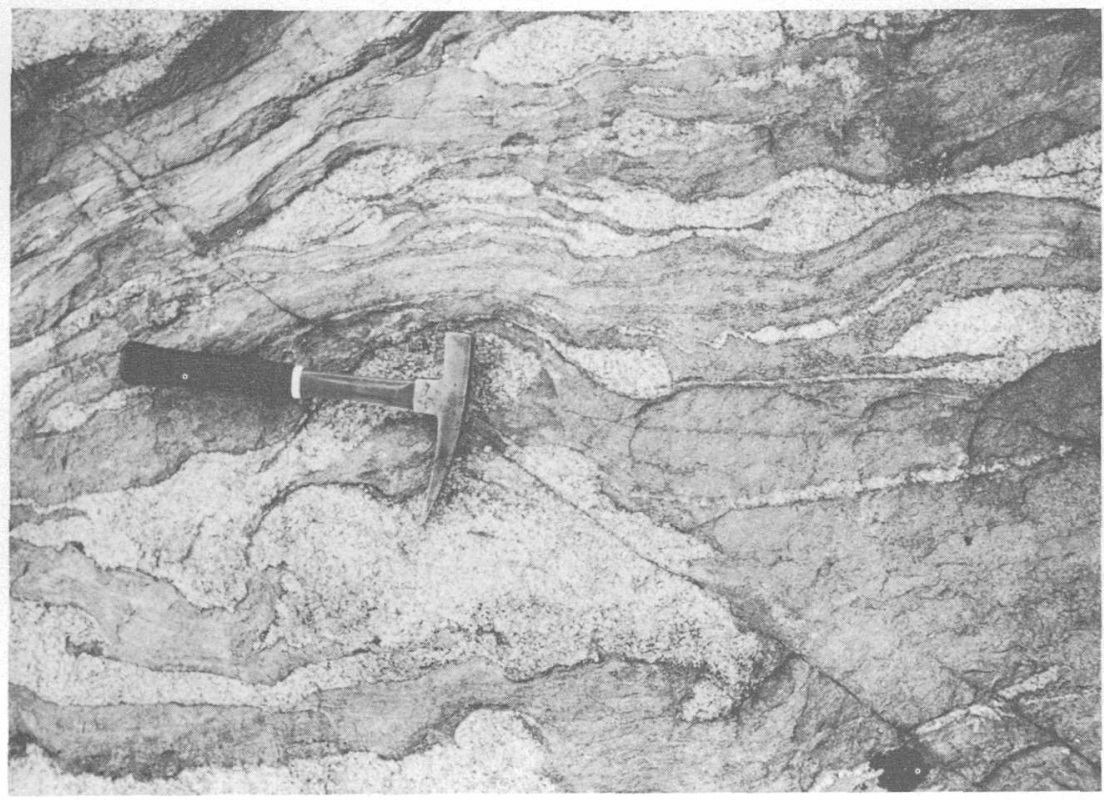

FIGURE 6.-Steeply dipping metasedimentary gneissic rock (dark gray) intruded along bedding by granitic rocks (light gray) in the Toms Canyon septum near Leopold Lake. 
are in turn intruded by later intrusive plugs, dikes, and sills of diorite, hornblendite, gabbro, aplite, and pegmatite. Late Tertiary latite, andesite, rhyolite, and basalt flows and pyroclastic deposits, which covered a deeply eroded terrain are preserved over most of the northern part of the primitive area. Small andesitic intrusive bodies, similar in age and composition to the volcanic rocks, crop out in the vicinity of Relief Reservoir.

\section{BATHOLITHIC GRANITIC ROCKS}

Although the granitic rocks are undifferentiated on plate 1, they actually consist of parts of four or more individually intruded, petrographically and structurally distinct, plutons (D. B. Slemmons, written commun., 1965, and Clyde Wahrhaftig, oral commun., 1968). Chemical analyses of representative samples of these plutons were made by James Sjoberg (written commun., 1966) for the NASA test site program at the University of Nevada and are presented in table 1. In general, the plutons are sequentially younger from west to east. This is also the general order of intrusion in the Yosemite National Park area as was indicated by Calkins (1930) and was subsequently confirmed by geochronologic studies by Bateman, Clark, Huber, Moore, and Rinehart (1963). The individual plutons are distinctively textured and can readily be distinguished, although some of the boundaries are not sharply defined. All of the granitic rocks are conspicuously jointed or sheeted, and planar structures defined by oriented xenolithic inclusions and platy minerals are conspicuous near pluton margins.

The granitic rocks described by D. B. Slemmons (written commun., 1966) in the central part of the area east of the Toms Canyon septum are considered to be part of the quartz monzonite of Patterson Grade. These rocks are medium-grained granular quartz monzonite, containing 5 to 30 percent quartz, 5 to 15 percent $\mathrm{K}$-feldspar, 50 to 80 percent plagioclase $\left(\mathrm{An}_{30-40}\right), 1$ to 20 percent hornblende, and 2 to 10 percent biotite and accessory minerals. Locally this pluton is porphyritic and contains large well-formed feldspar crystals embedded in a granular groundmass. Similar rocks are also exposed outside of the Emigrant Basin area along State Highway 108 between Niagara Creek and Clark Fork Junction.

The quartz monzonite of Patterson Grade merges eastward into the younger quartz monzonite of Topaz Lake. The Topaz Lake pluton, as exposed near Relief Reservoir, is even more coarsely porphyritic than the Patterson Grade pluton and is characterized by fist-sized phenocrysts of $\mathrm{K}$-feldspar and also, by numerous aplite and pegmatite dikes that are localized by prominent joint systems. This pluton resembles the Cathedral Peak Granite described by Calkins (1930) in Yosemite 
TABLE 1.-Chemical analyses of granitic rock units in Emigrant Basin primitive area

[Analyses by Nevada Mining Analytical Laboratory, Reno, Nev.]

\begin{tabular}{|c|c|c|c|c|c|}
\hline Oxides & $\begin{array}{l}\text { Granodiorite of } \\
\text { Cascade Creek }\end{array}$ & $\begin{array}{l}\text { Granodiorite of } \\
\text { Patterson Grade }\end{array}$ & $\begin{array}{l}\text { C. } \\
\text { Quartz monzonite } \\
\text { of Topaz Lake }\end{array}$ & $\begin{array}{l}\text { Alaskite granite } \\
\text { of Dorothy Lake }\end{array}$ & $\begin{array}{l}E \text {. } \\
\text { Quartz monzonite } \\
\text { of Bond Pass }\end{array}$ \\
\hline $\mathrm{SiO}_{2}$ & 63.5 & 63.2 & 67.7 & 74.0 & 66.4 \\
\hline $\mathrm{Al}_{2} \mathrm{O}_{3}$ & 15.4 & 15.7 & 15.6 & 14.9 & 16.4 \\
\hline${ }^{1} \mathrm{Fe}_{2} \mathrm{O}_{3}$ & 1.7 & 1.6 & .9 & .7 & 1.0 \\
\hline $\mathrm{FeO}$ & 3.4 & 3.2 & 1.8 & 1.4 & 2.0 \\
\hline $\mathrm{MgO}$ & 2.0 & 2.0 & .9 & .3 & .9 \\
\hline $\mathrm{CaO}$ & 4.2 & 4.2 & 2.6 & .9 & 2.8 \\
\hline $\mathrm{Na}_{2} \mathrm{O}$ & 3.5 & 3.6 & 3.9 & 4.5 & 4.4 \\
\hline $\mathrm{K}_{2} \mathrm{O}$ & 2.7 & 3.1 & 4.0 & 4.8 & 3.4 \\
\hline $\mathrm{H}_{2} \mathrm{O}$ & .1 & .1 & .1 & .1 & .1 \\
\hline $\mathrm{TiO}_{2}$ & .7 & .8 & .5 & .3 & .6 \\
\hline$P_{2} O_{5}$ & .2 & .2 & .2 & .04 & .1 \\
\hline $\mathrm{MnO}$ & .1 & .1 & .1 & .1 & .1 \\
\hline Total & 97.5 & 97.8 & 98.3 & 102.0 & 98.2 \\
\hline
\end{tabular}

$$
2.010 \text {. }
$$

${ }^{\mathrm{FeO}} / \mathrm{Fe}_{2} \mathrm{O}_{3}$ ratio is assumed to be 2.000 except for the quartz monzonite of Bond Pass, where it is

Location of analyzed samples:

A. Roadcut along Highway 108 near Cascade Creek, NWI/4, NWI/4 sec. 23, T. 5 N., R. 18 E. Dardanelles Cone quadrangle.

B. Roadcut on Highway 108 west of Donnells Reservoir center SEI/4 sec. 30, T. 6 N., R. $19 \mathrm{E}$. Dardanelles Cone quadrangle.

. Roadcut on Highway 108 near Blue Canyon-Deadman Creek junction, Sonora Pass quadrangle.

Didge crest north of Dorothy Lake Pass on boundary Yosemite National Park, T, 4 N., R, 22 E. Tower Peak quadrangle.

E. Ridge crest at Bond Pass, T. 4 N., R. 21 E., Tower Peak quadrangle.

Valley. According to D. B. Slemmons (written commun., 1966), the quartz monzonite of Topaz Lake contains 20 to 30 percent quartz, 10 to 20 percent $\mathrm{K}$-feldspar, 40 to 50 percent plagioclase $\left(\mathrm{An}_{25}\right)$, and 5 to 10 percent mafic and accessory minerals.

Textural contrasts noted across plutonic contacts are best demonstrated along the North Fork of Cherry Creek northeast of Emigrant Lake, between the light-colored porphyritic quartz monzonite of the Topaz Lake pluton, which adjoins the darker gray equigranular granodiorite of the Tilden Lake pluton. Near the contact zone the granodiorite contains many biotite-rich dark-gray xenolithic inclusions, which gradually diminish in number and size away from the contact. The Tilden Lake pluton, named for exposures near Tilden Lake, 3 miles southeast of Bigelow Peak, is in contact with the Bigelow Peak roof pendant in the southeastern part of the area. Smaller distinct intrusive bodies, which also adjoin the roof pendant and the Tilden Lake pluton along the southeastern border of the area, include (1) the Dorothy Lake pluton, a medium- to fine-grained pinkish alaskitic granite containing a minor amount of biotite, and (2) the Bond Pass pluton, a medium-grained, light-gray quartz monzonite containing quartz, $\mathrm{K}$-feldspar, plagioclase, and biotite. Immediately outside the southeast margin of the primitive area near Forsyth Peak is yet another pluton, a granodiorite, mapped by F. E. Matthes in 1937 and in greater detail by Clyde Wahrhaftig (oral commun., 1968). Along 
the southern boundary of the Emigrant Basin primitive area, southwest of Bigelow Peak, the granitic rocks are largely part of the El Capitan Granite, described in the Yosemite Valley by Calkins (1930).

West of the Toms Canyon septum and adjoining the Patterson Grade pluton are several other plutons including the Cascade Creek pluton, a granodiorite, and the Mill Creek pluton, a quartz monzonite (D. B. Slemmons, written commun., 1966). In general these are finer grained, less porphyritic rocks containing 20 percent quartz, 40 percent K-feldspar, 30 percent plagioclase, 5 percent biotite, and 1 percent hornblende. Near their contacts with the dioritic and metasedimentary rocks, the borders of many of these plutons characteristically contain swarms of dark-colored xenoliths a few inches to tens of feet in length and breadth. These are locally cut by aplite dikes (fig. 7).

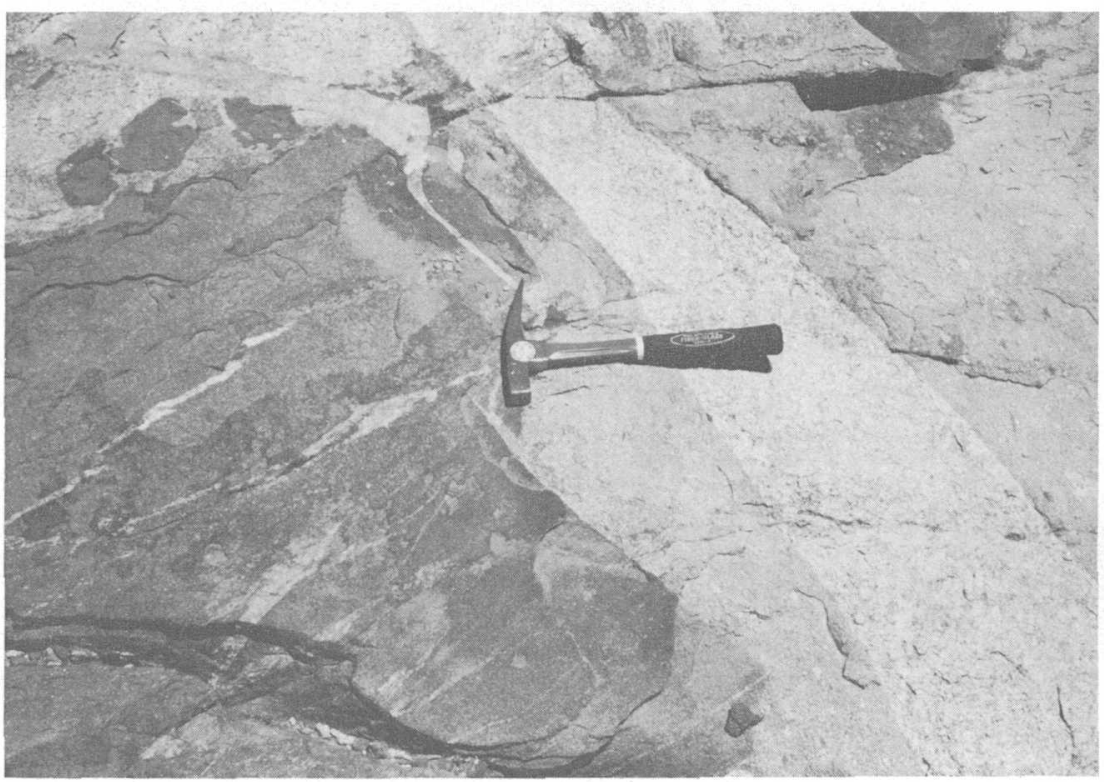

FIGURE 7.-Dioritic xenolith (dark gray) in porphyritic granitic rock (gray) cut by an aplite dike (light gray) near the junction of Buck Creek and the West Fork of Cherry Creek in Louse Canyon.

\section{DIKE ROCKS AND PLUGS}

The smaller intrusive bodies exposed in the Emigrant Basin area generally fall into one of two age groups. The older group includes dikes and small plugs that range in composition from aplite to gabbro and range in size from a few inches to nearly 100 feet in width and from tens of feet to as much as half a mile in length. They are most conspicuous where they cut the roof pendant and the granitic rocks 
near the margins of plutons, but they are common throughout all the granitic rocks. Most of these small bodies have not been differentiated on plate 1 ; however, some of the more conspicuous diorite dikes associated with the Toms Canyon septum are shown. Diorite, in the general field sense, is a medium- to coarse-grained igneous rock that is low in quartz but contains abundant plagioclase feldspar and mafic minerals, such as amphibole and biotite. Other dikes that are especially numerous near the pendants and septae are composed primarily of feldspar, mica, and quartz with only minor amounts of other minerals; the fine-grained granular dikes are called aplite, and the coarse-grained ones are called pegmatite. A few of the pegmatite dikes that cut the pendant rocks also contain coarse-textured tourmaline in addition to quartz, feldspar, and mica. Other intrusive bodies of the older group include small, predominantly brown weathering, medium-grained, pluglike and dikelike masses of pyroxene- and plagioclase-rich hornblendite and gabbro that intrude the metasediments and granitic rocks of the Bigelow Peak roof pendant. These bodies largely account for the dark color of the ridge separating the primitive area and Yosemite National Park; they were mapped by Clyde Wahrhaftig (written commun., 1968).

The younger group of dikes, sills, and intrusive plugs consists of dark-brown, fine-grained, hornblende andesite and latite. These intrusives cut the volcanic rocks and all of the older granitic and metamorphic rocks. They are similar in texture and composition to the volcanic rocks and are presumed to be the source of some of the younger flows and pyroclastic deposits.

A semiconsolidated prevolcanic arkosic sand and grit derived from the weathering of prevolcanic granite, presumably during Miocene time, crops out on an exhumed surface above Snow Lake and underlies the volcanic sequence on Grizzly Peak. Erosion produces very distinctive unconsolidated debris.

\section{VOLCANIC ROCKS}

During the latter half of the Tertiary Period, after the metamorphic and intrusive rocks had been deeply eroded and the core of the batholith had been exposed, great masses of volcanic rocks were erupted over the Emigrant Basin area. The areal distribution, lithology, and age of these eruptive rocks have been described by Slemmons (1966) and the geologic history of the volcanic episode has been reviewed by Bateman and Wahrhaftig (1966). Deep erosion has largely stripped the volcanic rocks from the eastern and southern areas. These rocks are now found chiefly in the northern third of the primitive area and along the western border. (See sections, pl. 1.) Table 2 is a schematic representation of the stratigraphic relations of the volcanic formations. 
The volcanic rocks range in composition from rhyolite to basalt but consist mostly of latite and andesite tuffs, agglomerates, autobrecciated flows, and a few massive flows. They were erupted chiefly from volcanic centers some distance east of the Emigrant Basin area. West of the primitive area, they interfinger with clastic sedimentary deposits composed largely of volcanic debris.

The oldest unit is the Valley Springs Formation of Slemmons (1966), of early and middle Miocene age. It consists of a series of rhyolitic and dacitic welded tuffs that were widely distributed in the northern Sierras. Locally, they were as much as 1,200 feet thick.

The most prevalent volcanic rocks in the primitive area are the agglomerates of the Relief Peak Formation of Slemmons (1966), the type section of which is in secs. 18, 19, and 20, T. 5 N., R. 21 E., in the northwestern part of the proposed wilderness area (Slemmons, 1966, p. 203). These agglomerates, or mudflow breccias, consist of poorly sorted, moderately rounded pebbles, cobbles, and boulders of andesite

TABLE 2.-Volcanic rocks in the Emigrant Basin primitive area

[Age and names of rock units are from Slemmons (1966)]

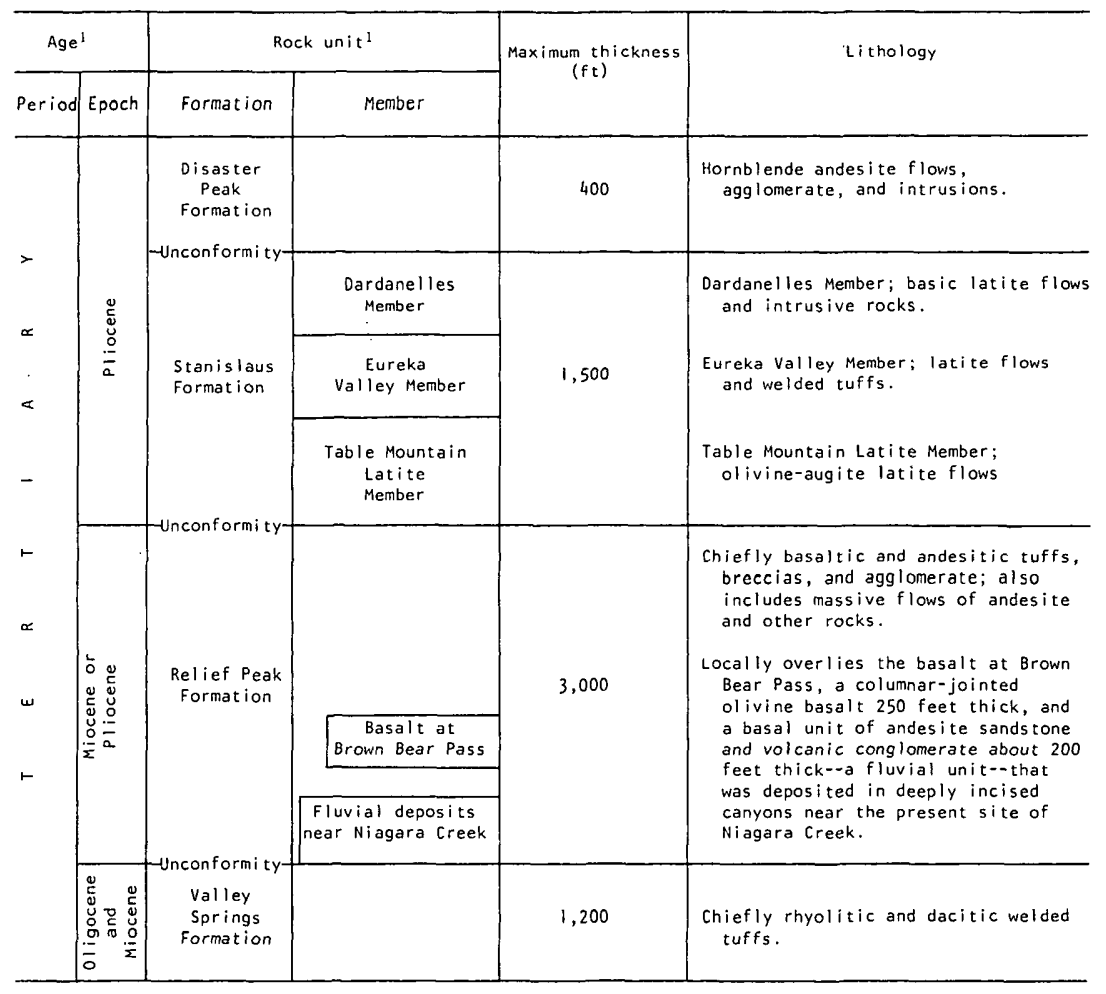

'Age and names of rock units are from slemmons (1966). 
that are embedded heterogeneously in a matrix of volcanic tuff and arkosic sandstone. Locally, the agglomerates contain layers of massive and autobrecciated andesite flow rocks and lenses of volcanic sandstone and conglomerate.

In the Relief Peak area, the volcanic rocks of the Relief Peak Formation covered an erosion surface of pronounced relief and filled ancient valleys that were eroded into both the Valley Springs Formation and the pre-Cenozoic, granitic and metamorphic basement rocks (fig. 8). These dendritic valley systems generally extend downslope, and they broaden to the southwest (Slemmons, 1966, p. 203). In many of the prelava drainage courses the andesitic rocks have buried fluvial sand and gravel deposits, considered by Slemmons to be the lowest unit within his Relief Peak Formation, that are composed in part of debris from both the underlying rhyolitic rocks of the Valley Springs Formation and the granitic and metamorphic basement rocks. D. B. Slemmons (oral commun., 1967) described these stream channel deposits at Niagara Creek, adjacent to the northwestern part of the primitive area. Near Brown Bear Pass, on the higher parts of one of the pre-andesite valleys, the agglomerates and flow rocks of the Relief Peak Formation directly overlie the basalt at Brown Bear Pass. However, throughout the higher parts of the primitive area,

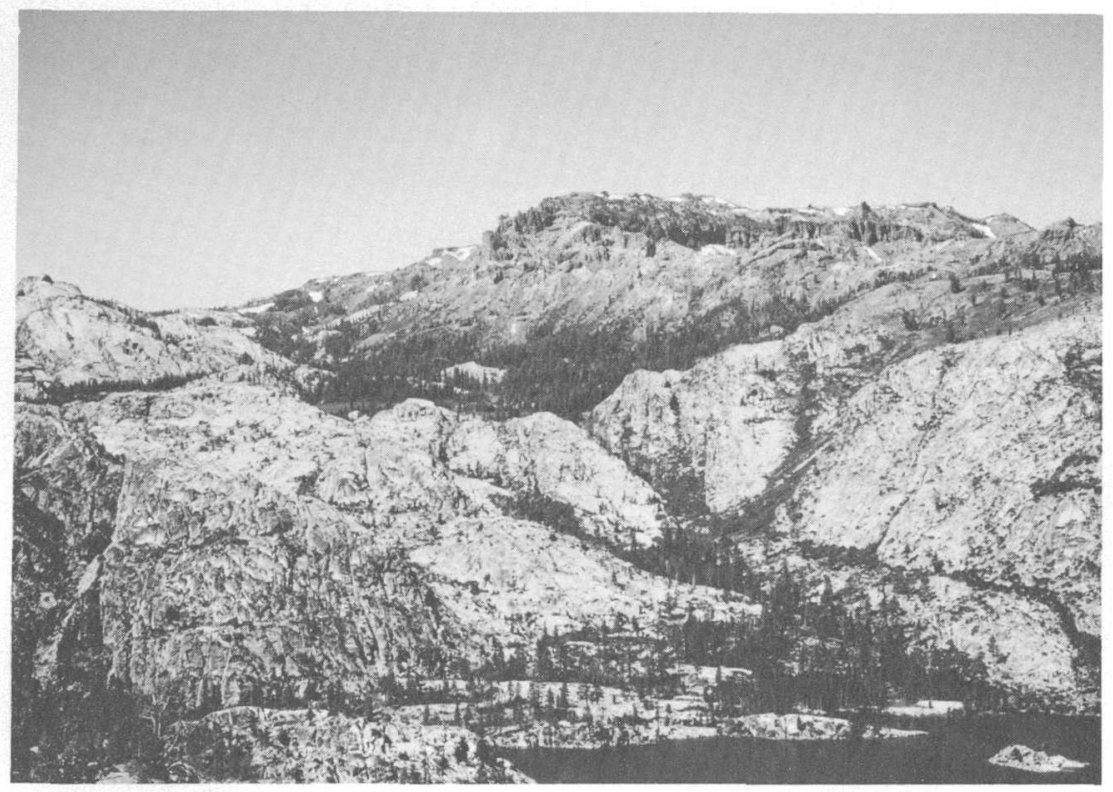

Figure 8.-East Flange Rock on skyline consists of volcanic rocks (dark gray) filling a prelava erosion valley cut in granite (light gray). The head of Relief Reservoir is in the foreground. 
the Relief Peak Formation generally overlies pre-Cenozoic granitic and metamorphic rocks and only a few scattered remnants of the Valley Springs Formation.

At the northern boundary of the primitive area between Night Cap Peak and peaks on the Sierra crest southwest of Leavitt Lake, Slemmons recognized that the Relief Peak Formation was unconformably overlain by remnants of the Table Mountain Latite Member, the basal member of his Stanislaus Formation. Elsewhere in the primitive area, post-Relief Peak volcanic units are absent or were not observed.

Chemical compositions of samples of rhyolite from the Valley Springs Formation and basalt from Brown Bear Pass have been determined by James Sjoberg (written commun., 1966) and are presented in table 3.

Slemmons $(1966$, p. 199, 203) considered the Relief Peak Formation to be of late Miocene or early Pliocene age on the basis of potassiumargon dates from minerals in the rocks and fossil flora dates from interbedded fluvial deposits. The profound nature of the unconformity separating the Valley Springs and Relief Peak Formations serves as supporting evidence for the apparent difference in their ages. The potassium-argon age of the Stanislaus Formation, which unconformably overlies the Relief Peak Formation, ranges from 8.8 to 9.3 million years, indicating an early Pliocene age. The relatively minor nature of the unconformity separating the Relief Peak Formation and the Stanislaus Formation also provides supporting evidence for a late Miocene or early Pliocene age for the Relief Peak Formation.

TABLE 3.-Chemical analyses of two volcanic rocks, Emigrant Basin primitive area

[Analyses by Nevada Mining Analytical Laboratory, Reno, Nev.]

\begin{tabular}{|c|c|c|}
\hline Oxide & $\begin{array}{l}\text { Valley Springs } \\
\text { Formation of } \\
\text { Slemmons } \\
(1966) 1\end{array}$ & $\begin{array}{c}\text { Basalt at } \\
\text { Brown Bear } \\
\text { Pass }^{2}\end{array}$ \\
\hline $\begin{array}{l}\mathrm{SiO}_{2} \\
\mathrm{Al}_{2} \mathrm{O}_{3} \\
\mathrm{Fe}_{2} \mathrm{O}_{3} \\
\mathrm{FeO}^{\mathrm{MgO}} \\
\mathrm{MaO} \\
\mathrm{Na}_{2} \mathrm{O}_{2} \\
\mathrm{~K}_{2} \mathrm{O} \\
\mathrm{H}_{2} \mathrm{O} \\
\mathrm{TiO}_{2} \\
\mathrm{P}_{2} \mathrm{O}_{5} \\
\mathrm{MnO}\end{array}$ & $\begin{array}{r}\text { 76. } 6 \\
11.4 \\
.2 \\
.5 \\
.1 \\
.5 \\
2.6 \\
5.3 \\
.04 \\
.2 \\
.01 \\
.1\end{array}$ & $\begin{array}{r}51.8 \\
15.9 \\
2.2 \\
8.6 \\
7.2 \\
6.7 \\
3.2 \\
1.9 \\
.5 \\
1.9 \\
.6 \\
.2\end{array}$ \\
\hline Total & 97.5 & 100.7 \\
\hline
\end{tabular}

1 Exposures near Grizzly Peak, T. 4 N., R. 21 E., Tower Peak quadrangle.

2 Exposures near Brown Bear Pass, 1 mile northwest of Emigrant Meadow Lake, T. 4 N., R. 21 E., Tower Peak quadrangle. 


\section{UNCONSOLIDATED DEPOSITS}

The thorough glacial scouring of the Emigrant Basin area that took place during the Pleistocene age removed much of the unconsolidated surficial debris and left extensive areas of bare rock exposed (fig. 9). The unconsolidated deposits that remain, or have accumulated since the glaciations, include moraines, alluvium, talus, and bog deposits.

In areas underlain by granitic and metamorphic rocks, the principal unconsolidated deposits consist of sand- and gravel-sized alluvium and humic silt. These deposits usually fill shallow rock-floored basins formerly occupied by lakes. This creates the picturesque meadows that contribute so greatly to the beauty of the primitive area. Similar accumulations of alluvium are also found here and there in some stream valleys and gulches, particularly where rock slides and moraines have formed small dams.

In volcanic terrane, the principal unconsolidated deposits are extensive sheets and aprons of talus that have been derived from the glacially oversteepened slopes on the easily eroded agglomerate of the Relief Peak Formation of Slemmons (1966) (fig. 10).

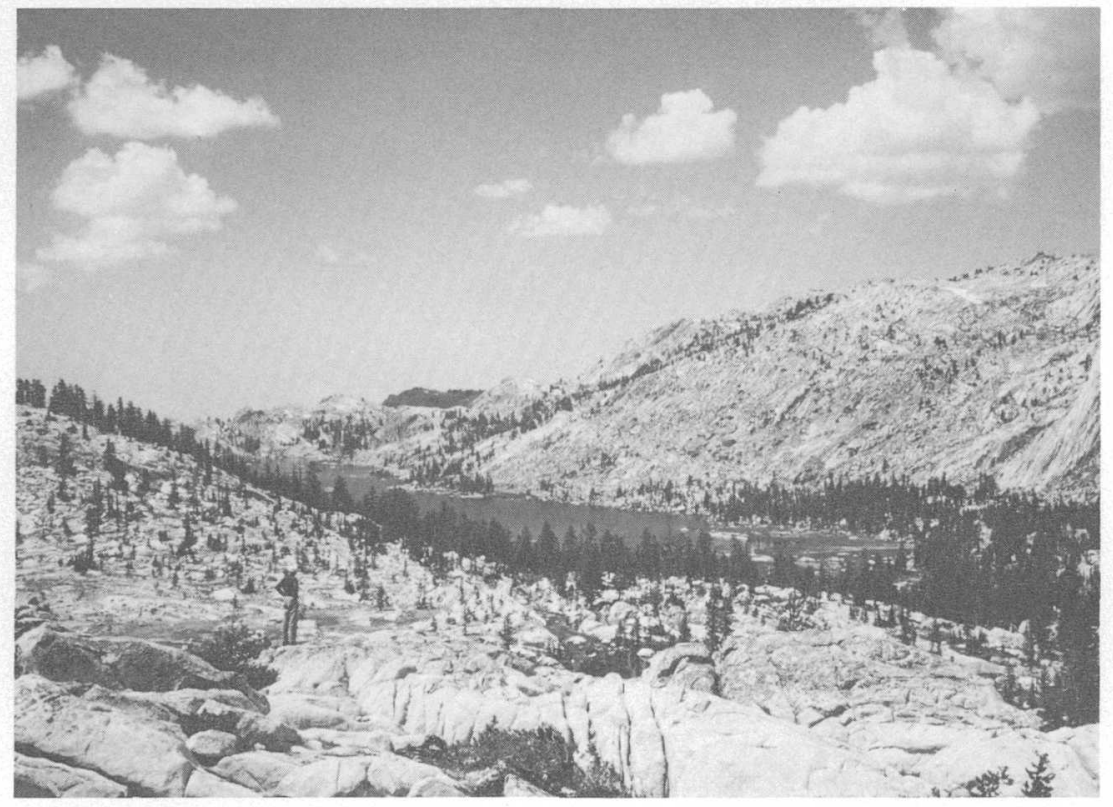

Figure 9.-Typical glacial-scoured area about Emigrant Lake and small postglacial meadow formed at the lake inlet. 


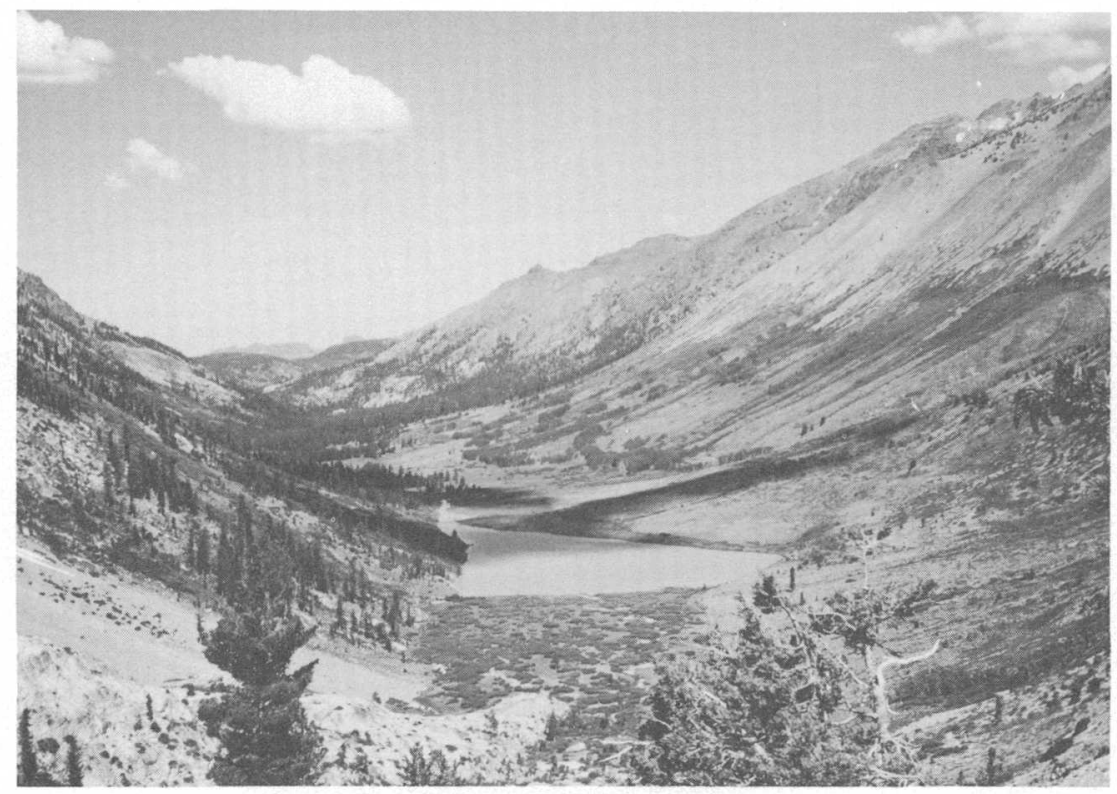

Figure 10.-U-shaped Kennedy Valley floored by granite with volcanic rock walls. Kennedy Lake is in foreground.

The glacial deposits of the Emigrant Basin area consist of a few end moraines and some patchy deposits of lateral moraine. The lack of soil on these moraines suggests that they are products of the Tioga Glaciation of late Pleistocene age, or, of the more recent Little Ice Age of 2,000 to 2,700 years ago. In general, glacial deposits are less abundant in the Emigrant Basin area than they are at lower elevations where the glaciers terminated.

In addition to these more common types of surficial deposits, a rather unique deposit of unconsolidated material occurs near the crest of the Sierra Nevada near Grizzly Peak. It consists largely of arkosic sand and grit derived from semiconsolidated prevolcanic weathered granitic rock, presumably of Miocene age, that has been exposed by the removal of overlying lava and agglomerate. Erosion of this material during the present climatic cycle has gullied it deeply and redeposited a large quantity of the decomposed granitic material over broad areas in the upper reaches of Emigrant Meadows.

\section{GEOLOGIC HISTORY}

The rocks and geologic structures of the central Sierra Nevada record a complex history of deformation. Regional analysis of the structure of the stratified sedimentary rocks of the roof pendants of the Emigrant Basin primitive area and elsewhere in the range 
(Bateman and Eaton, 1967) indicates that, during Late Jurassic time, marine sedimentary rocks of Paleozoic and Mesozoic age were folded into a northwest-trending synclinorium and subsequently faulted on the west side. Steep bedding attitudes and the distribution of xenoliths provide evidence that the syncline extended many miles below present exposures, and the degree of thermal metamorphism of the rocks indicates that those now exposed were once overlain by as much as 9 miles of cover (Bateman and Wahrhaftig, 1966). Continuing deformation during the emplacement of the granitic batholith by the injection of a series of separate plutons, during latest Jurassic and earliest Cretaceous time, is indicated by the shattering and foliation of the rocks near the margins of the intrusive bodies.

After the batholith had consolidated, it was jointed and locally fractured into large sheeted zones. The joints are most conspicuous in the eastern part of the range, where they form a conjugate system of steeply dipping fractures that trend north-northwest and east-northeast. They are spaced from a few feet to many feet apart. The uneven spacing apparently resulted chiefly from differences in the grain size of the granitic rocks. Some individual joints extend for several miles and cross from one pluton into another without change of direction. Locally, some of the more persistent joints or closely spaced groups of joints strongly affect erosion patterns. According to Bateman and Wahrhaftig $(1966$, p. 122), sheeting is more common in the western part of the batholith where jointing is absent, and in this area it facilitates the development of the great exfoliation domes that are such distinctive features of Sierran landscapes.

By Eocene time, erosion had bared the batholith and created a rolling upland surface on the ancestral Sierra Nevada that shed debris westward to form the sedimentary deposits that now overlap the batholithic rocks. Volcanism was widespread during the Miocene and Pliocene Epochs. Unconformities between some of the volcanic units indicate periods of intravolcanic uplift and erosion. During late Pliocene time, after volcanism subsided, the northern Sierras were strongly uplifted and tilted to the west, and the fault systems, which produced the eastern scarps of the range, began to develop. Later, during the Pleistocene, several glaciations and subsequent subaerial erosion (Wahrhaftig, 1962) of this terrain produced the picturesque landscape of the primitive area. The sheeted granitic rocks were plucked and smoothed and this produced boulder-strewn slopes or low rounded domes and ridges. Joint-oriented streams connect rockbound lakes such as Emigrant Lake (fig. 9) and Shallow Lake (fig. 2). In contrast, glaciation of volcanic rocks produced long U-shaped valleys (fig. 10) that are floored in granite and head in deep steep-walled cirques that commonly contain lakes fed by one or more cascading waterfalls. 


\section{ORE DEPOSITS IN THE REGION}

The Sierra Nevada has been a major source of mineral commodities since gold was discovered in California in 1848 (Clark, 1966, p. 209). Most of these mineral deposits are peripheral to the great batholith that forms the core of the range and are found chiefly in the sedimentary and metamorphic wallrocks, in surficial deposits of the westem foothills, and in volcanic rocks along the faulted eastern margin of the range. A smaller number of deposits occur in the metamorphic roof pendant rocks in the batholith, but virtually no deposits in the granitic core rocks and in the volcanic rocks in the central part of the range have proven to be of commercial value.

The best known and most productive ore deposits in the general region are the gold deposits of the Mother Lode belt in the Sierra foothills 30 to 50 miles west of the Emigrant Basin primitive area. The primary gold deposits of the Mother Lode are gold-quartz veins in slate, schist, and greenstones that were invaded by small granitic plutons (Clark, 1966, p. 209). The veins range from a fraction of an inch to tens of feet in thickness; some consist of parallel and branching quartz stringers separated by slabs of country rock. The gold occurs as visible to microscopic grains, plates, and threads in the quartz and is accompanied by pyrite, arsenopyrite, and small amounts of other sulfides. Many of the ore shoots extend to depths of only a few hundred feet; others, to depths of several thousand feet.

Of somewhat greater value in terms of gross production, were the placer deposits associated with the gold-bearing veins in the foothills to the west of the primitive area. These deposits were largely exhausted by 1880 , but they originally consisted of modern and ancient stream gravels locally containing rich pay streaks of native gold associated with detrital magnetite, ilmenite, zircon, garnet, and other heavy dark minerals. The modern, or Holocene, placers were found at one or more levels in the present stream channels; the ancient placers were of Tertiary age and consisted of alluvial deposits that were preserved beneath thick flows of volcanic rocks. Some of these Tertiary deposits were mined by underground methods in workings that followed the twisting courses of the prelava stream beds.

Currently productive mines in the Sierra Nevada include the famous Pine Creek and Strawberry tungsten deposits (Bateman, 1965 ; and Krauskopf, 1953). Both of these are located south of Yosemite National Park in mineralized roof pendants comparable in some respects to the roof pendant rocks in the southern part of the Emigrant Basin area. The Pine Creek mine is the leading tungsten producer in the United States, and, according to Bateman (1965), it is located in the largest contact-metamorphic ore deposits in the world. 
Several other tungsten deposits of a similar type occur 10 miles west of the primitive area, near Confidence, Calif. Here irregular "veins" (mineralized tactite zones) were formerly mined from pendants and along the contact of intrusive granodiorite bodies with the Calaveras(s) Series of Little (1942). These deposits are spatially related to the so-called East Belt deposits of the Mother Lode system of gold-quartz veins.

Lode gold in altered andesite as well as tungsten and base metal deposits in contact metamorphosed rock also occur in the intermittently active Monitor and Silver King mining district (Junkins and others, 1949, p. 33) in Alpine County, 10 to 20 miles north and northeast of the Emigrant Basin area. Other base- and precious-metal mining districts are located east of the Emigrant Basin primitive area in western Nevada and the adjacent part of California. Perhaps the most famous is the Bodie district, about 30 miles due east of the Emigrant Basin primitive area (McLaughlin, 1907). Uranium deposits occur close to the primitive area in ancient stream channels in the fluvial deposits near Niagara Creek. These deposits are more fully described on pages G49-G50.

\section{GEOCHEMICAL STUDIES}

\section{GENERAL OBJECTIVES AND SAMPIING PROCEDURES}

A program of geochemical exploration was undertaken to supplement the geologic studies. Under this program nearly 400 samples of stream sediments, rocks, and minerals were collected, analyzed, and evaluated in terms of their mineral and metal content and their geologic environment.

By far the largest number of samples collected consisted of stream sediments and other alluvial materials that contain the weathered products of eroded rocks. Soluble products from weathered rocks and mineral deposits tend to be adsorbed by the clay fraction of the sediments, whereas insoluble minerals tend to be concentrated by the winnowing action of streams. The typical sediment sample consists of fine- to coarse-grained sand taken from streambeds. Samples were taken particularly from areas of sudden change in the flow gradient such as bars on the inside of meander bends, deltas at lake inlets, eddy deposits in the downstream "shadow" of cobbles and boulders, and accumulations in the bottoms of potholes. A few sampies were further concentrated by panning, and the heavy mineral fractions were submitted for analysis. Because of the extensive amount of time consumed in the panning, and because of the generally disappointing results, only a few of the samples were panned during the mineral survey, and then, only from those areas considered to have a reasonable potential for placer tungsten or gold deposits. 
The rock samples consisted of representative types of all of the fresh unaltered rocks in the area as well as the bleached and ironstained zones along contacts, joints, and fractures that might be the surficial representation of possible concealed ore deposits. A few samples of vein minerals were also collected to test for ore-metal concentrations that could not be detected by visual examination.

The collected samples were dried; pulverized, and then analyzed by spectrographic, atomic absorption, wet chemical, or colorimetric methods. The analyses are presented in table 4, and the sample site locations are shown in figure 4 . Most of the analytical data presented in table 4 were obtained by the six-step semiquantitative spectrographic method and are considered to be within 30 to 50 percent of the correct value. All gold analyses and some copper, lead, and zinc analyses were by atomic absorption methods. Colorimetric methods were used to determine molybdenum and tungsten in some samples, and instrumental detection methods were used on samples tested for mercury. Uranium content was determined by the paper chromatography method. All of these nonspectrographic analytical techniques yield accuracies of 50 to 100 percent. The analytical methods used have been described by Ward, Lakin, and Canney (1963), Huffman, Mensik, and Riley (1967), Vaughn (1967), Thompson, Nakagawa, and Van Sickle (1968), and Grimes and Marranzino (1968).

\section{IIMITATIONS OF THE GEOCHEMICAL DATA}

Geochemical sampling, as used in this study, is not necessarily a complete exploration tool nor is it an infallible guide to areas of metallization. Limitations of both the sample selection and the analytical techniques are well known. For example, (1) not all ore deposits may be identified or located at the surface by means of geochemical anomalies, inasmuch as concealed ore bodies are known to exist beneath undisturbed, unfractured, or impermeable, rock cover, (2) geochemical data often are equivocal unless they are considered in the context of the types of rocks in which they occur and the geologic structures in the area, (3) no systematic attempt was made to select replicate samples, (4) the analytical techniques used are qualitative, and therefore, analyses made from the same sample by two techniques may vary in detail, and (5) finally, and most important, a geochemical anomaly value from a random-grab sample should not be confused with an assay value. However, high values, irrespective of their absolute quantity, indicate the presence of possible ore mineral concentrations. In this context they may be used as guides to undetected deposits, although they do not have validity as either assay or grade 
values. Perhaps of equal importance, it may be assumed that low or average analytical values indicate the virtual absence of mineralized areas.

\section{GEOCHEMISTRY OF ROCKS AND UNCONSOLIDATED DEPOSITS}

\section{Metamorphic ROCKs}

Although most of the metamorphic rocks are unmineralized, anomalous concentrations of tungsten, molybdenum, copper, lead, zinc, manganese, silver, tin, and iron are locally present in parts of the Bigelow Peak roof pendant. However, only a few of these concentrations may be considered to have commercial significance. These elements occur chiefly in limestone or marble layers and lenses that have been metamorphosed to epidote-garnet tactite (samples 341-R, 342-R, table 4) near their contact with intrusive granitic rocks.

Average values for some of these elements in the unmineralized metasedimentary rocks of the Emigrant Basin area are shown in table 5 and are contrasted with worldwide average abundances in sedimentary rocks. As indicated, the metal content of the unmineralized metamorphic rocks of the pendant are generally below the sensitivity limits of the analytical methods used; these rocks are of little interest to the prospector.

The main concentrations of the metallic elements in the limy tactites of the pendant are in the minerals scheelite $\left(\mathrm{CaWO}_{4}\right)$, powellite $\left(\mathrm{Ca}(\mathrm{Mo} \cdot \mathrm{W}) \mathrm{O}_{4}\right)$, molybdenite $\left(\mathrm{MOS}_{2}\right)$, galena $(\mathrm{PbS})$, and pyrite $\left(\mathrm{FeS}_{2}\right)$. Samples 251-C, 278a-V, and $344 \mathrm{~b}-\mathrm{V}$ from mine workings and prospect pits contain as much as $17,000 \mathrm{ppm} \mathrm{Zn} \mathrm{(zinc),} \mathrm{1,500} \mathrm{ppm}$ $\mathrm{Cu}$ (copper), more than $100,000 \mathrm{ppm} \mathrm{W}$ (tungsten), $200 \mathrm{ppm} \mathrm{Pb}$ (lead), 15,000 ppm Mn (manganese), 15 percent Fe (iron), and $5 \mathrm{ppm}$ Ag (silver). Stream-sediment samples taken from near these prospects (samples 256-C, 263-C, 274-S, and 280-S), and apparently unmineralized tactite (samples 248-A and 278-A), also contain anomalous but smaller quantities of these metals.

Argillite, hornfels, and quartzite metasediments (samples 329-R, $336-\mathrm{R}, 337-\mathrm{R}, 340-\mathrm{R}$, and $363-\mathrm{R}$ ), which are interbedded with limestone and are intruded by granite lenses, pegmatite dikes, and dioritic to gabbroic dikes, sills, and plugs (samples 331-R, 334-R, 348-R, and $370-R$ to $374-R$ ) do not contain unusual concentrations of these metals. However, the mafic (dark) intrusive rocks contain slightly greater amounts of nickel, chromium, and magnesium than other rocks in the area, as is typical throughout the world. Sediments from streams draining such rocks generally contain above-average, but nonsignificant, amounts of the same elements.

$368-4560-70-5$ 
TABLE 4.-Analyses of samples from the

[In Sample and type column, type of sample designated by letter following sample numbers: $S=$ stream sediment ; $R=$ bedrock; $C=$ panned concentrate ; $A=$ altered rock; $S o=$ soil ; $V=$ vein minerals. In analyses columns: $G=$ greater than 10 percent, or greater than value shown in "Remarks" column; $L=$ detected, but below limit of determination, or below value shown in parentheses; $N=$ not detected at limit of detection; * =usual limits of determination do not apply, dilution technique used; -- = not looked for. Lower detection limits for analyses are shown either in parentheses at top of columns, or below, in special cases. In "Remarks" column, rock type

\begin{tabular}{|c|c|c|c|c|c|c|c|c|c|c|c|c|c|c|}
\hline \multirow{2}{*}{$\begin{array}{l}\text { Sample } \\
\text { and } \\
\text { type }\end{array}$} & \multicolumn{4}{|c|}{$\begin{array}{c}\text { Semiquantitative spectrographic } \\
\text { analyses (percent) }\end{array}$} & \multicolumn{10}{|c|}{$\begin{array}{c}\text { Semiquantitative spectrographic } \\
\text { analyses }(\mathrm{ppm})\end{array}$} \\
\hline & $\begin{array}{c}\mathrm{Fe} \\
(0.05)\end{array}$ & $\begin{array}{l}\mathrm{Mg} \\
(.005)\end{array}$ & $\begin{array}{c}\mathrm{Ca} \\
(0.05)\end{array}$ & $\begin{array}{c}\mathrm{Ti} \\
(0.001)\end{array}$ & $\underset{(20)}{M n}$ & $\begin{array}{l}\mathrm{Ag} \\
(0.5)\end{array}$ & $\stackrel{B}{(10)}$ & $\begin{array}{l}\mathrm{Ba} \\
(20)\end{array}$ & $\begin{array}{l}\mathrm{Be} \\
(1)\end{array}$ & $\begin{array}{l}\text { Co } \\
(5)\end{array}$ & $\begin{array}{l}\mathrm{Cr} \\
(5)\end{array}$ & $\begin{array}{l}\mathrm{Cu} \\
(2)\end{array}$ & $\begin{array}{l}\text { La } \\
(20)\end{array}$ & $\begin{array}{l}\text { Mo } \\
(5)\end{array}$ \\
\hline $\begin{array}{l}1-S \\
2-S \\
3-S \\
4-A \\
5-V\end{array}$ & $\begin{array}{l}3 \\
1.5 \\
3 \\
15 \\
1.5\end{array}$ & $\begin{array}{l}0.7 \\
.3 \\
.7 \\
3.03\end{array}$ & $\begin{array}{l}1.5 \\
1.5 \\
2 \\
7 \\
.05\end{array}$ & $\begin{array}{r}0.3 \\
.2 \\
.3 \\
G .02 \\
.02\end{array}$ & $\begin{array}{r}300 \\
300 \\
300 \\
1,000 \\
20\end{array}$ & $\begin{array}{l}\mathrm{N} \\
\mathrm{N} \\
\mathrm{N} \\
\mathrm{N} \\
\mathrm{N}\end{array}$ & $\begin{array}{c}L \\
N \\
N \\
10 \\
L\end{array}$ & $\begin{array}{r}1,500 \\
1,500 \\
700 \\
200 \\
30\end{array}$ & $\begin{array}{l}\mathrm{L} \\
\mathrm{L} \\
\mathrm{L} \\
\mathrm{N} \\
\mathrm{L}\end{array}$ & $\begin{array}{r}15 \\
5 \\
15 \\
150 \\
N\end{array}$ & $\begin{array}{r}30 \\
L \\
10 \\
100 \\
L\end{array}$ & $\begin{array}{r}30 \\
15 \\
30 \\
500 \\
10\end{array}$ & $\begin{array}{r}20 \\
20 \\
L \\
L \\
N\end{array}$ & $\begin{array}{l}N \\
N \\
N \\
5 \\
N\end{array}$ \\
\hline $\begin{array}{c}6-V \\
7-R \\
8-5 \\
9-5 \\
10-5\end{array}$ & $\begin{array}{l}3 \\
10 \\
3 \\
1.5 \\
2\end{array}$ & $\begin{array}{l}.3 \\
.7 \\
.2 \\
.5\end{array}$ & $\begin{array}{l}5^{.7} \\
2 \\
1 \\
2\end{array}$ & $\begin{array}{l}G^{.3} \\
.3 \\
.15 \\
.2\end{array}$ & $\begin{array}{l}300 \\
500 \\
300 \\
300 \\
300\end{array}$ & $\begin{array}{l}\mathrm{N} \\
\mathrm{N} \\
\mathrm{N} \\
\mathrm{N} \\
\mathrm{N}\end{array}$ & $\begin{array}{r}2,000 \\
10 \\
\mathrm{~N} \\
\mathrm{~N} \\
\mathrm{~N}\end{array}$ & $\begin{array}{r}50 \\
300 \\
700 \\
700 \\
700\end{array}$ & $\begin{array}{l}\text { I. } \\
\text { L } \\
L \\
L\end{array}$ & $\begin{array}{r}7 \\
150 \\
10 \\
L \\
7\end{array}$ & $\begin{array}{r}L \\
150 \\
7 \\
L \\
5\end{array}$ & $\begin{array}{r}20 \\
50 \\
10 \\
7 \\
20\end{array}$ & $\begin{array}{l}N \\
N \\
L \\
L \\
L\end{array}$ & $\begin{array}{l}\mathrm{N} \\
\mathrm{N} \\
\mathrm{N} \\
\mathrm{N} \\
\mathrm{N}\end{array}$ \\
\hline $\begin{array}{l}11-A \\
12-S \\
13-A \\
14-5 \\
15-A\end{array}$ & $\begin{array}{l}7 \\
1.5 \\
1.5 \\
1.5 \\
2\end{array}$ & $\begin{array}{l}3 \\
.3 \\
.3 \\
.5 \\
.3\end{array}$ & $\begin{array}{l}7 \\
1.5 \\
1.5 \\
1.5 \\
1.5\end{array}$ & $\begin{array}{l}.7 \\
.2 \\
.15 \\
.2 \\
.15\end{array}$ & $\begin{array}{l}500 \\
300 \\
300 \\
300 \\
150\end{array}$ & $\begin{array}{l}N \\
N \\
N \\
N \\
N\end{array}$ & $\begin{array}{l}15 \\
N \\
N \\
L \\
N\end{array}$ & $\begin{array}{r}500 \\
1,000 \\
300 \\
500 \\
2,000\end{array}$ & $\begin{array}{l}\mathrm{N} \\
\mathrm{L} \\
\mathrm{L} .5 \\
\mathrm{~L}\end{array}$ & $\begin{array}{r}50 \\
L \\
N \\
10 \\
5\end{array}$ & $\begin{array}{l}5 \\
5 \\
L \\
5 \\
L\end{array}$ & $\begin{array}{l}30 \\
10 \\
10 \\
20 \\
30\end{array}$ & $\begin{array}{r}L \\
20 \\
30 \\
N \\
20\end{array}$ & $\begin{array}{l}\mathrm{N} \\
\mathrm{N} \\
\mathrm{N} \\
\mathrm{N} \\
\mathrm{N}\end{array}$ \\
\hline $\begin{array}{l}16-5 \\
17-5 \\
18-5 \\
19 a-R \\
19 b-R\end{array}$ & $\begin{array}{l}2 \\
1.5 \\
1.5 \\
3 \\
.7\end{array}$ & $\begin{array}{l}.5 \\
.5 \\
.3 \\
. \quad .07\end{array}$ & $\begin{array}{l}1.5 \\
1.5 \\
1.5 \\
1.5 \\
.15\end{array}$ & $\begin{array}{l}.2 \\
.2 \\
.15 \\
.3 \\
.03\end{array}$ & $\begin{array}{r}300 \\
300 \\
200 \\
500 \\
70\end{array}$ & $\begin{array}{l}N \\
N \\
N \\
N \\
N\end{array}$ & $\begin{array}{l}L \\
N \\
N \\
N \\
N\end{array}$ & $\begin{array}{r}1,500 \\
700 \\
1,500 \\
200 \\
30\end{array}$ & $\begin{array}{l}1 \\
1 \\
L \\
N \\
N\end{array}$ & $\begin{array}{r}10 \\
10 \\
7 \\
20 \\
N\end{array}$ & $\begin{array}{l}7 \\
5 \\
7 \\
7 \\
L\end{array}$ & $\begin{array}{l}15 \\
15 \\
20 \\
10 \\
10\end{array}$ & $\begin{array}{r}\mathrm{L} \\
20 \\
\mathrm{~N} \\
\mathrm{~N} \\
\mathrm{~N}\end{array}$ & $\begin{array}{l}\mathrm{N} \\
\mathrm{N} \\
\mathrm{N} \\
\mathrm{N} \\
\mathrm{N}\end{array}$ \\
\hline $\begin{array}{l}20-5 \\
21-S \\
22-S \\
23-R \\
24-R\end{array}$ & $\begin{array}{l}1.5 \\
2 \\
1.5 \\
3 \\
.3\end{array}$ & $\begin{array}{l}.5 \\
.2 \\
.2 \\
.7 \\
.02\end{array}$ & $\begin{array}{l}1.5 \\
1 \\
1 \\
3 \\
.1\end{array}$ & $\begin{array}{l}.2 \\
.2 \\
.2 \\
.3 \\
.2\end{array}$ & $\begin{array}{l}300 \\
200 \\
200 \\
300 \\
300\end{array}$ & $\begin{array}{l}\mathrm{N} \\
\mathrm{N} \\
\mathrm{N} \\
\mathrm{N} \\
\mathrm{N}\end{array}$ & $\begin{array}{l}L \\
N \\
N \\
L \\
N\end{array}$ & $\begin{array}{r}700 \\
700 \\
300 \\
700 \\
50\end{array}$ & $\begin{array}{l}1.5 \\
L \\
L \\
L \\
L\end{array}$ & $\begin{array}{r}7 \\
5 \\
7 \\
10 \\
N\end{array}$ & $\begin{array}{l}5 \\
L \\
L \\
7 \\
5\end{array}$ & $\begin{array}{l}30 \\
10 \\
15 \\
30 \\
10\end{array}$ & $\begin{array}{r}20 \\
50 \\
N \\
N \\
N\end{array}$ & $\begin{array}{l}\mathrm{N} \\
\mathrm{N} \\
\mathrm{N} \\
\mathrm{N} \\
\mathrm{N}\end{array}$ \\
\hline $\begin{array}{l}25-5 \\
26-5 \\
27-5 \\
28-5 \\
29-5\end{array}$ & $\begin{array}{l}2 \\
1.5 \\
1.5 \\
1.5 \\
1.5\end{array}$ & $\begin{array}{l}.7 \\
.7 \\
.3 \\
.3 \\
.3\end{array}$ & $\begin{array}{l}3 \\
2 \\
1.5 \\
1.5 \\
1.5\end{array}$ & $\begin{array}{l}.3 \\
.15 \\
.2 \\
.2 \\
.3\end{array}$ & $\begin{array}{l}500 \\
300 \\
300 \\
300 \\
300\end{array}$ & $\begin{array}{l}\mathrm{N} \\
\mathrm{N} \\
\mathrm{N} \\
\mathrm{N} \\
\mathrm{N}\end{array}$ & $\begin{array}{l}\mathrm{N} \\
\mathrm{N} \\
\mathrm{N} \\
\mathrm{N} \\
\mathrm{N}\end{array}$ & $\begin{array}{r}500 \\
1,000 \\
700 \\
700 \\
700\end{array}$ & $\begin{array}{l}1.5 \\
L \\
1 \\
L \\
1\end{array}$ & $\begin{array}{l}7 \\
7 \\
7 \\
5 \\
5\end{array}$ & $\begin{array}{r}10 \\
15 \\
5 \\
7 \\
7\end{array}$ & $\begin{array}{r}15 \\
3 \\
7 \\
10 \\
15\end{array}$ & $\begin{array}{r}20 \\
N \\
N \\
20 \\
L\end{array}$ & $\begin{array}{l}\mathrm{N} \\
\mathrm{N} \\
\mathrm{N} \\
\mathrm{N} \\
\mathrm{N}\end{array}$ \\
\hline $\begin{array}{l}30-5 \\
31-5 \\
32-5 \\
33-5 \\
34-5\end{array}$ & $\begin{array}{r}1 \\
1 \\
.7 \\
1.5\end{array}$ & $\begin{array}{l}.3 \\
.3 \\
.3 \\
.3 \\
.3\end{array}$ & $\begin{array}{l}1.5 \\
1.5 \\
1.5 \\
1.5 \\
1.5\end{array}$ & $\begin{array}{l}.15 \\
.2 \\
.15 \\
.15 \\
.3\end{array}$ & $\begin{array}{l}300 \\
300 \\
200 \\
200 \\
300\end{array}$ & $\begin{array}{l}\mathrm{N} \\
\mathrm{N} \\
\mathrm{N} \\
\mathrm{N} \\
\mathrm{N}\end{array}$ & $\begin{array}{l}N \\
N \\
N \\
N \\
N\end{array}$ & $\begin{array}{l}500 \\
500 \\
700 \\
700 \\
700\end{array}$ & $\begin{array}{l}1 \\
L \\
L \\
1 \\
1\end{array}$ & $\begin{array}{l}5 \\
5 \\
5 \\
L \\
5\end{array}$ & $\begin{array}{l}L \\
L \\
L \\
L \\
5\end{array}$ & $\begin{array}{r}15 \\
5 \\
10 \\
7 \\
10\end{array}$ & $\begin{array}{l}N \\
N \\
N \\
N \\
L\end{array}$ & $\begin{array}{l}N \\
N \\
L \\
N \\
N\end{array}$ \\
\hline $\begin{array}{l}35-5 \\
36-5 \\
37-5 \\
38-5 \\
39-5\end{array}$ & $\begin{array}{l}1.5 \\
1 \\
1.5 \\
1.5 \\
1.5\end{array}$ & $\begin{array}{l}.3 \\
.3 \\
.3 \\
.5 \\
.3\end{array}$ & $\begin{array}{l}1.5 \\
1.5 \\
1.5 \\
1.5 \\
1.5\end{array}$ & $\begin{array}{l}.3 \\
.2 \\
.15 \\
.2 \\
.2\end{array}$ & $\begin{array}{l}300 \\
300 \\
300 \\
300 \\
300\end{array}$ & $\begin{array}{l}\mathrm{N} \\
\mathrm{N} \\
\mathrm{N} \\
\mathrm{N} \\
\mathrm{N}\end{array}$ & $\begin{array}{l}N \\
N \\
N \\
N \\
N\end{array}$ & $\begin{array}{r}1,000 \\
700 \\
1,000 \\
700 \\
700\end{array}$ & $\begin{array}{l}1 \\
1 \\
1 \\
1 \\
1\end{array}$ & $\begin{array}{l}5 \\
5 \\
5 \\
7 \\
5\end{array}$ & $\begin{array}{l}5 \\
5 \\
5 \\
5 \\
L\end{array}$ & $\begin{array}{l}10 \\
10 \\
10 \\
15 \\
15\end{array}$ & $\begin{array}{l}\mathrm{L} \\
\mathrm{L} \\
\mathrm{N} \\
\mathrm{N} \\
\mathrm{N}\end{array}$ & $\begin{array}{l}N \\
N \\
N \\
N \\
N\end{array}$ \\
\hline $\begin{array}{l}40-5 \\
41-5 \\
42-5 \\
43-5 \\
44-5\end{array}$ & $\begin{array}{l}1 \\
1.5 \\
1.5 \\
2\end{array}$ & $\begin{array}{l}.3 \\
.3 \\
.2 \\
.2 \\
.3\end{array}$ & $\begin{array}{l}1.5 \\
1 \\
1.5 \\
.7 \\
1.5\end{array}$ & $\begin{array}{l}.07 \\
.15 \\
.2 \\
.2 \\
.15\end{array}$ & $\begin{array}{l}300 \\
200 \\
300 \\
200 \\
300\end{array}$ & $\begin{array}{l}N \\
N \\
N \\
N \\
N\end{array}$ & $\begin{array}{l}N \\
N \\
N \\
N \\
N\end{array}$ & $\begin{array}{r}1,000 \\
700 \\
700 \\
700 \\
1,000\end{array}$ & $\begin{array}{l}1.5 \\
L \\
L \\
L \\
L\end{array}$ & $\begin{array}{l}\text { A } \\
5 \\
N \\
L \\
7\end{array}$ & $\begin{array}{l}\mathrm{L} \\
\mathrm{L} \\
\mathrm{L} \\
\mathrm{L} \\
5\end{array}$ & $\begin{array}{r}7 \\
10 \\
15 \\
7 \\
7\end{array}$ & $\begin{array}{l}N \\
N \\
L \\
N \\
N\end{array}$ & $\begin{array}{l}\mathrm{N} \\
\mathrm{N} \\
\mathrm{N} \\
\mathrm{N} \\
\mathrm{L}\end{array}$ \\
\hline $\begin{array}{l}45-S \\
46-S \\
46 b-S \\
47-S \\
48-A\end{array}$ & $\begin{array}{l}3 \\
.7 \\
1.5 \\
1\end{array}$ & $\begin{array}{l}.7 \\
.15 \\
.15 \\
.15 \\
.07\end{array}$ & $\begin{array}{l}1.5 \\
1 \\
1 \\
1.5 \\
.7\end{array}$ & $\begin{array}{l}.3 \\
.1 \\
.3 \\
.1 \\
.07\end{array}$ & $\begin{array}{l}300 \\
200 \\
300 \\
150 \\
150\end{array}$ & $\begin{array}{l}N \\
N \\
N \\
N \\
N\end{array}$ & $\begin{array}{l}N \\
N \\
N \\
N \\
N\end{array}$ & $\begin{array}{r}700 \\
1,000 \\
1,000 \\
1,000 \\
700\end{array}$ & $\begin{array}{l}\mathrm{L} \\
\mathrm{L} \\
\mathrm{N} \\
1 \\
1\end{array}$ & $\begin{array}{c}10 \\
N \\
L \\
L \\
N\end{array}$ & $\begin{array}{l}5 \\
L \\
L \\
L \\
L\end{array}$ & $\begin{array}{r}15 \\
3 \\
7 \\
5 \\
10\end{array}$ & $\begin{array}{c}N \\
L \\
30 \\
L \\
N\end{array}$ & $\begin{array}{l}N \\
N \\
N \\
N \\
N\end{array}$ \\
\hline
\end{tabular}

See footnotes at end of table. 


\section{Emigrant Basin primitive area, California}

designations: Grn=granitic rocks; Vol=volcanic rocks; Met=metamorphosed and (or) mlxed rocks; Qal=unconsolidated deposit; Alt=altered rock zone; and Dio $=$ diorite dike rock. Analysts: D. J. Grimes, R. H. Heldel, and J. M. Motooka (six-step semiquantitative spectrographic analyses); W. L. Campbell, E. J. Fennelly, S. L. Noble, M. S. Rickard, J. A. Thomas, R. B. Tripp, and R. C. Yager (atomic absorption, $\mathrm{Au}, \mathrm{Cu}, \mathrm{Pb}, \mathrm{Zn}$ ) ; J. G. Frisken (colorimetry, Mo, W) ; s. L. Noble (instrumental Hg detection); and G. W. Dounay and S. L. Noble (paper chromatography, U) ].

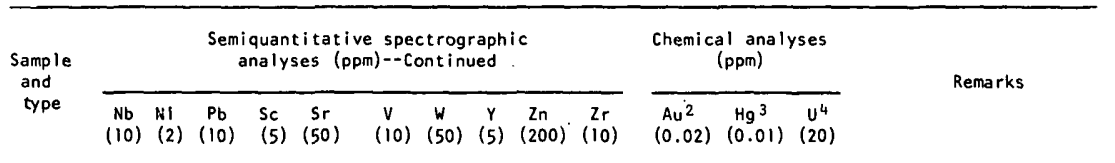

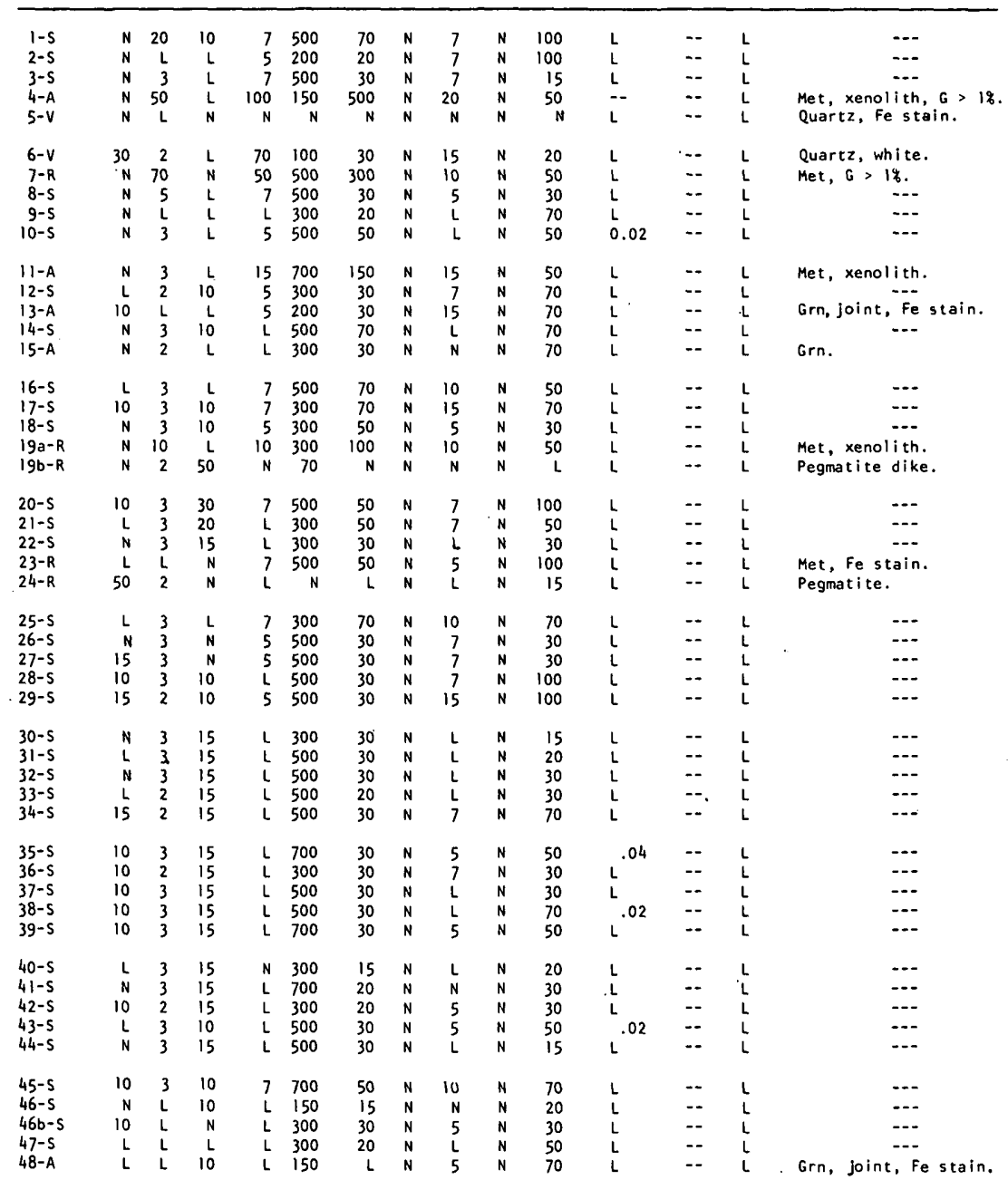


TABLE 4.-Analyses of samples from the Emigrant

\begin{tabular}{|c|c|c|c|c|c|c|c|c|c|c|c|c|c|c|}
\hline \multirow{2}{*}{$\begin{array}{l}\text { Sample } \\
\text { and } \\
\text { type }\end{array}$} & \multicolumn{4}{|c|}{$\begin{array}{c}\text { Semiquantitative spectrographic } \\
\text { anaiyses (percent) }\end{array}$} & \multicolumn{10}{|c|}{$\begin{array}{c}\text { Semiquantitative spectrographic } \\
\text { analyses }(\mathrm{ppm})^{3}\end{array}$} \\
\hline & $\begin{array}{c}\mathrm{Fe} \\
(0.05)\end{array}$ & $\begin{array}{c}\mathrm{Mg} \\
(0.005)\end{array}$ & $\begin{array}{c}\mathrm{Ca} \\
(0.05)\end{array}$ & $\begin{array}{c}T i \\
(0.001)\end{array}$ & $\begin{array}{c}\mathrm{Mn} \\
(20)\end{array}$ & $\begin{array}{l}\mathrm{Ag} \\
(0.5)\end{array}$ & $\stackrel{8}{(10)}$ & $\begin{array}{c}B a \\
(20)\end{array}$ & $\begin{array}{l}\mathrm{Be} \\
\text { (1) }\end{array}$ & $\begin{array}{l}\text { Co } \\
\text { (5) }\end{array}$ & $\begin{array}{l}\mathrm{Cr} \\
(5)\end{array}$ & $\begin{array}{l}\mathrm{Cu} \\
\text { (2) }\end{array}$ & $\begin{array}{l}\text { La } \\
(20)\end{array}$ & $\begin{array}{l}\text { Mo } \\
(5)\end{array}$ \\
\hline $49-5$ & 1 & 0.2 & 1 & 0.15 & 300 & $\mathrm{~N}$ & N & 700 & L & $L$ & L & 7 & $\mathrm{~N}$ & $\mathrm{~N}$ \\
\hline $50-A$ & 3 & .3 & .5 & .3 & 300 & $\mathrm{~N}$ & $\mathrm{~L}$ & 700 & 1 & 5 & 300 & 30 & 50 & 5 \\
\hline $51-5$ & 1.5 & .3 & 1.5 & .2 & 300 & $\mathrm{~N}$ & $\mathrm{~N}$ & 700 & 1 & 7 & 7 & 10 & $N$ & $\mathrm{~N}$ \\
\hline $52-5$ & 1.5 & .3 & 1 & .2 & 200 & $\mathrm{~N}$ & $N$ & 700 & L & 5 & 5 & 7 & 30 & $\mathrm{~N}$ \\
\hline $53-5$ & 1.5 & .2 & $i$ & .2 & 200 & N & $N$ & 1,000 & L & $\hat{L}$ & $\hat{L}$ & 10 & $\mathrm{~N}$ & $\mathrm{~N}$ \\
\hline $54-5$ & 1.5 & .3 & 1.5 & .3 & 300 & N & $N$ & 700 & L & 7 & 5 & 7 & L & L \\
\hline $55-\mathrm{s}$ & 1.5 & .2 & 1 & .15 & 200 & $\mathrm{~N}$ & $N$ & 700 & L & 5 & 5 & 5 & N & $N$ \\
\hline $56-5$ & 1.5 & .3 & 1.5 & .3 & 500 & $\mathrm{~N}$ & $\mathrm{~N}$ & 300 & 1 & 7 & $\mathrm{~L}$ & 10 & 20 & 5 \\
\hline $57-5$ & 1.5 & .2 & 1 & .15 & 200 & $\mathrm{~N}$ & $\mathrm{~N}$ & 1,000 & L & 5 & 5 & 5 & $N$ & L \\
\hline $58-5$ & 2 & .3 & 1.5 & .2 & 300 & $\mathrm{~N}$ & $N$ & 700 & L & 5 & 7 & 15 & $\mathrm{~L}$ & $N$ \\
\hline $59-5$ & 1.5 & .3 & 1.5 & .15 & 300 & N & $\mathrm{N}$ & 500 & 1.5 & 5 & 5 & 5 & $L$ & $N$ \\
\hline $60-5$ & 3 & .7 & 1.5 & .3 & 300 & N & $N$ & 700 & $L$ & 15 & 30 & 20 & L & $\mathrm{N}$ \\
\hline $61-5$ & 3 & .7 & 2 & .3 & 500 & $\mathrm{~N}$ & 10 & 1,500 & 1 & 15 & 30 & 30 & 20 & $\mathrm{~N}$ \\
\hline $62-A$ & 1.5 & .2 & 1.5 & .2 & 300 & $\mathrm{~N}$ & $\mathrm{~N}$ & 700 & 1 & 5 & $L$ & 10 & $L$ & $\mathrm{~N}$ \\
\hline $63 a-8$ & 2 & .7 & 2 & .3 & 300 & $\mathrm{~N}$ & $\mathrm{~N}$ & 1,000 & L & 7 & 10 & 30 & $L$ & $\mathrm{~N}$ \\
\hline $63 b-R$ & 3 & .7 & 2 & .5 & 300 & N & L & 1,000 & L & 15 & 15 & 50 & 30 & N \\
\hline $64-R$ & 3 & .3 & 1.5 & .3 & 300 & 0.7 & $N$ & 1,000 & L & 7 & 5 & L & 20 & $N$ \\
\hline $65 a-R$ & 3 & .7 & 2 & .3 & 300 & $\mathrm{~N}$ & $\mathrm{~N}$ & 700 & $L$ & 15 & 15 & 50 & L & $N$ \\
\hline $65 \mathrm{~b}-\mathrm{R}$ & 2 & .7 & 1 & .2 & 300 & $\mathrm{~N}$ & N & 500 & $\mathrm{~N}$ & 10 & 7 & 30 & $N$ & $\mathrm{~N}$ \\
\hline $66-5$ & 3 & 1 & 5 & .3 & 700 & $\mathrm{~N}$ & 10 & 1,500 & L & 15 & 50 & 30 & 20 & N \\
\hline $67-K$ & 3 & 5 & 7 & .3 & 700 & $\mathrm{~N}$ & N & 300 & N & 50 & 100 & 30 & N & N \\
\hline $68-5$ & 3 & .7 & 1.5 & .3 & 500 & $\mathrm{~N}$ & N & 1,000 & L & 15 & 30 & 20 & $\mathrm{~N}$. & 5 \\
\hline $69-5$ & 3 & 1 & 3 & .5 & 500 & $\mathrm{~N}$ & 10 & 1,500 & $L$ & 15 & 30 & 30 & 20 & N \\
\hline $70-5$ & 3 & 1 & 2 & .5 & 500 & $\mathrm{~N}$ & $\mathrm{~L}$ & 1,500 & L & 15 & 30 & 30 & L & $N$ \\
\hline $71-5$ & 3 & .7 & 2 & .5 & 500 & N & $\mathrm{L}$ & 1,500 & L & 10 & 30 & 30 & $\mathrm{~L}$ & $\mathrm{~N}$ \\
\hline $72-5$ & 3 & .7 & 2 & .5 & 300 & $\mathrm{~N}$ & $\mathrm{~N}$ & 1,000 & L & 15 & 50 & 30 & L & $\mathrm{N}$ \\
\hline $73-5$ & 2 & .7 & 1.5 & .5 & 300 & $\mathrm{~N}$ & $N$ & 1,500 & L & 10 & 30 & 20 & L & $\mathrm{N}$ \\
\hline $74-5$ & 1.5 & .3 & 1.5 & .15 & 300 & $\mathrm{~N}$ & $N$ & 1,000 & 1 & 5 & L & 7 & 20 & $N$ \\
\hline $75-s$ & 2 & .5 & 1.5 & .3 & 300 & $\mathrm{~N}$ & $\mathrm{~N}$ & 1,000 & L & 10 & 10 & 15 & $L$ & $\mathrm{~N}$ \\
\hline $76-5$ & 3 & 1 & 2 & .7 & 300 & $\mathrm{~N}$ & $\mathrm{~N}$ & 1,000 & L & 20 & 150 & 30. & $\mathrm{~L}$ & N \\
\hline $77-5$ & 3 & 1 & 1.5 & .3 & 300 & N & N & 700 & L & 15 & 70 & 15 & L & $N$ \\
\hline $78-5$ & 2 & .7 & 1.5 & .3 & 300 & $\mathrm{~N}$ & $\mathrm{~N}$ & 1,000 & L & 15 & 30 & 20 & L & $\mathrm{N}$ \\
\hline $79-5$ & 2 & .7 & 2 & .3 & 300 & $\mathrm{~N}$ & N & 1,000 & $L$ & 15 & 30 & 20 & 20 & $\mathrm{~N}$ \\
\hline $80-5$ & 2 & .5 & 1.5 & .3 & 300 & $\mathrm{~N}$ & $\mathrm{~N}$ & 1,000 & L & 10 & 30 & 15 & $L$ & $\mathrm{~N}$ \\
\hline $81-5$ & 1.5 & .5 & 1.5 & .3 & 300 & N & $N$ & 700 & L & 7 & 7 & 10 & $L$ & N \\
\hline $82-5$ & 1.5 & .3 & 1.5 & .2 & 300 & $N$ & $\mathrm{~N}$ & 1,500 & 1 & $\mathrm{~L}$ & L & 7 & 30 & $\mathrm{~N}$ \\
\hline $83-R$ & 2 & .3 & 1.5 & .3 & 300 & N & $\mathrm{N}$ & 1,500 & $L$ & 5 & L & 7 & 20 & $\mathrm{~N}$ \\
\hline $84-5$ & 1.5 & .2 & 1 & .2 & 200 & N & $N$ & 1.500 & $L$ & 5 & L & 7 & $L$ & $\mathrm{~N}$ \\
\hline $85-5$ & 1.5 & .2 & 1.5 & .2 & 200 & $\mathrm{~N}$ & N & 1,500 & L & $\grave{i}$ & $L$ & 7 & 20 & $\mathrm{~N}$ \\
\hline $86-5$ & 2 & .5 & 1 & .3 & 300 & N & $\mathrm{N}$ & 700 & L & 7 & 10 & 10 & L & L \\
\hline $87-5$ & 2 & .5 & 1.5 & .3 & 300 & $\mathrm{~N}$ & $\mathrm{~N}$ & 500 & L & 10 & 7 & 10 & 20 & $\mathrm{~N}$ \\
\hline $88-5$ & 1.5 & .3 & 1.5 & .2 & 200 & $\mathrm{~N}$ & $\mathrm{~N}$ & 700 & 1 & 5 & $\mathrm{~L}$ & 10 & $L$ & N \\
\hline $89-5$ & 2 & .7 & 1 & .3 & 300 & N & L & 300 & L & 7 & 5 & 15 & $\mathrm{~N}$ & $\mathrm{~N}$ \\
\hline $90-5$ & 1.5 & .3 & 1 & .15 & 200 & N & $\mathrm{N}$ & 700 & L & 5 & L & 2 & $\mathrm{~N}$ & $\mathrm{~N}$ \\
\hline $91-v$ & L & .03 & L & .01 & L & N & $\mathrm{N}$ & 50 & N & $\mathrm{N}$ & L & $i$ & $\mathrm{~N}$ & N \\
\hline $92-5$ & 3 & .7 & 1.5 & .3 & 300 & N & L & 500 & L & 10 & 30 & 20 & N & N \\
\hline $93-5$ & 3 & .7 & 1.5 & .3 & 300 & $\mathrm{~N}$ & $L$ & 700 & L & .10 & 30 & 30 & $N$ & $\mathrm{~N}$ \\
\hline $94-5$ & 1.5 & .2 & 1.5 & .15 & 150 & $\mathrm{~N}$ & $N$ & 700 & 1 & $L$ & $\mathrm{~L}$ & 5 & 20 & $\mathrm{~N}$ \\
\hline $95-5$ & 1.5 & .2 & 1.5 & .15 & 150 & $\mathrm{~N}$ & $\mathrm{~N}$ & 700 & L & 5 & 5 & 10 & $\mathrm{~N}$ & $\mathrm{~N}$ \\
\hline $96-5$ & 1.5 & .2 & 1 & .15 & 200 & $\mathrm{~N}$ & $\mathrm{~N}$ & 700 & L & L & L & 5 & $L$ & $\mathrm{~N}$ \\
\hline $97-c$ & & & 5 & .7 & 700 & N & 10 & 700 & L & 30 & 300 & 212 & 30 & ${ }^{5} L(2)$ \\
\hline $98-5$ & . 1.5 & .3 & 1.5 & .3 & 150 & $\mathrm{~N}$ & $L$ & 500 & 1.5 & L & 7 & ${ }^{26} 6$ & 30 & $5 \mathrm{~L}(2)$ \\
\hline $99-R$ & 1.5 & .5 & 1.5 & .5 & 500 & N & L & 700 & 1 & 10 & $\mathrm{~L}$ & 211 & 70 & $5 L(2)$ \\
\hline $100-5$ & 1.5 & .3 & 1.5 & .3 & 150 & $\mathrm{~N}$ & $\mathrm{~L}$ & 700 & L & 7 & 5 & 10 & $\mathrm{~L}$ & $\mathrm{~N}$ \\
\hline $101-5$ & 3 & .7 & 2 & .5 & 500 & $\mathrm{~N}$ & $\mathrm{~N}$ & 500 & 1 & 10 & 10 & 20 & $\mathrm{~N}$ & $\mathrm{~N}$ \\
\hline $102-5$ & 1.5 & .3 & 1.5 & .1 & 150 & N & $\mathrm{N}$ & 1,000 & L & L & L & 3 & L & $\mathrm{N}$ \\
\hline $103-5$ & 1.5 & .3 & 1.5 & .15 & 300 & $\mathrm{~N}$ & $\mathrm{~N}$ & 1,000 & 1 & 5 & $L$ & 10 & 20 & N \\
\hline $104-5$ & 1.5 & .3 & 1.5 & .1 & 300 & $N$ & $\mathrm{~N}$ & 700 & 1 & L & 5 & 5 & 30 & N \\
\hline $105-5$ & 1.5 & .3 & 1.5 & .15 & 300 & $\mathrm{~N}$ & $\mathrm{~N}$ & 700 & 1.5 & 5 & 5 & 7 & $L$ & L \\
\hline $106-5$ & 1.5 & .3 & 1.5 & .15 & 300 & $N$ & N & 1,000 & 1 & $i$ & $\mathrm{~L}$ & 5 & 20 & $\mathrm{~N}$ \\
\hline
\end{tabular}


EMIGRANT BASIN PRIMITIVE AREA, CALIFORNIA

Basin primitive area, California-Continued

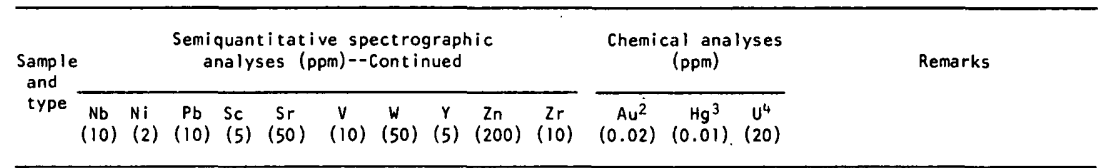

\begin{tabular}{|c|c|c|c|c|c|c|c|c|c|c|c|c|c|c|}
\hline $\begin{array}{l}49-S \\
50-A \\
51-S \\
52-S \\
53-S\end{array}$ & $\begin{array}{r}N \\
10 \\
N \\
L \\
10\end{array}$ & $\begin{array}{r}L \\
30 \\
L \\
L \\
L\end{array}$ & $\begin{array}{l}L \\
15 \\
15 \\
15 \\
15\end{array}$ & $\begin{array}{l}\mathrm{L} \\
7 \\
\mathrm{~L} \\
\mathrm{~L} \\
\mathrm{~N}\end{array}$ & $\begin{array}{l}300 \\
300 \\
300 \\
300 \\
300\end{array}$ & $\begin{array}{l}20 \\
50 \\
50 \\
50 \\
50\end{array}$ & $\begin{array}{l}\mathrm{N} \\
\mathrm{N} \\
\mathrm{N} \\
\mathrm{N} \\
\mathrm{N}\end{array}$ & $\begin{array}{r}\mathrm{L} \\
15 \\
5 \\
\mathrm{~L} \\
\mathrm{~L}\end{array}$ & $\begin{array}{l}N \\
N \\
N \\
N \\
N\end{array}$ & $\begin{array}{l}15 \\
70 \\
70 \\
30 \\
50\end{array}$ & $\begin{array}{l}\mathrm{L} \\
\mathrm{L} \\
\mathrm{L} \\
\mathrm{L} \\
\mathrm{L}\end{array}$ & $\begin{array}{l}-- \\
-- \\
-- \\
--\end{array}$ & $\begin{array}{l}L \\
L \\
L \\
L \\
L\end{array}$ & Met, Fe stain. $\begin{array}{r} \\
\cdots \\
\\
\cdots\end{array}$ \\
\hline $\begin{array}{l}54-5 \\
55-5 \\
56-5 \\
57-5 \\
58-5\end{array}$ & $\begin{array}{r}10 \\
L \\
10 \\
L \\
10\end{array}$ & $\begin{array}{l}3 \\
2 \\
3 \\
L \\
L\end{array}$ & $\begin{array}{l}15 \\
15 \\
20 \\
20 \\
20\end{array}$ & $\begin{array}{l}L \\
L \\
L \\
L \\
L\end{array}$ & $\begin{array}{l}300 \\
500 \\
300 \\
700 \\
700\end{array}$ & $\begin{array}{l}50 \\
30 \\
50 \\
30 \\
50\end{array}$ & $\begin{array}{l}\mathrm{N} \\
\mathrm{N} \\
\mathrm{N} \\
\mathrm{N} \\
\mathrm{N}\end{array}$ & $\begin{array}{c}7 \\
L \\
10 \\
L \\
5\end{array}$ & $\begin{array}{l}\mathbf{N} \\
N \\
N \\
N \\
N\end{array}$ & $\begin{array}{l}20 \\
15 \\
70 \\
50 \\
50\end{array}$ & $\begin{array}{l}L \\
L \\
0.02 \\
L .02\end{array}$ & $\begin{array}{l}-- \\
-- \\
-- \\
--\end{array}$ & $\begin{array}{l}L \\
L \\
L \\
L \\
L\end{array}$ & $\begin{array}{l}\ldots \\
\cdots \\
-\ldots \\
-\cdots\end{array}$ \\
\hline $\begin{array}{l}59-S \\
60-S \\
61-S \\
62-A \\
63 a-R\end{array}$ & $\begin{array}{r}L \\
10 \\
10 \\
10 \\
L\end{array}$ & $\begin{array}{r}L \\
30 \\
15 \\
L \\
7\end{array}$ & $\begin{array}{l}10 \\
10 \\
15 \\
L \\
L\end{array}$ & $\begin{array}{l}\mathrm{L} \\
7 \\
7 \\
\mathrm{~L} \\
7\end{array}$ & $\begin{array}{r}500 \\
700 \\
1,000 \\
200 \\
2,000\end{array}$ & $\begin{array}{l}30 \\
70 \\
50 \\
20 \\
70\end{array}$ & $\begin{array}{l}N \\
N \\
N \\
N \\
N\end{array}$ & $\begin{array}{r}N \\
5 \\
7 \\
15 \\
5\end{array}$ & $\begin{array}{l}\mathrm{N} \\
\mathrm{N} \\
\mathrm{N} \\
\mathrm{N} \\
\mathrm{N}\end{array}$ & $\begin{array}{r}20 \\
150 \\
200 \\
150 \\
100\end{array}$ & $\begin{array}{l}L^{.02} \\
L \\
L^{*} .04\end{array}$ & $\begin{array}{l}-- \\
-- \\
-- \\
-- \\
--\end{array}$ & $\begin{array}{l}L \\
L \\
L \\
L \\
L\end{array}$ & $\begin{array}{l}\text { Gr } \\
\text { Grn, joint surface, *5 g sample. } \\
\text { Vol, agglomerate clast. }\end{array}$ \\
\hline $\begin{array}{l}63 b-R \\
64-R \\
65 a-R \\
65 b-R \\
66-S\end{array}$ & $\begin{array}{l}\mathrm{L} \\
\mathrm{L} \\
\mathrm{L} \\
\mathrm{N} \\
\mathrm{L}\end{array}$ & $\begin{array}{r}20 \\
L \\
20 \\
5 \\
20\end{array}$ & $\begin{array}{r}\mathrm{L} \\
20 \\
10 \\
\mathrm{~L} \\
\mathrm{~L}\end{array}$ & $\begin{array}{r}10 \\
5 \\
10 \\
7 \\
7\end{array}$ & $\begin{array}{r}1,500 \\
500 \\
1,000 \\
700 \\
1,000\end{array}$ & $\begin{array}{r}100 \\
30 \\
70 \\
50 \\
70\end{array}$ & $\begin{array}{l}\mathrm{N} \\
\mathrm{N} \\
\mathrm{N} \\
\mathrm{N} \\
\mathrm{N}\end{array}$ & $\begin{array}{r}15 \\
10 \\
7 \\
5 \\
7\end{array}$ & $\begin{array}{l}\mathbf{N} \\
\mathbf{N} \\
\mathrm{N} \\
\mathrm{N} \\
\mathrm{N}\end{array}$ & $\begin{array}{r}100 \\
70 \\
150 \\
70 \\
100\end{array}$ & $\begin{array}{l}\mathrm{L} \\
\mathrm{L} \\
\mathrm{L} \\
\mathrm{L} \\
\mathrm{L}\end{array}$ & $\begin{array}{l}-- \\
-- \\
-- \\
-- \\
--\end{array}$ & $\begin{array}{l}\mathrm{L} \\
\mathrm{L} \\
\mathrm{L} \\
\mathrm{L} \\
\mathrm{L}\end{array}$ & $\begin{array}{l}\text { Vol, matrix. } \\
\text { Grn, near Vol contact. } \\
\text { Vol, clast, green. } \\
\text { Vol, clast, red. }\end{array}$ \\
\hline $\begin{array}{l}67-R \\
68-S \\
69-5 \\
70-5 \\
71-5\end{array}$ & $\begin{array}{c}N \\
N \\
10 \\
L \\
L\end{array}$ & $\begin{array}{l}50 \\
10 \\
20 \\
30 \\
20\end{array}$ & $\begin{array}{l}N \\
L \\
10 \\
15 \\
15\end{array}$ & $\begin{array}{r}15 \\
7 \\
10 \\
10 \\
7\end{array}$ & $\begin{array}{r}700 \\
700 \\
1,000 \\
1,000 \\
1,000\end{array}$ & $\begin{array}{l}70 \\
70 \\
70 \\
70 \\
70\end{array}$ & $\begin{array}{l}N \\
N \\
N \\
N \\
N\end{array}$ & $\begin{array}{l}7 \\
7 \\
7 \\
7 \\
7\end{array}$ & $\begin{array}{l}N \\
N \\
N \\
N \\
N\end{array}$ & $\begin{array}{r}50 \\
70 \\
100 \\
100 \\
70\end{array}$ & $L^{.04}$ & $\begin{array}{l}-- \\
-- \\
-- \\
--\end{array}$ & $\begin{array}{l}\mathrm{L} \\
\mathrm{L} \\
\mathrm{L} \\
\mathrm{L} \\
\mathrm{L}\end{array}$ & $\begin{array}{l}\cdots \\
\cdots \\
\cdots\end{array}$ \\
\hline $\begin{array}{l}72-5 \\
73-5 \\
74-5 \\
75-5 \\
76-5\end{array}$ & $\begin{array}{r}\mathbf{L} \\
\mathbf{L} \\
\mathbf{L} \\
\mathbf{L} \\
10\end{array}$ & $\begin{array}{r}30 \\
20 \\
L \\
7 \\
30\end{array}$ & $\begin{array}{l}10 \\
15 \\
15 \\
10 \\
15\end{array}$ & $\begin{array}{r}7 \\
7 \\
5 \\
7 \\
15\end{array}$ & $\begin{array}{r}700 \\
700 \\
300 \\
500 \\
1,000\end{array}$ & $\begin{array}{l}70 \\
70 \\
20 \\
50 \\
70\end{array}$ & $\begin{array}{l}\mathbf{N} \\
\mathrm{N} \\
\mathrm{N} \\
\mathrm{N} \\
\mathrm{N}\end{array}$ & $\begin{array}{l}7 \\
7 \\
5 \\
7 \\
7\end{array}$ & $\begin{array}{l}N \\
N \\
N \\
N \\
N\end{array}$ & $\begin{array}{r}100 \\
70 \\
70 \\
100 \\
200\end{array}$ & $\begin{array}{l}\mathrm{L} \\
\mathrm{L} \\
.04 \\
.02 \\
.02\end{array}$ & $\begin{array}{l}-- \\
-- \\
-- \\
--\end{array}$ & $\begin{array}{l}L \\
L \\
L \\
L \\
L\end{array}$ & $\begin{array}{l}-- \\
\ldots \\
-\ldots \\
--\end{array}$ \\
\hline $\begin{array}{l}77-5 \\
78-5 \\
79-5 \\
80-5 \\
81-5\end{array}$ & $\begin{array}{r}L \\
10 \\
10 \\
L \\
L\end{array}$ & $\begin{array}{r}20 \\
20 \\
20 \\
20 \\
5\end{array}$ & $\begin{array}{r}10 \\
20 \\
L \\
L \\
10\end{array}$ & $\begin{array}{r}10 \\
7 \\
7 \\
7 \\
7\end{array}$ & $\begin{array}{r}700 \\
1,000 \\
700 \\
1,000 \\
300\end{array}$ & $\begin{array}{l}50 \\
70 \\
70 \\
70 \\
50\end{array}$ & $\begin{array}{l}N \\
N \\
N \\
N \\
N\end{array}$ & $\begin{array}{l}7 \\
5 \\
7 \\
5 \\
7\end{array}$ & $\begin{array}{l}N \\
N \\
N \\
N \\
N\end{array}$ & $\begin{array}{r}100 \\
100 \\
100 \\
100 \\
50\end{array}$ & $\begin{array}{l}\mathrm{L}^{.02} \\
.02 \\
.04\end{array}$ & $\begin{array}{l}-- \\
-- \\
-- \\
--\end{array}$ & $\begin{array}{l}L \\
L \\
L \\
L \\
L\end{array}$ & $\begin{array}{l}--- \\
-- \\
-- \\
--- \\
-\end{array}$ \\
\hline $\begin{array}{l}82-S \\
83-R \\
84-S \\
85-S \\
86-S\end{array}$ & $\begin{array}{r}L \\
10 \\
L \\
L \\
L\end{array}$ & $\begin{array}{r}2 \\
2 \\
3 \\
2 \\
10\end{array}$ & $\begin{array}{r}10 \\
N \\
10 \\
10 \\
15\end{array}$ & $\begin{array}{l}L \\
5 \\
L \\
L \\
7\end{array}$ & $\begin{array}{l}300 \\
300 \\
300 \\
300 \\
300\end{array}$ & $\begin{array}{l}20 \\
20 \\
15 \\
15 \\
50\end{array}$ & $\begin{array}{l}\mathbf{N} \\
\mathbf{N} \\
\mathbf{N} \\
\mathbf{N} \\
\mathrm{N}\end{array}$ & $\begin{array}{l}7 \\
7 \\
5 \\
5 \\
5\end{array}$ & $\begin{array}{l}N \\
N \\
N \\
N \\
N\end{array}$ & $\begin{array}{r}100 \\
100 \\
50 \\
70 \\
100\end{array}$ & $\begin{array}{l}.02 \\
.02 \\
.02 \\
.02 \\
.04\end{array}$ & $\begin{array}{l}-- \\
-- \\
-- \\
--\end{array}$ & $\begin{array}{l}L \\
L \\
L \\
L \\
L\end{array}$ & $\begin{array}{r}-- \\
\text { Grn, porphyritic. } \\
-- \\
--\end{array}$ \\
\hline $\begin{array}{l}87-5 \\
88-5 \\
89-5 \\
90-5 \\
91-V\end{array}$ & $\begin{array}{l}L \\
L \\
L \\
L \\
N\end{array}$ & $\begin{array}{l}5 \\
3 \\
3 \\
2 \\
2\end{array}$ & $\begin{array}{r}10 \\
15 \\
20 \\
N \\
N\end{array}$ & $\begin{array}{l}7 \\
5 \\
7 \\
L \\
N\end{array}$ & $\begin{array}{r}500 \\
300 \\
300 \\
300 \\
N\end{array}$ & $\begin{array}{r}50 \\
30 \\
70 \\
20 \\
\text { L }\end{array}$ & $\begin{array}{l}\mathrm{N} \\
\mathrm{N} \\
\mathrm{N} \\
\mathrm{N} \\
\mathrm{N}\end{array}$ & $\begin{array}{r}7 \\
5 \\
15 \\
7 \\
N\end{array}$ & $\begin{array}{l}\mathbf{N} \\
\mathbf{N} \\
\mathbf{N} \\
\mathbf{N} \\
\mathbf{N}\end{array}$ & $\begin{array}{r}50 \\
30 \\
150 \\
20 \\
L\end{array}$ & $\mathrm{~L}^{.02}$ & $\begin{array}{l}-- \\
-- \\
-- \\
--\end{array}$ & $\begin{array}{l}L \\
L \\
L \\
L \\
L\end{array}$ & $\begin{array}{l}-\cdots \\
-- \\
\cdots \\
-\end{array}$ \\
\hline $\begin{array}{l}92-5 \\
93-5 \\
94-5 \\
95-5 \\
96-5\end{array}$ & $\begin{array}{l}N \\
L \\
N \\
N \\
L\end{array}$ & $\begin{array}{r}30 \\
30 \\
2 \\
3 \\
\mathrm{~L}\end{array}$ & $\begin{array}{l}10 \\
20 \\
10 \\
\mathrm{~L} \\
\mathrm{~N}\end{array}$ & $\begin{array}{l}7 \\
7 \\
L \\
L \\
L\end{array}$ & $\begin{array}{l}700 \\
500 \\
300 \\
300 \\
300\end{array}$ & $\begin{array}{l}50 \\
70 \\
15 \\
30 \\
20\end{array}$ & $\begin{array}{l}N \\
N \\
N \\
N \\
N\end{array}$ & $\begin{array}{l}\mathrm{L} \\
5 \\
\mathrm{~L} \\
\mathrm{~L} \\
5\end{array}$ & $\begin{array}{l}\mathbf{N} \\
\mathbf{N} \\
\mathbf{N} \\
\mathbf{N} \\
\mathbf{N}\end{array}$ & $\begin{array}{r}70 \\
70 \\
30 \\
50 \\
100\end{array}$ & $\begin{array}{l}L^{.04} \\
L \\
L\end{array}$ & $\begin{array}{l}-- \\
-- \\
-- \\
--\end{array}$ & $\begin{array}{l}L \\
L \\
L \\
L \\
L\end{array}$ & $\begin{array}{l}--- \\
\cdots \\
\cdots- \\
---\end{array}$ \\
\hline $\begin{array}{l}97-C \\
98-S \\
99-R \\
100-S \\
101-S\end{array}$ & $\begin{array}{r}\mathrm{L} \\
10 \\
10 \\
L \\
15\end{array}$ & $\begin{array}{r}50 \\
2 \\
2 \\
3 \\
7\end{array}$ & $\begin{array}{r}10 \\
15 \\
15 \\
10 \\
\mathrm{~N}\end{array}$ & $\begin{array}{r}15 \\
L \\
L \\
L \\
10\end{array}$ & $\begin{array}{l}700 \\
300 \\
500 \\
500 \\
300\end{array}$ & $\begin{array}{r}150 \\
50 \\
50 \\
30 \\
70\end{array}$ & $\begin{array}{l}{ }^{5} L(20) \\
5_{30} \\
{ }^{5} \mathrm{~L}(20) \\
\mathrm{N} \\
\mathrm{N}\end{array}$ & $\begin{array}{r}15 \\
5 \\
5 \\
15 \\
15\end{array}$ & $\begin{array}{r}250 \\
289 \\
241 \\
N \\
N\end{array}$ & $\begin{array}{r}500 \\
70 \\
70 \\
50 \\
150\end{array}$ & $\begin{array}{l}\mathrm{L} \\
\mathrm{L} \\
\mathrm{L}^{.04}\end{array}$ & $\begin{array}{r}0.52 \\
.24 \\
.20 \\
-- \\
--\end{array}$ & $\begin{array}{r}L \\
20 \\
L \\
L \\
L\end{array}$ & $\begin{array}{l}\text { Stream sediment. } \\
\text { Grn, porphyritic. } \\
-\end{array}$ \\
\hline $\begin{array}{l}102-5 \\
103-5 \\
104-5 \\
105-5 \\
106-5\end{array}$ & $\begin{array}{c}N \\
10 \\
L \\
L \\
L\end{array}$ & $\begin{array}{l}2 \\
L \\
L \\
L \\
L\end{array}$ & $\begin{array}{l}L \\
N \\
N \\
L \\
N\end{array}$ & $\begin{array}{l}L \\
L \\
L \\
5 \\
L\end{array}$ & $\begin{array}{l}300 \\
200 \\
300 \\
300 \\
200\end{array}$ & $\begin{array}{l}15 \\
20 \\
20 \\
30 \\
20\end{array}$ & $\begin{array}{l}\mathrm{N} \\
\mathrm{N} \\
\mathrm{N} \\
\mathrm{N} \\
\mathrm{N}\end{array}$ & $\begin{array}{l}5 \\
7 \\
7 \\
5 \\
7\end{array}$ & $\begin{array}{l}\mathrm{N} \\
\mathrm{N} \\
\mathrm{N} \\
\mathrm{N} \\
\mathrm{N}\end{array}$ & $\begin{array}{r}70 \\
70 \\
70 \\
70 \\
150\end{array}$ & $\begin{array}{l}L \\
L \\
L \\
L \\
L\end{array}$ & $\begin{array}{l}-- \\
-- \\
--\end{array}$ & $\begin{array}{l}L \\
L \\
L \\
L \\
L\end{array}$ & $\begin{array}{l}\cdots \\
\cdots \\
\cdots- \\
\cdots\end{array}$ \\
\hline
\end{tabular}


TABLE 4.-Analyses of samples from the Emigrant

\begin{tabular}{|c|c|c|c|c|c|c|c|c|c|c|c|c|c|c|}
\hline \multirow{2}{*}{$\begin{array}{l}\text { Sample } \\
\text { and } \\
\text { type }\end{array}$} & \multicolumn{4}{|c|}{$\begin{array}{c}\text { Semiquantitative spectrographic } \\
\text { analyses (percent) }\end{array}$} & \multicolumn{10}{|c|}{$\begin{array}{c}\text { Semiquant itat ive spectrographic } \\
\text { analyses }(\mathrm{ppm})^{1}\end{array}$} \\
\hline & $\begin{array}{c}\mathrm{Fe} \\
(0.05)\end{array}$ & $\begin{array}{c}\mathrm{Mg} \\
(0.005)\end{array}$ & $\begin{array}{c}\mathrm{Ca} \\
(0.05)\end{array}$ & $\begin{array}{c}\mathrm{Ti} \\
(0.001)\end{array}$ & $\begin{array}{c}M n \\
(20)\end{array}$ & $\begin{array}{l}\mathrm{Ag} \\
(0.5)\end{array}$ & $\stackrel{B}{(10)}$ & $\begin{array}{c}B a \\
(20)\end{array}$ & $\begin{array}{l}B e \\
\text { (1) }\end{array}$ & $\begin{array}{l}\text { Co } \\
(5)\end{array}$ & $\begin{array}{l}c r \\
(5)\end{array}$ & $\begin{array}{l}\mathrm{Cu} \\
(2)\end{array}$ & $\begin{array}{l}\text { La } \\
(20)\end{array}$ & $\begin{array}{l}\text { Mo } \\
\text { (5) }\end{array}$ \\
\hline $107-5$ & 1.5 & 0.3 & 1.5 & 0.15 & 300 & $\mathrm{~N}$ & $\mathrm{~N}$ & 1,000 & 1.5 & $L$ & $\mathrm{~L}$ & 7 & 20 & $\mathrm{~N}$ \\
\hline $108-5$ & 1.5 & .3 & 1 & .15 & 200 & $\mathrm{~N}$ & N & 700 & L & 7 & L & 7 & L & $\mathrm{N}$ \\
\hline $109-5$ & 2 & .7 & 1.5 & .2 & 300 & $\mathrm{~N}$ & N & 700 & 1 & 7 & 10 & 10 & $L$ & $\mathrm{~N}$ \\
\hline $110-5$ & 2 & .5 & 1.5 & .2 & 200 & N & L & 700 & $i$ & 7 & 7 & 15 & $\mathrm{~N}$ & N \\
\hline $111-s$ & 1.5 & .5 & 1.5 & .3 & 300 & N & N & 700 & $i$ & 7 & 7 & 15 & $\ddot{L}$ & N \\
\hline $112-5$ & 1.5 & .3 & 1 & .3 & 200 & N & N & 1,000 & $L$ & $\mathrm{~N}$ & L & 10 & 20 & $\mathrm{~N}$ \\
\hline $113-5$ & 1.5 & .2 & 1 & .2 & 150 & $N$ & $N$ & 300 & $L$ & $L$ & L & 15 & $L$ & $N$ \\
\hline $114-5$ & 1.5 & .3 & 1 & .2 & 300 & $\mathrm{~N}$ & N & 300 & L & 7 & L & 10 & L & $N$ \\
\hline $115-5$ & 1.5 & .3 & 1 & .2 & 200 & $\mathrm{~N}$ & $\mathrm{~N}$ & 1,000 & $i$ & 5 & L & 7 & $\mathrm{~N}$ & $N$ \\
\hline $116-5$ & 1 & .2 & 1 & .1 & 150 & N & N & 1,000 & L & 5 & $i$ & 7 & $\mathrm{~N}$ & $\mathrm{~N}$ \\
\hline $117-5$ & 1 & .15 & .7 & .15 & 200 & $\mathrm{~N}$ & $\mathrm{~N}$ & 1,000 & L & $N$ & $N$ & 7 & $\mathrm{~N}$ & $\mathrm{~N}$ \\
\hline $118-5$ & 1.5 & .3 & 1 & .3 & 150 & $\mathrm{~N}$ & $\mathrm{~N}$ & 700 & L & 5 & L & 10 & $\mathrm{~N}$ & $\mathrm{~N}$ \\
\hline $119-5$ & 1.5 & .2 & 1.5 & .15 & 150 & N & $\mathrm{N}$ & 700 & 1 & L & L & 10 & $L$ & $N$ \\
\hline $120-5$ & 1.5 & .5 & 1.5 & .15 & 300 & $\mathrm{~N}$ & $\mathrm{~N}$ & 1,000 & L & 7 & 5 & 7 & $\mathrm{~N}$ & $\mathrm{~N}$ \\
\hline $121-5$ & 1.5 & .5 & 1.5 & .3 & 300 & $\mathrm{~N}$ & N & 500 & L & 7 & $L$ & 15 & $\mathrm{~N}$ & $\mathrm{~N}$ \\
\hline $122-S$ & 10 & .3 & 1.5 & .5 & 300 & $\mathrm{~N}$ & $\mathrm{~N}$ & 500 & L & 10 & 30 & 20 & 30 & $\mathrm{~N}$ \\
\hline $123-5$ & 1.5 & .3 & 1.5 & .15 & 200 & $\mathrm{~N}$ & i & 700 & $i$ & $i$ & $\mathrm{~L}$ & 10 & $\mathrm{~N}$ & $\mathrm{~N}$ \\
\hline $124-5$ & 2 & .5 & 1.5 & .2 & 300 & N & $\mathrm{N}$ & 700 & 1 & 7 & 5 & 15 & $\mathrm{~N}$ & $N$ \\
\hline $125-5$ & 1.5 & .5 & 1.5 & .3 & 200 & N & $\mathrm{N}$ & 500 & L & 7 & 10 & 15 & 20 & $\mathrm{~N}$ \\
\hline $126-5$ & 3 & 1 & 2 & 3 & 300 & N & $\mathrm{N}$ & 1,000 & $i$ & 10 & 100 & 15 & $\mathrm{~N}$ & $\mathrm{~N}$ \\
\hline $127-5$ & 3 & 1 & 2 & .5 & 300 & $\mathrm{~N}$ & 10 & 1,500 & $\mathrm{~L}$ & 10 & 50 & 30 & L & N \\
\hline $128-5$ & 3 & .7 & 1.5 & 3 & 300 & $\mathrm{~N}$ & $\mathrm{~L}$ & 1,000 & L & 10 & 70 & 15 & $\mathrm{~N}$ & $\mathrm{~N}$ \\
\hline $129-5$ & 3 & .7 & 1.5 & .3 & 300 & N & $\mathrm{N}$ & 700 & L & 10 & 30 & 15 & $N$ & $N$ \\
\hline $130-5$ & 3 & 1.5 & 1.5 & .3 & 300 & N & $\mathrm{N}$ & 700 & L & 30 & 500 & 30 & $N$ & N \\
\hline $131-A$ & 5 & 2 & 7 & .7 & 500 & N & 10 & 2,000 & 1 & 30 & 50 & 50 & 30 & $\mathrm{~N}$ \\
\hline $132-5$ & 3 & 1.5 & 2 & .5 & 300 & $\mathrm{~N}$ & $\mathrm{~N}$ & 1,500 & L & 30 & 150 & 30 & $L$ & $\mathrm{~N}$ \\
\hline $133-5$ & 3 & 1.5 & 2 & .3 & 300 & $\mathrm{~N}$ & $N$ & 1,000 & L & 15 & 100 & 20 & L & N \\
\hline $134-5$ & 3 & 1.5 & 3 & .3 & 300 & N & N & 1,000 & $\bar{L}$ & 15 & 100 & 20 & L & $N$ \\
\hline $135-5$ & i & .2 & i & .15 & 100 & $\mathrm{~N}$ & $\mathrm{~N}$ & 500 & L & $\mathrm{L}$ & L & 2 & $\mathrm{~N}$ & $N$ \\
\hline $136-5$ & 2 & .3 & 1.5 & .2 & 200 & N & N & 700 & L & 7 & 20 & 10 & $\mathrm{~N}$ & $\mathrm{~N}$ \\
\hline $137-5$ & 1 & .15 & 1 & .1 & 150 & $\mathrm{~N}$ & N & 500 & 1 & $\mathrm{~N}$ & $\mathrm{~N}$ & L & $\mathrm{N}$ & $\mathrm{N}$ \\
\hline $138-5$ & 2 & .7 & 1 & .3 & 300 & N & 10 & 700 & $L$ & 15 & 30 & 20 & $L$ & $N$ \\
\hline $139-5$ & .7 & .2 & $i$ & .07 & 150 & $\mathrm{~N}$ & $\mathrm{~N}$ & 1,000 & $i$ & $L$ & $\mathrm{~N}$ & 7 & 30 & $\mathrm{~N}$ \\
\hline $140-5$ & 3 & .5 & 1.5 & .3 & 200 & $\mathrm{~N}$ & L & 500 & L & 5 & $L$ & 20 & $\mathrm{~N}$ & ${ }^{6} L(2)$ \\
\hline $141-5$ & 7 & 2 & 5 & .5 & 500 & N & 20 & 700 & $\bar{L}$ & 30 & 150 & 30 & N & ${ }^{6} \mathrm{~L}(2)$ \\
\hline $142-5$ & 3 & .7 & 1.5 & .3 & 300 & $\mathrm{~N}$ & L & 300 & L & 10 & L & L & $\mathrm{N}$ & ${ }^{6} L(2)$ \\
\hline $143-V$ & 2 & .05 & .2 & .3 & 70 & 3 & 20 & 500 & L & 7 & 5 & 20 & $\mathrm{~N}$ & ${ }^{6} \mathrm{~L}(2)$ \\
\hline $144-A$ & 3 & .05 & .5 & .03 & 50 & N & $\mathrm{L}$ & $\mathrm{N}$ & $i$ & $N$ & $\hat{L}$ & $L(5$ & 5) $\mathrm{N}$ & 64 \\
\hline $145-5$ & 5 & .7 & 1.5 & .3 & 300 & $\mathrm{~N}$ & 10 & 700 & L & 15 & 30 & 15 & $\mathrm{~L}$ & ${ }^{6} L(2)$ \\
\hline $146-A$ & 7 & 2 & 5 & .5 & 500 & N & $\mathrm{N}$ & 1,500 & $i$ & 30 & 20 & 30 & 20 & ${ }^{6} \mathrm{~L}(2)$ \\
\hline $147-5$ & 5 & 1 & 2 & .5 & 3.00 & . & 510 & 1,000 & $L$ & 20 & 50 & 15 & 30 & 64 \\
\hline $148-v$ & 2 & .02 & .07 & .007 & 15 & 1,500 & 15 & $G$ & L & 15 & $\mathrm{~L}$ & 5,000 & $L$ & ${ }^{6} L(2)$ \\
\hline $149-5$ & 7 & 3 & 3 & .3 & 300 & 10 & 15 & 1,000 & L & 20 & 70 & 30 & 20 & 64 \\
\hline $150-5$ & 5 & 2 & 3 & .3 & 300 & 2 & 20 & 700 & 1.5 & 15 & 30 & 20 & $\mathrm{~L}$ & ${ }^{6} \mathrm{~L}(2)$ \\
\hline $151-5$ & 7 & 2 & 2 & .5 & 300 & 1 & 10 & 700 & 1 & 15 & 50 & 20 & 20 & ${ }^{6} L(2)$ \\
\hline $152-s$ & 5 & 1.5 & 2 & .3 & 200 & & 510 & 700 & L & 15 & 50 & 15 & 20 & ${ }^{6} L(2)$ \\
\hline $153-5$ & 5 & 2 & 2 & .3 & 300 & & 530 & 700 & 15 & 10 & 20 & 10 & 20 & ${ }^{6} \mathrm{~L}(2)$ \\
\hline $154-5$ & 7 & 3 & 3 & .5 & 500 & L & 30 & 700 & 1.5 & 20 & 50 & 20 & $L$ & 64 \\
\hline $155-5$ & 5 & 3 & 3 & .3 & 500 & . & 515 & 700 & $L$ & 20 & 150 & 20 & $\mathrm{~L}$ & ${ }^{6} \mathrm{~L}(2)$ \\
\hline $156-5$ & 7 & 3 & 5 & .3 & 500 & $\mathrm{~N}$ & 20 & 1,000 & $L$ & 20 & 100 & 20 & L & ${ }^{6} 8$ \\
\hline $157-\mathrm{s}$ & 3 & 1.5 & 2 & .3 & 200 & $\mathrm{~N}$ & 10 & 700 & L & 10 & 7 & 10 & 20 & 64 \\
\hline $158-5$ & 5 & 2 & 2 & .3 & 300 & L & 15 & 1,000 & L & 20 & 70 & 15 & $L$ & ${ }^{6} L(2)$ \\
\hline $159-5$ & 3 & i & 2 & .3 & 300 & $\mathrm{~N}$ & 10 & 500 & 15 & 15 & 10 & 10 & 20 & ${ }^{6} \mathrm{~L}(2)$ \\
\hline $160-5$ & 1.5 & .3 & 1 & 1.5 & 150 & L & $L$ & 500 & 1 & 5 & L & 10 & $\mathrm{~N}$ & ${ }^{6} \mathrm{~L}(2)$ \\
\hline $161-5$ & 5 & 1.5 & 3 & .3 & 300 & $\mathrm{~N}$ & 10 & 700 & 1 & 15 & 100 & 10 & L & ${ }^{6} L(2)$ \\
\hline $162-5$ & 1 & .3 & .7 & .1 & 70 & N & L & 300 & 1 & L & L & $L(5)$ & $\mathrm{N}$ & ${ }^{6} L(2)$ \\
\hline $163-5$ & 2 & .3 & 1.5 & .2 & 200 & N & L & 500 & L & 7 & 5 & $L(5)$ & $N$ & ${ }^{6} \mathrm{~L}(2)$ \\
\hline $164-5$ & 3 & .7 & 1.5 & .3 & 300 & $\mathrm{~N}$ & 10 & 700 & L & 10 & 5 & 5 & L & 64 \\
\hline $165-5$ & 3 & .7 & 1.5 & .3 & 500 & $\mathrm{~N}$ & $\mathrm{~L}$ & 500 & L & 10 & 15 & 5 & L & ${ }^{6} L(2)$ \\
\hline $166-5$ & 3 & .7 & 1.5 & .3 & 500 & N & 10 & 700 & L & 10 & 10 & 5 & $\mathrm{~N}$ & ${ }^{6} \mathrm{~L}(2)$ \\
\hline
\end{tabular}




\begin{tabular}{|c|c|c|c|c|c|c|c|c|c|c|c|c|c|c|}
\hline \multirow{2}{*}{$\begin{array}{c}\text { Sample } \\
\text { and } \\
\text { type }\end{array}$} & \multicolumn{10}{|c|}{$\begin{array}{l}\text { Semiquantitative spectrographic } \\
\text { analyses }(\mathrm{ppm})-\text { Cont inued }\end{array}$} & \multicolumn{3}{|c|}{$\begin{array}{l}\text { Chemical analyses } \\
(\mathrm{ppm})\end{array}$} & \multirow[t]{2}{*}{ Remarks } \\
\hline & $\begin{array}{c}\mathrm{Nb} \\
(10)\end{array}$ & $\stackrel{\mathrm{Ni}}{(2)}$ & $\begin{array}{c}\mathrm{Pb} \\
(10)\end{array}$ & $\begin{array}{l}S c \\
(5)\end{array}$ & $\begin{array}{c}\mathrm{Sr} \\
(50)\end{array}$ & $\begin{array}{l}v \\
(10)\end{array}$ & $\underset{(50)}{W}$ & $\begin{array}{l}Y \\
(5)\end{array}$ & $\underset{(200)}{\mathrm{Zn}}$ & $\underset{(10)}{\mathrm{Zr}}$ & $\begin{array}{c}A u^{2} \\
(0.02)\end{array}$ & $\begin{array}{c}\mathrm{Hg}^{3} \\
(0.01)\end{array}$ & $\begin{array}{l}u^{4} \\
(20)\end{array}$ & \\
\hline
\end{tabular}

\begin{tabular}{|c|c|c|c|c|c|c|c|c|c|c|c|c|c|c|}
\hline $\begin{array}{l}107-5 \\
108-5 \\
109-5 \\
110-5 \\
111-5\end{array}$ & $\begin{array}{c}L \\
L \\
10 \\
L \\
L\end{array}$ & $\begin{array}{l}2 \\
3 \\
5 \\
7 \\
7\end{array}$ & $\begin{array}{r}L \\
15 \\
N \\
10 \\
15\end{array}$ & $\begin{array}{l}5 \\
\mathrm{~L} \\
7 \\
\mathrm{~L} \\
7\end{array}$ & $\begin{array}{l}300 \\
500 \\
500 \\
700 \\
500\end{array}$ & $\begin{array}{l}20 \\
30 \\
50 \\
50 \\
50\end{array}$ & $\begin{array}{l}\mathrm{N} \\
\mathrm{N} \\
\mathrm{N} \\
\mathrm{N} \\
\mathrm{N}\end{array}$ & $\begin{array}{r}7 \\
L \\
15 \\
5 \\
7\end{array}$ & $\begin{array}{l}N \\
N \\
N \\
N \\
N\end{array}$ & $\begin{array}{r}100 \\
30 \\
70 \\
15 \\
30\end{array}$ & $\begin{array}{l}\mathrm{L} \\
\mathrm{L} \\
\mathrm{L} \\
\mathrm{L} \\
0.02\end{array}$ & $\begin{array}{l}\ldots \\
\ldots \\
\ldots \\
\ldots\end{array}$ & $\begin{array}{l}L \\
L \\
L \\
L \\
L\end{array}$ & $\begin{array}{l}--- \\
-- \\
\cdots- \\
\cdots\end{array}$ \\
\hline $\begin{array}{l}112-5 \\
113-5 \\
114-5 \\
115-5 \\
116-5\end{array}$ & $\begin{array}{r}10 \\
N \\
L \\
L \\
L\end{array}$ & $\begin{array}{l}3 \\
3 \\
5 \\
7 \\
5\end{array}$ & $\begin{array}{r}10 \\
10 \\
15 \\
L \\
10\end{array}$ & $\begin{array}{l}L \\
L \\
L \\
L \\
L\end{array}$ & $\begin{array}{l}500 \\
500 \\
500 \\
500 \\
300\end{array}$ & $\begin{array}{l}30 \\
30 \\
30 \\
30 \\
20\end{array}$ & $\begin{array}{l}\mathrm{N} \\
\mathrm{N} \\
\mathrm{N} \\
\mathrm{N} \\
\mathrm{N}\end{array}$ & $\begin{array}{l}7 \\
5 \\
5 \\
L \\
N\end{array}$ & $\begin{array}{l}N \\
N \\
N \\
N \\
N\end{array}$ & $\begin{array}{r}15 \\
100 \\
20 \\
L \\
20\end{array}$ & $\begin{array}{l}\mathrm{L} \\
.02 \\
.02\end{array}$ & $\begin{array}{l}-- \\
-- \\
-- \\
--\end{array}$ & $\begin{array}{l}L \\
L \\
L \\
L \\
L\end{array}$ & $\begin{array}{l}-- \\
--- \\
-\ldots \\
--\end{array}$ \\
\hline $\begin{array}{l}117-5 \\
118-5 \\
119-5 \\
120-5 \\
121-5\end{array}$ & $\begin{array}{l}N \\
10 \\
10 \\
10 \\
10\end{array}$ & $\begin{array}{l}3 \\
5 \\
3 \\
3 \\
5\end{array}$ & $\begin{array}{c}10 \\
10 \\
L \\
L \\
L\end{array}$ & $\begin{array}{l}N \\
L \\
L \\
L \\
5\end{array}$ & $\begin{array}{l}300 \\
300 \\
300 \\
500 \\
500\end{array}$ & $\begin{array}{l}15 \\
30 \\
30 \\
30 \\
50\end{array}$ & $\begin{array}{l}\mathrm{N} \\
\mathrm{N} \\
\mathrm{N} \\
\mathrm{N} \\
\mathrm{N}\end{array}$ & $\begin{array}{l}N \\
L \\
L \\
N \\
5\end{array}$ & $\begin{array}{l}\mathrm{N} \\
\mathrm{N} \\
\mathrm{N} \\
\mathrm{N} \\
\mathrm{N}\end{array}$ & $\begin{array}{r}50 \\
70 \\
50 \\
L \\
20\end{array}$ & $\begin{array}{l}.04 \\
.08 \\
.02\end{array}$ & $\begin{array}{l}-- \\
-- \\
-- \\
--\end{array}$ & $\begin{array}{l}L \\
L \\
L \\
L \\
L\end{array}$ & $\begin{array}{l}--- \\
--- \\
--- \\
--- \\
--\end{array}$ \\
\hline $\begin{array}{l}122-5 \\
123-5 \\
124-5 \\
125-5 \\
126-5\end{array}$ & $\begin{array}{r}15 \\
L \\
L \\
15 \\
10\end{array}$ & $\begin{array}{r}7 \\
3 \\
7 \\
7 \\
20\end{array}$ & $\begin{array}{c}L \\
10 \\
10 \\
L \\
L\end{array}$ & $\begin{array}{l}7 \\
\mathrm{~L} \\
\mathrm{~L} \\
\mathrm{~L} \\
7\end{array}$ & $\begin{array}{r}500 \\
500 \\
700 \\
700 \\
1,000\end{array}$ & $\begin{array}{r}200 \\
20 \\
30 \\
30 \\
50\end{array}$ & $\begin{array}{l}N \\
N \\
N \\
N \\
N\end{array}$ & $\begin{array}{r}15 \\
N \\
N \\
L \\
7\end{array}$ & $\begin{array}{l}\mathrm{N} \\
\mathrm{N} \\
\mathrm{N} \\
\mathrm{N} \\
\mathrm{N}\end{array}$ & $\begin{array}{r}500 \\
10 \\
15 \\
70 \\
70\end{array}$ & $\begin{array}{l}\mathrm{L} \\
\mathrm{L} \\
\mathrm{L} \\
\mathrm{L} \\
.02\end{array}$ & $\begin{array}{l}-- \\
-- \\
-- \\
--\end{array}$ & $\begin{array}{l}L \\
L \\
L \\
L \\
L\end{array}$ & $\begin{array}{l}--- \\
-- \\
-\cdots \\
-- \\
-.\end{array}$ \\
\hline $\begin{array}{l}127-5 \\
128-5 \\
129-5 \\
130-5 \\
131-A\end{array}$ & $\begin{array}{r}10 \\
10 \\
L \\
L \\
L\end{array}$ & $\begin{array}{r}30 \\
30 \\
20 \\
100 \\
30\end{array}$ & $\begin{array}{r}10 \\
L \\
10 \\
L \\
L\end{array}$ & $\begin{array}{r}10 \\
7 \\
7 \\
10 \\
15\end{array}$ & $\begin{array}{r}700 \\
1,000 \\
700 \\
700 \\
1,500\end{array}$ & $\begin{array}{l}70 \\
50 \\
50 \\
70 \\
70\end{array}$ & $\begin{array}{l}\mathrm{N} \\
\mathrm{N} \\
\mathrm{N} \\
\mathrm{N} \\
\mathrm{N}\end{array}$ & $\begin{array}{r}10 \\
7 \\
5 \\
10 \\
15\end{array}$ & $\begin{array}{l}\mathrm{N} \\
\mathrm{N} \\
\mathrm{N} \\
\mathrm{N} \\
\mathrm{N}\end{array}$ & $\begin{array}{r}150 \\
150 \\
70 \\
100 \\
150\end{array}$ & $\begin{array}{l}\mathrm{L} \\
\mathrm{L} \\
\mathrm{L} \\
\mathrm{L} \\
\mathrm{L}\end{array}$ & $\begin{array}{l}-- \\
-- \\
-- \\
\cdots\end{array}$ & $\begin{array}{l}L \\
L \\
L \\
L \\
L\end{array}$ & Vol, iron-stained ash \\
\hline $\begin{array}{l}132-5 \\
133-5 \\
134-5 \\
135-5 \\
136-5\end{array}$ & $\begin{array}{r}10 \\
L \\
10 \\
L \\
L\end{array}$ & $\begin{array}{r}30 \\
30 \\
30 \\
2 \\
7\end{array}$ & $\begin{array}{l}L \\
L \\
L \\
L\end{array}$ & $\begin{array}{r}10 \\
7 \\
10 \\
N \\
L\end{array}$ & $\begin{array}{r}1,500 \\
1,000 \\
700 \\
700 \\
700\end{array}$ & $\begin{array}{l}70 \\
70 \\
70 \\
15 \\
30\end{array}$ & $\begin{array}{l}\mathrm{N} \\
\mathrm{N} \\
\mathrm{N} \\
\mathrm{N} \\
\mathrm{N}\end{array}$ & $\begin{array}{r}10 \\
7 \\
10 \\
N \\
N\end{array}$ & $\begin{array}{l}\mathrm{N} \\
\mathrm{N} \\
\mathrm{N} \\
\mathrm{N} \\
\mathrm{N}\end{array}$ & $\begin{array}{r}200 \\
70 \\
70 \\
15 \\
30\end{array}$ & $\begin{array}{l}L \\
L \\
.04 \\
.02\end{array}$ & $\begin{array}{l}-- \\
-- \\
-- \\
-- \\
-\end{array}$ & $\begin{array}{l}\mathrm{L} \\
\mathrm{L} \\
\mathrm{L} \\
\mathrm{L} \\
\mathrm{L}\end{array}$ & $\begin{array}{l}\cdots \\
\cdots \\
\cdots- \\
\cdots\end{array}$ \\
\hline $\begin{array}{l}137-5 \\
138-5 \\
139-5 \\
140-5 \\
141-5\end{array}$ & $\begin{array}{l}L \\
L \\
N \\
L\end{array}$ & $\begin{array}{r}L \\
10 \\
L \\
5 \\
50\end{array}$ & $\begin{array}{l}\mathrm{L} \\
10 \\
10 \\
30 \\
30\end{array}$ & $\begin{array}{r}L \\
7 \\
L \\
L \\
20\end{array}$ & $\begin{array}{l}300 \\
700 \\
300 \\
500 \\
700\end{array}$ & $\begin{array}{r}15 \\
70 \\
10 \\
50 \\
200\end{array}$ & $\begin{array}{l}\mathrm{N} \\
\mathrm{N} \\
\mathrm{N} \\
\mathrm{N} \\
\mathrm{N}\end{array}$ & $\begin{array}{c}N \\
L \\
L \\
L(10) \\
20\end{array}$ & $\begin{array}{r}N \\
N \\
N \\
246 \\
240\end{array}$ & $\begin{array}{r}20 \\
70 \\
15 \\
100 \\
150\end{array}$ & $\begin{array}{l}\mathrm{L} \\
\mathrm{L} \\
\mathrm{L} \\
\mathrm{L}\end{array}$ & $\begin{array}{l}-. \\
-- \\
-- \\
.28 \\
.18\end{array}$ & $\begin{array}{l}\mathrm{L} \\
\mathrm{L} \\
\mathrm{L} \\
\mathrm{L} \\
\mathrm{L}\end{array}$ & $\begin{array}{ll}B i & =L . \\
B i & =L .\end{array}$ \\
\hline $\begin{array}{l}142-S \\
143-V \\
144-A \\
145-S \\
146-A\end{array}$ & $\begin{array}{l}L \\
L \\
L \\
L \\
10\end{array}$ & $\begin{array}{r}7 \\
5 \\
5 \\
10 \\
20\end{array}$ & $\begin{array}{l}30 \\
30 \\
70 \\
30 \\
30\end{array}$ & $\begin{array}{r}\mathrm{L} \\
\mathrm{N} \\
\mathrm{N} \\
7 \\
30\end{array}$ & $\begin{array}{r}500 \\
100 \\
N \\
700 \\
1,000\end{array}$ & $\begin{array}{r}50 \\
30 \\
L \\
70 \\
100\end{array}$ & $\begin{array}{l}\mathrm{N} \\
\mathrm{N} \\
\mathrm{N} \\
\mathrm{N} \\
\mathrm{N}\end{array}$ & $\begin{array}{l}L(10) \\
L(10) \\
N \\
15 \\
20\end{array}$ & $\begin{array}{r}260 \\
226 \\
2 L(25) \\
262 \\
269\end{array}$ & $\begin{array}{r}70 \\
150 \\
10 \\
100 \\
150\end{array}$ & $\begin{array}{l}L \\
L \\
L \\
L \\
L\end{array}$ & $\begin{array}{l}.12 \\
1.4 \\
.20 \\
.09 \\
.14\end{array}$ & $\begin{array}{l}L \\
L \\
L \\
L \\
L\end{array}$ & $\begin{array}{l}\text { Quartz, Fe oxide. } \\
\text { Grn, red stained. } \\
\text { Vol, chloritized. }\end{array}$ \\
\hline $\begin{array}{l}147-5 \\
148-V \\
149-5 \\
150-5 \\
151-5\end{array}$ & $\begin{array}{r}10 \\
L \\
15 \\
10 \\
10\end{array}$ & $\begin{array}{r}20 \\
5 \\
30 \\
10 \\
20\end{array}$ & $\begin{array}{r}30 \\
6 \\
200 \\
70 \\
50\end{array}$ & $\begin{array}{r}15 \\
N \\
15 \\
10 \\
15\end{array}$ & $\begin{array}{r}700 \\
300 \\
700 \\
1,000 \\
700\end{array}$ & $\begin{array}{r}100 \\
N \\
100 \\
70 \\
70\end{array}$ & $\begin{array}{l}N \\
N \\
N \\
N \\
N\end{array}$ & $\begin{array}{r}20 \\
N \\
20 \\
15 \\
20\end{array}$ & $\begin{array}{r}245 \\
26,200 \\
255 \\
238 \\
250\end{array}$ & $\begin{array}{r}150 \\
N \\
200 \\
150 \\
150\end{array}$ & $\begin{array}{l}L \\
L \\
L\end{array}$ & $>>6 \begin{array}{r}.09 \\
.70 \\
.20 \\
.16\end{array}$ & $\begin{array}{l}\mathrm{L} \\
\mathrm{L} \\
\mathrm{L} \\
\mathrm{L} \\
\mathrm{L}\end{array}$ & $\begin{array}{l}\cdots \\
--- \\
---\end{array}$ \\
\hline $\begin{array}{l}152-5 \\
153-5 \\
154-5 \\
155-5 \\
156-5\end{array}$ & $\begin{array}{r}L \\
10 \\
10 \\
L \\
L\end{array}$ & $\begin{array}{l}15 \\
10 \\
30 \\
50 \\
30\end{array}$ & $\begin{array}{l}50 \\
50 \\
30 \\
30 \\
30\end{array}$ & $\begin{array}{l}10 \\
10 \\
15 \\
20 \\
20\end{array}$ & $\begin{array}{r}700 \\
700 \\
1,000 \\
700 \\
1,000\end{array}$ & $\begin{array}{r}70 \\
70 \\
150 \\
150 \\
150\end{array}$ & $\begin{array}{l}\mathrm{N} \\
\mathrm{N} \\
\mathrm{N} \\
\mathrm{N} \\
\mathrm{N}\end{array}$ & $\begin{array}{l}20 \\
15 \\
30 \\
20 \\
20\end{array}$ & $\begin{array}{c}240 \\
2 \mathrm{~L}(25) \\
270 \\
261 \\
239\end{array}$ & $\begin{array}{l}150 \\
200 \\
200 \\
150 \\
150\end{array}$ & $\begin{array}{l}.02 \\
.02 \\
.02 \\
.02 \\
L\end{array}$ & $\begin{array}{l}.16 \\
.12 \\
.12 \\
.20 \\
.07\end{array}$ & $\begin{array}{l}L \\
L \\
L \\
L \\
L\end{array}$ & $\begin{array}{l}--- \\
--- \\
--- \\
--- \\
---\end{array}$ \\
\hline $\begin{array}{l}157-5 \\
158-5 \\
159-5 \\
160-5 \\
161-5\end{array}$ & $\begin{array}{r}10 \\
L \\
10 \\
L \\
10\end{array}$ & $\begin{array}{r}5 \\
50 \\
7 \\
5 \\
20\end{array}$ & $\begin{array}{l}50 \\
30 \\
30 \\
30 \\
20\end{array}$ & $\begin{array}{r}7 \\
20 \\
10 \\
N \\
15\end{array}$ & $\begin{array}{r}500 \\
500 \\
500 \\
500 \\
1,000\end{array}$ & $\begin{array}{r}70 \\
100 \\
70 \\
30 \\
100\end{array}$ & $\begin{array}{l}\mathrm{N} \\
\mathrm{N} \\
\mathrm{N} \\
\mathrm{N} \\
\mathrm{N}\end{array}$ & $\begin{array}{l}15 \\
15 \\
10 \\
10 \\
15\end{array}$ & $\begin{array}{l}245 \\
233 \\
269 \\
225 \\
237\end{array}$ & $\begin{array}{r}100 \\
150 \\
100 \\
70 \\
150\end{array}$ & $\begin{array}{l}L^{.02} \\
L \\
L \\
L\end{array}$ & $\begin{array}{l}.10 \\
.07 \\
.05 \\
.05 \\
.09\end{array}$ & $\begin{array}{l}L \\
L \\
20 \\
L \\
L\end{array}$ & $\begin{array}{l}-- \\
\cdots \\
--- \\
---\end{array}$ \\
\hline $\begin{array}{l}162-5 \\
163-5 \\
164-5 \\
165-5 \\
166-5\end{array}$ & $\begin{array}{l}\mathrm{L} \\
\mathrm{L} \\
\mathrm{L} \\
\mathrm{L}\end{array}$ & $\begin{array}{r}3 \\
5 \\
7 \\
10 \\
7\end{array}$ & $\begin{array}{l}30 \\
30 \\
50 \\
30 \\
30\end{array}$ & $\begin{array}{l}N \\
L \\
L \\
5 \\
5\end{array}$ & $\begin{array}{l}300 \\
300 \\
500 \\
500 \\
500\end{array}$ & $\begin{array}{l}20 \\
30 \\
30 \\
30 \\
30\end{array}$ & $\begin{array}{l}N \\
N \\
N \\
N \\
N\end{array}$ & $\begin{array}{c}L(10) \\
N \\
10 \\
L \\
L\end{array}$ & $\begin{array}{l}227 \\
225 \\
270 \\
262 \\
235\end{array}$ & $\begin{array}{r}15 \\
30 \\
70 \\
100 \\
70\end{array}$ & ${\stackrel{.02}{L}{ }_{.02}^{.02}}^{L_{0}}$ & $\begin{array}{l}.10 \\
.07 \\
.07 \\
.07 \\
.09\end{array}$ & $\begin{array}{l}\mathrm{L} \\
\mathrm{L} \\
\mathrm{L} \\
\mathrm{L} \\
\mathrm{L}\end{array}$ & $\begin{array}{l}\cdots- \\
--- \\
-\cdots \\
-- \\
--\end{array}$ \\
\hline
\end{tabular}


TABLE 4.-Analyses of samples from the Emigrant

\begin{tabular}{|c|c|c|c|c|c|c|c|c|c|c|c|c|c|c|}
\hline \multirow{2}{*}{$\begin{array}{l}\text { Sample } \\
\text { and } \\
\text { type }\end{array}$} & \multicolumn{4}{|c|}{$\begin{array}{c}\text { Semiquantitative spectrographic } \\
\text { analyses (percent) }\end{array}$} & \multicolumn{10}{|c|}{$\begin{array}{c}\text { Semiquantitative spect rographic } \\
\text { analyses }(\mathrm{ppm})^{1}\end{array}$} \\
\hline & $\begin{array}{c}\mathrm{Fe} \\
(0.05)\end{array}$ & $(0.005)$ & $\begin{array}{c}\mathrm{Ca} \\
(0.05)\end{array}$ & $\begin{array}{c}\mathrm{Ti} \\
(0.001)\end{array}$ & $\begin{array}{c}M n \\
(20)\end{array}$ & $\begin{array}{l}A g \\
(0.5)\end{array}$ & $\stackrel{B}{(10)}$ & $\begin{array}{c}8 a \\
(20)\end{array}$ & $\begin{array}{l}\mathrm{Be} \\
(1)\end{array}$ & $\begin{array}{l}\text { Co } \\
\text { (5) }\end{array}$ & $\begin{array}{l}C r \\
(5)\end{array}$ & $\begin{array}{l}\mathrm{Cu} \\
(2)\end{array}$ & $\begin{array}{l}\text { La } \\
(20)\end{array}$ & $\begin{array}{l}\text { Mo } \\
(5)\end{array}$ \\
\hline $167-5$ & 7 & 1 & 2 & 1 & 700 & N & L & 700 & L & 15 & 20 & $L(5)$ & 50 & ${ }^{6} L(2)$ \\
\hline $168-5$ & 3 & .7 & 1 & .3 & 500 & $\mathrm{~N}$ & L & 300 & L & 5 & L & $L(5)$ & 20 & 64 \\
\hline $169-5$ & 5 & .7 & 1.5 & .3 & 700 & $\mathrm{~N}$ & L & 700 & 1 & 15 & 15 & 5 & 20 & ${ }^{6} L(2)$ \\
\hline $170-c$ & G & .2 & 1.5 & 1 & 700 & N & $\mathrm{N}$ & $L(50)$ & $\mathrm{N}$ & 30 & 70 & 20. & 100 & ${ }^{6} L(2)$ \\
\hline $171-5$ & 2 & .3 & 1 & .2 & 200 & 0.5 & L & 1,000 & L & 7 & L & $L(5)$ & L & ${ }^{6} L \cdot(2)$ \\
\hline $172-5$ & 7 & 1 & 3 & .5 & 700 & N & 30 & 1,000 & $L$ & 20 & 100 & 20 & 20 & ${ }^{6} L(2)$ \\
\hline $173-5$ & 3 & .7 & 1.5 & .3 & 500 & N & 10 & 300 & L & 10 & 10 & 20 & L & ${ }^{6} L(2)$ \\
\hline $174-5$ & 3 & .5 & 1 & .2 & 500 & $\mathrm{~N}$ & 10 & 300 & L & 10 & $L$ & 20 & L & ${ }^{6} L(2)$ \\
\hline $175-5$ & 2 & .7 & 1.5 & .3 & 500 & N & 10 & 300 & L & 10 & 10 & 5 & L & ${ }^{6} L(2)$ \\
\hline $176-5$ & 7 & 2 & 2 & .5 & 1,000 & N & 20 & 700 & $L$ & 15 & 70 & 20 & 20 & ${ }^{6} L(2)$ \\
\hline $177-5$ & 5 & 2 & 1.5 & .5 & 700 & N & 10 & 700 & $L$ & 15 & 50 & 15 & 20 & ${ }^{5} L(2)$ \\
\hline $178-5$ & 7 & 3 & 2 & 1 & 1,000 & N & 20 & 1,000 & L & 20 & 100 & 30 & 20 & ${ }^{6} \mathrm{~L}(2)$ \\
\hline $179-5$ & 5 & 2 & 1.5 & .3 & 1,000 & $\mathrm{~N}$ & 10 & $1,000^{\circ}$ & 1 & 15 & 50 & 30 & L & ${ }^{6} \mathrm{~L}(2)$ \\
\hline $180-5$ & 3 & 1 & 1.5 & .3 & 700 & N & 10 & 700 & L & 10 & 7 & $L(5)$ & L & ${ }^{6} L(2)$ \\
\hline $181-5$ & 7 & 1.5 & 15 & .5 & 700 & N & 15 & 1,000 & $\mathrm{~L}$ & 15 & 70 & 15 & 20 & ${ }^{6} \mathrm{~L}(2)$ \\
\hline $182-5$ & 7 & 1.5 & 1.5 & .5 & 700 & $\mathrm{~N}$ & 10 & 700 & L & 15 & 70 & 15 & 20 & ${ }^{6} \mathrm{~L}(2)$ \\
\hline $182 a-5$ & 7 & 2 & 1.5 & 1 & 1,000 & N & 30 & 700 & 1 & 20 & 150 & 50 & 20 & ${ }^{6} L(2)$ \\
\hline 183-A & 7 & 2 & 10 & .15 & 2,000 & N & 10 & 2,000 & $L$ & 20 & 15 & 30 & L & ${ }^{6} L(2)$ \\
\hline $184-5$ & 5 & 2 & 5 & .3 & 1,500 . & N & 20 & 700 & L & 15 & 30 & 20 & L & ${ }^{6} L(2)$ \\
\hline $185-5$ & 7 & 1.5 & 1.5 & 1 & $1,000^{\circ}$ & N & 20 & 500 & 1 & 30 & 70 & 30 & 20 & ${ }^{6} \mathrm{~L}(2)$ \\
\hline $186-5$ & 7 & 1.5 & 2 & 1 & 1,000 & N & 15 & 1,000 & 1 & 30 & 70 & 30 & 50 & ${ }^{6} L(2)$ \\
\hline $187-5$ & 7 & 2 & 1.5 & 1 & 1,000 & $N$ & 20 & 700 & .3 & 20 & 100 & 50 & 30 & ${ }^{6} L(2)$ \\
\hline 188-s & 3 & 1 & 7 & .2 & 300 & $N(1)$ & $\cdots$ & 2,000 & N & 7 & 5 & 7 & $N(30)$ & $N(3)^{\circ}$ \\
\hline $189-5$ & 7 & 3 & 7 & 1 & 1,000 & $N(1)$ & $\cdots$ & 1,500 & $\mathrm{~N}$ & 15 & 15 & 15 & 30 & $N(3)$ \\
\hline $190-5$ & 5 & 1.5 & 5 & .3 & 700 & $\mathrm{~N}(\mathrm{i})$ & -- & 3,000 & $\mathrm{~N}$ & 10 & 5 & 10 & $N(30)$ & $\mathrm{N}(3)$ \\
\hline $191-5$ & 7 & 2 & 7 & .7 & 700 & $N(1)$ & -- & 1,500 & $\mathrm{~N}$ & 20 & 15 & 15 & 30. & 10 \\
\hline $192-5$ & 5 & 1.5 & 7 & .7 & 300 & $N(1)$ & $-\cdot$ & 1,500 & N & 10 & 15 & 5 & 50 & 5 \\
\hline $193-5$ & 7 & 1.5 & 7 & .5 & 500 & $N(1)$ & $-\cdot$ & 1,500 & N & 15 & 10 & 10 & 30 & 7 \\
\hline $194-5$ & 7 & 2 & 7 & 1.5 & 700 & $N(1)$ & -- & 1,000 & N & 20 & 7 & 10 & 150 & 7 \\
\hline $195-5$ & 5 & 1.5 & 7 & .3 & 500 & $N(1)$ & $-\cdot$ & 1,500 & $\mathrm{~N}$ & 15 & 5 & 5 & $N(30)$ & $N(3)$ \\
\hline $196-5$ & 5 & 1.5 & 7 & .7 & 700 & $N(1)$ & -- & 1,500 & N & 10 & 5 & 5 & 70 & $N(3)$ \\
\hline $197-5$ & 5 & 1 & 7 & .3 & 500 & $N(1)$ & -- & 1,500 & $\mathrm{~N}$ & 10 & 5 & 7 & $N(30)$ & $N(3)$ \\
\hline $198-5$ & 3 & 1.5 & 5 & .3 & 300 & $N(1)$ & -- & 1,500 & $\mathrm{~N}$ & 7 & 3 & 7 & $N(30)$ & 5 \\
\hline $199-5$ & 3 & 1.5 & 3 & .3 & 500 & $\mathrm{~N}(1)$ & -- & 1,500 & N & 7 & 5 & 10 & $N(30)$ & 5 \\
\hline $200-c$ & 3 & .7 & $i$ & .5 & 200 & 2.3 & 10 & 1,000 & L & 20 & 30 & ${ }^{2} 12$ & 30 & ${ }^{5} L(2)$ \\
\hline $201-R$ & 3 & .7 & 1.5 & .3 & 500 & 2.3 & 10 & 2,000 & $\mathrm{~L}$ & 50 & 15 & ${ }^{2} 10$ & 30 & ${ }^{5} L(2)$ \\
\hline $202-5$ & 2 & .7 & .5 & .3 & 200 & 2.3 & 20 & 1,000 & 1 & 50 & 20 & ${ }^{2} L(10)$ & 30 & ${ }^{5} L(2)$ \\
\hline $203-5$ & 2 & .7 & 1 & .2 & 200 & 2.4 & L & 1,000 & $\mathrm{~L}$ & 30 & 50 & 214 & 20 & ${ }^{5} \mathrm{~L}(2)$ \\
\hline $204-5$ & 1 & .2 & 1 & .15 & 150 & ${ }^{2} L(.2)$ & 10 & 700 & 1.5 & 5 & N & ${ }^{2} L(10)$ & L & ${ }_{5}^{5} L(2)$ \\
\hline $205-c$ & 3 & .7 & 2 & .5 & 300 & 2.3 & L & 700 & L & 30 & 200 & ${ }^{2} 10$ & 30 & ${ }^{5} L(2)$ \\
\hline $206-5$ & 2 & .5 & 2 & .3 & 200 & 2.3 & 10 & 1,000 & L & 20 & 50 & ${ }^{2} L(10)$ & 20 & ${ }^{5} L(2)$ \\
\hline $207-5$ & 2 & .5 & 2 & .3 & 300 & 2.5 & 10 & 1,000 & 1 & 20 & 30 & ${ }^{2} L(10)$ & 20 & $5 L(2)$ \\
\hline $208-5$ & 2 & .5 & 1 & .2 & 300 & 2.3 & $L$ & 1,000 & L & 20 & 30 & ${ }^{2} L(10)$ & 20 & ${ }_{5}^{5} L(2)$ \\
\hline $209-5$ & 3 & .7 & 1.5 & .5 & 500 & 2.4 & 10 & 1,500 & 1 & 50 & 30 & 220 & 20 & ${ }_{5}^{5} L(2)$ \\
\hline $210-5$ & 3 & .7 & 1.5 & .5 & 500 & 2.3 & 10 & 1,500 & 1 & 70 & 50 & ${ }^{2} 13$ & 20 & ${ }^{5} L(2)$ \\
\hline $211-5$ & 3 & .7 & 1.5 & .5 & 500 & 2.4 & 10 & 1,500 & 1 & 50 & 70 & 220 & 20 & ${ }^{5} L(2)$ \\
\hline $212-5$ & 3 & 1 & 1 & .5 & 700 & 2.4 & 15 & 1,000 & 1 & 50 & 50 & 222 & 20 & ${ }^{5} L(2)$ \\
\hline $213-5$ & 3 & .7 & 1.5 & .5 & 500 & 2.4 & 10 & 1,000 & 1 & 50 & 30 & 218 & 20 & ${ }_{5}^{5} L(2)$ \\
\hline $214-A$ & 2 & .2 & 1 & .3 & 200 & 2.4 & L & 1,000 & 1 & 10 & N & 215 & 20 & ${ }^{S L}(2)$ \\
\hline $215-A$ & 3 & .5 & 2 & .5 & 300 & 2.3 & 20 & 1,500 & 1 & 30 & 50 & ${ }^{2} L(10)$ & 30 & $5 L(2)$ \\
\hline $216-5$ & 2 & .2 & 1.5 & .3 & 200 & 2.2 & 10 & 1,000 & L & 15 & 20 & ${ }^{2} L(10)$ & 30 & ${ }^{5} L(2)$ \\
\hline $217-5$ & 2 & .5 & 1.5 & .3 & 300 & 2.4 & 10 & 1,000 & 1 & 20 & 50 & ${ }^{2} L(10)$ & 20 & ${ }_{5}^{5} \mathrm{~L}(2)$ \\
\hline $218-5$ & 3 & .3 & 1.5 & .5 & 300 & 2.3 & 10 & 1,000 & L & 20 & 50. & ${ }^{2} 13$ & 30 & ${ }^{5} L(2)$ \\
\hline $219-5$ & 3 & .5 & 2 & .5 & 500 & 2.3 & 15 & 1,000 & 1 & 20 & 50 & .217 & 30 & $5_{L}(2)$ \\
\hline $220-5$ & 2 & .3 & 1.5 & .3 & 300 & 2.2 & 10 & 700 & 1 & 10 & 20 & ${ }^{2} L(10)$ & 30 & ${ }^{5} L(2)$ \\
\hline $221-5$ & 3 & .5 & 1.5 & .3 & 300 & 2.4 & 10 & 1,000 & 1 & 15 & 50 & ${ }^{2} L(10)$ & 20 & $5 L(2)$ \\
\hline $222-S$ & 1 & .3 & 1 & .15 & 200 & 2. & L & 700 & 1 & 10 & $\mathrm{~N}$ & ${ }^{2} L(10)$ & $\mathrm{N}$ & $5<(2)$ \\
\hline $223-5$ & 3 & 1 & 1.5 & .3 & 1,000 & 2.4 & 10 & 1,500 & 1 & 50 & 100 & 217 & 30 & ${ }^{5} 5(2)$ \\
\hline $224-5$ & 2 & 1 & 1.5 & .5 & 1,500 & 2.3 & L & 1,500 & 1 & 30 & 50 & ${ }^{2} L(10)$ & 20 & ${ }^{5} \mathrm{~L}(2)$ \\
\hline $225-5$ & 1.5 & .3 & 1 & .3 & 200 & 2.3 & L & 700 & 1 & 5 & 10 & $2 L(10)$ & L & ${ }^{5} L(2)$ \\
\hline
\end{tabular}




\section{Basin primitive area, California-Continued}

\begin{tabular}{|c|c|c|c|c|c|c|c|c|c|c|c|c|}
\hline \multirow{2}{*}{$\begin{array}{l}\text { Sample } \\
\text { and } \\
\text { type }\end{array}$} & \multicolumn{10}{|c|}{$\begin{array}{l}\text { Semiquanti tative spectrographic } \\
\text { analyses }(\mathrm{ppm}) \text {--Continued }\end{array}$} & $\begin{array}{c}\text { Chemical analyses } \\
(\mathrm{ppm})\end{array}$ & \multirow{2}{*}{ Remarks } \\
\hline & $\begin{array}{l}\mathrm{Nb} \\
(10)\end{array}$ & $\underset{(2)}{N i}$ & $\begin{array}{l}\mathrm{Pb} \\
(10)\end{array}$ & $\begin{array}{l}S c \\
(5)\end{array}$ & $\begin{array}{l}\mathrm{Sr} \\
(50)\end{array}$ & $\begin{array}{l}v \\
(10)\end{array}$ & $\begin{array}{l}W \\
(50)\end{array}$ & $\begin{array}{c}Y \\
(5)\end{array}$ & $\begin{array}{l}\mathrm{Zn} \\
(200)\end{array}$ & $\begin{array}{l}\mathrm{Zr} \\
(10)\end{array}$ & 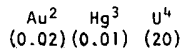 & \\
\hline
\end{tabular}

\begin{tabular}{|c|c|c|c|c|c|c|c|c|c|c|c|c|c|c|}
\hline $\begin{array}{l}167-5 \\
168-5 \\
169-5 \\
170-c \\
171-5\end{array}$ & $\begin{array}{r}10 \\
L \\
L \\
30 \\
L\end{array}$ & $\begin{array}{r}10 \\
5 \\
10 \\
15 \\
5\end{array}$ & $\begin{array}{l}30 \\
30 \\
30 \\
20 \\
30\end{array}$ & $\begin{array}{l}7 \\
5 \\
5 \\
7 \\
1\end{array}$ & $\begin{array}{l}700 \\
300 \\
500 \\
N \\
500\end{array}$ & $\begin{array}{r}50 \\
30 \\
50 \\
300 \\
20\end{array}$ & $\begin{array}{l}\mathrm{N} \\
\mathrm{N} \\
\mathrm{N} \\
\mathrm{N} \\
\mathrm{N}\end{array}$ & $\begin{array}{r}15 \\
10 \\
10 \\
50 \\
5\end{array}$ & $\begin{array}{l}251 \\
252 \\
251 \\
246 \\
244\end{array}$ & $\begin{array}{r}150 \\
70 \\
150 \\
500 \\
70\end{array}$ & $\begin{array}{l}L \\
L \\
0.02 \\
L \\
L\end{array}$ & $\begin{array}{l}0.04 \\
.05 \\
.04 \\
.28 \\
.05\end{array}$ & $\begin{array}{l}\mathrm{L} \\
\mathrm{L} \\
\mathrm{L} \\
\mathrm{L} \\
\mathrm{L}\end{array}$ & Fe, $G=20$ percent \\
\hline $\begin{array}{l}172-5 \\
173-5 \\
174-5 \\
175-5 \\
176-5\end{array}$ & $\begin{array}{l}\mathrm{L} \\
\mathrm{L} \\
\mathrm{L} \\
10\end{array}$ & $\begin{array}{r}30 \\
7 \\
5 \\
7 \\
20\end{array}$ & $\begin{array}{l}30 \\
50 \\
50 \\
50 \\
30\end{array}$ & $\begin{array}{r}20 \\
5 \\
L \\
L \\
15\end{array}$ & $\begin{array}{l}700 \\
500 \\
500 \\
500 \\
700\end{array}$ & $\begin{array}{r}100 \\
20 \\
. \quad 20 \\
20 \\
50\end{array}$ & $\begin{array}{l}\mathrm{N} \\
\mathrm{N} \\
\mathrm{N} \\
\mathrm{N} \\
\mathrm{N}\end{array}$ & $\begin{array}{r}20 \\
\mathrm{~L} \\
\mathrm{~L} \\
7 \\
15\end{array}$ & $\begin{array}{l}264 \\
235 \\
252 \\
261 \\
264\end{array}$ & $\begin{array}{r}200 \\
70 \\
70 \\
70 \\
300\end{array}$ & $\begin{array}{l}L \\
L \\
L^{.03}\end{array}$ & $\begin{array}{l}.07 \\
.05 \\
.03 \\
.05 \\
.05\end{array}$ & $\begin{array}{l}\mathrm{L} \\
\mathrm{L} \\
\mathrm{L} \\
\mathrm{L} \\
\mathrm{L}\end{array}$ & $\begin{array}{l}--- \\
--- \\
--- \\
---\end{array}$ \\
\hline $\begin{array}{l}177-50 \\
178-5 \\
179-5 \\
180-5 \\
181-5\end{array}$ & $\begin{array}{r}\mathrm{L} \\
10 \\
\mathrm{~L} \\
\mathrm{~L} \\
\mathrm{~L}\end{array}$ & $\begin{array}{r}20 \\
30 \\
15 \\
7 \\
20\end{array}$ & $\begin{array}{l}30 \\
30 \\
30 \\
30 \\
20\end{array}$ & $\begin{array}{r}15 \\
20 \\
10 \\
5 \\
10\end{array}$ & $\begin{array}{l}500 \\
700 \\
700 \\
700 \\
700\end{array}$ & $\begin{array}{r}50 \\
100 \\
100 \\
30 \\
50\end{array}$ & $\begin{array}{l}\mathrm{N} \\
\mathrm{N} \\
\mathrm{N} \\
\mathrm{N} \\
\mathrm{N}\end{array}$ & $\begin{array}{l}15 \\
15 \\
15 \\
10 \\
15\end{array}$ & $\begin{array}{r}247 \\
296 \\
2110 \\
274 \\
276\end{array}$ & $\begin{array}{l}150 \\
200 \\
150 \\
150 \\
150\end{array}$ & $L^{L} L^{.03}$ & $\begin{array}{l}.07 \\
.09 \\
.09 \\
.05 \\
.07\end{array}$ & $\begin{array}{l}\mathrm{L} \\
\mathrm{L} \\
\mathrm{L} \\
\mathrm{L} \\
\mathrm{L}\end{array}$ & $\begin{array}{c}\text { Debris, cirque basin. } \\
- \\
-- \\
- \\
--\end{array}$ \\
\hline $\begin{array}{l}182-5 \\
182 a-5 \\
183-V \\
184-5 \\
185-5\end{array}$ & $\begin{array}{r}\mathrm{L} \\
10 \\
\mathrm{~L} \\
\mathrm{~L} \\
\mathrm{~L}\end{array}$ & $\begin{array}{l}20 \\
70 \\
50 \\
20 \\
70\end{array}$ & $\begin{array}{l}20 \\
30 \\
30 \\
30 \\
20\end{array}$ & $\begin{array}{l}10 \\
30 \\
7 \\
15 \\
30\end{array}$ & $\begin{array}{r}500 \\
500 \\
1,000 \\
700 \\
700\end{array}$ & $\begin{array}{r}50 \\
70 \\
30 \\
50 \\
200\end{array}$ & $\begin{array}{l}\mathrm{N} \\
\mathrm{N} \\
\mathrm{N} \\
\mathrm{N} \\
\mathrm{N}\end{array}$ & $\begin{array}{r}15 \\
20 \\
L \\
15 \\
30\end{array}$ & $\begin{array}{r}280 \\
269 \\
2140 \\
278 \\
274\end{array}$ & $\begin{array}{r}200 \\
300 \\
30 \\
150 \\
150\end{array}$ & $\begin{array}{l}L \\
L \\
L \\
L \\
L\end{array}$ & $\begin{array}{l}.05 \\
.10 \\
.12 \\
.05 \\
.14\end{array}$ & $\begin{array}{l}\mathrm{L} \\
\mathrm{L} \\
\mathrm{L} \\
\mathrm{L} \\
\mathrm{L}\end{array}$ & Pyrite, malachite, barite. \\
\hline $\begin{array}{l}186-5 \\
187-5 \\
188-5 \\
189-5 \\
190-5\end{array}$ & $\begin{array}{r}20 \\
20 \\
N \\
20 \\
10\end{array}$ & $\begin{array}{c}70 \\
70 \\
L(3) \\
7 \\
L(3)\end{array}$ & $\begin{array}{l}30 \\
50 \\
50 \\
30 \\
30\end{array}$ & $\begin{array}{l}30 \\
30 \\
-- \\
--\end{array}$ & $\begin{array}{l}700 \\
500 \\
700 \\
500 \\
700\end{array}$ & $\begin{array}{r}200 \\
200 \\
50 \\
100 \\
70\end{array}$ & $\begin{array}{c}N \\
N \\
N(100) \\
N(100) \\
N(100)\end{array}$ & $\begin{array}{l}30 \\
30 \\
15 \\
50 \\
15\end{array}$ & $\begin{array}{l}265 \\
282 \\
268 \\
286 \\
268\end{array}$ & $\begin{array}{l}300 \\
300 \\
100 \\
100 \\
100\end{array}$ & $\begin{array}{l}L \\
L \\
L \\
L \\
L\end{array}$ & $\begin{array}{l}.09 \\
.07 \\
-- \\
-- \\
--\end{array}$ & $\begin{array}{l}\mathrm{L} \\
\mathrm{L} \\
-- \\
--\end{array}$ & $\begin{array}{c}(T a, T e, P d, \\
P t), N . \\
\text { Do. } \\
\text { Do. }\end{array}$ \\
\hline $\begin{array}{l}191-5 \\
192-5 \\
193-5 \\
194-5 \\
195-5\end{array}$ & $\begin{array}{l}10 \\
10 \\
10 \\
15 \\
10\end{array}$ & $\begin{array}{r}15 \\
10 \\
10 \\
5 \\
7\end{array}$ & $\begin{array}{l}50 \\
30 \\
30 \\
30 \\
30\end{array}$ & $\begin{array}{l}-- \\
-- \\
-- \\
-\end{array}$ & $\begin{array}{l}700 \\
700 \\
700 \\
700 \\
700\end{array}$ & $\begin{array}{r}100 \\
70 \\
50 \\
70 \\
70\end{array}$ & $\begin{array}{l}N(100) \\
N(100) \\
N(100) \\
N(100) \\
N(100)\end{array}$ & $\begin{array}{r}15 \\
30 \\
20 \\
70 \\
L(10)\end{array}$ & $\begin{array}{l}297 \\
242 \\
272 \\
274 \\
266\end{array}$ & $\begin{array}{l}100 \\
150 \\
100 \\
300 \\
15\end{array}$ & $\begin{array}{l}L \\
L \\
L \\
L\end{array}$ & $\begin{array}{l}-- \\
-- \\
-- \\
--\end{array}$ & $\begin{array}{l}-- \\
-- \\
-- \\
--\end{array}$ & $\begin{array}{l}\text { Do. } \\
\text { Do. } \\
\text { Do. } \\
\text { Do. } \\
\text { Do. }\end{array}$ \\
\hline $\begin{array}{l}196-5 \\
197-5 \\
198-5 \\
199-5 \\
200-C\end{array}$ & $\begin{array}{r}10 \\
10 \\
10 \\
10 \\
\mathrm{~L}\end{array}$ & $\begin{array}{r}7 \\
7 \\
3 \\
3 \\
20\end{array}$ & $\begin{array}{r}30 \\
30 \\
20 \\
20 \\
225\end{array}$ & $\begin{array}{l}-- \\
-- \\
-- \\
7\end{array}$ & $\begin{array}{r}700 \\
1,000 \\
700 \\
700 \\
1,000\end{array}$ & $\begin{array}{l}70 \\
50 \\
50 \\
50 \\
30\end{array}$ & $\begin{array}{l}N(100) \\
N(100) \\
N(100) \\
N(100) \\
5 L(20)\end{array}$ & $\begin{array}{l}30 \\
15 \\
15 \\
10 \\
10\end{array}$ & $\begin{array}{l}270 \\
266 \\
251 \\
266 \\
257\end{array}$ & $\begin{array}{r}70 \\
70 \\
50 \\
150 \\
100\end{array}$ & $\begin{array}{l}L \\
L \\
L \\
L\end{array}$ & $\begin{array}{l}-- \\
-- \\
-- \\
-- \\
--\end{array}$ & $\begin{array}{l}-- \\
-- \\
-- \\
L\end{array}$ & $\begin{array}{cl}\text { Do. } & \\
\text { Do. } \\
\text { Do. } \\
\text { Do. } \\
\text { Sand, Grouse Creek. }\end{array}$ \\
\hline $\begin{array}{l}201-R \\
202-S \\
203-S \\
204-S \\
205-C\end{array}$ & $\begin{array}{l}\mathrm{L} \\
\mathrm{L} \\
\mathrm{L} \\
\mathrm{L} \\
\mathrm{L}\end{array}$ & $\begin{array}{r}10 \\
50 \\
30 \\
N \\
50\end{array}$ & $\begin{array}{c}2 L(25) \\
{ }^{2} L(25) \\
225 \\
2 L(25) \\
230\end{array}$ & $\begin{array}{r}7 \\
7 \\
7 \\
N \\
10\end{array}$ & $\begin{array}{r}2,000 \\
700 \\
1,000 \\
700 \\
1,000\end{array}$ & $\begin{array}{l}30 \\
50 \\
50 \\
20 \\
50\end{array}$ & $\begin{array}{l}5 \mathrm{~L}(20) \\
5 \mathrm{~L}(20) \\
5 \mathrm{~L}(20) \\
5 \mathrm{~L}(20) \\
5 \mathrm{~L}(20)\end{array}$ & $\begin{array}{r}15 \\
10 \\
10 \\
5 \\
15\end{array}$ & $\begin{array}{l}250 \\
231 \\
250 \\
227 \\
245\end{array}$ & $\begin{array}{r}100 \\
100 \\
70 \\
20 \\
70\end{array}$ & $\begin{array}{l}L \\
L \\
L \\
L \\
L\end{array}$ & $\begin{array}{l}-- \\
-- \\
-- \\
-- \\
--\end{array}$ & $\begin{array}{l}\mathrm{L} \\
\mathrm{L} \\
\mathrm{L} \\
\mathrm{L} \\
\mathrm{L}\end{array}$ & Sand, Summit Creek. \\
\hline $\begin{array}{l}206-5 \\
207-5 \\
208-5 \\
209-5 \\
210-5\end{array}$ & $\begin{array}{r}\mathbf{L} \\
10 \\
\mathbf{L} \\
10 \\
10\end{array}$ & $\begin{array}{l}30 \\
30 \\
20 \\
50 \\
50\end{array}$ & $\begin{array}{l}233 \\
230 \\
232 \\
235 \\
232\end{array}$ & $\begin{array}{r}7 \\
7 \\
5 \\
7 \\
10\end{array}$ & $\begin{array}{l}1,000 \\
1,000 \\
1,000 \\
1,000 \\
1,000\end{array}$ & $\begin{array}{l}50 \\
50 \\
50 \\
70 \\
70\end{array}$ & $\begin{array}{l}5_{\mathrm{L}}(20) \\
5_{\mathrm{L}}(20) \\
5_{\mathrm{L}}(20) \\
5_{\mathrm{L}}(20) \\
5_{\mathrm{L}}(20)\end{array}$ & $\begin{array}{l}10 \\
10 \\
10 \\
10 \\
15\end{array}$ & $\begin{array}{l}237 \\
240 \\
250 \\
273 \\
247\end{array}$ & $\begin{array}{r}50 \\
100 \\
100 \\
200 \\
200\end{array}$ & $\begin{array}{l}\mathrm{L} \\
\mathrm{L} \\
\mathrm{L} \\
\mathrm{L} \\
\mathrm{L}\end{array}$ & $\begin{array}{l}-- \\
-- \\
-- \\
-- \\
--\end{array}$ & $\begin{array}{l}\mathrm{L} \\
\mathrm{L} \\
\mathrm{L} \\
\mathrm{L} \\
\mathrm{L}\end{array}$ & $\begin{array}{l}-\ldots \\
\ldots- \\
-\ldots \\
---\end{array}$ \\
\hline $\begin{array}{l}211-5 \\
212-S \\
213-S \\
214-A \\
215-A\end{array}$ & $\begin{array}{l}10 \\
10 \\
10 \\
10 \\
10\end{array}$ & $\begin{array}{r}70 \\
50 \\
30 \\
L \\
30\end{array}$ & $\begin{array}{l}232 \\
236 \\
236 \\
235 \\
233\end{array}$ & $\begin{array}{r}10 \\
10 \\
7 \\
5 \\
10\end{array}$ & $\begin{array}{r}1,500 \\
700 \\
1,000 \\
1,000 \\
1,500\end{array}$ & $\begin{array}{l}50 \\
70 \\
70 \\
50 \\
70\end{array}$ & $\begin{array}{l}5 L(20) \\
5 L(20) \\
5 L(20) \\
5 L(20) \\
5 L(20)\end{array}$ & $\begin{array}{r}15 \\
15 \\
10 \\
5 \\
15\end{array}$ & $\begin{array}{l}270 \\
264 \\
263 \\
262 \\
241\end{array}$ & $\begin{array}{l}150 \\
200 \\
200 \\
200 \\
200\end{array}$ & $\begin{array}{l}L \\
L \\
L \\
L \\
L\end{array}$ & $\begin{array}{l}-- \\
-- \\
-- \\
-- \\
--\end{array}$ & $\begin{array}{l}L \\
L \\
L \\
L\end{array}$ & $\begin{array}{l}\text { Grn, jọint, Fe stain. } \\
\text { Vol, green stain. }\end{array}$ \\
\hline $\begin{array}{l}216-5 \\
217-5 \\
218-5 \\
219-5 \\
220-5\end{array}$ & $\begin{array}{l}10 \\
10 \\
10 \\
10 \\
10\end{array}$ & $\begin{array}{r}5 \\
30 \\
30 \\
50 \\
2\end{array}$ & $\begin{array}{rl}{ }^{2} L & (25) \\
2 & 28 \\
2 & 27 \\
2 & 25 \\
{ }^{2} L(25)\end{array}$ & $\begin{array}{r}5 \\
7 \\
10 \\
10 \\
5\end{array}$ & $\begin{array}{r}1,000 \\
700 \\
1,000 \\
1,000 \\
700\end{array}$ & $\begin{array}{l}50 \\
50 \\
70 \\
50 \\
50\end{array}$ & $\begin{array}{l}5 L(20) \\
5 L(20) \\
5 L(20) \\
5 L(20) \\
5 L(20)\end{array}$ & $\begin{array}{r}7 \\
7 \\
10 \\
10 \\
7\end{array}$ & $\begin{array}{l}233 \\
249 \\
245 \\
256 \\
268\end{array}$ & $\begin{array}{l}50 \\
100 \\
100 \\
150 \\
100\end{array}$ & $\begin{array}{l}\mathrm{L} \\
\mathrm{L} \\
\mathrm{L} \\
\mathrm{L} \\
\mathrm{L}\end{array}$ & $\begin{array}{l}-- \\
-- \\
-- \\
--\end{array}$ & $\begin{array}{l}\mathrm{L} \\
\mathrm{L} \\
\mathrm{L} \\
\mathrm{L} \\
\mathrm{L}\end{array}$ & $\begin{array}{l}--- \\
\cdots- \\
--- \\
---\end{array}$ \\
\hline $\begin{array}{l}221-5 \\
222-5 \\
223-5 \\
224-5 \\
225-5\end{array}$ & $\begin{array}{c}10 \\
L \\
L \\
L \\
L\end{array}$ & $\begin{array}{r}50 \\
2 \\
50 \\
30 \\
2\end{array}$ & $\begin{array}{l}{ }^{2} L(25) \\
{ }^{2} L(25) \\
2 L(25) \\
2 L(25) \\
2 L(25) \\
2 L(25)\end{array}$ & $\begin{array}{r}10 \\
5 \\
20 \\
15 \\
5\end{array}$ & $\begin{array}{r}1,000 \\
700 \\
1,500 \\
1,000 \\
700\end{array}$ & $\begin{array}{r}70 \\
30 \\
100 \\
100 \\
50\end{array}$ & $\begin{array}{l}5_{L}(20) \\
5_{L}(20) \\
5_{L}(20) \\
5_{L}(20) \\
5_{L}(20)\end{array}$ & $\begin{array}{r}10 \\
5 \\
15 \\
15 \\
5\end{array}$ & $\begin{array}{c}230 \\
228 \\
252 \\
2 L(25) \\
233\end{array}$ & $\begin{array}{r}100 \\
30 \\
100 \\
150 \\
50\end{array}$ & $\begin{array}{l}\mathrm{L} \\
\mathrm{L} \\
\mathrm{L} \\
\mathrm{L} \\
\mathrm{L}\end{array}$ & $\begin{array}{l}-- \\
-- \\
-- \\
-- \\
--\end{array}$ & $\begin{array}{l}L \\
L \\
L \\
L \\
L\end{array}$ & $\begin{array}{l}-\cdots \\
--- \\
--- \\
---\end{array}$ \\
\hline
\end{tabular}


G34 STUDIES RELATED TO WILDERNESS-PRIMITIVE AREAS

TABLE 4.-Analyses of samples from the Emigrant

\begin{tabular}{|c|c|c|c|c|c|c|c|c|c|c|c|c|c|c|}
\hline \multirow{2}{*}{$\begin{array}{l}\text { Sample } \\
\text { and } \\
\text { type }\end{array}$} & \multicolumn{4}{|c|}{$\begin{array}{c}\text { Semiquantitative spectrographic } \\
\text { analyses (percent) }\end{array}$} & \multicolumn{10}{|c|}{$\begin{array}{c}\text { Semiquantitative spectrographic } \\
\text { analyses (ppm) }\end{array}$} \\
\hline & $\begin{array}{c}\text { Fe } \\
(0.05)\end{array}$ & $\begin{array}{c}\mathrm{Mg} \\
(0.005)\end{array}$ & $\begin{array}{c}\mathrm{Ca} \\
(0.05)\end{array}$ & $(0.001)$ & $\begin{array}{l}M n \\
(20)\end{array}$ & $\begin{array}{l}\mathrm{Ag} \\
(0.5)\end{array}$ & $\begin{array}{c}B \\
(10)\end{array}$ & $\begin{array}{l}B a \\
(20)\end{array}$ & $\begin{array}{l}\mathrm{Be} \\
(1)\end{array}$ & $\begin{array}{l}\text { Co } \\
(5)\end{array}$ & $\begin{array}{l}c r \\
(5)\end{array}$ & $\begin{array}{l}\mathrm{Cu} \\
(2)\end{array}$ & $\begin{array}{l}\text { La } \\
(20)\end{array}$ & $\begin{array}{l}\text { Mo } \\
(5)\end{array}$ \\
\hline $\begin{array}{l}226-5 \\
227-S \\
228-A \\
229-5 \\
230-5\end{array}$ & $\begin{array}{l}3 \\
3 \\
3 \\
3 \\
3\end{array}$ & $\begin{array}{l}1 \\
1 \\
.5 \\
1.7\end{array}$ & $\begin{array}{l}1.5 \\
2 \\
2 \\
1.5 \\
1.5\end{array}$ & $\begin{array}{l}0.5 \\
.5 \\
.5 \\
.5 \\
.5\end{array}$ & $\begin{array}{r}1,000 \\
1,000 \\
500 \\
300 \\
500\end{array}$ & $\begin{array}{r}20.4 \\
2.4 \\
2.4 \\
{ }^{2} L(.2) \\
2.3\end{array}$ & $\begin{array}{c}N \\
N \\
N \\
10 \\
N\end{array}$ & $\begin{array}{r}1,000 \\
1,000 \\
500 \\
1,500 \\
1,000\end{array}$ & $\begin{array}{l}\mathrm{L} \\
\mathrm{L} \\
\mathrm{I} \\
\mathrm{L} \\
\mathrm{L}\end{array}$ & $\begin{array}{l}30 \\
30 \\
20 \\
30 \\
30\end{array}$ & $\begin{array}{l}50 \\
50 \\
10 \\
50 \\
30\end{array}$ & $\begin{array}{r}222 \\
215 \\
{ }^{2} L(10) \\
218 \\
{ }^{2} 20\end{array}$ & $\begin{array}{l}30 \\
30 \\
30 \\
20 \\
30\end{array}$ & $\begin{array}{l}5 L(2) \\
5 L(2) \\
5 L(2) \\
5 L(2) \\
5 L(2)\end{array}$ \\
\hline $\begin{array}{l}231-5 \\
232-5 \\
233-5 \\
234-5 \\
235-5\end{array}$ & $\begin{array}{l}3 \\
3 \\
3 \\
2 \\
3\end{array}$ & $\begin{array}{l}.7 \\
.7 \\
.5 \\
.3 \\
.7\end{array}$ & $\begin{array}{l}1 \\
1 \\
1 \\
1 \\
1\end{array}$ & $\begin{array}{l}.3 \\
.5 \\
.5 \\
.3 \\
.5\end{array}$ & $\begin{array}{r}300 \\
1,000 \\
300 \\
300 \\
500\end{array}$ & $\begin{array}{c}{ }^{2} \mathrm{~L}(.2) \\
2.2 \\
{ }^{2} L(.2) \\
{ }^{2} L(.2) \\
2.3\end{array}$ & $\begin{array}{r}10 \\
10 \\
20 \\
10 \\
L\end{array}$ & $\begin{array}{l}1,500 \\
1,500 \\
1,500 \\
1,500 \\
2,000\end{array}$ & $\begin{array}{l}1 \\
1 \\
1 \\
1 \\
1.5\end{array}$ & $\begin{array}{l}30 \\
30 \\
30 \\
10 \\
50\end{array}$ & $\begin{array}{l}30 \\
30 \\
50 \\
15 \\
30\end{array}$ & $\begin{array}{l}210 \\
227 \\
225 \\
214 \\
294\end{array}$ & $\begin{array}{l}20 \\
20 \\
50 \\
30 \\
50\end{array}$ & $\begin{array}{l}5 L(2) \\
5 L(2) \\
5 L(2) \\
5 L(2) \\
5 L(2)\end{array}$ \\
\hline $\begin{array}{l}236-5 \\
237-5 \\
238-5 \\
239-5 \\
240-5\end{array}$ & $\begin{array}{l}2 \\
3 \\
2 \\
3 \\
2\end{array}$ & $\begin{array}{l}.7 \\
.7 \\
.5 \\
.7 \\
.5\end{array}$ & $\begin{array}{r}1.5 \\
.7 \\
.5 \\
.7 \\
.5\end{array}$ & $\begin{array}{l}.2 \\
.5 \\
.3 \\
.5 \\
.3\end{array}$ & $\begin{array}{l}300 \\
500 \\
500 \\
500 \\
300\end{array}$ & $\begin{array}{r}2.2 \\
2.3 \\
{ }^{2} L(.2) \\
2.4 \\
2.2\end{array}$ & $\begin{array}{l}20 \\
10 \\
10 \\
10 \\
L\end{array}$ & $\begin{array}{l}1,500 \\
1,500 \\
1,500 \\
1,500 \\
1,500\end{array}$ & $\begin{array}{l}1 \\
1.5 \\
1.5 \\
1 \\
1\end{array}$ & $\begin{array}{l}20 \\
50 \\
20 \\
50 \\
20\end{array}$ & $\begin{array}{l}20 \\
30 \\
15 \\
20 \\
15\end{array}$ & $\begin{array}{c}{ }^{2} L(10) \\
235 \\
{ }^{2} L(10) \\
217 \\
{ }^{2} L(10) \\
{ }^{2}(10)\end{array}$ & $\begin{array}{r}30 \\
50 \\
30 \\
30 \\
L\end{array}$ & $\begin{array}{l}5 L(2) \\
5 L(2) \\
5 L(2) \\
5 L(2) \\
5 L(2)\end{array}$ \\
\hline $\begin{array}{l}241-5 \\
242-5 \\
243-5 \\
244-5 \\
245-5\end{array}$ & $\begin{array}{l}2 \\
2 \\
1.5 \\
1.5 \\
5\end{array}$ & $\begin{array}{r}.5 \\
1.5 \\
.7 \\
.7\end{array}$ & $\begin{array}{l}1 \\
3 \\
3 \\
3 \\
5\end{array}$ & $\begin{array}{l}.3 \\
.3 \\
.2 \\
.3 \\
.7\end{array}$ & $\begin{array}{l}300 \\
300 \\
200 \\
200 \\
500\end{array}$ & $\begin{array}{l}{ }^{2}{ }^{3} \\
N(1)^{3} \\
N(1) \\
N(1) \\
N(1)\end{array}$ & $\begin{array}{c}\text { N } \\
-- \\
--\end{array}$ & $\begin{array}{r}1,500 \\
1,500 \\
1,500 \\
1,500 \\
700\end{array}$ & $\begin{array}{l}\text { I } \\
N \\
N \\
N \\
N\end{array}$ & $\begin{array}{r}20 \\
5 \\
5 \\
3 \\
10\end{array}$ & $\begin{array}{r}20 \\
1 \\
1 \\
1 \\
2\end{array}$ & $\begin{array}{r}215 \\
5 \\
5 \\
3 \\
7\end{array}$ & $\begin{array}{c}20 \\
N(30) \\
N(30) \\
N(30) \\
N(30)\end{array}$ & $\begin{array}{l}5 L(2) \\
N(3) \\
N(3) \\
N(3) \\
N(3)\end{array}$ \\
\hline $\begin{array}{l}246-V \\
247-S \\
248-A \\
249-S \\
250-A\end{array}$ & $\begin{array}{l}2 \\
1.5 \\
7 \\
5 \\
7\end{array}$ & $\begin{array}{l}5 \\
1 \\
7 \\
1.5 \\
1.5\end{array}$ & $\begin{array}{l}7 \\
6 \\
6 \\
6\end{array}$ & $\begin{array}{l}.01 \\
.2 \\
.7 \\
.7 \\
.7\end{array}$ & $\begin{array}{r}3,000 \\
300 \\
2,000 \\
700 \\
1,500\end{array}$ & $\begin{array}{l}N(1) \\
N(1) \\
N(1) \\
N(1) \\
N(1)\end{array}$ & $\begin{array}{l}\cdots \\
\cdots \\
\cdots\end{array}$ & $\begin{array}{r}70 \\
700 \\
700 \\
700 \\
300\end{array}$ & $\begin{array}{l}N \\
N \\
1 \\
N \\
N\end{array}$ & $\begin{array}{r}5 \\
5 \\
15 \\
7 \\
N(3)\end{array}$ & $\begin{array}{l}3 \\
15 \\
70 \\
30 \\
70\end{array}$ & $\begin{array}{l}1 \\
10 \\
30 \\
30 \\
10\end{array}$ & $\begin{array}{c}N(30) \\
N(30) \\
150 \\
N(30) \\
70\end{array}$ & $\begin{array}{l}N(3) \\
N(3) \\
N(3) \\
N(3) \\
N(3)\end{array}$ \\
\hline $\begin{array}{l}251-c \\
252-5 \\
253-5 \\
254-5 \\
255-5\end{array}$ & $\begin{array}{l}7 \\
3 \\
1.5 \\
1 \\
1.5\end{array}$ & $\begin{array}{l}3 \\
3 \\
.3 \\
.3 \\
.7\end{array}$ & $\begin{array}{l}G \\
G \\
1 \\
1 \\
3\end{array}$ & $\begin{array}{l}.03 \\
.5 \\
.1 \\
.2 \\
.3\end{array}$ & $\begin{array}{r}15,000 \\
1,500 \\
200 \\
200 \\
500\end{array}$ & $\begin{array}{l}20 \\
N(1) \\
N(1) \\
N(1) \\
N(1)\end{array}$ & $\begin{array}{l}\cdots \\
\cdots \\
\cdots\end{array}$ & $\begin{array}{r}30 \\
700 \\
700 \\
1,500 \\
1,500\end{array}$ & $\begin{array}{l}N \\
N \\
N \\
N \\
N\end{array}$ & $\begin{array}{c}20 \\
5 \\
N(3) \\
N(3) \\
3\end{array}$ & $\begin{array}{r}5 \\
30 \\
2 \\
3 \\
2\end{array}$ & $\begin{array}{r}100 \\
10 \\
5 \\
1 \\
3\end{array}$ & $\begin{array}{c}N(30) \\
30 \\
N(30) \\
30 \\
N(30)\end{array}$ & $\begin{array}{l}15 \\
N(3) \\
N(3) \\
N(3) \\
L(3)\end{array}$ \\
\hline $\begin{array}{l}256-C \\
257-S \\
258-S \\
259-S \\
260-S\end{array}$ & $\begin{array}{l}G \\
1.5 \\
5 \\
5 \\
1.5\end{array}$ & $\begin{array}{l}1 \\
1 \\
3 \\
2 \\
.3\end{array}$ & $\begin{array}{l}3 \\
3 \\
7 \\
5 \\
5\end{array}$ & $\begin{array}{c}10 \\
.3 \\
.7 \\
.7 \\
.15\end{array}$ & $\begin{array}{l}700 \\
300 \\
300 \\
150 \\
200\end{array}$ & $\begin{array}{l}N(1) \\
N(1) \\
N(1) \\
N(1) \\
N(1)\end{array}$ & $\begin{array}{l}\cdots \\
\cdots \\
\cdots \\
\cdots\end{array}$ & $\begin{array}{r}70 \\
1,500 \\
1,500 \\
1,500 \\
1,500\end{array}$ & $\begin{array}{l}\mathrm{N} \\
\mathrm{N} \\
\mathrm{N} \\
\mathrm{N} \\
\mathrm{N}\end{array}$ & $\begin{array}{r}20 \\
5 \\
10 \\
10 \\
3\end{array}$ & $\begin{array}{r}30 \\
2 \\
10 \\
5 \\
1\end{array}$ & $\begin{array}{r}100 \\
5 \\
15 \\
7 \\
2\end{array}$ & $\begin{array}{r}150 \\
50 \\
30 \\
50 \\
N(30)\end{array}$ & $\begin{array}{l}N(3) \\
N(3) \\
N(3) \\
N(3) \\
N(3)\end{array}$ \\
\hline $\begin{array}{l}261-S \\
262-s \\
263-c \\
264-C \\
265-s\end{array}$ & $\begin{array}{l}1.5 \\
1.5 \\
G \\
G \\
2\end{array}$ & $\begin{array}{l}1 \\
3 \\
5 \\
2\end{array}$ & $\begin{array}{l}5 \\
5 \\
7 \\
7 \\
7\end{array}$ & $\begin{array}{c}.3 \\
10^{.2} \\
.3\end{array}$ & $\begin{array}{r}700 \\
150 \\
1,500 \\
1,000 \\
500\end{array}$ & $\begin{array}{l}N(1) \\
N(1) \\
N(1) \\
N(1) \\
N(1)\end{array}$ & $\begin{array}{l}\cdots \\
\cdots \\
\cdots \\
--\end{array}$ & $\begin{array}{r}1,500 \\
1,500 \\
700 \\
200 \\
1,500\end{array}$ & $\begin{array}{l}\mathrm{N} \\
\mathrm{N} \\
\mathrm{N} \\
\mathrm{N} \\
\mathrm{N}\end{array}$ & $\begin{array}{r}3 \\
3 \\
15 \\
30 \\
10\end{array}$ & $\begin{array}{r}1 \\
1 \\
70 \\
100 \\
10\end{array}$ & $\begin{array}{r}5 \\
3 \\
50 \\
50 \\
5\end{array}$ & $\begin{array}{c}N(30) \\
N(30) \\
300 \\
500 \\
N(30)\end{array}$ & $\begin{array}{l}N(3) \\
N(3) \\
N(3) \\
N(3) \\
N(3)\end{array}$ \\
\hline $\begin{array}{l}266-5 \\
267-5 \\
268-5 \\
269-5 \\
270-5\end{array}$ & $\begin{array}{l}1.5 \\
1.5 \\
2 \\
2 \\
1.5\end{array}$ & $\begin{array}{l}2 \\
1 \\
1 \\
2 \\
.5\end{array}$ & $\begin{array}{l}5 \\
5 \\
3 \\
5 \\
5\end{array}$ & $\begin{array}{l}.2 \\
.15 \\
.2 \\
.2 \\
.3\end{array}$ & $\begin{array}{l}300 \\
300 \\
500 \\
500 \\
200\end{array}$ & $\begin{array}{l}N(1) \\
N(1) \\
N(1) \\
N(1) \\
N(1)\end{array}$ & $\begin{array}{l}-- \\
-- \\
-- \\
-- \\
--\end{array}$ & $\begin{array}{l}1,500 \\
1,500 \\
1,500 \\
1,000 \\
1,500\end{array}$ & $\begin{array}{l}\mathrm{N} \\
\mathrm{N} \\
\mathrm{N} \\
\mathrm{N} \\
\mathrm{N}\end{array}$ & $\begin{array}{l}5 \\
3 \\
3 \\
5 \\
3\end{array}$ & $\begin{array}{l}2 \\
3 \\
N \\
2 \\
1\end{array}$ & $\begin{array}{l}3 \\
3 \\
3 \\
5 \\
3\end{array}$ & $\begin{array}{l}N(30) \\
N(30) \\
N(30) \\
N(30) \\
N(30)\end{array}$ & $\begin{array}{l}N(3) \\
N(3) \\
N(3) \\
N(3) \\
N(3)\end{array}$ \\
\hline $\begin{array}{l}271-5 \\
272-5 \\
273-5 \\
274-50 \\
275-5\end{array}$ & $\begin{array}{l}2 \\
2 \\
1 \\
3 \\
2\end{array}$ & $\begin{array}{l}1 \\
.7 \\
.3 \\
.3 \\
.3\end{array}$ & $\begin{array}{l}5 \\
5 \\
3 \\
5 \\
5\end{array}$ & $\begin{array}{l}.3 \\
.3 \\
.3 \\
.3 \\
.5\end{array}$ & $\begin{array}{l}500 \\
500 \\
200 \\
300 \\
200\end{array}$ & $\begin{array}{l}N(1) \\
N(1) \\
N(1) \\
N(1) \\
N(1)\end{array}$ & $\begin{array}{l}-- \\
-- \\
-- \\
--\end{array}$ & $\begin{array}{l}1,500 \\
1,500 \\
1,500 \\
1,500 \\
1,500\end{array}$ & $\begin{array}{l}\mathrm{N} \\
\mathrm{N} \\
\mathrm{N} \\
\mathrm{N} \\
\mathrm{N}\end{array}$ & $\begin{array}{c}5 \\
3 \\
N(3) \\
3 \\
2\end{array}$ & $\begin{array}{c}1 \\
L(1) \\
N \\
2 \\
L(1)\end{array}$ & $\begin{array}{l}2 \\
1 \\
2 \\
7 \\
2\end{array}$ & $\begin{array}{r}30 \\
30 \\
N(30) \\
100 \\
N(30)\end{array}$ & $\begin{array}{l}N(3) \\
N(3) \\
N(3) \\
N(3) \\
N(3)\end{array}$ \\
\hline $\begin{array}{l}276-S \\
277-V \\
278-A \\
278 a-V \\
279-R\end{array}$ & $\begin{array}{l}3 \\
7 \\
3 \\
7\end{array}$ & $\begin{array}{l}.7 \\
.15 \\
.7 \\
3 \\
2\end{array}$ & $\begin{array}{l}5 \\
5^{.03} \\
3 \\
5\end{array}$ & $\begin{array}{l}.5 \\
.005 \\
.5 \\
.02 \\
.1\end{array}$ & $\begin{array}{r}200 \\
700 \\
G \\
G \\
G\end{array}$ & $\begin{array}{c}N(1) \\
5 \\
2.5 \\
2.3 \\
21.7\end{array}$ & $\begin{array}{l}-- \\
\cdots \\
N \\
L\end{array}$ & $\begin{array}{r}1,500 \\
150 \\
150 \\
N \\
\mathrm{~L}\end{array}$ & $\begin{array}{l}N \\
N \\
L \\
2 \\
1\end{array}$ & $\begin{array}{l}3 \\
50 \\
10 \\
15 \\
15\end{array}$ & $\begin{array}{r}2 \\
70 \\
100 \\
L \\
20\end{array}$ & $\begin{array}{r}5 \\
70 \\
15 \\
2 \\
200\end{array}$ & $\begin{array}{c}30 \\
N(30) \\
50 \\
N \\
N\end{array}$ & $\begin{array}{l}N(3) \\
N(3) \\
100 \\
200 \\
100\end{array}$ \\
\hline $\begin{array}{l}279-A \\
280-S \\
281-S \\
282-S \\
283-S\end{array}$ & $\begin{array}{l}2 \\
3 \\
2 \\
5 \\
2\end{array}$ & $\begin{array}{l}1.5 \\
1.5 \\
1.5 \\
3 \\
1\end{array}$ & $\begin{array}{l}3 \\
1 \\
1 \\
1 \\
.7\end{array}$ & $\begin{array}{l}.2 \\
.3 \\
.3 \\
.5 \\
.2\end{array}$ & $\begin{array}{r}700 \\
1,000 \\
500 \\
700 \\
300\end{array}$ & 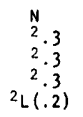 & $\begin{array}{l}15 \\
10 \\
10 \\
10 \\
10\end{array}$ & $\begin{array}{l}500 \\
500 \\
700 \\
700 \\
500\end{array}$ & $\begin{array}{l}1.5 \\
L \\
L \\
L \\
L\end{array}$ & $\begin{array}{r}7 \\
15 \\
10 \\
20 \\
5\end{array}$ & $\begin{array}{r}50 \\
10 \\
15 \\
5 \\
1\end{array}$ & $\begin{array}{l}30 \\
15 \\
10 \\
20 \\
10\end{array}$ & $\begin{array}{r}50 \\
20 \\
L \\
L \\
L\end{array}$ & $\begin{array}{c}N \\
15 \\
L \\
N \\
N\end{array}$ \\
\hline
\end{tabular}




\begin{tabular}{|c|c|c|c|c|c|c|c|c|c|c|}
\hline \multirow{2}{*}{$\begin{array}{l}\text { Sample } \\
\text { and } \\
\text { type }\end{array}$} & \multicolumn{7}{|c|}{$\begin{array}{l}\text { Semiquantitative spectrographic } \\
\text { analyses (ppm)--Continued }\end{array}$} & \multicolumn{2}{|r|}{$\begin{array}{c}\text { Chemical analyses } \\
(\mathrm{ppm})\end{array}$} & \multirow{2}{*}{ Remarks } \\
\hline & $\begin{array}{ll}\mathrm{Nb} & \mathrm{Ni} \\
(10) & (2)\end{array}$ & $\begin{array}{cc}\mathrm{Pb} & \mathrm{Sc} \\
(10) & (5)\end{array}$ & $\begin{array}{l}S r \\
(50)\end{array}$ & $\begin{array}{l}v \\
(10)\end{array}$ & $\underset{(50)}{w}$ & $\begin{array}{c}y \\
(5)\end{array}$ & $\begin{array}{l}\operatorname{Zn} \\
(200)\end{array}$ & $\begin{array}{l}2 r \\
(10)\end{array}$ & 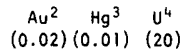 & \\
\hline
\end{tabular}

\begin{tabular}{|c|c|c|c|c|c|c|c|c|c|c|c|c|c|c|}
\hline $\begin{array}{l}226-S \\
227-S \\
228-A \\
229-S \\
230-S\end{array}$ & $\begin{array}{l}L \\
L \\
L \\
L \\
L\end{array}$ & $\begin{array}{l}30 \\
50 \\
30 \\
50 \\
30\end{array}$ & $\begin{array}{c}{ }^{2} L(25) \\
{ }^{2} L(25) \\
{ }^{2} L(25) \\
230 \\
228\end{array}$ & $\begin{array}{l}20 \\
20 \\
20 \\
20 \\
20\end{array}$ & $\begin{array}{r}1,000 \\
1,000 \\
700 \\
700 \\
1,000\end{array}$ & $\begin{array}{r}70 \\
70 \\
70 \\
100 \\
100\end{array}$ & $\begin{array}{l}{ }^{5} L(20) \\
5 L(20) \\
5 L(20) \\
5 L(20) \\
5 L(20)\end{array}$ & $\begin{array}{l}15 \\
10 \\
15 \\
10 \\
10\end{array}$ & $\begin{array}{l}255 \\
239 \\
247 \\
250 \\
247\end{array}$ & $\begin{array}{l}100 \\
100 \\
200 \\
150 \\
150\end{array}$ & $\begin{array}{l}\mathrm{L} \\
\mathrm{L} \\
\mathrm{L} \\
\mathrm{L} \\
\mathrm{L}\end{array}$ & $\begin{array}{l}-- \\
-- \\
-- \\
-- \\
--\end{array}$ & $\begin{array}{l}\mathrm{L} \\
\mathrm{L} \\
\mathrm{L} \\
\mathrm{L} \\
\mathrm{L}\end{array}$ & Vol, tuff, green. \\
\hline $\begin{array}{l}231-5 \\
232-5 \\
233-5 \\
234-5 \\
235-5\end{array}$ & $\begin{array}{l}L \\
L \\
10 \\
10 \\
10\end{array}$ & $\begin{array}{l}30 \\
30 \\
30 \\
10 \\
50\end{array}$ & $\begin{array}{l}225 \\
249 \\
244 \\
240 \\
296\end{array}$ & $\begin{array}{l}15 \\
15 \\
15 \\
10 \\
20\end{array}$ & $\begin{array}{r}1,000 \\
1,000 \\
1,000 \\
500 \\
700\end{array}$ & $\begin{array}{r}100 \\
100 \\
100 \\
70 \\
150\end{array}$ & $\begin{array}{l}5_{L}(20) \\
5_{L}(20) \\
5_{L}(20) \\
5_{L}(20) \\
5_{L}(20)\end{array}$ & $\begin{array}{r}10 \\
10 \\
15 \\
7 \\
15\end{array}$ & $\begin{array}{r}251 \\
2110 \\
290 \\
270 \\
2250\end{array}$ & $\begin{array}{l}100 \\
150 \\
200 \\
100 \\
150\end{array}$ & $\begin{array}{l}L \\
L \\
L \\
L\end{array}$ & $\begin{array}{l}-- \\
-- \\
-- \\
-- \\
--\end{array}$ & $\begin{array}{l}L \\
L \\
L \\
L \\
L\end{array}$ & $\begin{array}{l}--- \\
--- \\
--- \\
---\end{array}$ \\
\hline $\begin{array}{l}236-5 \\
237-5 \\
238-5 \\
239-5 \\
240-5\end{array}$ & $\begin{array}{r}\mathrm{L} \\
10 \\
10 \\
10 \\
\mathrm{~L}\end{array}$ & $\begin{array}{l}20 \\
50 \\
10 \\
50 \\
10\end{array}$ & $\begin{array}{c}{ }^{2} L(25) \\
239 \\
240 \\
228 \\
{ }^{2} L(25)\end{array}$ & $\begin{array}{l}15 \\
20 \\
15 \\
15 \\
10\end{array}$ & $\begin{array}{r}1,500 \\
700 \\
500 \\
1,000 \\
700\end{array}$ & $\begin{array}{r}70 \\
100 \\
70 \\
100 \\
70\end{array}$ & $\begin{array}{l}{ }^{5} L(20) \\
5 L(20) \\
5 L(20) \\
5 L(20) \\
5 L(20) \\
5 L(20)\end{array}$ & $\begin{array}{r}10 \\
15 \\
10 \\
15 \\
7\end{array}$ & $\begin{array}{c}{ }^{2} L(25) \\
272 \\
250 \\
244 \\
{ }^{2} L(25)\end{array}$ & $\begin{array}{l}100 \\
150 \\
100 \\
150 \\
100\end{array}$ & $\begin{array}{l}L \\
L \\
L \\
L \\
L\end{array}$ & $\begin{array}{l}-- \\
-- \\
-- \\
--\end{array}$ & $\begin{array}{l}L \\
L \\
L \\
L\end{array}$ & $\begin{array}{l}--- \\
--- \\
--- \\
-\cdots\end{array}$ \\
\hline $\begin{array}{l}241-5 \\
242-5 \\
243-5 \\
244-5 \\
245-5\end{array}$ & $\begin{array}{r}10 \\
N \\
N \\
N \\
N\end{array}$ & $\begin{array}{c}20 \\
N(3) \\
N(3) \\
N(3) \\
3\end{array}$ & $\begin{array}{r}229 \\
20 \\
20 \\
20 \\
20\end{array}$ & $\begin{array}{l}15 \\
-- \\
-- \\
-- \\
--\end{array}$ & $\begin{array}{r}1,000 \\
700 \\
700 \\
700 \\
700\end{array}$ & $\begin{array}{l}50 \\
30 \\
20 \\
30 \\
70\end{array}$ & $\begin{array}{l}5 L(20) \\
N(100) \\
N(100) \\
N(100) \\
N(100)\end{array}$ & $\begin{array}{c}7 \\
N(10) \\
N(10) \\
10 \\
10\end{array}$ & $\begin{array}{l}250 \\
254 \\
249 \\
246 \\
280\end{array}$ & $\begin{array}{r}150 \\
70 \\
50 \\
100 \\
100\end{array}$ & $\begin{array}{l}L \\
L \\
L \\
L \\
L\end{array}$ & $\begin{array}{l}-- \\
-- \\
-- \\
--\end{array}$ & $\begin{array}{l}L \\
-- \\
-- \\
-- \\
--\end{array}$ & $\begin{array}{c}(T a, T e, P d, P t), N . \\
\text { Do. } \\
\text { Do. } \\
\text { Do. }\end{array}$ \\
\hline $\begin{array}{l}246-V \\
247-S \\
248-A \\
249-S \\
250-A\end{array}$ & $\begin{array}{r}N \\
10 \\
20 \\
N \\
10\end{array}$ & $\begin{array}{r}10 \\
10 \\
30 \\
10 \\
5\end{array}$ & $\begin{array}{r}N \\
10 \\
10 \\
N \\
10\end{array}$ & $\begin{array}{l}-- \\
-- \\
-- \\
--\end{array}$ & $\begin{array}{r}20 \\
70 \\
300 \\
70 \\
500\end{array}$ & $\begin{array}{r}30 \\
20 \\
100 \\
100 \\
100\end{array}$ & $\begin{array}{l}N(100) \\
N(100) \\
N(100) \\
N(100) \\
N(100)\end{array}$ & $\begin{array}{c}N(10) \\
10 \\
70 \\
10 \\
50\end{array}$ & $\begin{array}{l}-= \\
235 \\
268 \\
252 \\
272\end{array}$ & $\begin{array}{r}N \\
150 \\
300 \\
150 \\
500\end{array}$ & $\begin{array}{l}L \\
L \\
L \\
L\end{array}$ & $\begin{array}{l}-- \\
-- \\
-- \\
-- \\
--\end{array}$ & $\begin{array}{l}-- \\
-- \\
-- \\
--\end{array}$ & $\begin{array}{l}\text { Met, shear zone, and }{ }^{\mathrm{Do}} \\
(\mathrm{Ta}, \mathrm{Te}, \mathrm{Pd}, \mathrm{Pt}), \mathrm{N} . \\
\text { Met, tactite, and Do. } \\
\left({ }^{8}\right) .\end{array}$ \\
\hline $\begin{array}{l}251-c \\
252-5 \\
253-5 \\
254-5 \\
255-5\end{array}$ & $\begin{array}{r}50 \\
N \\
N \\
N \\
10\end{array}$ & $\begin{array}{r}3 \\
15 \\
N(3) \\
1 \\
N(3)\end{array}$ & $\begin{array}{r}200 \\
N \\
30 \\
10 \\
20\end{array}$ & $\begin{array}{l}-- \\
-- \\
-- \\
--\end{array}$ & $\begin{array}{r}20 \\
150 \\
150 \\
150 \\
300\end{array}$ & $\begin{array}{l}30 \\
50 \\
15 \\
10 \\
50\end{array}$ & $\begin{array}{l}7,000 \\
N(100) \\
N(100) \\
N(100) \\
N(100)\end{array}$ & $\begin{array}{c}15 \\
30 \\
N(10) \\
L(10) \\
15\end{array}$ & $\begin{array}{r}217,000 \\
2140 \\
240 \\
230 \\
238\end{array}$ & $\begin{array}{r}30 \\
500 \\
50 \\
150 \\
70\end{array}$ & $\begin{array}{l}L \\
L \\
L \\
L \\
L\end{array}$ & $\begin{array}{l}-- \\
-- \\
-- \\
-- \\
--\end{array}$ & $\begin{array}{l}-- \\
-- \\
-- \\
--\end{array}$ & $\begin{array}{l}-- \\
\cdots \\
\cdots-\end{array}$ \\
\hline $\begin{array}{l}256-\mathrm{C} \\
257-5 \\
258-5 \\
259-5 \\
260-5\end{array}$ & $\begin{array}{r}20 \\
N \\
N \\
N \\
N\end{array}$ & $\begin{array}{c}15 \\
N(3) \\
10 \\
5 \\
N(3)\end{array}$ & $\begin{array}{l}10 \\
20 \\
20 \\
10 \\
10\end{array}$ & $\begin{array}{l}-- \\
-- \\
-- \\
--\end{array}$ & $\begin{array}{r}30 \\
700 \\
700 \\
700 \\
500\end{array}$ & $\begin{array}{r}500 \\
50 \\
100 \\
70 \\
30\end{array}$ & $\begin{array}{l}N(100) \\
N(100) \\
N(100) \\
N(100) \\
N(100)\end{array}$ & $\begin{array}{r}70 \\
10 \\
10 \\
15 \\
N(10)\end{array}$ & $\begin{array}{r}2152 \\
245 \\
272 \\
280 \\
238\end{array}$ & $\begin{array}{r}700 \\
70 \\
100 \\
100 \\
10\end{array}$ & $\begin{array}{l}L \\
L \\
L \\
L \\
L\end{array}$ & $\begin{array}{l}-- \\
-- \\
-- \\
--\end{array}$ & $\begin{array}{l}-- \\
-- \\
-- \\
--\end{array}$ & $\begin{array}{l}--- \\
--- \\
---\end{array}$ \\
\hline $\begin{array}{l}261-S \\
262-S \\
263-c \\
264-c \\
265-S\end{array}$ & $\begin{array}{r}N \\
N \\
30 \\
50 \\
N\end{array}$ & $\begin{array}{c}N(3) \\
1 \\
5 \\
15 \\
3\end{array}$ & $\begin{array}{r}10 \\
20 \\
L \\
10 \\
10\end{array}$ & $\begin{array}{l}-- \\
-- \\
-- \\
-\end{array}$ & $\begin{array}{l}500 \\
700 \\
700 \\
300 \\
500\end{array}$ & $\begin{array}{r}50 \\
50 \\
500 \\
700 \\
70\end{array}$ & $\begin{array}{l}N(100) \\
N(100) \\
N(100) \\
N(100) \\
N(100)\end{array}$ & $\begin{array}{c}N(10) \\
N(10) \\
70 \\
70 \\
10\end{array}$ & $\begin{array}{r}252 \\
240 \\
2103 \\
2140 \\
275\end{array}$ & $\begin{array}{r}100 \\
50 \\
300 \\
1,500 \\
30\end{array}$ & $\begin{array}{l}L \\
L \\
L \\
L \\
L\end{array}$ & $\begin{array}{l}-- \\
-- \\
-- \\
-- \\
--\end{array}$ & $\begin{array}{l}-- \\
-- \\
-- \\
--\end{array}$ & $\begin{array}{c}\text { Met, st ream sand, Cherry } \\
\text { Do. } \\
-\end{array}$ \\
\hline $\begin{array}{l}266-5 \\
267-5 \\
268-5 \\
269-5 \\
270-5\end{array}$ & $\begin{array}{l}\mathrm{N} \\
\mathrm{N} \\
\mathrm{N} \\
\mathrm{N} \\
\mathrm{N}\end{array}$ & $\begin{array}{c}N(3) \\
1 \\
N(3) \\
2 \\
N(3)\end{array}$ & $\begin{array}{l}10 \\
20 \\
20 \\
20 \\
20\end{array}$ & $\begin{array}{l}-- \\
-- \\
-- \\
--\end{array}$ & $\begin{array}{l}700 \\
700 \\
500 \\
700 \\
700\end{array}$ & $\begin{array}{l}50 \\
30 \\
30 \\
70 \\
30\end{array}$ & $\begin{array}{l}N(100) \\
N(100) \\
N(100) \\
N(100) \\
N(100)\end{array}$ & $\begin{array}{l}10 \\
L(10) \\
N(10) \\
L(10) \\
15\end{array}$ & $\begin{array}{l}265 \\
245 \\
263 \\
268 \\
248\end{array}$ & $\begin{array}{r}100 \\
70 \\
150 \\
70 \\
50\end{array}$ & $\begin{array}{l}L \\
L \\
L \\
L \\
L\end{array}$ & $\begin{array}{l}-- \\
-- \\
-- \\
--\end{array}$ & $\begin{array}{l}-- \\
-- \\
-- \\
--\end{array}$ & $\begin{array}{l}--- \\
--- \\
--- \\
---\end{array}$ \\
\hline $\begin{array}{l}271-5 \\
272-5 \\
273-5 \\
274-50 \\
275-5\end{array}$ & $\begin{array}{r}N \\
N \\
N \\
10 \\
N\end{array}$ & $\begin{array}{l}N(3) \\
N(3) \\
N(3) \\
N(3) \\
N(3)\end{array}$ & $\begin{array}{l}15 \\
10 \\
10 \\
10 \\
10\end{array}$ & $\begin{array}{l}-- \\
-- \\
-- \\
--\end{array}$ & $\begin{array}{l}700 \\
700 \\
500 \\
700 \\
700\end{array}$ & $\begin{array}{l}30 \\
20 \\
20 \\
50 \\
30\end{array}$ & $\begin{array}{l}N(100) \\
N(100) \\
N(100) \\
N(100) \\
N(100)\end{array}$ & $\begin{array}{c}15 \\
L(10) \\
N(10) \\
10 \\
10\end{array}$ & $\begin{array}{l}270 \\
268 \\
235 \\
246 \\
242\end{array}$ & $\begin{array}{r}200 \\
150 \\
150 \\
200 \\
70\end{array}$ & $\begin{array}{l}L \\
L \\
L \\
L \\
L\end{array}$ & $\begin{array}{l}-- \\
-- \\
-- \\
--\end{array}$ & $\begin{array}{l}-- \\
-- \\
-- \\
--\end{array}$ & $\begin{array}{c}(\mathrm{Ta}, \mathrm{Te}, \mathrm{Pd}, \mathrm{Pt}), \mathrm{N} . \\
\text { Do. } \\
\text { Do. } \\
\text { Do. }\end{array}$ \\
\hline $\begin{array}{l}276-S \\
277-V \\
278-A \\
278 a-V \\
279-R\end{array}$ & $\begin{array}{c}N \\
N \\
10 \\
70 \\
L\end{array}$ & $\begin{array}{l}N(3) \\
200 \\
10 \\
7 \\
7\end{array}$ & $\begin{array}{r}10 \\
10 \\
10 \\
N \\
L\end{array}$ & $\begin{array}{c}-- \\
-- \\
\text { L }\end{array}$ & $\begin{array}{l}700 \\
N(5) \\
N \\
N \\
N\end{array}$ & $\begin{array}{r}70 \\
20 \\
70 \\
70 \\
300\end{array}$ & $\begin{array}{c}N(100) \\
N(100) \\
L \\
G \\
500\end{array}$ & $\begin{array}{c}10 \\
N(10) \\
70 \\
N \\
30\end{array}$ & $\begin{array}{r}253 \\
212 \\
2100 \\
2 L(25) \\
288\end{array}$ & $\begin{array}{r}50 \\
N \\
700 \\
N \\
100\end{array}$ & $\begin{array}{l}L \\
L \\
L \\
L\end{array}$ & $\begin{array}{l}-- \\
-- \\
0.09 \\
.07 \\
.09\end{array}$ & $\begin{array}{l}\cdots \\
-- \\
L \\
L\end{array}$ & 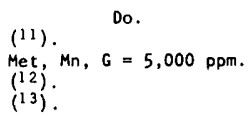 \\
\hline $\begin{array}{l}279-A \\
280-S \\
281-S \\
282-S \\
283-S\end{array}$ & $\begin{array}{l}10 \\
10 \\
10 \\
10 \\
10\end{array}$ & $\begin{array}{r}20 \\
20 \\
10 \\
15 \\
5\end{array}$ & $\begin{array}{l}N \\
20 \\
20 \\
20 \\
30\end{array}$ & $\begin{array}{r}10 \\
7 \\
7 \\
20 \\
1\end{array}$ & $\begin{array}{l}150 \\
200 \\
300 \\
500 \\
300\end{array}$ & $\begin{array}{r}50 \\
70 \\
70 \\
100 \\
50\end{array}$ & $\begin{array}{r}\mathrm{N} \\
70 \\
\mathrm{~N} \\
\mathrm{~N} \\
\mathrm{~N}\end{array}$ & $\begin{array}{l}20 \\
20 \\
15 \\
15 \\
10\end{array}$ & $\begin{array}{c}2 \mathrm{~N}(25) \\
257 \\
254 \\
258 \\
229\end{array}$ & $\begin{array}{l}150 \\
150 \\
100 \\
200 \\
100\end{array}$ & $\begin{array}{l}N \\
L \\
L \\
L \\
L\end{array}$ & $\begin{array}{l}\mathrm{N} \\
.01 \\
.04 \\
.04 \\
.03\end{array}$ & $\begin{array}{l}N \\
L \\
L \\
L \\
L\end{array}$ & $\begin{array}{l}\cdots \\
--- \\
\cdots \\
--\end{array}$ \\
\hline
\end{tabular}


TABLE 4.-Analyses of samples from the Emigrant

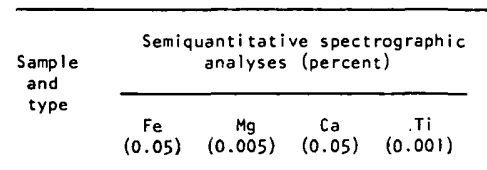

Semiquantitative spectrographic
analyses (ppm) 1

\begin{tabular}{cccccccccc}
\hline $\begin{array}{c}M n \\
(20)\end{array}$ & $\begin{array}{c}A g \\
(0.5)\end{array}$ & $(10)$ & $(20)$ & $(1)$ & $(5)$ & $(5)$ & $(2)$ & $(20)$ & $(5)$
\end{tabular}

\begin{tabular}{|c|c|c|c|c|c|c|c|c|c|c|c|c|c|c|}
\hline $\begin{array}{l}284-S \\
285-S \\
286-S \\
287-S \\
287 a-A\end{array}$ & $\begin{array}{l}3 \\
7 \\
3 \\
1 \\
1.5\end{array}$ & $\begin{array}{l}1.5 \\
5 \\
5 \\
1.5 \\
2\end{array}$ & $\begin{array}{l}0.7 \\
2 \\
2 \\
1 \\
.7\end{array}$ & $\begin{array}{r}2.3 \\
.5 \\
.5 \\
.2 \\
.2\end{array}$ & $\begin{array}{r}520 \\
1.220 \\
1.020 \\
300 \\
300\end{array}$ & $\begin{array}{r}0.2 \\
.6 \\
.7 \\
.2 \\
1.2\end{array}$ & $\begin{array}{l}L \\
12 \\
12 \\
10 \\
15\end{array}$ & $\begin{array}{r}302 \\
1.320 \\
728 \\
502 \\
1.005\end{array}$ & $\begin{array}{l}i \\
i \\
i \\
i\end{array}$ & $\begin{array}{l}12 \\
22 \\
15 \\
\vdots \\
\vdots\end{array}$ & $\begin{array}{r}50 \\
20 \\
1 \\
1 \\
1\end{array}$ & $\begin{array}{r}10 \\
30 \\
20 \\
L \\
2\end{array}$ & $\begin{array}{r}L \\
L \\
L \\
N \\
20\end{array}$ & $\begin{array}{l}N \\
N \\
N \\
N \\
N\end{array}$ \\
\hline $\begin{array}{l}288-V \\
289-5 \\
290-5 \\
291-5 \\
292-5\end{array}$ & $\begin{array}{l}3 \\
3 \\
3 \\
2 \\
1.5\end{array}$ & $\begin{array}{l}2 \\
1.5 \\
1.5 \\
1.5 \\
.7\end{array}$ & $\begin{array}{l}.3 \\
1 \\
.7 \\
.7\end{array}$ & $\begin{array}{l}.3 \\
.3 \\
.5 \\
.2 \\
.1\end{array}$ & $\begin{array}{l}200 \\
300 \\
500 \\
200 \\
200\end{array}$ & $\begin{array}{r}.2 \\
.3 \\
.2 \\
.3 \\
L(.2)\end{array}$ & $\begin{array}{c}G \\
10 \\
L \\
L \\
L\end{array}$ & $\begin{array}{l}500 \\
500 \\
500 \\
500 \\
500\end{array}$ & $\begin{array}{l}I \\
L \\
L \\
L \\
L\end{array}$ & $\begin{array}{l}5 \\
7 \\
7 \\
15\end{array}$ & $\begin{array}{l}5 \\
\vdots \\
5 \\
5 \\
5\end{array}$ & $\begin{array}{l}12 \\
15 \\
12 \\
2 \\
1\end{array}$ & $\begin{array}{l}L \\
L \\
L \\
i \\
V\end{array}$ & $\begin{array}{l}\text { N } \\
L \\
L \\
L \\
N\end{array}$ \\
\hline $\begin{array}{l}293-5 \\
294-5 \\
295-5 \\
296-5 \\
297-5\end{array}$ & $\begin{array}{l}1.5 \\
1 \\
2 \\
5 \\
5\end{array}$ & $\begin{array}{l}.7 \\
1.5 \\
1.7 \\
1\end{array}$ & $\begin{array}{r}.7 \\
.7 \\
1.5 \\
1.5 \\
.1 .5\end{array}$ & $\begin{array}{l}.1 \\
.3 \\
.2 \\
.5 \\
.5\end{array}$ & $\begin{array}{l}200 \\
200 \\
500 \\
500 \\
500\end{array}$ & $\begin{array}{l}.2 \\
.2 \\
\mathrm{~N} \\
\mathrm{~N} \\
\mathrm{~N}\end{array}$ & $\begin{array}{l}L \\
L \\
N \\
N \\
N\end{array}$ & $\begin{array}{r}500 \\
500 \\
1,000 \\
1,000 \\
1,000\end{array}$ & $\begin{array}{l}L \\
L \\
1 \\
L \\
L\end{array}$ & $\begin{array}{r}\mathrm{L} \\
7 \\
\mathrm{~N} \\
15 \\
15\end{array}$ & $\begin{array}{r}5 \\
5 \\
5 \\
70 \\
50\end{array}$ & $\begin{array}{r}L \\
L \\
50 \\
50 \\
70\end{array}$ & $\begin{array}{r}N \\
N \\
50 \\
N \\
N\end{array}$ & $\begin{array}{r}\mathrm{N} \\
10 \\
\mathrm{~N} \\
\mathrm{~N} \\
\mathrm{~N}\end{array}$ \\
\hline $\begin{array}{l}298-5 \\
299-50 \\
300-5 \\
301-5 \\
302-5\end{array}$ & $\begin{array}{l}7 \\
10 \\
2 \\
2 \\
1.5\end{array}$ & $\begin{array}{l}2 \\
3 \\
.7 \\
.7 \\
.5\end{array}$ & $\begin{array}{l}2 \\
2 \\
1.5 \\
1.5 \\
1.5\end{array}$ & $\begin{array}{l}.7 \\
.7 \\
.3 \\
.2 \\
.2\end{array}$ & $\begin{array}{r}700 \\
1,000 \\
300 \\
300 \\
200\end{array}$ & $\begin{array}{l}\mathrm{N} \\
\mathrm{N} \\
\mathrm{N} \\
\mathrm{N} \\
\mathrm{N}\end{array}$ & $\begin{array}{l}20 \\
N \\
N \\
N \\
N\end{array}$ & $\begin{array}{l}1,000 \\
1,500 \\
1,000 \\
700 \\
1.000\end{array}$ & $\begin{array}{l}L \\
L \\
L \\
i .5\end{array}$ & $\begin{array}{r}15 \\
20 \\
N \\
L \\
N\end{array}$ & $\begin{array}{r}100 \\
150 \\
10 \\
15 \\
N\end{array}$ & $\begin{array}{r}50 \\
100 \\
30 \\
30 \\
30\end{array}$ & $\begin{array}{r}N \\
50 \\
N \\
50 \\
N\end{array}$ & $\begin{array}{l}\text { N } \\
\text { L } \\
\text { N } \\
5 \\
\text { L }\end{array}$ \\
\hline $\begin{array}{l}303-5 \\
304-5 \\
305-5 \\
306-5 \\
307-50\end{array}$ & $\begin{array}{l}5 \\
2 \\
3 \\
1.5 \\
10\end{array}$ & $2_{2^{1} .7}^{.7}$ & $\begin{array}{l}2 \\
1.5 \\
2 \\
1 \\
1.5\end{array}$ & $\begin{array}{l}.5 \\
.2 \\
.5 \\
.3 \\
.7\end{array}$ & $\begin{array}{l}700 \\
500 \\
500 \\
500 \\
700\end{array}$ & $\begin{array}{l}\mathrm{N} \\
\mathrm{N} \\
\mathrm{N} \\
\mathrm{N} \\
\mathrm{N}\end{array}$ & $\begin{array}{r}20 \\
N \\
20 \\
20 \\
N\end{array}$ & $\begin{array}{l}1,000 \\
1,500 \\
1,000 \\
1,500 \\
1,000\end{array}$ & $\begin{array}{l}1 \\
1 \\
1 \\
1.5 \\
L\end{array}$ & $\begin{array}{l}15 \\
10 \\
10 \\
10 \\
15\end{array}$ & $\begin{array}{r}30 \\
N \\
10 \\
5 \\
50\end{array}$ & $\begin{array}{l}50 \\
30 \\
50 \\
50 \\
70\end{array}$ & $\begin{array}{r}30 \\
N \\
50 \\
N \\
100\end{array}$ & $\begin{array}{r}10 \\
N \\
N \\
N \\
20\end{array}$ \\
\hline $\begin{array}{l}308-50 \\
309-R \\
310-5 \\
311-5 \\
312-V\end{array}$ & $\begin{array}{r}7 \\
7 \\
10 \\
10 \\
7\end{array}$ & $\begin{array}{l}2 \\
1.5 \\
1.5 \\
1.5 \\
.2\end{array}$ & $\begin{array}{l}1.5 \\
2 \\
2 \\
2 \\
.5\end{array}$ & $\begin{array}{l}.7 \\
.5 \\
.5 \\
.5 \\
.1\end{array}$ & $\begin{array}{r}1,000 \\
500 \\
700 \\
1,000 \\
100\end{array}$ & $\begin{array}{l}N \\
N \\
N \\
N \\
N\end{array}$ & $\begin{array}{r}N \\
N \\
N \\
20 \\
100\end{array}$ & $\begin{array}{l}1,000 \\
1,000 \\
2,000 \\
2,000 \\
1,500\end{array}$ & $\begin{array}{l}1 \\
1 \\
1 \\
1 \\
1\end{array}$ & $\begin{array}{l}15 \\
15 \\
15 \\
15 \\
N\end{array}$ & $\begin{array}{r}100 \\
20 \\
50 \\
100 \\
10\end{array}$ & $\begin{array}{r}100 \\
50 \\
50 \\
50 \\
30\end{array}$ & $\begin{array}{r}100 \\
N \\
30 \\
30 \\
N\end{array}$ & $\begin{array}{r}\text { L } \\
\text { L } \\
\text { L } \\
10 \\
200\end{array}$ \\
\hline $\begin{array}{l}313-R \\
314-V \\
315-A \\
316 a-A \\
316 b-A\end{array}$ & $\begin{array}{l}3 \\
.1 \\
1.5 \\
1.5 \\
1\end{array}$ & $\begin{array}{l}.05 \\
.02 \\
.3 \\
.7 \\
.7\end{array}$ & $\begin{array}{l}7 \\
.1 \\
.7 \\
1.5\end{array}$ & $\begin{array}{l}.02 \\
.01 \\
.1 \\
.2 \\
.2\end{array}$ & $\begin{array}{r}G(5,000) \\
30 \\
200 \\
300 \\
1,000\end{array}$ & $\begin{array}{l}\mathrm{N} \\
\mathrm{N} \\
\mathrm{N} \\
\mathrm{L} \\
.7\end{array}$ & $\begin{array}{r}L \\
L \\
20 \\
L \\
L\end{array}$ & $\begin{array}{r}N \\
100 \\
300 \\
500 \\
500\end{array}$ & $\begin{array}{l}N \\
N \\
L \\
L \\
L\end{array}$ & $\begin{array}{l}N \\
N \\
5 \\
N \\
L\end{array}$ & $\begin{array}{l}N \\
L \\
L \\
7 \\
5\end{array}$ & $\begin{array}{r}10 \\
5 \\
10 \\
7 \\
7\end{array}$ & $\begin{array}{r}N \\
N \\
30 \\
30 \\
30\end{array}$ & $\begin{array}{r}N \\
N \\
10 \\
L \\
N\end{array}$ \\
\hline $\begin{array}{l}316 c-A \\
316 d-A \\
316 e-A \\
316 f-A \\
316 g-A\end{array}$ & $\begin{array}{r}.5 \\
1.5 \\
1.5 \\
.5 \\
.3\end{array}$ & $\begin{array}{l}.5 \\
.5 \\
.7 \\
.15 \\
.1\end{array}$ & $\begin{array}{l}1 \\
.7 \\
.7 \\
.7 \\
.7\end{array}$ & $\begin{array}{l}.07 \\
.1 \\
.15 \\
.05 \\
.05\end{array}$ & $\begin{array}{l}500 \\
200 \\
500 \\
150 \\
150\end{array}$ & $\begin{array}{l}\mathrm{L} \\
\mathrm{N} \\
\mathrm{N} \\
\mathrm{N} \\
\mathrm{N}\end{array}$ & $\begin{array}{l}\mathrm{L} \\
\mathrm{L} \\
\mathrm{L} \\
\mathrm{L} \\
\mathrm{L}\end{array}$ & $\begin{array}{l}500 \\
300 \\
500 \\
500 \\
500\end{array}$ & $\begin{array}{l}L \\
L \\
L \\
L \\
L\end{array}$ & $\begin{array}{l}N \\
L \\
N \\
N \\
N\end{array}$ & $\begin{array}{l}\mathrm{L} \\
\mathrm{L} \\
\mathrm{L} \\
\mathrm{L} \\
\mathrm{L}\end{array}$ & $\begin{array}{r}5 \\
7 \\
7 \\
10 \\
L\end{array}$ & $\begin{array}{l}20 \\
30 \\
30 \\
30 \\
30\end{array}$ & $\begin{array}{l}7 \\
N \\
N \\
7 \\
7\end{array}$ \\
\hline $\begin{array}{l}316 h-A \\
316 i-A \\
316 j-V \\
317-V \\
318-S\end{array}$ & $\begin{array}{l}L^{2} .3 \\
3 \\
1.5\end{array}$ & $\begin{array}{r}.3 \\
.3 \\
.5 \\
.5\end{array}$ & $\begin{array}{l}1 \\
1 \\
.05 \\
.7 \\
1.5\end{array}$ & $\begin{array}{l}.2 \\
.1 \\
.015 \\
.15 \\
.3\end{array}$ & $\begin{array}{r}200 \\
300 \\
15 \\
150 \\
500\end{array}$ & $\begin{array}{l}L \\
L \\
N \\
N \\
N\end{array}$ & $\begin{array}{c}\mathrm{L} \\
\mathrm{L} \\
\mathrm{L} \\
10 \\
\mathrm{~L}\end{array}$ & $\begin{array}{r}300 \\
300 \\
30 \\
300 \\
200\end{array}$ & $\begin{array}{l}L \\
L \\
N \\
L\end{array}$ & $\begin{array}{l}\mathrm{L} \\
5 \\
\mathrm{~N} \\
5 \\
5\end{array}$ & $\begin{array}{r}7 \\
L \\
L \\
20 \\
L\end{array}$ & $\begin{array}{r}7 \\
10 \\
1 \\
20 \\
10\end{array}$ & $\begin{array}{r}20 \\
20 \\
\mathrm{~N} \\
\mathrm{~N} \\
20\end{array}$ & $\begin{array}{r}5 \\
15 \\
N \\
10 \\
N\end{array}$ \\
\hline $\begin{array}{l}319-S \\
320-S \\
321-S \\
322-R \\
323-S\end{array}$ & $\begin{array}{l}2 \\
1.5 \\
1 \\
2 \\
1\end{array}$ & $\begin{array}{l}.7 \\
.7 \\
.7 \\
.7 \\
.7\end{array}$ & $\begin{array}{l}1 \\
1.5 \\
1 \\
1.5 \\
1.5\end{array}$ & $\begin{array}{l}.3 \\
.2 \\
.3 \\
.3 \\
.3\end{array}$ & $\begin{array}{l}300 \\
200 \\
300 \\
700 \\
500\end{array}$ & $\begin{array}{l}\mathrm{N} \\
\mathrm{N} \\
\mathrm{N} \\
\mathrm{N} \\
\mathrm{N}\end{array}$ & $\begin{array}{l}L \\
L \\
L \\
L \\
L\end{array}$ & $\begin{array}{l}200 \\
200 \\
200 \\
300 \\
300\end{array}$ & $\begin{array}{l}L \\
1 \\
L \\
L \\
1\end{array}$ & $\begin{array}{l}5 \\
L \\
5 \\
7 \\
5\end{array}$ & $\begin{array}{l}L \\
L \\
L \\
L\end{array}$ & $\begin{array}{l}10 \\
10 \\
10 \\
20 \\
20\end{array}$ & $\begin{array}{l}30 \\
30 \\
30 \\
50 \\
30\end{array}$ & $\begin{array}{l}\mathrm{L} \\
\mathrm{N} \\
\mathrm{N} \\
\mathrm{N} \\
\mathrm{N}\end{array}$ \\
\hline $\begin{array}{l}324-S \\
325-A \\
326-R \\
327-A \\
328-R\end{array}$ & $\begin{array}{l}2^{.3} \\
3 \\
1.5\end{array}$ & $\begin{array}{r}.2 \\
.2 \\
1.5 \\
.2 \\
1.5\end{array}$ & $\begin{array}{l}1.5 \\
1.5 \\
.3\end{array}$ & $\begin{array}{l}.7 \\
.3 \\
.7 \\
.1 \\
.3\end{array}$ & $\begin{array}{l}150 \\
300 \\
700 \\
200 \\
700\end{array}$ & $\begin{array}{l}\mathrm{N} \\
\mathrm{L} \\
\mathrm{N} \\
{ }_{\mathrm{N}}\end{array}$ & $\begin{array}{r}L \\
10 \\
L \\
10 \\
L\end{array}$ & $\begin{array}{l}300 \\
300 \\
700 \\
300 \\
700\end{array}$ & $\begin{array}{l}1 \\
1.5 \\
1 \\
1 \\
1\end{array}$ & $\begin{array}{r}\mathrm{L} \\
7 \\
15 \\
5 \\
15\end{array}$ & $\begin{array}{r}\mathrm{L} \\
20 \\
100 \\
L \\
L\end{array}$ & $\begin{array}{r}15 \\
30 \\
70 \\
1,500 \\
30\end{array}$ & $\begin{array}{r}N \\
50 \\
50 \\
30 \\
30\end{array}$ & $\begin{array}{l}N \\
N \\
5 \\
5 \\
N\end{array}$ \\
\hline $\begin{array}{l}329-R \\
330-S \\
331-R \\
332-A \\
333-S\end{array}$ & $\begin{array}{l}1 \\
3 \\
1.5 \\
.5\end{array}$ & $\begin{array}{r}.2 \\
.2 \\
.7 \\
.5\end{array}$ & $2^{1} .7$ & $\begin{array}{l}.1 \\
.1 \\
.5 \\
.07 \\
.2\end{array}$ & $\begin{array}{r}300 \\
300 \\
1,000 \\
300 \\
200\end{array}$ & $\begin{array}{l}N \\
N \\
N \\
N\end{array}$ & $\begin{array}{l}\mathrm{L} \\
\mathrm{L} \\
\mathrm{L} \\
\mathrm{L} \\
\mathrm{L}\end{array}$ & $\begin{array}{l}200 \\
300 \\
200 \\
700 \\
200\end{array}$ & $\begin{array}{l}1 \\
1 \\
N \\
L \\
1\end{array}$ & $\begin{array}{c}\mathrm{N} \\
\mathrm{L} \\
30 \\
\mathrm{~N} \\
\mathrm{~L}\end{array}$ & $\begin{array}{c}\mathrm{L} \\
\mathrm{L} \\
10 \\
\mathrm{~L} \\
\mathrm{~L}\end{array}$ & $\begin{array}{l}15 \\
15 \\
30 \\
30 \\
15\end{array}$ & $\begin{array}{l}L \\
L \\
N \\
30 \\
20\end{array}$ & $\begin{array}{l}N \\
N \\
N \\
L \\
N\end{array}$ \\
\hline
\end{tabular}




\section{Basin primitive area, California-Continued}

\begin{tabular}{|c|c|c|c|c|c|c|c|c|}
\hline \multirow{2}{*}{$\begin{array}{l}\text { Sample } \\
\text { and } \\
\text { type }\end{array}$} & \multicolumn{6}{|c|}{$\begin{array}{l}\text { Semiquantitative spectrographic } \\
\text { analyses }(\mathrm{ppm})-\text {-Cont inued }\end{array}$} & $\begin{array}{c}\text { Chemical analyses } \\
(\mathrm{ppm})\end{array}$ & \multirow{2}{*}{ Remarks } \\
\hline & $\begin{array}{ll}\mathrm{Nb} & \mathrm{Ni} \\
(10) & (2)\end{array}$ & $\begin{array}{ccc}\mathrm{Pb} & \mathrm{Sc} & \mathrm{Sr} \\
(10) & (5) & (50)\end{array}$ & $\begin{array}{l}V \\
(10)(50)\end{array}$ & $\begin{array}{l}Y \\
(5)\end{array}$ & $\begin{array}{l}\mathrm{Zn} \\
(200)\end{array}$ & $\begin{array}{l}\mathrm{Zr} \\
(10)\end{array}$ & $\begin{array}{ccc}\mathrm{Au}^{2} & \mathrm{Hg}^{3} & \mathrm{u}^{4} \\
(0.02) & (0.01) & (20)\end{array}$ & \\
\hline
\end{tabular}

\begin{tabular}{|c|c|c|c|c|c|c|c|c|c|c|c|c|c|c|}
\hline $\begin{array}{l}284-5 \\
285-5 \\
286-5 \\
287-5 \\
287 a-A\end{array}$ & $\begin{array}{r}\mathrm{L} \\
10 \\
\mathrm{~L} \\
\mathrm{~L} \\
\mathrm{~L}\end{array}$ & $\begin{array}{r}7 \\
50 \\
20 \\
5 \\
5\end{array}$ & $\begin{array}{r}30 \\
20 \\
20 \\
15 \\
100\end{array}$ & $\begin{array}{r}5 \\
20 \\
15 \\
5 \\
15\end{array}$ & $\begin{array}{l}500 \\
700 \\
500 \\
300 \\
300\end{array}$ & $\begin{array}{r}50 \\
200 \\
100 \\
30 \\
50\end{array}$ & $\begin{array}{l}N \\
N \\
N \\
N \\
N\end{array}$ & $\begin{array}{r}L \\
15 \\
15 \\
5 \\
10\end{array}$ & $\begin{array}{l}273 \\
281 \\
281 \\
251 \\
297\end{array}$ & $\begin{array}{r}100 \\
200 \\
150 \\
50 \\
100\end{array}$ & $\begin{array}{l}L \\
L \\
L \\
L \\
0.02\end{array}$ & $\begin{array}{l}1 \\
0.04 \\
.03 \\
.03 \\
.01\end{array}$ & $\begin{array}{l}L \\
L \\
L \\
L \\
L\end{array}$ & $\begin{array}{l}--- \\
--- \\
--- \\
--\end{array}$ \\
\hline $\begin{array}{l}288-V \\
289-S \\
290-S \\
291-S \\
292-S\end{array}$ & $\begin{array}{l}15 \\
10 \\
15 \\
L \\
L\end{array}$ & $\begin{array}{l}5 \\
5 \\
5 \\
7 \\
5\end{array}$ & $\begin{array}{l}20 \\
20 \\
15 \\
20 \\
20\end{array}$ & $\begin{array}{l}7 \\
5 \\
7 \\
7 \\
L\end{array}$ & $\begin{array}{r}N \\
500 \\
500 \\
300 \\
300\end{array}$ & $\begin{array}{l}50 \\
30 \\
50 \\
50 \\
15\end{array}$ & $\begin{array}{l}N \\
N \\
N \\
N \\
N\end{array}$ & $\begin{array}{l}10 \\
15 \\
15 \\
7 \\
L\end{array}$ & $\begin{array}{c}2 L(25) \\
250 \\
252 \\
255 \\
242\end{array}$ & $\begin{array}{r}150 \\
100 \\
200 \\
50 \\
70\end{array}$ & $\mathrm{~L}_{\mathrm{L}}^{\mathrm{L}} .02$ & $\begin{array}{l}.01 \\
.02 \\
.01 \\
.01 \\
.01\end{array}$ & $\begin{array}{l}L \\
L \\
L \\
L \\
L\end{array}$ & Alt. grn; $B, G=2,000 \mathrm{ppm}$. \\
\hline $\begin{array}{l}293-5 \\
294-5 \\
295-5 \\
296-5 \\
297-5\end{array}$ & $\begin{array}{l}\mathrm{L} \\
\mathrm{L} \\
\mathrm{L} \\
\mathrm{L} \\
\mathrm{L}\end{array}$ & $\begin{array}{r}5 \\
7 \\
15 \\
30 \\
30\end{array}$ & $\begin{array}{l}15 \\
20 \\
20 \\
20 \\
20\end{array}$ & $\begin{array}{r}L \\
5 \\
5 \\
10 \\
10\end{array}$ & $\begin{array}{r}300 \\
300 \\
700 \\
1,500 \\
1,000\end{array}$ & $\begin{array}{r}20 \\
30 \\
50 \\
150 \\
100\end{array}$ & $\begin{array}{l}N \\
N \\
N \\
N \\
N\end{array}$ & $\begin{array}{r}5 \\
15 \\
10 \\
10 \\
5\end{array}$ & $\begin{array}{l}228 \\
240 \\
244 \\
235 \\
240\end{array}$ & $\begin{array}{r}70 \\
70 \\
100 \\
150 \\
150\end{array}$ & $\begin{array}{r}\mathrm{L} \\
.02 \\
.08 \\
.04\end{array}$ & $\begin{array}{l}\mathrm{L} \\
.01 \\
.02 \\
.02\end{array}$ & $\begin{array}{l}\mathrm{L} \\
\mathrm{L} \\
\mathrm{L} \\
\mathrm{L}\end{array}$ & Pothole sediment. \\
\hline $\begin{array}{l}298-5 \\
299-50 \\
300-5 \\
301-5 \\
302-5\end{array}$ & $\begin{array}{l}\mathrm{L} \\
\mathrm{L} \\
\mathrm{L} \\
\mathrm{L} \\
\mathrm{L}\end{array}$ & $\begin{array}{l}50 \\
50 \\
20 \\
20 \\
15\end{array}$ & $\begin{array}{l}20 \\
50 \\
20 \\
20 \\
20\end{array}$ & $\begin{array}{r}15 \\
30 \\
5 \\
5 \\
N\end{array}$ & $\begin{array}{r}1,500 \\
1,500 \\
700 \\
1,000 \\
1,000\end{array}$ & $\begin{array}{r}150 \\
200 \\
50 \\
70 \\
50\end{array}$ & $\begin{array}{l}N \\
N \\
N \\
N \\
N\end{array}$ & $\begin{array}{r}10 \\
20 \\
N \\
10 \\
N\end{array}$ & $\begin{array}{l}250 \\
278 \\
220 \\
250 \\
229\end{array}$ & $\begin{array}{r}200 \\
150 \\
100 \\
100 \\
50\end{array}$ & $\begin{array}{l}\mathrm{L} \\
.02 \\
.04 \\
.2\end{array}$ & $\begin{array}{l}.01 \\
.02 \\
.03 \\
.03 \\
.03\end{array}$ & $\begin{array}{l}\mathrm{L} \\
\mathrm{L} \\
\mathrm{L} \\
\mathrm{L} \\
\mathrm{L}\end{array}$ & Vol, ridge soil. \\
\hline $\begin{array}{l}303-5 \\
304-5 \\
305-5 \\
306-5 \\
307-50\end{array}$ & $\begin{array}{l}L \\
L \\
L \\
15\end{array}$ & $\begin{array}{l}30 \\
15 \\
20 \\
15 \\
30\end{array}$ & $\begin{array}{l}20 \\
50 \\
30 \\
30 \\
20\end{array}$ & $\begin{array}{r}10 \\
N \\
10 \\
7 \\
30\end{array}$ & $\begin{array}{l}1,500 \\
1,500 \\
1,000 \\
1,000 \\
1,000\end{array}$ & $\begin{array}{r}100 \\
30 \\
100 \\
50 \\
200\end{array}$ & $\begin{array}{l}N \\
N \\
N \\
N \\
N\end{array}$ & $\begin{array}{r}10 \\
10 \\
10 \\
N \\
30\end{array}$ & $\begin{array}{r}257 \\
249 \\
2110 \\
245 \\
260\end{array}$ & $\begin{array}{l}150 \\
150 \\
100 \\
100 \\
500\end{array}$ & $\begin{array}{l}L \\
L \\
L \\
L \\
L\end{array}$ & $\begin{array}{l}.01 \\
.01 \\
.04 \\
.03 \\
.01\end{array}$ & $\begin{array}{l}L \\
L \\
L \\
L \\
L\end{array}$ & Vol, soil above $\mathrm{G}$ contact. \\
\hline $\begin{array}{l}308-S o \\
309-R \\
310-5 \\
311-5 \\
312-V\end{array}$ & $\begin{array}{r}15 \\
20 \\
15 \\
10 \\
1\end{array}$ & $\begin{array}{l}50 \\
20 \\
20 \\
50 \\
30\end{array}$ & $\begin{array}{l}50 \\
30 \\
20 \\
50 \\
10\end{array}$ & $\begin{array}{r}30 \\
20 \\
15 \\
15 \\
7\end{array}$ & $\begin{array}{r}500 \\
700 \\
700 \\
3,000 \\
150\end{array}$ & $\begin{array}{l}200 \\
100 \\
150 \\
100 \\
150\end{array}$ & $\begin{array}{l}N \\
N \\
N \\
N \\
N\end{array}$ & $\begin{array}{l}20 \\
50 \\
20 \\
20 \\
10\end{array}$ & $\begin{array}{r}270 \\
260 \\
235 \\
265 \\
2130\end{array}$ & $\begin{array}{l}200 \\
500 \\
200 \\
150 \\
100\end{array}$ & $\begin{array}{l}L \\
L \\
L \\
L\end{array}$ & $\begin{array}{l}.02 \\
.01 \\
.03 \\
1.3\end{array}$ & $\begin{array}{r}L \\
L \\
L \\
600\end{array}$ & $\begin{array}{l}\text { Vol, soil. } \\
\text { Grn. } \\
\text { (15). }\end{array}$ \\
\hline $\begin{array}{l}313-R \\
314-V \\
315-A \\
316 a-A \\
316 b-A\end{array}$ & $\begin{array}{r}N \\
L \\
10 \\
N \\
10\end{array}$ & $\begin{array}{l}N \\
L \\
5 \\
L \\
L\end{array}$ & $\begin{array}{l}N \\
15 \\
30 \\
20 \\
50\end{array}$ & $\begin{array}{r}N \\
N \\
5 \\
10 \\
10\end{array}$ & $\begin{array}{r}N \\
N \\
200 \\
300 \\
300\end{array}$ & $\begin{array}{r}300 \\
1 . \\
30 \\
100 \\
50\end{array}$ & $\begin{array}{l}\mathrm{N} \\
\mathrm{N} \\
\mathrm{N} \\
\mathrm{N} \\
\mathrm{N}\end{array}$ & $\begin{array}{r}10 \\
\mathrm{~N} \\
20 \\
15 \\
10\end{array}$ & $\begin{array}{l}N \\
N \\
N \\
N \\
N\end{array}$ & $\begin{array}{r}N \\
N \\
70 \\
150 \\
150\end{array}$ & $\begin{array}{l}\mathrm{N} \\
\mathrm{N} \\
\mathrm{N} \\
\mathrm{N} \\
\mathrm{N}\end{array}$ & $\begin{array}{l}.11 \\
.08 \\
.07 \\
.10 \\
.08\end{array}$ & $\begin{array}{l}\mathrm{N} \\
\mathrm{N} \\
\mathrm{N} \\
\mathrm{N} \\
\mathrm{N}\end{array}$ & $\begin{array}{l}\text { Met, tactite } \\
\text { White quartz, pyritized grn. } \\
\text { Grn, Fe stain. } \\
\qquad \begin{array}{l}\text { Do. } \\
\text { Do. }\end{array}\end{array}$ \\
\hline $\begin{array}{l}316 \mathrm{c}-\mathrm{A} \\
316 \mathrm{~d}-\mathrm{A} \\
316 \mathrm{e}-\mathrm{A} \\
316 \mathrm{f}-\mathrm{A} \\
316 \mathrm{~g}-\mathrm{A}\end{array}$ & $\begin{array}{r}N \\
L \\
L \\
10 \\
L\end{array}$ & $\begin{array}{l}\mathrm{L} \\
\mathrm{L} \\
\mathrm{L} \\
\mathrm{L} \\
\mathrm{L}\end{array}$ & $\begin{array}{l}50 \\
15 \\
15 \\
15 \\
15\end{array}$ & $\begin{array}{l}\mathrm{L} \\
7 \\
7 \\
\mathrm{~N} \\
\mathrm{~L}\end{array}$ & $\begin{array}{l}300 \\
300 \\
300 \\
300 \\
150\end{array}$ & $\begin{array}{l}30 \\
30 \\
30 \\
10 \\
15\end{array}$ & $\begin{array}{l}\mathrm{N} \\
\mathrm{N} \\
\mathrm{N} \\
\mathrm{N} \\
\mathrm{N}\end{array}$ & $\begin{array}{r}10 \\
10 \\
10 \\
L \\
L\end{array}$ & $\begin{array}{l}N \\
N \\
N \\
N \\
N\end{array}$ & $\begin{array}{r}150 \\
100 \\
150 \\
70 \\
100\end{array}$ & $\begin{array}{l}\mathrm{N} \\
\mathrm{N} \\
\mathrm{N} \\
\mathrm{N} \\
\mathrm{N}\end{array}$ & $\begin{array}{l}.10 \\
.20 \\
.09 \\
.06 \\
.06\end{array}$ & $\begin{array}{l}N \\
N \\
N \\
N \\
N\end{array}$ & $\begin{array}{l}\text { Do. } \\
\text { Do. } \\
\text { Do. } \\
\text { Do. } \\
\text { Do. }\end{array}$ \\
\hline $\begin{array}{l}316 h-A \\
316 i-A \\
316 j-V \\
317-V \\
318-S\end{array}$ & $\begin{array}{c}10 \\
L \\
N \\
N \\
15\end{array}$ & $\begin{array}{r}L \\
L \\
L \\
10 \\
L\end{array}$ & $\begin{array}{r}70 \\
30 \\
N \\
10 \\
15\end{array}$ & $\begin{array}{r}7 \\
5 \\
N \\
7 \\
10\end{array}$ & $\begin{array}{r}300 \\
300 \\
N \\
300 \\
.300\end{array}$ & $\begin{array}{r}70 \\
30 \\
L \\
100 \\
50\end{array}$ & $\begin{array}{r}\mathrm{N} \\
\mathrm{N} \\
\mathrm{N} \\
\mathrm{N} \\
\mathrm{N}\end{array}$ & $\begin{array}{r}10 \\
10 \\
N \\
L \\
20\end{array}$ & $\begin{array}{l}\mathrm{N} \\
\mathrm{N} \\
\mathrm{N} \\
\mathrm{N} \\
\mathrm{N}\end{array}$ & $\begin{array}{r}100 \\
100 \\
N \\
100 \\
300\end{array}$ & $\begin{array}{l}N \\
N \\
N \\
N \\
N\end{array}$ & $\begin{array}{l}.12 \\
.08 \\
.06 \\
.10 \\
.06\end{array}$ & $\begin{array}{l}N \\
N \\
N \\
N \\
N\end{array}$ & $\begin{aligned} & \text { Do. } \\
& \text { Do. } \\
& \text { Do. } \\
& \text { Fault gouge zone. }\end{aligned}$ \\
\hline $\begin{array}{l}319-5 \\
320-S \\
321-S \\
322-R \\
323-5\end{array}$ & $\begin{array}{r}15 \\
N \\
20 \\
20 \\
15\end{array}$ & $\begin{array}{l}5 \\
5 \\
L \\
L \\
5\end{array}$ & $\begin{array}{l}15 \\
15 \\
15 \\
15 \\
15\end{array}$ & $\begin{array}{r}7 \\
7 \\
7 \\
10 \\
7\end{array}$ & $\begin{array}{l}300 \\
300 \\
300 \\
300 \\
300\end{array}$ & $\begin{array}{r}70 \\
50 \\
70 \\
100 \\
70\end{array}$ & $\begin{array}{l}N \\
N \\
N \\
N \\
N\end{array}$ & $\begin{array}{l}20 \\
15 \\
15 \\
10 \\
20\end{array}$ & $\begin{array}{l}N \\
N \\
N \\
N \\
N\end{array}$ & $\begin{array}{r}150 \\
150 \\
200 \\
50 \\
200\end{array}$ & $\begin{array}{l}N \\
N \\
N \\
N \\
N\end{array}$ & $\begin{array}{l}.06 \\
.08 \\
.06 \\
.13 \\
.07\end{array}$ & $\begin{array}{l}N \\
N \\
-- \\
N \\
N\end{array}$ & $\mathrm{Grn}$. \\
\hline $\begin{array}{l}324-S \\
325-A \\
326-R \\
327-A \\
328-R\end{array}$ & $\begin{array}{r}N \\
15 \\
20 \\
L \\
N\end{array}$ & $\begin{array}{r}\mathrm{L} \\
20 \\
100 \\
10 \\
\mathrm{~L}\end{array}$ & $\begin{array}{l}10 \\
15 \\
20 \\
15 \\
15\end{array}$ & $\begin{array}{r}5 \\
7 \\
15 \\
5 \\
10\end{array}$ & $\begin{array}{l}300 \\
150 \\
300 \\
150 \\
700\end{array}$ & $\begin{array}{r}30 \\
100 \\
200 \\
30 \\
200\end{array}$ & $\begin{array}{l}N \\
N \\
N \\
N \\
N\end{array}$ & $\begin{array}{r}L \\
15 \\
20 \\
L \\
15\end{array}$ & $\begin{array}{l}N \\
N \\
N \\
N \\
N\end{array}$ & $\begin{array}{r}30 \\
300 \\
300 \\
100 \\
150\end{array}$ & $\begin{array}{l}\mathrm{N} \\
\mathrm{N} \\
\mathrm{N} \\
\mathrm{N}^{.04}\end{array}$ & $\begin{array}{l}.03 \\
.20 \\
.26 \\
.12 \\
.21\end{array}$ & $\begin{array}{l}\mathrm{N} \\
\mathrm{N} \\
\mathrm{N} \\
\mathrm{N} \\
\mathrm{N}\end{array}$ & $\begin{array}{l}B i=L . \\
\text { Vol, Fe stain. } \\
\text { Vol. } \\
\text { Vol, Fe stain. } \\
\text { Vol, andesite dike. }\end{array}$ \\
\hline $\begin{array}{l}329-R \\
330-S \\
331-R \\
332-4 \\
333-S\end{array}$ & $\begin{array}{r}10 \\
\mathrm{~L} \\
\mathrm{~N} \\
\mathrm{~N} \\
10\end{array}$ & $\begin{array}{c}\mathrm{L} \\
\mathrm{L} \\
10 \\
\mathrm{~L} \\
\mathrm{~L}\end{array}$ & $\begin{array}{r}10 \\
15 \\
N \\
10 \\
15\end{array}$ & $\begin{array}{r}7 \\
7 \\
70 \\
5 \\
7\end{array}$ & $\begin{array}{l}300 \\
200 \\
500 \\
300 \\
300\end{array}$ & $\begin{array}{r}15 \\
20 \\
300 \\
20 \\
30\end{array}$ & $\begin{array}{l}N \\
N \\
N \\
N \\
N\end{array}$ & $\begin{array}{r}15 \\
15 \\
20 \\
L \\
15\end{array}$ & $\begin{array}{l}\mathrm{N} \\
\mathrm{N} \\
\mathrm{N} \\
\mathrm{N} \\
\mathrm{N}\end{array}$ & $\begin{array}{r}200 \\
200 \\
70 \\
200 \\
70\end{array}$ & $\begin{array}{l}N \\
N \\
N \\
N \\
N\end{array}$ & $\begin{array}{l}.14 \\
.04 \\
.28 \\
.20 \\
.12\end{array}$ & $\begin{array}{l}\mathrm{N} \\
\mathrm{N} \\
\mathrm{N} \\
\mathrm{N} \\
--\end{array}$ & $\begin{array}{l}\text { Met. } \\
8 i=L . \\
\text { Dio, xenolith. } \\
G r n, \text { Fe stain. } \\
B i=L .\end{array}$ \\
\hline
\end{tabular}




\section{TABLE 4.-Analyses of samples from the Emigrant}

\begin{tabular}{|c|c|c|c|c|c|c|c|c|c|c|c|c|c|c|}
\hline \multirow{2}{*}{$\begin{array}{l}\text { Sample } \\
\text { and } \\
\text { type }\end{array}$} & \multicolumn{4}{|c|}{$\begin{array}{c}\text { Semiquantitative spectrographic } \\
\text { analyses (percent) }\end{array}$} & \multicolumn{10}{|c|}{$\begin{array}{c}\text { Semiquantitative spectrographic } \\
\text { analyses }(\mathrm{ppm}) 1\end{array}$} \\
\hline & $\begin{array}{c}\mathrm{Fe} \\
(0.05)\end{array}$ & $(0.005)$ & $\begin{array}{c}\mathrm{Ca} \\
(0.05)\end{array}$ & $\begin{array}{c}\mathrm{Ti} \\
(0.001)\end{array}$ & $\begin{array}{c}M n \\
(20)\end{array}$ & $\begin{array}{l}\mathrm{Ag} \\
(0.5)\end{array}$ & $\stackrel{B}{(10)}$ & $\begin{array}{c}\mathrm{Ba} \\
(20)\end{array}$ & $\begin{array}{l}\mathrm{Be} \\
\text { (1) }\end{array}$ & $\begin{array}{l}\text { Co } \\
(5)\end{array}$ & $\begin{array}{l}c r \\
(5)\end{array}$ & $\begin{array}{l}\mathrm{Cu} \\
(2)\end{array}$ & $\begin{array}{l}\text { La } \\
(20)\end{array}$ & $\begin{array}{l}\text { Mo } \\
(5)\end{array}$ \\
\hline $334-R$ & $3-$ & 1.5 & 0.3 & 0.3 & 700 & $\mathrm{~N}$ & L & 300 & 1 & 20 & 150 & 30 & 30 & $\mathrm{~N}$ \\
\hline $335-R$ & 3 & 2 & .05 & .3 & 500 & $\mathrm{~N}$ & $i$ & 300 & L & 20 & 150 & 20 & 30 & $\mathrm{~N}$ \\
\hline $336-R$ & 3 & 2 & $L$ & .3 & 300 & N & $\mathrm{L}$ & 300 & L & 20 & 150 & 30 & 50 & $\mathrm{~N}$ \\
\hline $337-R$ & $i$ & 1 & 1.5 & .2 & 700 & $\mathrm{~N}$ & $\mathrm{~L}$ & 70 & $\bar{L}$ & 5 & 30 & 10 & 20 & $\mathrm{~N}$ \\
\hline $338-R$ & .5 & .5 & .7 & .07 & 150 & $\mathrm{~N}$ & $\mathrm{~L}$ & 70 & $i$ & $\hat{L}$ & 5 & 10 & L & N \\
\hline $339-R$ & 3 & 3 & 5 & .2 & 1,500 & $\mathrm{~N}$ & $L$ & 1,000 & l & 7 & 50 & 10 & 30 & $\mathrm{~N}$ \\
\hline $340-R$ & .2 & .3 & 1.5 & .03 & 500 & $\mathrm{~N}$ & $\mathrm{~L}$ & 30 & N & $\mathrm{N}$ & $\mathrm{L}$ & 15 & $\mathrm{~N}$ & $\mathrm{~N}$ \\
\hline $341-R$ & 5 & 1.5 & 10 & .07 & $G(5,000)$ & $\mathrm{N}$ & N & N & $\mathrm{N}$ & 5 & 5 & 70 & $\mathrm{~N}$ & 100 \\
\hline $342-R$ & 3 & .3 & 7 & .07 & 2,000 & $\mathrm{~N}$ & $\mathrm{~N}$ & N & $N$ & $\mathrm{~N}$ & L & 30 & $\mathrm{~N}$ & N \\
\hline $343-A$ & 3 & 1 & 7 & .1 & 2,000 & $\mathrm{~N}$ & $\mathrm{~N}$ & $\mathrm{~N}$ & L & L & $\mathrm{L}$ & 15 & L & $\mathrm{N}$ \\
\hline $344-R$ & 1 & .7 & .7 & .1 & 500 & $\mathrm{~N}$ & $\mathrm{~N}$ & 500 & L & 5 & L & 30 & 20 & $\mathrm{~N}$ \\
\hline $344 b-v$ & 15 & 2 & 2 & .05 & 1,000 & 5 & 15 & 30 & L & N & 7 & 1,500 & 20 & 7 \\
\hline $345-R$ & 2 & $i$ & 1.5 & .2 & 700 & $\mathrm{~N}$ & $\mathrm{~L}$ & 500 & L & 7 & 5 & 20 & 20 & $\mathrm{~N}$ \\
\hline $346-R$ & 3 & 2 & 2 & .3 & 700 & $\mathrm{~N}$ & $i$ & 300 & $\bar{L}$ & 15 & 10 & 30 & L & $\mathrm{N}$ \\
\hline $347-R$ & $i$ & .3 & .5 & .07 & 200 & $\mathrm{~N}$ & $i$ & 500 & L & $L$ & $\mathrm{~L}$ & 15 & 30 & N \\
\hline $348-R$ & 3 & 2 & 1.5 & .3 & 1,000 & $\mathrm{~N}$ & $\mathrm{~L}$ & 500 & L & 15 & 10 & 30 & $\mathrm{~N}$ & $\mathrm{~N}$ \\
\hline $349-R$ & 3 & 1.5 & 1.5 & .3 & 700 & $\mathrm{~N}$ & $\mathrm{~L}$ & 300 & $\bar{L}$ & 15 & $\mathrm{~L}$ & 30 & $\mathrm{~N}$ & $\mathrm{~N}$ \\
\hline $350-R$ & .3 & .2 & 1 & .05 & 300 & $\mathrm{~N}$ & $\mathrm{~L}$ & 50 & 1.5 & N & $\mathrm{L}$ & L & 20 & 5 \\
\hline $351-R$ & 1.5 & .7 & i & .15 & 500 & $\mathrm{~N}$ & $\mathrm{~L}$ & 300 & $\mathrm{~L}$ & 5 & L & 5 & $L$ & 5 \\
\hline $352-R$ & .3 & .15 & .5 & .05 & 200 & N & $\mathrm{L}$ & 300 & 1 & $\mathrm{~N}$ & $\mathrm{~L}$ & $\hat{L}$ & $\mathrm{~N}$ & 5 \\
\hline $353-R$ & 1 & .7 & 1 & .15 & 500 & $\mathrm{~N}$ & L & 500 & 1.5 & 5 & L & L & L & 5 \\
\hline $354-R$ & .5 & .2 & .5 & .05 & 200 & N & $i$ & 300 & $i$ & $\mathrm{~N}$ & $L$ & L & 20 & 5 \\
\hline $355-R$ & 1.5 & 1 & 1 & .15 & 300 & N & $\mathrm{L}$ & 300 & L & 7 & $\mathrm{~L}$ & 5 & 30 & 5 \\
\hline $356-R$ & i & .7 & i & .1 & 200 & N & $i$ & 300 & L & 5 & $\mathrm{~L}$ & 5 & L & 5 \\
\hline $357-R$ & $i$ & .7 & .7 & .1 & 300 & $\mathrm{~N}$ & $\mathrm{~L}$ & 300 & $\mathrm{~L}$ & 5 & $\mathrm{~L}$ & 5 & L & 5 \\
\hline $358-R$ & 1 & .5 & .7 & .1 & 300 & $\mathrm{~N}$ & L & 300 & L & L & L & L & 30 & 5 \\
\hline $359-R$ & 2 & 2 & 2 & .15 & 500 & $\mathrm{~N}$ & $i$ & 150 & $i$ & 15 & 30 & 15 & $\mathrm{~N}$ & 5 \\
\hline $360-R$ & .7 & .3 & .7 & .1 & 300 & $\mathrm{~N}$ & $\mathrm{~L}$ & 500 & $\mathrm{i}$ & $L$ & $\mathrm{~L}$ & L & 30 & 5 \\
\hline $361-R$ & .7 & .3 & .7 & .07 & 300 & $\mathrm{~N}$ & L & 300 & L & $\mathrm{i}$ & $\mathrm{i}$ & $\mathrm{L}$ & 20 & 5 \\
\hline $362-R$ & 1 & .5 & .7 & .1 & 300 & $N$ & L & 500 & L & 5 & L & L & 30 & 5 \\
\hline $363-f$ & .5 & .15 & .3 & .15 & 300 & $\mathrm{~N}$ & L & 300 & L & $\mathrm{N}$ & L & L & 30 & 5 \\
\hline 364. & .7 & .7 & 1 & .15 & 300 & $\mathrm{~N}$ & $L$ & 200 & L & 5 & $L$ & $i$ & 20 & 5 \\
\hline $365-$ & .7 & .5 & .7 & .1 & 300 & $\mathrm{~N}$ & L & 200 & L & ¿ & L & L & L & 5 \\
\hline $366-R$ & .3 & .5 & .7 & .07 & 200 & $\mathrm{~N}$ & L & 300 & L & $i$ & $i$ & 5 & $\mathrm{~N}$ & 5 \\
\hline $367-R$ & .5 & .7 & 1 & .1 & 300 & $\mathrm{~N}$ & L & 300 & L & 5 & L & 5 & L & 5 \\
\hline $368-R$ & .5 & .7. & .7 & .07 & 500 & $\mathrm{~N}$ & $L$ & 300 & L & L & L & 5 & $\mathrm{~N}$ & 5 \\
\hline $369-R$ & .7 & $.7^{\circ}$ & .7 & .1 & 300 & $\mathrm{~N}$ & $\mathrm{~L}$ & 300 & $i$ & 5 & L & 5 & 30 & 5 \\
\hline $370-R$ & 7 & 7 & 10 & .3 & 700 & $\mathrm{~N}$ & 20 & 300 & $\mathrm{~N}$ & 30 & 300 & 7 & $\mathrm{~N}$ & $L(2)$ \\
\hline $371-R$ & 7 & 7 & 10 & .3 & 700 & $\mathrm{~N}$ & L & 70 & 1 & 30 & 1,500 & 15 & 70 & $L(2)$ \\
\hline $372-R$ & 10 & 7 & 10 & .3 & 700 & $\mathrm{~N}$ & $L$ & 70 & $\mathrm{~N}$ & 70 & 1,500 & 10 & $\mathrm{~N}$ & $L(2)$ \\
\hline $\begin{array}{l}373-R \\
374-R\end{array}$ & 10 & G & 1.5 & .15 & 1,500 & $\mathrm{~N}$ & $L$ & 30 & N & 70 & 1,500 & 30 & $\mathrm{~N}$ & $L(2)$ \\
\hline & 7 & G & 10 & .07 & 700 & $\mathrm{~N}$ & L & 150 & N & 30 & 300 & 30 & N & $L(2)$ \\
\hline
\end{tabular}

IIndium was not detected at limit of detection in samples 1-139 and 200-241, and was not looked for in all others. As, Bi, Cd, Sb, and $\mathrm{Sn}$ were not detected at limit of detection in all samples, except as noted in Remarks. Lower limits of detection in ppm: In 100, As 200, Bi 10, Cd 20, Sb $100,5 n 10$.

${ }^{2}$ Atomic adsorption determination.

3 Instrumental, Hg detector determination.

${ }^{4}$ Paper chromatography determination.

${ }^{5}$ Colorometric determination.

${ }^{6}$ CNS wet chemical determination.

${ }^{7} \mathrm{As}, 300 ; \mathrm{Ba}, \mathrm{G}=5,000 ; \mathrm{Bi}, 15 ; \mathrm{Cd}, 500 ; \mathrm{Pb}, \mathrm{G}=20,000 ; \mathrm{Sb}, \mathrm{G}=10,000 \mathrm{ppm}$. Ore, Old Silver Mine shaft.

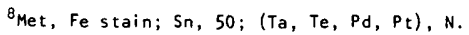

9 Prospect pit, Met; scheelite; Bi, 500; Sn, 10; Cd, 700; (Ta, Te, Pd, Pt), N. 


\section{Basin primitive area, Califormia-Continued}

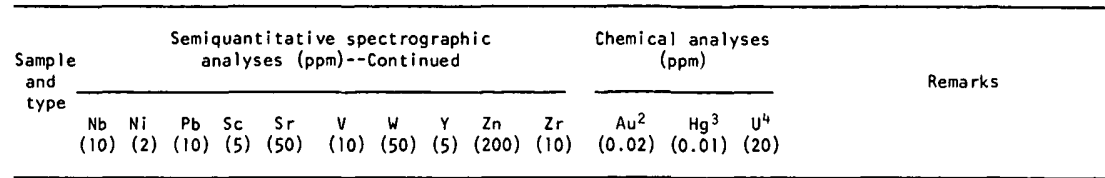

\begin{tabular}{|c|c|c|c|c|c|c|c|c|c|c|c|c|c|c|}
\hline $\begin{array}{l}334-R \\
335-R \\
336-R \\
337-R \\
338-R\end{array}$ & $\begin{array}{r}20 \\
20 \\
20 \\
10 \\
\mathrm{~N}\end{array}$ & $\begin{array}{r}70 \\
100 \\
100 \\
15 \\
7\end{array}$ & $\begin{array}{r}15 \\
15 \\
15 \\
L \\
L\end{array}$ & $\begin{array}{r}20 \\
20 \\
30 \\
5 \\
N\end{array}$ & $\begin{array}{r}150 \\
N \\
N \\
200 \\
L\end{array}$ & $\begin{array}{r}200 \\
200 \\
200 \\
30 \\
15\end{array}$ & $\begin{array}{l}N \\
N \\
N \\
N \\
N\end{array}$ & $\begin{array}{r}30 \\
20 \\
50 \\
15 \\
\mathrm{~L}\end{array}$ & $\begin{array}{l}N \\
N \\
N \\
N \\
N\end{array}$ & $\begin{array}{r}150 \\
200 \\
200 \\
150 \\
70\end{array}$ & $\begin{array}{l}N \\
N \\
N \\
N \\
N\end{array}$ & $\begin{array}{l}0.26 \\
.14 \\
.28 \\
.08 \\
.06\end{array}$ & $\begin{array}{l}N \\
N \\
N \\
N \\
N\end{array}$ & $\begin{array}{l}\text { Dio. } \\
\text { Met, biotite gneiss. } \\
\text { Met, biotite schist. } \\
\text { Met, sandstone. } \\
\text { Met, granite gneiss; } B i=L \text {. }\end{array}$ \\
\hline $\begin{array}{l}339-R \\
340-R \\
341-R \\
342-R \\
343-A\end{array}$ & $\begin{array}{r}10 \\
\mathrm{~L} \\
\mathrm{~N} \\
\mathrm{~N} \\
\mathrm{~N}\end{array}$ & $\begin{array}{r}20 \\
\mathrm{~L} \\
\mathrm{~L} \\
\mathrm{~N} \\
\mathrm{~L}\end{array}$ & $\begin{array}{r}10 \\
N \\
10 \\
15 \\
15\end{array}$ & $\begin{array}{r}10 \\
. N \\
5 \\
5 \\
7\end{array}$ & $\begin{array}{r}300 \\
N \\
100 \\
300 \\
300\end{array}$ & $\begin{array}{r}70 \\
10 \\
300 \\
200 \\
200\end{array}$ & $\begin{array}{r}N \\
N \\
200 \\
N \\
N\end{array}$ & $\begin{array}{l}20 \\
10 \\
30 \\
10 \\
10\end{array}$ & $\begin{array}{r}\mathrm{N} \\
\mathrm{N} \\
200 \\
\mathrm{~N} \\
\mathrm{~N}\end{array}$ & $\begin{array}{r}150 \\
200 \\
100 \\
50 \\
70\end{array}$ & $\begin{array}{l}\mathrm{N} \\
\mathrm{N} \\
\mathrm{N} \\
\mathrm{N} \\
\mathrm{N}\end{array}$ & $\begin{array}{l}.08 \\
.10 \\
.24 \\
.26 \\
.16\end{array}$ & $\begin{array}{l}\mathrm{N} \\
\mathrm{N} \\
\mathrm{N} \\
\mathrm{N} \\
\mathrm{N}\end{array}$ & $\begin{array}{l}\text { Do. } \\
\text { Met, quartzite; Bi }=L \text {. } \\
(16) . \quad \\
(17) ; \\
\text { Grn; } B i=10 \mathrm{ppm} .\end{array}$ \\
\hline $\begin{array}{l}344-R \\
344 b-V \\
345-R \\
346-R \\
347-R\end{array}$ & $\begin{array}{c}\mathrm{N} \\
\mathrm{N} \\
\mathrm{N} \\
\mathrm{N} \\
10\end{array}$ & $\begin{array}{r}\mathrm{L} \\
10 \\
7 \\
15 \\
\mathrm{~L}\end{array}$ & $\begin{array}{c}30 \\
L \\
10 \\
10 \\
15\end{array}$ & $\begin{array}{r}L \\
5 \\
7 \\
15 \\
5\end{array}$ & $\begin{array}{l}300 \\
100 \\
300 \\
500 \\
200\end{array}$ & $\begin{array}{r}50 \\
200 \\
100 \\
200 \\
30\end{array}$ & $\begin{array}{l}\mathrm{N} \\
\mathrm{N} \\
\mathrm{N} \\
\mathrm{N} \\
\mathrm{N}\end{array}$ & $\begin{array}{r}10 \\
20 \\
10 \\
15 \\
L\end{array}$ & $\begin{array}{r}N \\
200 \\
N \\
N \\
N\end{array}$ & $\begin{array}{r}N \\
20 \\
150 \\
100 \\
100\end{array}$ & $\begin{array}{l}\mathrm{N} \\
\mathrm{N} \\
\mathrm{N} \\
\mathrm{N} \\
\mathrm{N}\end{array}$ & $\begin{array}{l}.24 \\
.11 \\
.30 \\
.14 \\
.07\end{array}$ & $\begin{array}{l}\mathrm{N} \\
\mathrm{N} \\
\mathrm{N} \\
\mathrm{N} \\
\mathrm{N}\end{array}$ & $\begin{array}{l}\text { Grn. } \\
\text { Met, pyritized tactite. } \\
\text { Met, grn gneiss; } B i=L \text {. } \\
\text { Do. } \\
\text { Met, gneissic grn; Bi }=L \text {. }\end{array}$ \\
\hline $\begin{array}{l}348-R \\
349-R \\
350-R \\
351-R \\
352-R\end{array}$ & $\begin{array}{l}L \\
L \\
N \\
N \\
N\end{array}$ & $\begin{array}{r}10 \\
7 \\
N \\
L \\
L\end{array}$ & $\begin{array}{l}15 \\
15 \\
10 \\
15 \\
15\end{array}$ & $\begin{array}{r}15 \\
10 \\
L \\
5 \\
L\end{array}$ & $\begin{array}{l}300 \\
300 \\
200 \\
300 \\
200\end{array}$ & $\begin{array}{r}150 \\
200 \\
10 \\
50 \\
15\end{array}$ & $\begin{array}{l}\mathrm{N} \\
\mathrm{N} \\
\mathrm{N} \\
\mathrm{N} \\
\mathrm{N}\end{array}$ & $\begin{array}{r}20 \\
15 \\
L . \\
L \\
L\end{array}$ & $\begin{array}{l}N \\
N \\
N \\
N \\
N\end{array}$ & $\begin{array}{r}70 \\
150 \\
20 \\
10 \\
50\end{array}$ & $\underset{N}{N}{ }_{N}^{N}$ & $\begin{array}{l}.12 \\
.20 \\
.16 \\
.14 \\
.16\end{array}$ & $\begin{array}{l}\mathrm{N} \\
\mathrm{N} \\
\mathrm{N} \\
\mathrm{N} \\
\mathrm{N}\end{array}$ & $\begin{array}{l}\text { Dio, xenolith, biotite dio. } \\
\text { Dio dike. } \\
\text { Grn. } \\
\text { Grn. } \\
\text { Grn, aplite dike. }\end{array}$ \\
\hline $\begin{array}{l}353-R \\
354-R \\
355-R \\
356-R \\
357-R\end{array}$ & $\begin{array}{l}10 \\
10 \\
L \\
N \\
N\end{array}$ & $\begin{array}{l}\mathrm{L} \\
\mathrm{L} \\
5 \\
\mathrm{~L} \\
\mathrm{~L}\end{array}$ & $\begin{array}{l}10 \\
20 \\
15 \\
10 \\
10\end{array}$ & $\begin{array}{l}7 \\
L \\
7 \\
5 \\
5\end{array}$ & $\begin{array}{l}300 \\
200 \\
300 \\
300 \\
300\end{array}$ & $\begin{array}{l}50 \\
15 \\
70 \\
70 \\
70\end{array}$ & $\begin{array}{l}\mathrm{N} \\
\mathrm{N} \\
\mathrm{N} \\
\mathrm{N} \\
\mathrm{N}\end{array}$ & $\begin{array}{r}15 \\
10 \\
10 \\
L \\
L\end{array}$ & $\begin{array}{l}N \\
N \\
N \\
N \\
N\end{array}$ & $\begin{array}{l}70 \\
70 \\
50 \\
50 \\
70\end{array}$ & $\begin{array}{l}\mathrm{N} \\
\mathrm{N} \\
\mathrm{N} \\
\mathrm{N} \\
\mathrm{N}\end{array}$ & $\begin{array}{l}.15 \\
.24 \\
.22 \\
.10 \\
.16\end{array}$ & $\begin{array}{l}\mathrm{N} \\
\mathrm{N} \\
\mathrm{N} \\
\mathrm{N} \\
\mathrm{N}\end{array}$ & $\begin{array}{l}\text { Grn. } \\
\text { Grn, fine grained. } \\
\text { Grn. } \\
\begin{array}{ll}\text { Do. } \\
\text { Do. }\end{array}\end{array}$ \\
\hline $\begin{array}{l}358-R \\
359-R \\
360-R \\
361-R \\
362-R\end{array}$ & $\begin{array}{c}10 \\
L \\
L \\
L \\
N\end{array}$ & $\begin{array}{l}L \\
30 \\
L \\
L \\
L\end{array}$ & $\begin{array}{r}10 \\
L \\
10 \\
10 \\
L\end{array}$ & $\begin{array}{r}5 \\
10 \\
7 \\
5 \\
5\end{array}$ & $\begin{array}{l}200 \\
300 \\
200 \\
200 \\
300\end{array}$ & $\begin{array}{r}30 \\
100 \\
30 \\
30 \\
30\end{array}$ & $\begin{array}{l}\mathrm{N} \\
\mathrm{N} \\
\mathrm{N} \\
\mathrm{N} \\
\mathrm{N}\end{array}$ & $\begin{array}{l}15 \\
10 \\
10 \\
10 \\
10\end{array}$ & $\begin{array}{l}\mathrm{N} \\
\mathrm{N} \\
\mathrm{N} \\
\mathrm{N} \\
\mathrm{N}\end{array}$ & $\begin{array}{r}150 \\
10 \\
150 \\
70 \\
150\end{array}$ & $\begin{array}{l}\mathrm{N} \\
\mathrm{N} \\
\mathrm{I} \\
\mathrm{N} \\
\mathrm{N}\end{array}$ & $\begin{array}{l}.15 \\
.14 \\
.11 \\
.18 \\
.18\end{array}$ & $\begin{array}{l}\mathrm{N} \\
\mathrm{N} \\
\mathrm{N} \\
\mathrm{N} \\
\mathrm{N}\end{array}$ & $\begin{array}{l}\text { Grn, porphyritic. } \\
\text { Dio dike. } \\
\text { Grn, porphyritic. } \\
\text { Grn. } \\
\text { Grn, porphyritic. }\end{array}$ \\
\hline $\begin{array}{l}363-R \\
364-R \\
365-R \\
366-R \\
367-R\end{array}$ & $\begin{array}{r}10 \\
10 \\
10 \\
L \\
L\end{array}$ & $\begin{array}{l}L \\
L \\
L \\
L \\
L\end{array}$ & $\begin{array}{l}L \\
10 \\
10 \\
10 \\
10\end{array}$ & $\begin{array}{l}7 \\
5 \\
L \\
N \\
5\end{array}$ & $\begin{array}{l}150 \\
300 \\
300 \\
300 \\
300\end{array}$ & $\begin{array}{l}15 \\
70 \\
50 \\
30 \\
50\end{array}$ & $\begin{array}{l}\mathrm{N} \\
\mathrm{N} \\
\mathrm{N} \\
\mathrm{N} \\
\mathrm{N}\end{array}$ & $\begin{array}{l}15 \\
L \\
L \\
L \\
L\end{array}$ & $\begin{array}{l}N \\
N \\
N \\
N \\
N\end{array}$ & $\begin{array}{r}200 \\
30 \\
100 \\
15 \\
15\end{array}$ & $\begin{array}{l}\mathrm{N} \\
\mathrm{N} \\
\mathrm{N} \\
\mathrm{N} \\
\mathrm{N}\end{array}$ & $\begin{array}{l}.16 \\
.14 \\
.08 \\
.07 \\
.09\end{array}$ & $\begin{array}{l}N \\
N \\
N \\
N \\
N\end{array}$ & $\begin{array}{c}\text { Met, grn gneiss. } \\
\text { Grn, porphyritic. } \\
\text { Do. } \\
\begin{array}{cc}\text { Drn. } & \text { Do. }\end{array}\end{array}$ \\
\hline $\begin{array}{l}368-R \\
369-R \\
370-R \\
371-R \\
372-R\end{array}$ & $\begin{array}{l}L \\
\text { L } \\
N \\
N\end{array}$ & $\begin{array}{r}L \\
L \\
70 \\
300 \\
150\end{array}$ & $\begin{array}{r}10 \\
10 \\
N \\
N \\
N\end{array}$ & $\begin{array}{r}5 \\
5 \\
15 \\
70 \\
30\end{array}$ & $\begin{array}{l}200 \\
300 \\
700 \\
500 \\
700\end{array}$ & $\begin{array}{r}30 \\
50 \\
150 \\
300 \\
300\end{array}$ & $\begin{array}{l}\mathrm{N} \\
\mathrm{N} \\
\mathrm{N} \\
\mathrm{N} \\
\mathrm{N}\end{array}$ & $\begin{array}{r}10 \\
1 \\
15 \\
70 \\
30\end{array}$ & $\begin{array}{l}\mathrm{N} \\
\mathrm{N} \\
\mathrm{N} \\
\mathrm{N} \\
\mathrm{N}\end{array}$ & $\begin{array}{l}20 \\
70 \\
30 \\
70 \\
30\end{array}$ & $\begin{array}{l}N \\
N \\
5 \\
L \\
L\end{array}$ & $\begin{array}{l}.12 \\
.13 \\
.15 \\
.06 \\
.08\end{array}$ & $\begin{array}{l}\mathrm{N} \\
\mathrm{N} \\
\mathrm{N} \\
\mathrm{N} \\
\mathrm{N}\end{array}$ & $\begin{array}{l}\text { Do. } \\
\text { Do. } \\
\text { Do. } \\
\text { Do. }\end{array}$ \\
\hline $\begin{array}{l}373-R \\
374-R\end{array}$ & $\begin{array}{l}\mathrm{N} \\
\mathrm{N}\end{array}$ & $\begin{array}{l}700 \\
300\end{array}$ & $\begin{array}{l}\mathbf{N} \\
\mathbf{N}\end{array}$ & $\begin{array}{l}15 \\
15\end{array}$ & $\begin{array}{r}30 \\
700\end{array}$ & $\begin{array}{l}70 \\
30\end{array}$ & $\begin{array}{l}\mathrm{N} \\
\mathrm{N}\end{array}$ & $\begin{array}{l}\mathbf{N} \\
\mathbf{N}\end{array}$ & $\begin{array}{l}\mathrm{N} \\
\mathrm{N}\end{array}$ & $\begin{array}{r}10 \\
\mathrm{~N}\end{array}$ & $\begin{array}{l}\mathrm{N} \\
\mathrm{L}\end{array}$ & .08 & $\begin{array}{l}\mathrm{N} \\
\mathrm{N}\end{array}$ & $\begin{array}{l}\text { Do. } \\
\text { Do. }\end{array}$ \\
\hline
\end{tabular}

${ }^{10} \mathrm{Grn}$, Met, Stream sand, Cherry Creek.

1"Quartz, Wood Vein, Jamestown, Calif., "Mother Lode" vein.

12 Met, scheelite; Cherry Creek mine, pit above adit; $\mathrm{Mn}, \mathrm{G}=5,000 ; \mathrm{W}, \mathrm{G}=10,000 \mathrm{ppm}$.

13 Met, tactite lenses; Sn, $10 ; \mathrm{Mn}, G=5,000 \mathrm{ppm}$.

14 Met, channel sample across tactite layer; K, 1.5; Ga, $0.001 ; \mathrm{Yb}, 0$.

15 Carbonized, pyritized wood; As, 700 ppm.

$16 \mathrm{Met}$, tactite (garnet, epidote, scheelite); $5 n=20 \mathrm{ppm}$.

$17_{\mathrm{Met}}$, epidote tactite; B.i $=10, \mathrm{Sn}=10 \mathrm{ppm}$.

185 gram sample.

${ }^{19}$ Roof pendant intrusive mafic rock; $<0.010 \mathrm{Pt},<0.004 \mathrm{Pd},<0.005 \mathrm{Rh}$. 


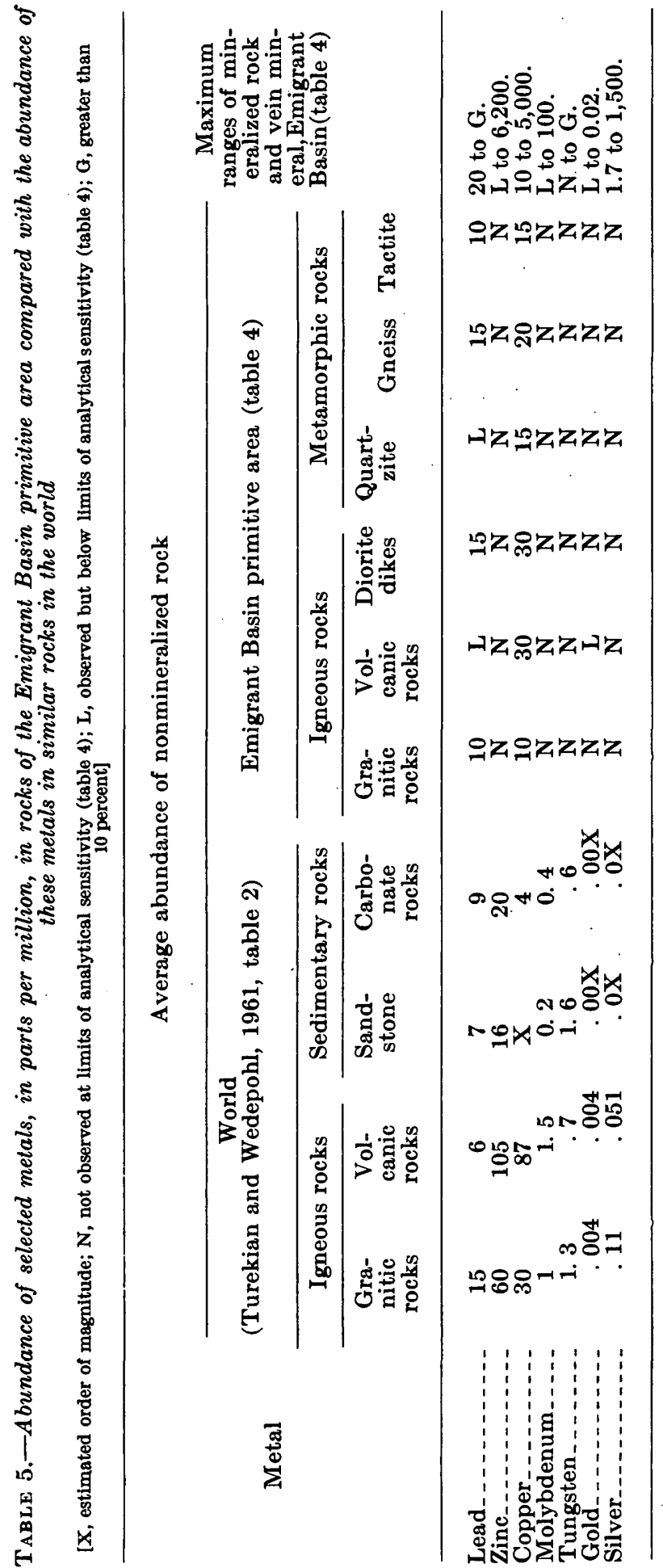


GRANITIO ROCKS OF THE BIERRA NEVADA BATHOLITH

Geochemical data from samples taken in the Emigrant Basin area indicate normal amounts of base and precious metals and other elements in the unaltered granitic rocks and in the related, but younger and less abundant, diorite, aplite, and pegmatite dike rocks. The data are consistent with those of granitic rocks elsewhere (table 5), although the analytical values are, in a general way, slightly higher than those of the Sierran granites in the Desolation Valley primitive area, California, a few miles to the north (Dodge and Fillo, 1967).

Similarly, the sediments of streams that drain the granitic terranes of the area do not contain unusual concentrations of metals. A possible exception, however, is found in the anomalous occurrence of molybdenum and tungsten in stream-sediment samples from the granite and unaltered volcanic terrane drained by Summit Creek near Relief Reservoir. Geologically, this area lies along a prelava stream channel (pl. 1, section $B-B^{\prime}$ ) that originally may have crossed the Bigelow Peak roof pendant. We infer that the slightly anomalous concentration of these elements is due to deposition of molybdenum- and tungsten-bearing minerals in a prelava stream channel. These minerals are now being reworked into the present stream sediments.

\section{VOLCANIC ROCKS}

Geochemical data from unaltered volcanic rocks indicate no anomalous metal concentrations in the Emigrant Basin area. The averages here seem to be comparable with worldwide averages (table 5). Although it is well known that uranium occurs locally in ancient stream channels buried by the volcanic rocks, as at the Juniper mine a few miles north of the primitive area, no concentrations of uranium were noted either in samples of volcanic rock or in samples of stream sediments derived from the volcanic terrane. As noted earlier, minor quantities of tungsten and molybdenum are present in sediments filling a prelava stream channel near Relief Reservoir; however, samples from the base of the volcanic rocks in other areas, as for example near Cooper Meadow, do not show comparable metal values.

\section{UNCONSOLIDATED DEPOBITS}

Except in close proximity to known mineral deposits, the sand, gravel, alluvium, and talus deposits of Holocene age do not contain unusual concentrations of ore metals or minerals. Of some interest, however, are the sands deposited in Horse Meadow in the upper part of the East Fork of Cherry Creek adjacent to the Bigelow Peak 
pendant. Locally these sands may contain lean placer accumulations of scheelite (samples $256-\mathrm{C}$ and 264-C). Similarly, the stream sediments below the old mine on Silver Mine Creek (samples 147-S to $149-\mathrm{S})$ contain anomalous metal values, attesting to the validity of geochemical prospecting techniques.

It is of further interest that gold is ubiquitous and occurs in almost all samples, but in amounts close to the limit of detection by the analytic techniques used in the geochemical study. The highest value, $0.08 \mathrm{ppm}$ gold (sample 118-S), is equal to only about 8 cents per ton.

\section{GEOCHEMISTRY OF HYDROTHERMATLY ALTERED AREAS}

During the volcanic episode and its dying phases, hydrothermal solutions moved through porous zones in the granitic and volcanic rocks pyritizing and argillizing the rocks and locally depositing small ore bodies, such as those on Silver Mine Creek and at the Juniper mine. (See p. G49-G51.) Similar areas of hydrothermal alteration were noted here and there within the primitive area, and because of their possible association with concealed ore bodies, they were carefully analyzed for traces of precious and base metals.

\section{HYDROTHERMALIY ALTERED ZONES IN GRANITIC ROCKS}

The most prominent altered granitic rocks in the primitive area crop out near the head of Cherry Lake (pl. 1). In this area two northeasterly trending zones of weathered pyritized granitic rock are localized along a group of sheeted joints. The larger of the two zones is about 600 feet wide and 5,800 feet long. At the outcrop, the rock near the center of the zone consists of strong closely spaced shears that are bleached and stained with greenish-yellow jarosite and red and brown iron oxides. Outward from the softer center channel, the rock is harder, less bleached, and less iron stained and gradually merges with the unaltered country rock. Because of the advanced state of oxidation, residual pyrite is sparse at the surface but doubtless increases with depth. No unusual magnetic properties of the zone were detected in the geophysical data.

Analyses of 10 samples taken at 50 -foot intervals across the central part of the altered area are given in table 4. From north to south, the samples are $316 \mathrm{i}-\mathrm{A}, 316 \mathrm{~h}-\mathrm{A}, 316 \mathrm{~b}-\mathrm{A}, 316 \mathrm{a}-\mathrm{A}, 316 \mathrm{j}-\mathrm{V}$ (center part), 316d-A, 316e-A, 316f-A, and $316 \mathrm{~g}-\mathrm{A}$. Sample $316 \mathrm{~g}-\mathrm{A}$ was adjacent to the southern contact with the unaltered granite; sample $316 \mathrm{i}-\mathrm{A}$ was within about 150 feet of the concealed northern contact. The trace elements are, for the most part, similar to those in unaltered granite; however, anomalous, but small amounts of copper and 
molybdenum are concentrated in the outermost altered rock, and traces of silver are present in altered rock north of the central vein. A sample $(314-\mathrm{V})$ of white quartz and sparse pyrite from joints in the granite in the main altered shear zone-the only discernible mineralized areashowed negligible amounts of metals, which may explain the lack of subsequent work done on the property.

Other, much smaller, pyritized areas in the granitic rocks, including three iron-stained joints sampled elsewhere in the primitive area (samples 13-A, 48-A, and 62-A), also show no significant concentrations of metals.

\section{HYDROTHERMALLY ALTERED ZONES IN VOLCANIC ROCKS}

Low concentrations of base metals, especially zinc, were noted in sediment samples from streams draining an area containing small altered zones in volcanic rocks near the northern boundary of the primitive area. Most of the bleached areas are adjacent to minor fractures. The zones range from a few. inches to several feet in width and are as much as a hundred feet long; however, they do not appear to persist downward for great distances. One of the zones sampled was in the Blue Canyon tributary to Deadman's Creek at the northernmost part of the district. Barite, malachite, and pyrite occur in a 2 -inch vein (sample 183-V), although not in significant quantities. Another zone, associated with a northeast-trending fracture near Leavitt Peak also contained slightly anomalous amounts of copper and silver (sample 327-A).

\section{GEOCHEMICAL SUMMARY}

The rocks of the primitive area appear to contain metals comparable in variety and quantity to similar rocks elsewhere in the world. Near known mines and prospects in the Bigelow Peak roof pendant, and downstream from suspected low-grade scheelite placer deposits, local anomalous geochemical concentrations of base metals, tungsten, and precious metals were found, as might have been anticipated. In addition, several local but, from a commercial or prospecting viewpoint, insignificant geochemical anomalies were detected in altered joint or fault zones in granitic and volcanic rocks, in ancient stream accumulations in the vicinity of the unconformity between granite and volcanic rocks, and downstream from known mineral deposits lying outside the primitive area boundary. The geochemical studies do not indicate any large unknown or concealed ore bodies in the Emigrant Basin area. 


\section{GEOPHYSICAL STUDIES}

By H. W. OLIvER, U.S. Geological Survey

\section{METHODS OF EVALUATION}

Past experience has shown that aeromagnetic surveying is a useful reconnaissance method for locating iron deposits in the Sierra Nevada (Sam Sargis, written commun., 1966). Because of their association with serpentinite, the general distribution of primary (lode) gold and silver is also outlined by magnetic patterns (Henderson and others, 1966; W. E. Yeend, oral commun., 1968; Dubois, 1963). In addition, recent tests in the northern Sierra Nevada suggest the possibility of locating detrital gold associated with black (magnetite) sands by magnetic methods (Peterson and others, 1968). Moreover, the two most magnetic minerals, magnetite and pyrrhotite, are known to be associated with scheelite-a major source of tungsten - at the Alpine tungsten mine located near Markleeville about 40 miles north of the Emigrant Basin primitive area (P. C. Bateman, oral commun., 1968) and at several localities in the Bishop tungsten district about 80 miles to the south (Bateman, 1956).

An aeromagnetic survey of the Emigrant Basin primitive area was flown along a series of east-west profiles; spaced approximately 1 mile apart, at a constant elevation of 11,500 feet above sea level. The results of the survey are presented as a total intensity magnetic map, which is overprinted on the geologic map (pl. 1). The values of the 100 gamma contour lines are the total magnetic intensities in gammas minus 50,000 gammas.

A gravity survey also was considered for the primitive area, and some density measurements were made of representative rock types. The data (table 6) indicate that there is not a sufficiently large density contrast between the various granitic rocks or between granitic rocks and the metamorphosed hornfels and quartzites of the pendants to cause meaningful anomalies. On the other hand, the high $3.38 \mathrm{~g}$ per $\mathrm{cm}^{3}$ density-value for tactite (table 6) suggests that detailed gravity studies might be useful for determining the depth to which some of the scheelite-bearing contact deposits might extend.

\section{DESCRIPTION OF MAGNETIC FIELD}

In general, after subtracting 50,000 gammas $(\gamma)$, the total magnetic intensity ranges from about 2,000 gammas in the southwest part of the primitive area to about 2,550 gammas. The high values occur within the 2,500-gamma closure just northwest of Emigrant Lake and within three smaller 2,500-gamma closures near Leavitt Peak, Relief Peak, and High Emigrant Lake (pl. 1). Thus, the total magnetic relief within the primitive area is less than 600 gammas. 
Aside from the closed contours, known as magnetic anomalies, there is a general increase in the regional magnetic values to the northnortheast from about 2,100 to about 2,300 gammas over a distance of 20 miles (pl. 1). This increase of about 10 gammas per mile is part of the earth's main magnetic field (U.S. Coast and Geodetic Survey, 1965 ) and is unrelated to local geologic units.

Magnetic anomalies in the primitive area fall into three major categories: (1) the broad high west of Emigrant Lake and the equally broad low south of Boundary Lake associated with Mesozoic granitic rocks, (2) the sharp magnetic highs located over Cenozoic volcanic rocks in the northeast part of the primitive area, and (3) a broad magnetic low and an internal magnetic high associated with the Bigelow Peak pendant in the southeast corner of the area (pl. 1). The sharpness of the anomalies is evidently related to the proximity of the airplane to the ground surface-sharpness increases as the elevation of the terrain increases to the northeast.

\section{MAGNETIC PROPERTIES OF GEOLOGIC UNITS}

In order to determine which rocks are causing the magnetic anomalies, particularly in the vicinity of the roof pendant containing the Cherry Creek mine (pl. 1), magnetic susceptibility $(\chi)$ measurements of nine of the samples collected in this area were made. Although the samples were not oriented, measurements of the remanent magnetization $(J)$ were also made for five of the samples to obtain some information on the relative importance of remanent and induced magnetization in order to interpret the magnetic anomalies. These data are given in table 6 . The field locations of the samples are shown as solid circles in figure 4. All the specimens are from the vicinity

\section{TABLE 6.-Magnetic susceptibilities, remanent magnetizations, Koenigsberger ratios, and densities of selected rock samples}

[Location of samples shown in fig. 4. Density is same as specific gravity]

\begin{tabular}{|c|c|c|c|c|c|}
\hline Rock type & $\begin{array}{l}\text { Specimen } \\
\text { No. }\end{array}$ & $\begin{array}{c}\text { Magnetic } \\
\text { susceptibility } \\
(x), \text { in } 10^{-5} \mathrm{cgs}\end{array}$ & $\begin{array}{c}\text { Remanent } \\
\text { magnetization } \\
(J), \text { in } 10^{-5} \mathrm{cgs}\end{array}$ & $\begin{array}{c}\text { Koenigsberger } \\
\text { ratio, } \\
Q=\frac{J}{.52 \chi}\end{array}$ & $\begin{array}{l}\text { Density } \\
(\rho), \text { in } \mathrm{g}^{2} \\
\text { per } \mathrm{cm}^{3}\end{array}$ \\
\hline
\end{tabular}

Granitic.rocks:

Granodiorite of $\{344-\mathrm{R} \quad 42$

Tilden Lake_... $\{369-\mathrm{R}$ Quartz-monzonite $\left\{\begin{array}{l}366-R \\ 364-\mathrm{R}\end{array}\right.$ Pendant rocks:

Tactite $341-\mathrm{R}$

Biotite schist....- $\{335-\mathrm{R}$ Hornfels_....... $\begin{aligned} & 336-\mathrm{R} \\ & 338-\mathrm{R}\end{aligned}$

Quartzite....... $340-\mathrm{R}$

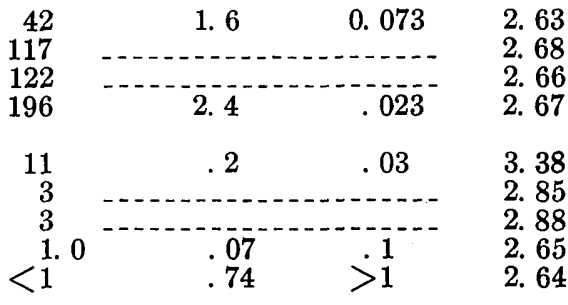


of the roof pendant except sample 364-R, which was obtained at Relief Reservoir near the north-central boundary of the primitive area.

Although they are sparse, these data indicate that the metasedimentary rocks constituting the roof pendant at Bigelow Lake are nonmagnetic $\left(x \leq 3 \times 10^{-5}\right.$ cgs (centimeter gram seconds)), except for the slightly magnetic scheelite-bearing tactite sample. In contrast, both the granodiorite of Tilden Lake and the quartz monzonite of Topaz Lake are highly magnetic $\left(\chi>10^{-3} \mathrm{cg}_{5}\right)$, although the remanent magnetization $(J)$ of these samples is low. The ratio $(Q)$ between the remanent magnetization $(J)$ and the induced magnetization $(.52 \mathrm{x})$ is less than 0.1 in both cases.

No susceptibility measurements have been made for the Cenozoic volcanic samples in this study. However, C. S. Grommé (oral commun., 1968) concluded from an earlier study that the magnetization of latite flows in the upper part of the Stanislaus Formation of Slemmons (1966) on Leavitt Peak has normal intensity for latite of about $10^{-3}$ cgs and that the direction of magnetization alternates in successive layers. Grommé and Merrill's (1965, table 5) extensive measurements on the granitic rocks, both to the north west and south of the Emigrant Basin area, are consistent with the data presented here in table 6 . Their values for the susceptibilities of 84 granitic samples are in the range 1 to $4 \times 10^{-3} \mathrm{cgs}$ and these same samples have a $Q$ ratio ranging from 0.1 to 0.3 .

\section{INTERPRETATION OF MAGNETIC DATA}

The observation that the sharpness of the magnetic anomalies increases with the elevation of the land surface suggests that the anomalies are probably caused by magnetic rocks exposed at the surface. This hypothesis has been verified quantitatively using the interpretive methods of Vacquier, Steenland, Henderson, and Zietz (1951) on a larger and more detailed edition of the magnetic map (pl. 1). Vacquier's methods were also checked on several magnetic profiles upon which the map is based, one of which is shown as $D-D^{\prime}$ on plate 1 . Vacquier utilizes the horizontal extent of the steepest magnetic gradient as a direct estimate of the depth to the top of the magnetic mass causing the anomaly. For example, the southwest flank of the Emigrant Lake anomaly has a maximum gradient of about 3,500 feet in horizontal extent in an area where the ground elevation averages about 8,000 or 3,500 feet beneath the flight elevation of 11,500 feet. Similarly, the maximum gradient on the east side of the sharp anomaly just north of Leavitt Peak is only about 700 feet, which also approximates the airplane's clearance of the 11,000-foot volcanic ridge. The conclusion that nearly all the anomalies result from rocks exposed 
at the earth's surface decreases the possibility of buried magnetic masses of possible economic significance.

If the anomalies are caused by near-surface contrasts in the magnetic properties of the rocks, then the question arises: Are the amplitudes of the anomalies consistent with the measured properties (table 6) ? In general, the answer is yes if it can be assumed that the exposed rocks extend to a depth comparable to their horizontal dimensions. For example, the main part of the Emigrant Lake anomaly, outlined by the bulge in the 2,400-gamma contour (pl. 1), has horizontal dimensions of about 5 by 6 miles or about 8 times the average height of the airplane above the ground surface (about 3,000 feet or $3 / 5$ mile). By comparing this 400-gamma anomaly with computed magnetic anomalies based on prismatic models magnetized with an inclination of $60^{\circ}$ (Vacquier and others, 1951, p. 124-128), it is evident (table 7) that a depth of at least 5 miles is required to keep the calculated susceptibility less than $2 \times 10^{-3}$ egs. (Susceptibilities have been indicated by measurements of granitic samples in table 6.) Similar calculations indicate that the susceptibilities of the exposed volcanic rocks required to produce the sharp anomalies of about 250 gammas near Leavitt Peak, Relief Peak, and High Emigrant Lake are of the order of $10^{-3} \mathrm{cgs}-\mathrm{a}$ reasonable value for these rocks. Thus, all the large magnetic features within the Emigrant Basin primitive area can be explained in terms of the magnetic properties of exposed rock types.

TABLE 7.-Dependence of the susceptibility contrast required to produce the Emigrant Lake anomaly on the assumed depth extent of the magnetic mass

\begin{tabular}{lll} 
Depth extent of magnetic mass below the ground surface, in miles & $\begin{array}{c}\text { Calculated } \\
\text { susceptibility } \\
(\chi) \text {, in 10-3 cgs } 1\end{array}$ \\
\hline & & 6.4 \\
\hline
\end{tabular}

\footnotetext{
1 Based on Vacquier's (1951, p. 18) formula where

$\Delta T_{m}$ is the total amplitude of the actual anomaly (400 gammas)

$\Delta T_{\mathrm{c}}$ is the total amplitude of the intensity anomaly computed for various models, and

$T$ is the intensity of the earth's main magnetic field in gammas $(52,100)$.
}

Section $D-D^{\prime}$ (pl. 1) shows a direct correlation between the magnetic properties and the geologic units (granite plutons and roof pendant rocks). For example, the granitic unit underlying the Wood Lake area causes a magnetic high, whereas the unit underlying the Grouse Lake area is much less magnetic. The magnetic high west of Emigrant Lake probably delineates a pluton, or group of plutons, within the Sierra Nevada batholith similar to the Sentinel Granodiorite of Yo- 
semite Valley. These plutons must extend north-northwest under the Cenozoic volcanic rocks where the magnetic ridge, marked by the 2,400gamma contour, crosses the north boundary of the primitive area near Castle Rock. A higher level survey (16,000 feet) of the Sierra Nevada between $3712^{\circ}$ and $39^{\circ} \mathrm{N}$ (Zietz and Kirby, 1968) shows that this magnetic ridge strikes north-northwest for a distance of about 35 miles and must represent a major lineament in the Sierra Nevada batholith.

The character of the magnetic field associated with the Bigelow Peak pendant is of special interest because of the scheelite deposits at the Cherry Creek and Montezuma mines (pl. 1). A magnetic profile taken through the center of the pendant (section $D-D^{\prime}$, pl. 1) shows a broad magnetic low of almost 400-gammas centered just east of Bigelow Lake. The small internal anomaly marked " $c$ " in the profile (pl. 1) is the expression of a south-plunging magnetic nose near Bigelow Lake.

The main low is expectable because of the nonmagnetic character of the metasedimentary rocks (table 6 ). The anomaly is caused in part by the alaskite of Grace Meadows (Clyde Wahrhaftig, oral commun., 1968), which lies directly under the eastern part marked " $d$ " on section $D-D^{\prime}$ (pl. 1). The anomaly may also be, in part, an offshoot of a larger magnetic low centered off the map just south of Twin Lakes and lying over the El Capitan Granite-a pluton known to be essentially nonmagnetic. The internal high is probably caused by the mafic intrusions within the pendant, although the closest outcrop of hornblendite and gabbro is 1,000 feet south of the flight line (pl. 1).

\section{ECONOMIC SIGNIFICANCE OF MAGNETIC DATA}

Despite the fact that the magnetic data obtained for the study are of a reconnaissance nature, experience has shown that a 1-mile spacing of flight lines is adequate to find major anomalies and to pick up about 70 percent of the minor features. The weakest data control in this area is in the western part where the height of the airplane above ground exceeded 3,000 feet.

Within the framework of these limitations, the following conclusions can be drawn:

1. There is no magnetic evidence for large concealed bodies of tungsten-bearing tactite along margins of roof pendants. This does not preclude the possibility that nonmagnetic tactites are present.

2. Because of the total magnetic relief of only 600 gammas, it is safe to say that there are no commercial iron (magnetite) deposits in the primitive area. 
3. There are no serpentinite bodies, at least of the universally magnetic type that serve as guides to gold and silver lodes in the western Sierra.

4. There is no indication of strongly magnetic placers. The magnetic patterns and anomalies that do occur in the primitive area can all be explained in terms of the measured magnetic properties of the rocks exposed at the surface.

\section{MINERAL RESOURCES}

The mineral deposits or mineral resources of the Emigrant Basin primitive area include (1) possible extensions of known deposits of uranium, base and precious metals, and tungsten that occur in nearby areas, (2) mines and prospects that are known to contain tungsten, (3) newly located undeveloped mineralized and altered localities, and (4) possible undiscovered or concealed ore bodies, particularly in the Bigelow Peak roof pendant but also in the volcanic or granitic rocks.

The term "mineral resource", as used in this report, upplies to mineral and rock materials that are being mined or may be mined in the foreseeable future. The term refers to metallic, nonmetallic, and industrial mineral deposits that are known to occur or may be expected to occur within the area because the geological environment is favorable. At the time this study was made (1967-68) only one mineral deposit in the primitive area was being prepared for mining; however, prospecting and development of mineral claims, chiefly for tungsten, was being actively pursued. The occurrence of scheelite, sphalerite, molybdenite, and other minerals, which are common in the roof pendant in the southeastern part of the primitive area, are geologically similar to economic deposits elsewhere in the Sierra Nevada (Krauskopf, 1953, and Bateman, 1965) and may or may not eventually prove to be of important commercial interest.

\section{NEARBY PRODUCTIVE DEPOSITS}

\section{JUNIPER MINE}

The Juniper mine, which was discovered in 1955 and is the largest uranium mine in California, is situated about 3 miles northwest of the northern boundary of the Emigrant Basin primitive area in NE $1 / 4$ sec. 8 , T. 5 N., R. 20 E. The mine and nearby prospects are located on the ridge separating the drainage basin of Long Valley Creek from that of the Middle Fork of the Stanislaus River. The main workings, including a large open pit; are at an elevation of about 8,500 feet and are accessible during the summer season by a a mountain road extending from State Highway 108 at Niagara 
Creek. Several other uranium deposits have been prospected in the Niagara Creek drainage several miles to the west of the Juniper deposit.

According to E. H. Boudreau (J. B. Koenig, oral commun., 1967), the Juniper ore body is localized in a prevolcanic stream channel near the base of flat-lying sedimentary rocks, which consist of unconsolidated to well-cemented sandstone, conglomerate, tuff, and claystone. The sedimentary rocks overlie hematite-stained granitic rocks and in turn are overlain by andesitic flow rocks and breccias. "Upstream" along the old stream channel, the granitic and volcanic rocks are cut by dikes and plugs of hornblende andesite prophyry, which may be related to the deposition of the ore bodies.

The ores of the Juniper deposit are intimately associated with fossil plant remains, carbonaceous trash, and pyritized wood. The ore minerals consist mainly of autunite and torbernite (green and yellow hydrous calcium-copper-uranium phosphate minerals) in the oxidized near-surface parts of the ore zone, and a fine-grained black mineral, probably uraninite, in the unoxidized zone below the water table. Spectrographic analyses of the ores indicate the presence of vanadium, cobalt, nickel, molybdenum, and lead, but not in recoverable quantities. In addition, the strongly pyritized clastic rocks in the ancient stream channel adjacent to the ore bodies contain trace concentrations of uranium, arsenic, and mercury and lower, but still anomalous, amounts of molybdenum, boron, and zinc.

The ore bodies at the Juniper mine occur as discontinuous layers and lenses in a sinuous linear zone extending about 4,500 feet along the ancient stream channel. According to E. H. Boudreau (J. B. Koenig, oral commun., 1967) about 6,000 tons of uranium ore averaging 0.35 percent of $\mathrm{U}_{3} \mathrm{O}_{8}$ were shipped in 1963 and 1964 to Salt Lake City, Utah. In addition, an unstated but smaller production for 1965 and 1966 is recorded by the U.S. Bureau of Mines (Davis, 1967a, p. $156 ; 1967 \mathrm{~b}$, p. 156). Although no reserve estimates are available, additional undiscovered deposits may be present in unexplored parts of other buried stream channels in the area. Some of these channels were being explored in June 1968 by the Utah Construction and Mining Co.

Uranium also occurs in podlike masses at the Autunite group of mining claims of the Carrigan Mining Co., in secs. 4, 5, 8, and 9, T. 5 N., R. 19 E., Tuolumne County, Calif., in the central part of the Niagara Creek drainage basin. These deposits, according to $\mathrm{H}$. K. Stager (written commun., 1967) are similar to those at the Juniper mine, but no production has been recorded, although some samples contained as much as 0.13 percent $\mathrm{U}_{3} \mathrm{O}_{8}$. 
Silver Mine Creek, which flows into the west-central part of Relief Reservoir, is named from a prospect located about half a mile beyond the northwest boundary of the primitive area and close to the sharp bend in the creek near the center of sec. 14, T. 5 N., R. 20 E. In 1967 the property was reportedly owned by Miller Sardella, but it was not being worked when examined in September and October of that year.

The country rock near the prospect is the porphyritic quartz monzonite of Topaz Lake that is cut by several andesitic dikes 10 to 30 feet wide and about a hundred feet long. The ore minerals include enargite, galena, sphalerite, and pyrite in branching and coalescing white quartz veins $1 / 2$ to 6 inches thick. The veins follow a pyritized sheeted zone a few feet thick striking approximately N. $20^{\circ}$ E. and dipping west at $45^{\circ}$ or less. In addition to quartz, the gangue minerals include chlorite, ankerite, and earthy hematite. The pyritized zone, which is now weathered, bleached, and iron stained, crops out for a quarter of a mile or more east of the prospect, but the quartz veins are apparently limited to the area of the prospect itself. Similar pyritized zones form a selvedge along the contacts of other dikes in the Silver Mine Creek area, but no other mineral deposits were observed.

The main vein zone was originally opened by a pole-timbered inclined shaft that was sunk in the bed of Silver Mine Creek after the stream had been diverted into an artificial channel by means of a small earth-fill dam. A later flood apparently breached the dam and filled the shallow shaft with alluvium and debris.

Ore production from the prospect appears to have been negligible, and the potential for commercial ore is believed to be small.

\section{PROSPECTS NEAR DOROTHY LAKE}

The Dorothy No. 1 and Dorothy tungsten prospects are in the northeast corner of Yosemite National Park in the NE1/4 sec. 30 and SE $1 / 4$ sec. 19, T. 4 N., R. 22 E., and in sec. 25, T. 4 N., R. 21 E, near the southeast corner but outside of the Emigrant Basin primitive area The prospects were discovered in 1941 (Tucker and Sampson, 1941). In 1943 about 55 tons of ore averaging 70 percent $\mathrm{WO}_{3}$ was packed out by mule from Dorothy No. 1 (Lemmon and others, 1943). The ore body in the Dorothy No. 1 was 6 feet wide and 60 feet long, but the high-grade ore exposed at the surface was underlain at shallow depth by barren quartzite and mining was soon abandoned.

According to Lemmon, Bateman, Stagner, and Beatty (1943) the scheelite occurs in garnet-epidote tactite interbedded with quartzite, schist, and hornfels. It crops out at the east edge of one of the satellitic bodies of the Bigelow Peak roof pendant, close to the contact with the granitic batholith. Numerous granite sills cut the metamorphic rocks 
near the granodiorite contacts and locally form migmatites. The metamorphic rocks are deformed and faulted, the general structure apparently being a north-plunging, northwest-striking, faulted syncline. The scheelite occurs as irregular disseminations in and along fractures in the rock; these fractures probably represent small faults along which the solutions moved. The scheelite crystals range from a quarter of an inch to 2 inches in size, and the $\mathrm{WO}_{3}$ content of the rock was estimated to range from 1 to 30 percent.

The Dorothy prospect, a quarter of a mile to the west of the Dorothy No. 1, is in an area underlain chiefly by schist, hornfels, and marble. The rocks strike northwest and dip steeply to the southwest and are entirely surrounded by granodiorite. A tactite bed 6 feet thick extends along the contact between the schist and the marble. Most of this tactite bed is barren of commercial minerals, but on its western edge, close to its contact with the marble, there is a narrow streak of tungsten ore that ranges from 0.5 to 1.5 percent $\mathrm{WO}_{3}$. The streak extends about 380 feet along the strike, but its maximum width is only 6 inches.

\section{MINES AND PROSPECTS WITHIN THE PRIMITIVE AREA}

\section{MINING CLAIMS}

The records of mineral-claim locations and proofs-of-labor filed in Tuolumne County indicate that 44 claims, of which 42 remained valid in 1968, were located in the Emigrant Basin area from 1942 through 1956. All of the claims list tungsten as the metal of principal interest, and all are in T. 4 N., R. 21 E., MDBM (Mount Diablo baseline and meridian) in the southeastern part of the primitive area (fig. 11).

On September 3, 1965, the owners of one of the groups of claims (Whittle property) applied for a patent on four of their claims. The Forest Service granted a certificate of approval for one of the claims on February 3, 1967, and granted the owners an extension of time to do additional exploration on the other three pending final action on the first patent application.

In addition to general studies of the mineralized areas during the geological investigations by $\mathrm{E}$. W. Tooker and H. T. Morris, detailed on-site field inspections of the principal claims were made by Paul V. Fillo in July 1966, September 1967, and August 1968. The 1966 trip was limited to inspection and sampling of four claims of the Whittle group in response to the owner's application for mineral patent. This inspection was made in the company of William L. Johnson, of the U.S. Forest Service. The 1967 trip was undertaken to examine the Whittle property, the Montezuma mine, and the tactite zones in the vicinity of these mines. During the 1968 investigation, the tactite zones in the vicinity of the Buckskin and Bigelow groups of claims were examined. 


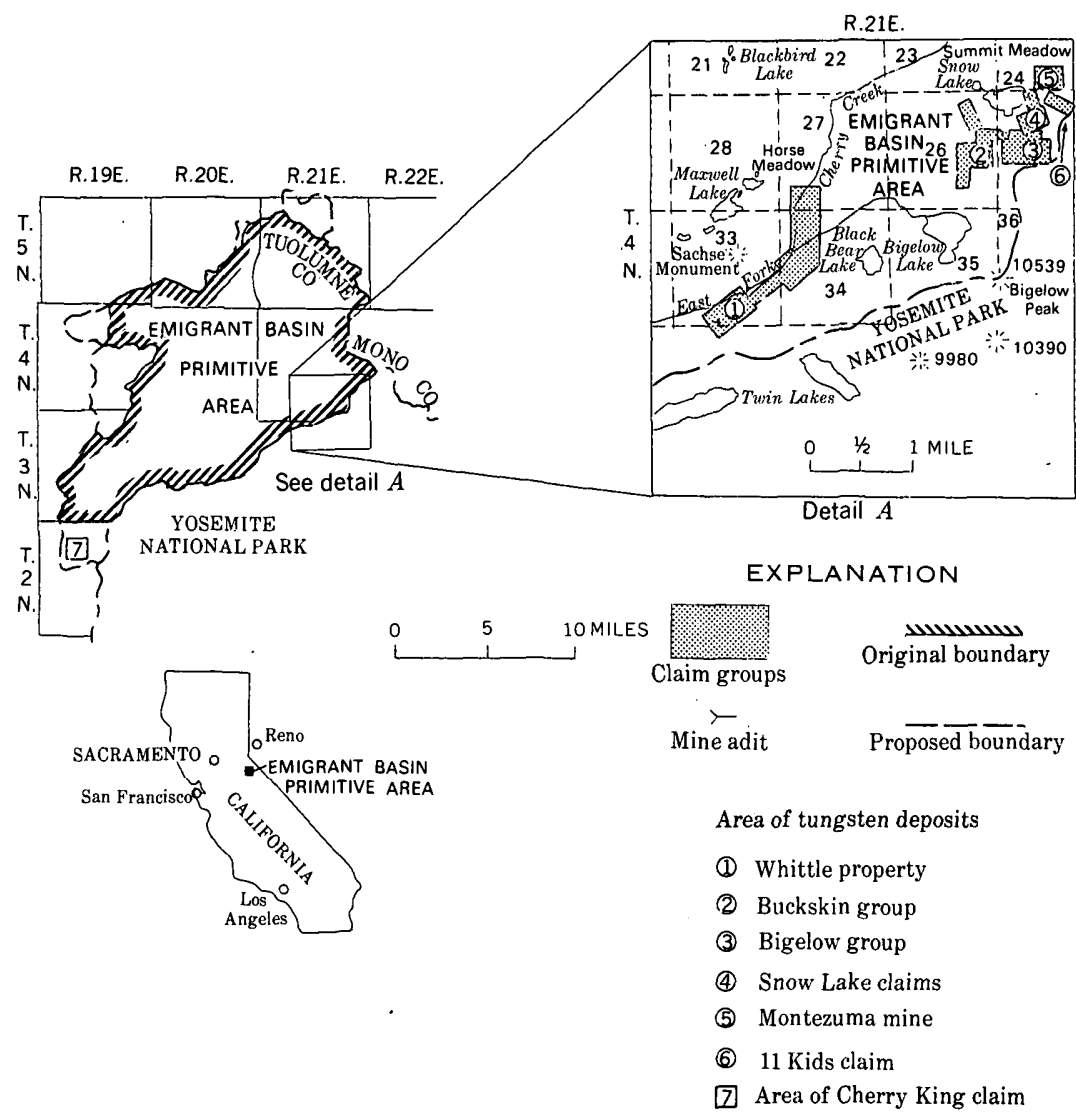

FrgURE 11.-Map of Emigrant Basin primitive area showing proposed wilderness boundary and sites of mining and prospecting activity.

The Cherry King claim, in the southern part of the study area, filed in 1955 by D. B. Woodruff and Elmer Shaw, as a "quartz claim," was sampled by E. W. Tooker and H. T. Morris in June 1968. No other examinations of this claim were made, because no unusual metal concentrations were determined by geochemical studies (p. G42) and no visible development activity has taken place since the filing.

No Federal oil and gas leases and no Federal petroleum or phosphate reserve lands are recorded within the primitive area.

\section{TUNGSTEN DEPOSITS}

All of the tungsten deposits in the primitive area are in the metamorphosed sedimentary rocks and undifferentiated small intrusive bodies of the Bigelow Peak roof pendant between the East Fork of Cherry Creek and the border of Yosemite National Park. The princi- 
pal deposits are in three general localities: (1) in the vicinity of Sachse Monument, (2) near Snow Lake, and (3) near Bigelow Lake. The most important showings of tungsten in these three areas are described below by mines or by groups of claims in descending order of potential for tungsten production.

\section{WHITTLE PROPERTY}

The Whittle property (also known as the High Sierra or Cherry Creek mines) consists of 21 lode claims and 2 placer claims located in secs. 27, 33, and 34, T. 4 N., R. 21 E. (fig. 11). The claims of the group are: Tamarack, Marlyn, Marie Whittle, Jan Cavanaugh, Francis Finley, Garnet, Ventura, Airola 1 and 2, El Venado, Michael, Tungsten Hill (claims 1 through 4), Gladstone, Mary Whittle, Bussy, Donna, Pleasant View, and Angie Whittle lode claims, and Horse Meadow 1 and 2 placer claims. A small production of tungsten from the Whittle property was reported to the Bureau of Mines in 1955, 1956, 1962, and 1967.

The claims were located by Harry W. Whittle, Donald R. Whittle, and associates in 1954 and 1955. They are contiguous and are located on both sides of Cherry Creek from Horse Meadow, the headwaters of Cherry Creek, west-southwest to the Cherry Creek mine in sec. 33, T. 4 N., R. 21 E., near Sachse Monument. In September 1967, the property was under lease to the Cherry Creek Mining Company of San Francisco and was being developed for production under the supervision of the late Mr. John Loring.

The property is reached by a restricted-access road branching from State Highway 108 at Leavitt Creek about 3.2 miles east of Sonora Pass. This narrow road has several steep grades and many switchbacks and crosses the summit at an elevation of 9,700 feet; for these reasons it can be negotiated only by vehicles with a short wheel base and fourwheel drive. The road follows the Mono-Tuolumne County boundary in a southerly direction to Emigrant Meadow and past Grizzly Peak to Summit Meadow, the headwaters of Cherry Creek. In Summit Meadow the road forks; the left fork leads to the Montezuma mine near Snow Lake, a distance of about 1 mile, and the right fork leads in a southwesterly direction along the East Fork of Cherry Creek for about 3.7 miles to the Cherry Creek mine where the road ends.

Mineral occurrences at the Whittle property are generally typical of all of the tungsten deposits in the Bigelow Peak roof pendant. Discontinuous lenses and beds of limestone have been metamorphosed to marble and to garnet-epidote tactite at or near the contacts of the roof pendant and the granitic rocks. The pendant rocks are tightly folded, and the limy beds have locally been squeezed, distended, and 
separated into podlike masses of unpredictable shape. At the Cherry Creek mine, an opencut 60 feet above the adit exposes a pod of disseminated tungsten ore in tactite that is about 20 feet wide by 20 feet long at the surface and may extend to a depth of 40 or 50 feet. Scheelite crystals as much as $11 / 2$ inches long and 1 inch across are not uncommon in the ore. The quartzite, hornfels, and marble beds associated with the tactite lens appear to dip steeply and regularly to the east, but the tactite layer was not found in the adit below the ore outcrops.

Other scheelite occurrences in the Sachse Monument area are directly related to the abundance of silicated limestone (tactite) in the roof pendant host rocks. These sparsely mineralized tactite bodies commonly crop out as narrow, steeply dipping, iron-stained pods and lenses that are interlayered with much thicker beds and lenses of massive white to buff quartzite, white marble, and black to rusty brown hornfels. Their lenselike character and their common occurrence as saddle-shaped pods at the apices of minor folds makes it virtually impossible to predict their continuity either in depth or along the strike in covered areas. At the Whittle property the extension of the ore shoots to depth has not been determined. The downward extension could be limited for one or more of the following reasons: (1) squeezing out of the limestone bed during the intense folding that preceded metamorphism, (2) a decrease in tungsten content with depth (the grade of tungsten ore shoots is commonly erratic), and (3) termination in depth of the tactite lenses at the contact of the underlying granitic mass.

The Jan Cavanaugh, Francis Finley, Garnet, and Tamarack claims on the western end of the Whittle property contain isolated tactite bodies that appear to be tapered masses that project downward into the granitic rocks. The four claims have been prospected more thoroughly than the rest of the claims in the group. The open cuts where samples were taken are shown in figure 12 .

The four claims were checked at night with an ultraviolet lamp; concentrations of the fluorescent scheelite were marked by flags and, where justified, samples were taken later during the daylight hours. The flags were placed in a manner that made it easy to map and sample the entire surface exposure. All samples were cut from zones showing scheelite, but some scheelite zones were not sampled when evidence indicated that only a small pod or thin fracture coatings were present.

\section{JaN CAvaNaUgh CLAim}

This claim consists of four open cuts and an adit 110 feet long. The adit was driven 60 feet below the discovery cut in granitic rock that contains local concentrations of quartz and epidote. Chip samples were 


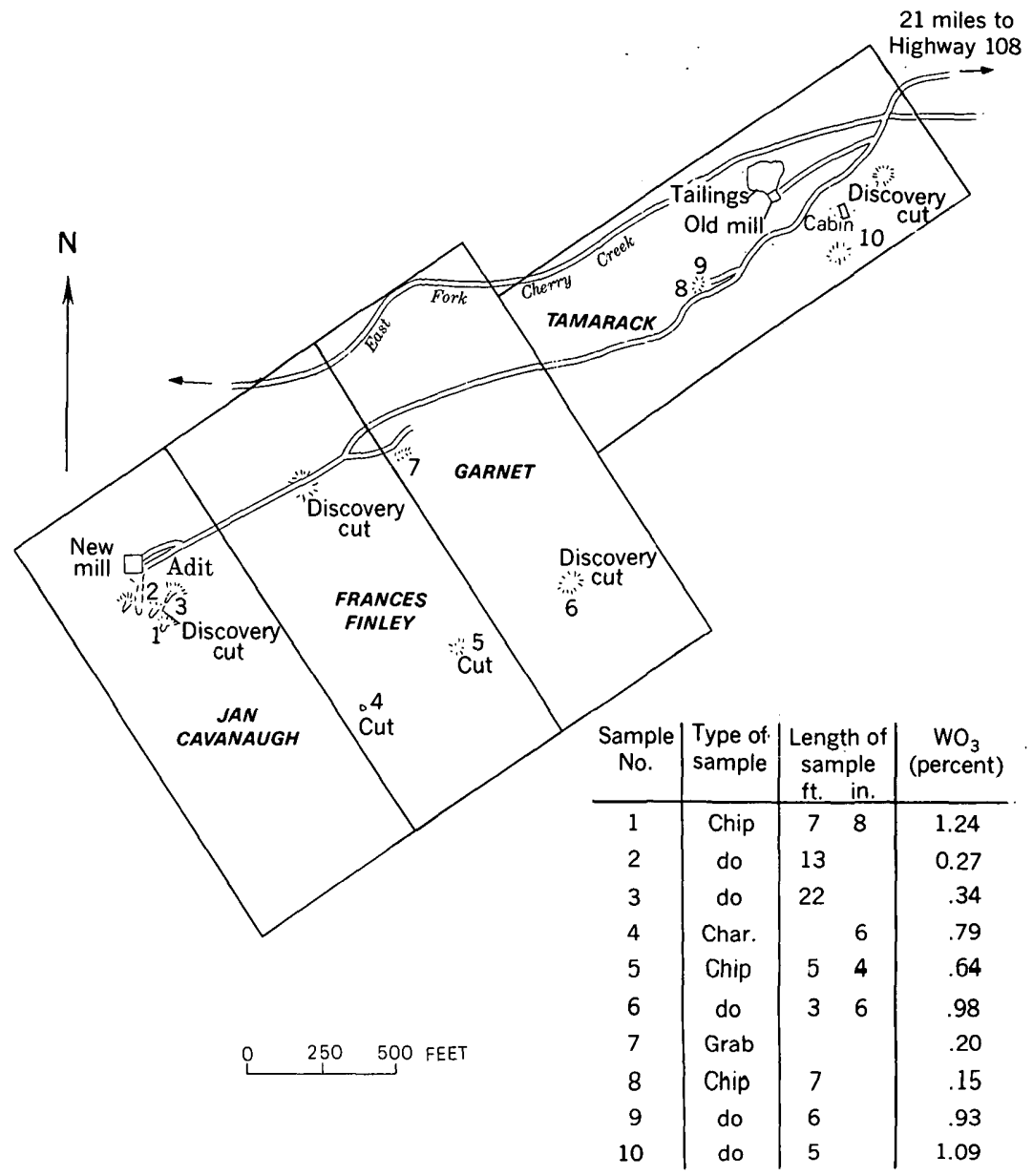

FIGURE 12.-Claims, surface development, and sample locations in the western part of the Whittle property.

taken from three open cuts in scheelite-bearing tactite zones (fig. 12). The assay results were $1.24,0.27$, and 0.34 percent $\mathrm{WO}_{3}$. A small tonnage of selected ore was produced, chiefly from the discovery cut on the claim, and was shipped to the Union Carbide Corp. plant near Bishop, Calif. The tactite zones exposed on the claim may constitute a potential for future production of tungsten ore. It is estimated from sampling and the exposures in the pits and on the surface that possibly several thousand tons of tungsten ore of a grade approximating 0.6 percent $\mathrm{WO}_{3}$ are present.

A mill with a planned capacity of 100 tons of scheelite ore per day was built on the claim in 1967 (fig. 13). The mill equipment includes 
a 12 - by 20 -inch primary jaw crusher, an 8 - by 24 -inch secondary jaw crusher, a set of 4-inch crushing rolls, screens, and two wet gravity concentrating tables. Electric power for the mill is furnished by two 30-kilowatt generators powered by diesel engines. From all appearances, only a small tonnage of ore has been milled.

Most of the ore produced from the Whittle property probably came from the Jan Cavanaugh claim; however, the exact quantity of ore produced is not known.

\section{FRANCIS FINLEY CLAIM}

Development work on this claim consists of three open cuts. During preliminary examination with an ultraviolet lamp, two scheelitebearing tactite zones were observed and sampled. A character sample containing 0.79 percent $\mathrm{WO}_{3}$ was taken from one zone. A chip sample was taken across the widest part of the second zone. This zone consists of a tactite-scheelite lens ranging from less than 1 inch to more than 5 feet in width and having a strike length of 80 feet. The sample assayed 0.64 percent $\mathrm{WO}_{3}$. About 1,000 tons of tungsten ore assaying 0.6 to 0.8 percent $\mathrm{WO}_{3}$ is estimated to be present, but no ore has been produced.

\section{GARNET CLATM}

Development work on the Garnet claim consists of two open cuts. The discovery cut exposes a scheelite-bearing tactite zone approximately 4 feet wide, 30 feet long, and at least 50 feet thick. A sample from one cut assayed 0.98 percent $\mathrm{WO}_{3}$. A grab sample from three places in a bulldozer cut averaged 0.20 percent $\mathrm{WO}_{3}$. A small tactite zone, 3 by 6 feet in surface area, in the southeast section of the claim (not shown on fig. 12) contains stringers of scheelite. Only a few hundred tons or ore are inferred, and no ore has been produced.

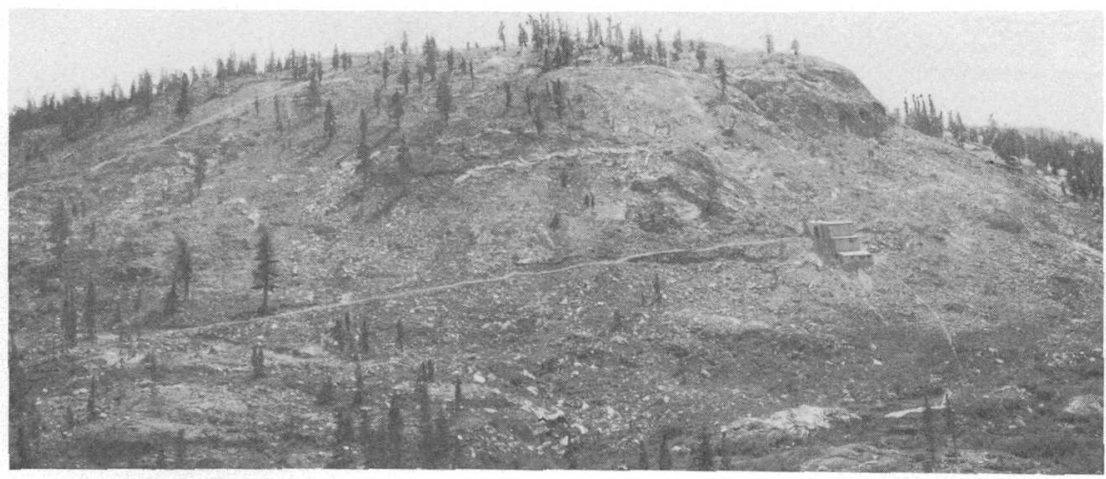

FIgURE 13.-Western part of the Whittle property. Gravity concentrating mill is to the right in the photograph. 
TAMARACK CLAIM

The Tamarack claim lies on a relatively flat topographic bench about 20 feet above Cherry Creek, which generally follows the north line of the claim. A sample from a small pod of scheelite-bearing tactite assayed 0.15 percent $\mathrm{WO}_{3}$. A sample from a northerly striking tactite zone 2 inches or less in width and 40 feet in length assayed 0.93 percent $\mathrm{WO}_{3}$. A 5-foot chip sample taken across an open pit assayed 1.09 percent $\mathrm{WO}_{3}$. The extension of these small lenses to depth is unknown. At several locations on the claim a thin veneer of scheelite also occurs on fracture faces, but such showings did not warrant sampling.

On the basis of the small size and discontinuity of the tactite zone and the sampling, the Tamarack claim has no apparent potential for development. Other small tactite zones are exposed near the Tamarack claim and are estimated to have a potential of several hundred tons of ore. Additional exploratory work would be required to evaluate their importance.

\section{MONTEZUMA MINE}

The Montezuma property consists of the Montezuma No. 1 and Montezuma No. 2 lode claims; both were located on August 24, 1942, by L. W. Osborn, W. H. Heitman, and associates. The claims are in sec. 24, T. 4 N., R 21 E., on the east side of Snow Lake (fig. 11). The claims are reached from the road to the Whittle property by a 1-milelong spur road that turns east at Summit Meadow.

The workings of the Montezuma mine, which were developed in the early 1950 's by the locators, total approximately 215 linear feet of adit and drift and a shallow winze, all terminating in granitic rocks near the contacts with the roof-pendant rocks. In addition, several open cuts were excavated early in the history of the claims, apparently in an attempt to delineate mineralized zones. Twelve tons of selected ore, averaging 1.6 to 2.7 percent $\mathrm{WO}_{3}$ are reported to have been packed out by mules in 1951. Small quantities of tungsten concentrate were also produced and shipped from the workings and dumps on the claims in 1953 and 1954. Cut samples from the underground workings assayed from 0.01 percent to 0.05 percent $\mathrm{WO}_{3}$. Three of the samples contained 0.01 percent Mo (molybdenum).

The Montezuma mine adit exposes the contact of roof-pendant rocks with granodiorite. Epidote-garnet tactite, containing fine- to coarsegrained scheelite and minor amounts of copper- and molybdenumbearing minerals, is exposed here and there in this contact zone (fig. 14). Some of the scheelite is concentrated in layers parallel to the metamorphic banding in the rocks, but most of the ore mined consisted of scheelite disseminated throughout the host rock. The largest body of tactite exposed in the adit is about 70 feet long. Scheelite-bearing 

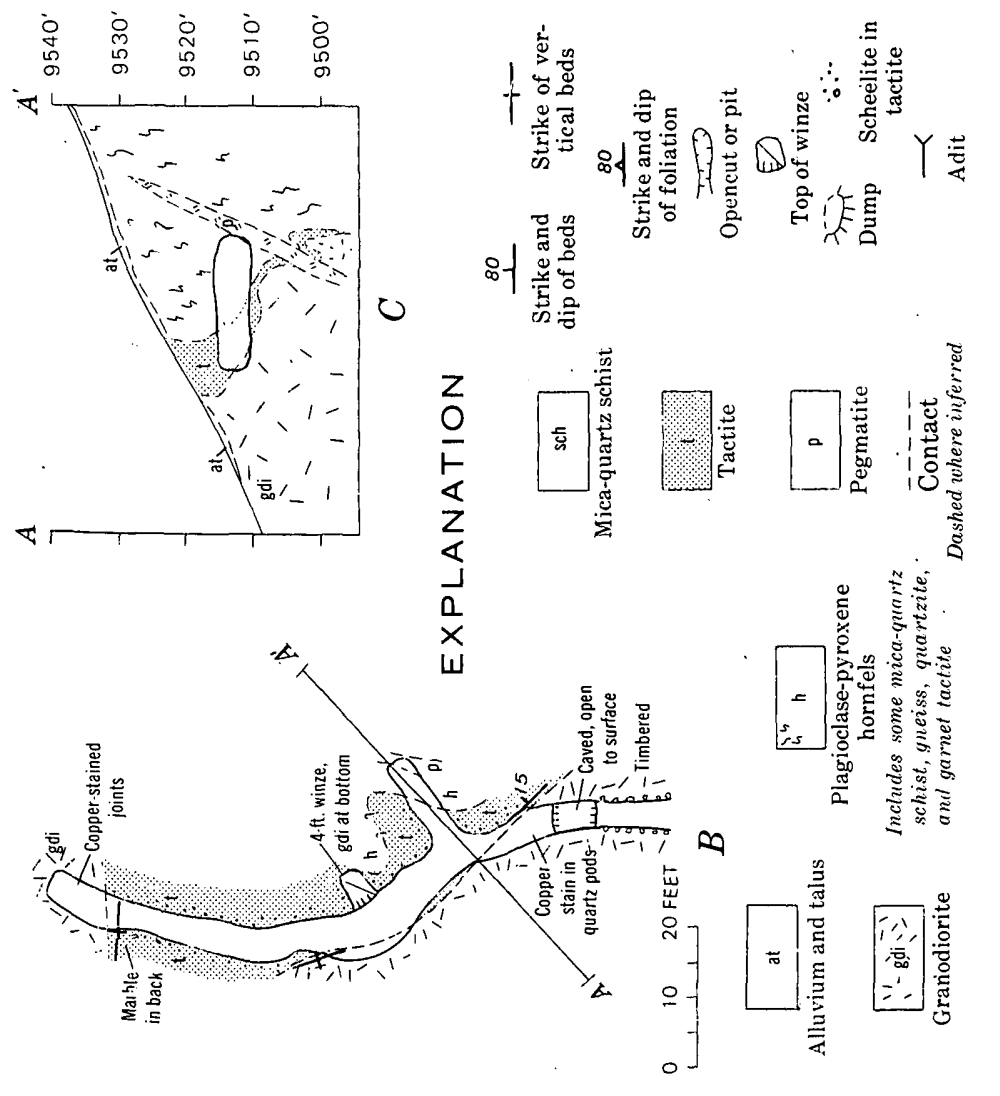

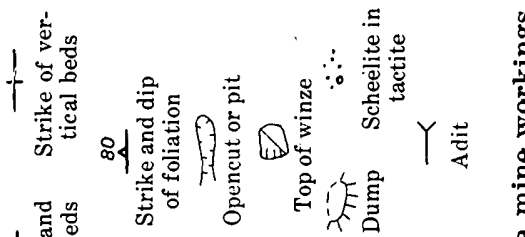

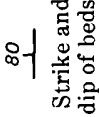

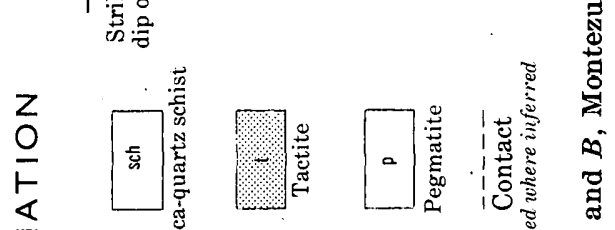

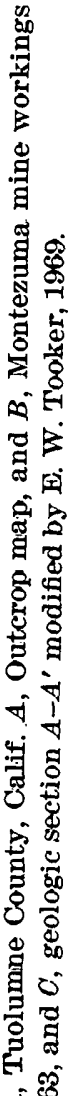

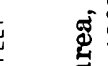

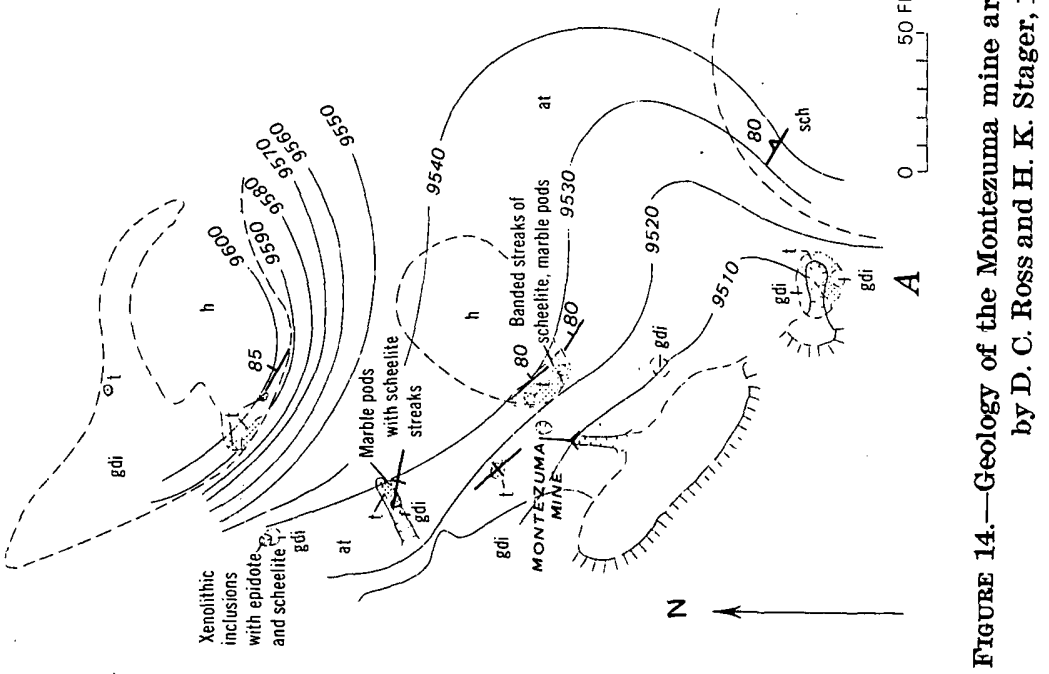


tactite is also exposed in the small surface pits and open cuts, and several pods of tactite 1 to 5 feet long are exposed in the granodiorite and in hornfels 100 to 200 feet north of the main workings.

The future commercial success of the Montezuma mine is in large part dependent on the depth below the surface to which the roofpendant rocks and, specifically, the tactite lenses persist. The bottom of the winze that is located about 50 feet inside the portal exposes granodiorite. This exposure leads H. K. Stager and D. C. Ross (written commun., 1966) to believe that the pendant is a shallow body; diamond drilling in the floor of the adit also intersected granodiorite at a shallow depth. The occurrence of other xenoliths of metamorphic rocks at depth, however, is possible and even likely.

\section{BIGELOW GROUP}

The six claims of the Bigelow group are the Bigelow Peak, Bigelow, and Bigelow Nos. 1 through 4. The first two claims were located on July 23, 1954, and the last four claims on September 8, 1954, by F. W. Hanson. The claims are contiguous and are in sec. 25, T. 4 N., R. 21 E. They can be reached by a road that branches northeastward from the Montezuma mine road approximately half a mile from the Cherry Creek mine road. The distance from the Montezuma mine road junction to the road terminus at the Bigelow Peak claim is approximately 2.5 miles.

The roof pendant in the area of Bigelow Peak and Bigelow Lake consists of interbedded quartz-mica schist, quartzite, and marbleall are highly granulated and folded and are intruded by numerous dikes and sills of aplite and pegmatite (fig. 15). Small lenses and thin beds of limestone have been metamorphosed into tactite and are the host rocks for scattered sparse grains of scheelite. These rocks, in general, strike northwest and dip steeply southwest and are cut by several faults of unknown, but probably small, displacement. The scheelite-bearing tactite forms irregular small layers or lenses 2 to 30 feet wide and 10 to 70 feet long and concordant with the steeply dipping host rocks. Some of the mineralized layers extend discontinuously for more than 1,000 feet; their depths are unknown. No production has been recorded.

Exploration work on the group has been concentrated on the Bigelow Peak claim and consists of four shallow pits which penetrate discontinuous tactite lenses. The pit faces were sampled; the location, length, and assay results of the four samples taken are shown in figure 16. The assay results range from 0.16 to 0.91 percent $\mathrm{WO}_{3}$.

The sample results, the observed continuity of some of the scheelite zones, and the estimated depth to which the mineralization is believed to extend indicate a small minable reserve-perhaps a few hundred tons-on the Bigelow Peak claim. 


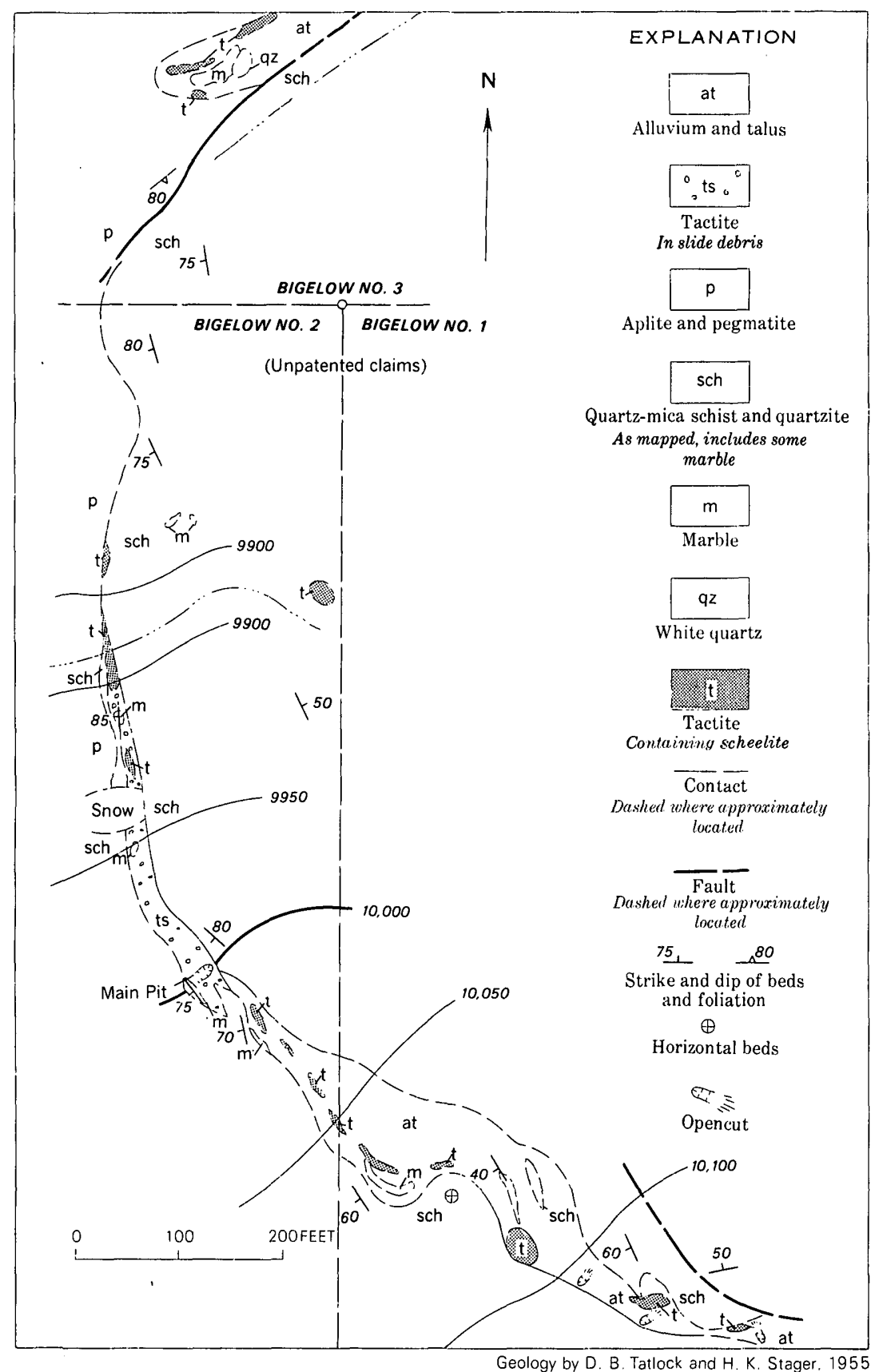

Figure 15.-Geologic map of part of the Bigelow group of claims, Tuolumne County, Calif. 


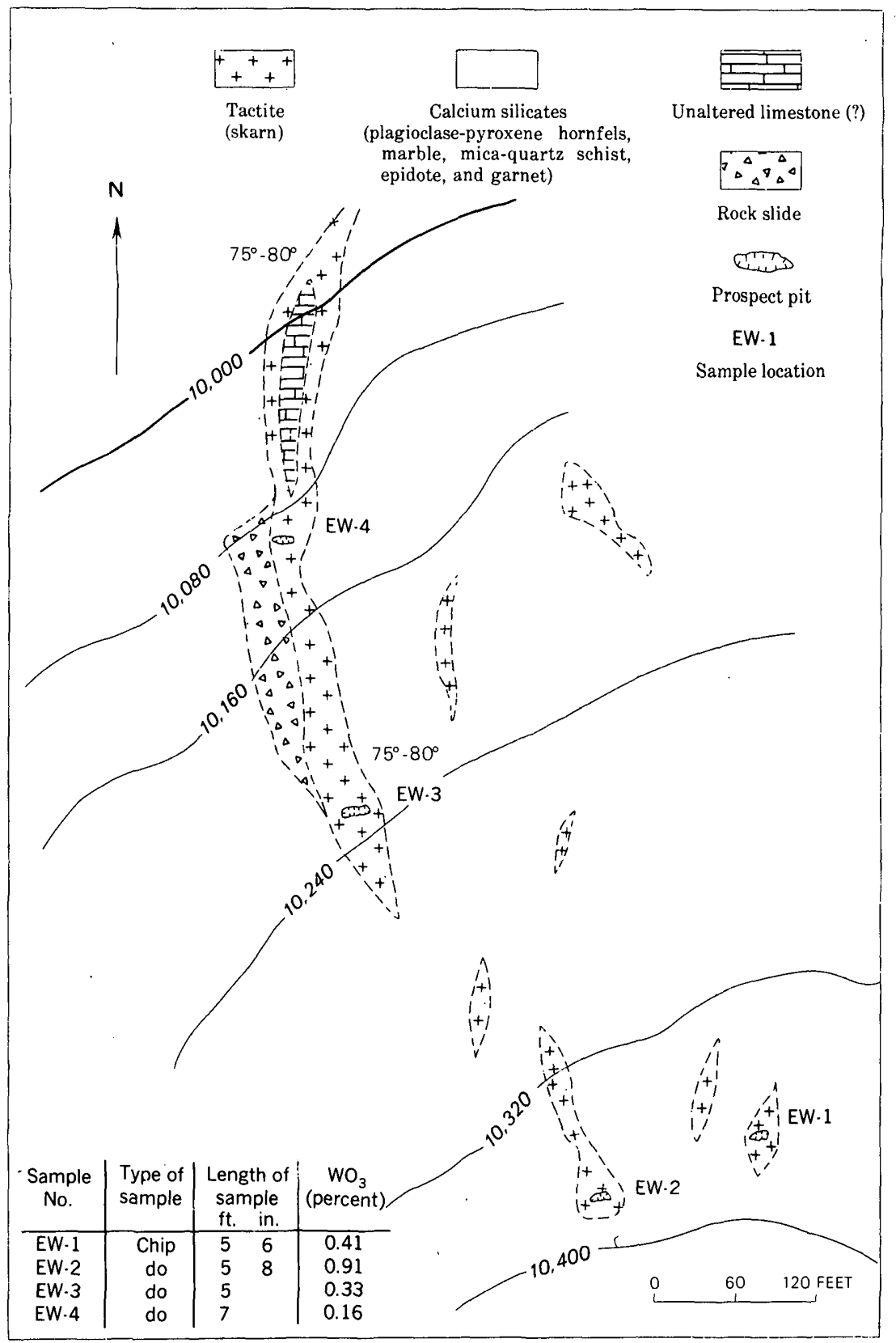




\section{BUCKSKIN GROUP}

The Buckskin and the Buckskin Nos. 1 through 7 claims were located in July 1966 and July 1967 by Emmett L. Dahl. The claims lie in sec. 26, T. 4 N., R. 21 E., west of the Bigelow claims and about 0.5 mile north of Bigelow Peak along a trail to Snow Lake. The claims are underlain by interbedded quartz-mica schist, quartzite, and marble which have been crumpled along steeply dipping fold axes. The bedding and schistosity both strike northwestward and dip steeply to the west. Numerous dikes and sills of aplite and pegmatite have intruded the metamorphosed sedimentary rocks, and a large mass of granitic rock is exposed west of the claims. The principal ore minerals observed in the area are scheelite and molybdenite. These minerals occur along fracture zones in tactite, but the discovery pit on the Buckskin claim also contains pyrite, ferroan sphalerite, and chalcopyrite.

In the shallow discovery pit of the Buckskin claim (fig. 17), a character sample was taken that assayed 0.71 percent $\mathrm{WO}_{3}$. The discovery pit on the north end of Buckskin No. 2 exposes a strong tactite zone striking

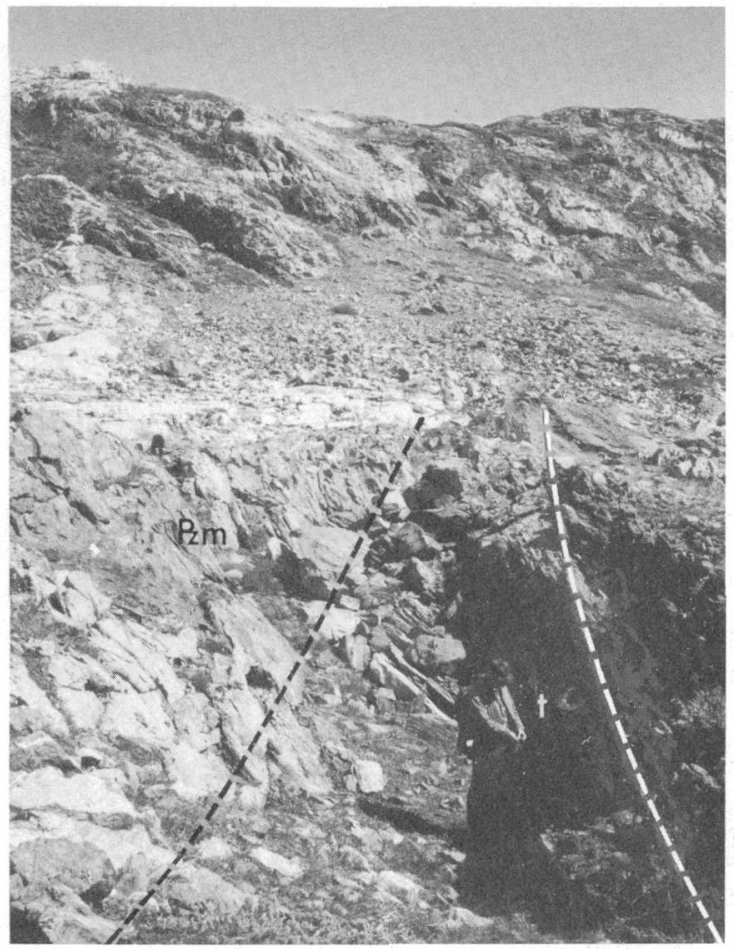

Figure 17.-Discovery pit, Buckskin No. 2 claim. Generalized boundaries of tactite zone $(\dagger)$ enclosed by metasedimentary rocks (Pzm). 
southeastward for 200 feet, where it is cut off by a granitic dike striking southwestward (fig. 18). Character samples of the tactite zone in this pit assayed 0.78 percent $\mathrm{WO}_{3}$.

The small prospect pit on Buckskin No. 2 claim (fig. 19) shows an irregular lens of tactite that is concordant with the steeply dipping marble beds and lenses from which it was derived. A chip sample over a 30 -inch length of this bed assayed 0.1 percent $\mathrm{WO}_{3}$.

No production of tungsten ore has been recorded from the Buckskin group. Although subsurface exploration would be required to determine the extent and grade of the ore bodies to depth, the claims appear to have a limited potential to produce tungsten ore.

\section{ELEVEN KIDS CLAIM}

The 11 Kids claim was located in August 1954, in sec. 25, T. 4 N., R. 21 E., by F. W. Hanson. The claim lies about a quarter of a mile east of Snow Lake at an elevation of 9,500 feet and contains small lenses and thin interbeds of limestone that have been metamorphosed to tactite Most of the scheelite observed in the claim is sparsely disseminated along irregular fractures. The tactite zones are not particularly rich in tungsten and appear to contain only small pods or thin fracture coatings of scheelite. No exploratory cuts or development workings were observed on the claim. No tungsten ore has been produced, and it is believed that the claim has little or no potential for production of such ores.

\section{SNOW LAKE CLAIMS}

The Snow Lake, Snow Lake No. 1, and Snow Lake No. 2 claims are in sec. 25, T. 4 N., R. 21 E., along the south and east sides of Snow Lake, south of the Montezuma mine; they were located by Emmett I. Dahl in September 1956. The property lies on the north side of the roof pendant on a thick domelike section of complexly folded metamorphic rocks locally intruded by dikes and irregular masses of granitic rocks. The scheelite-bearing tactite zones observed during the field studies are small and highly discontinuous. No exploratory or development work has been carried out, and this group of claims does not appear to have high potential for significant production of tungsten ore.

\section{MINERAL POTENTIAL OF THE PRIMITIVE AREA}

The Bigelow Peak roof pendant contains all of the known mineral deposits of any significance within the Emigrant Basin primitive area. Other metamorphic rocks in the area are virtually barren of ore minerals. The known ore bodies occur chiefly in lenses of marble and limy tactite, most of which are small, scattered, and discontinuous, 


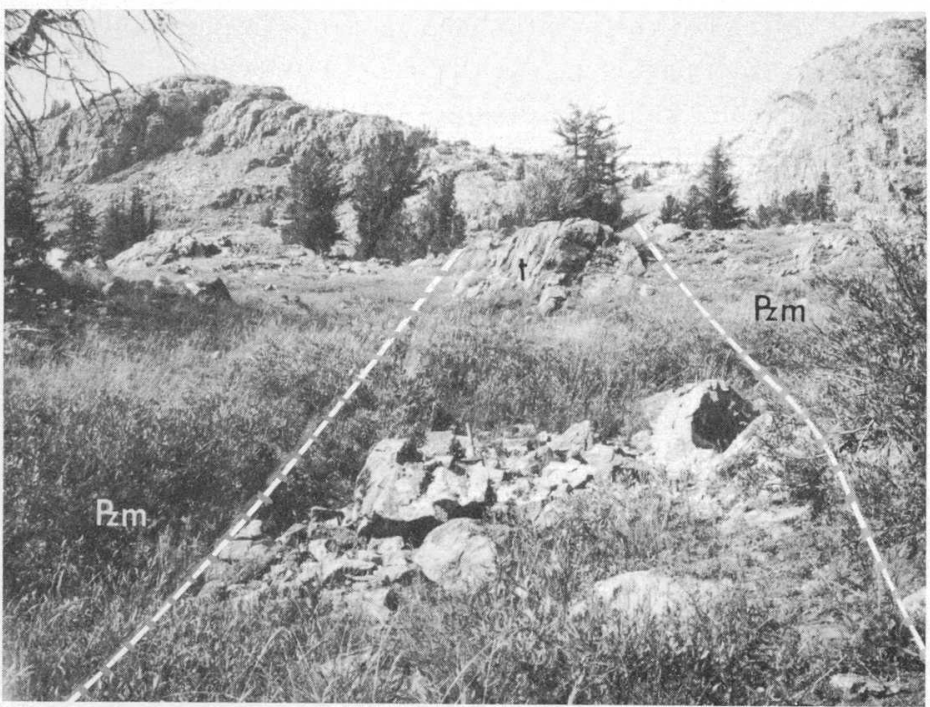

Figure 18.-Tactite zone $(t)$ on the Buckskin claim enclosed by metasedimentary rocks (Pzm).

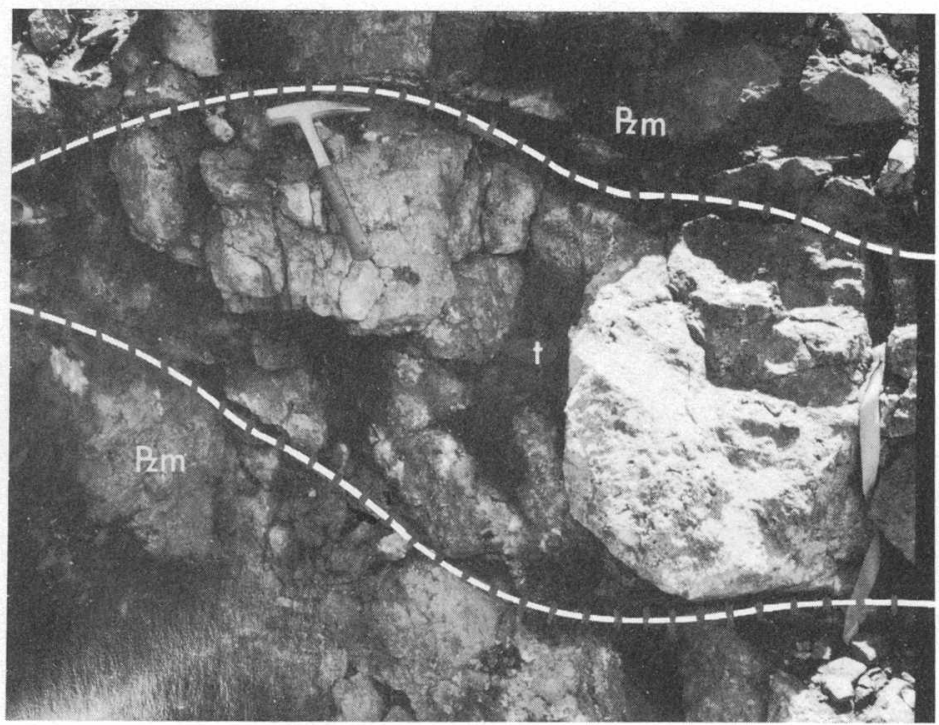

Figure 19.-Face of prospect pit on Buckskin No. 2 claim. Metasedimentary rocks (Pzm) enclose a distinct lens of tactite $(t)$. 
and largely restricted to the northern part of the pendant. Several of the deposits in the tactites are locally rich; however, they appear to be limited in size. The lack of adequate exploration and mine development at depth precludes accurate estimates of the ore reserves. Nevertheless, it may be concluded that a number of ore deposits of possible commercial interest are scattered through the roof-pendant rocks and that discoveries of other ore bodies may be expected in the future, particularly concealed deposits.

The metamorphic septum rocks exposed in the Toms Canyon area do not appear from geologic, geochemical, or geophysical evidence to be mineralized to the same extent as the rocks in the Bigelow Peak pendant. The main difference between these bodies is the absence in the septum of carbonate rocks and tactites and a more advanced state of metamorphism and granitization.

Other mineral deposits, which may reasonably be expected to occur in geologic environments similar to the geologic terrane of the Emigrant Basin area, include (1) fossil placer deposits in sands and gravels preserved beneath the earliest volcanic rocks, (2) hydrothermal ore bodies localized by faults, fissures, and other structures, (3) hydrothermal or epigenetic ore bodies replacing plant debris in the prevolcanic and basal volcanic tuffis and breccias, and (t) bulk deposits of road metal, fill materials, and decorative stone.

Concerning the possible presence of fossil placers, the apparent absence of prevolcanic ore deposits containing gold, tin, platinum, and gem stones in the granitic and metamorphic rocks of the Emigrant Basin area virtually precludes such deposits at the base of the volcanic series. As indicated on page G22, the buried placers of the Mother Lode belt are 30 to 50 miles west of Emigrant Basin and are not known to extend far beyond the lode deposits of the gold belt. Conceivably, scheelite might occur in placer accumulations in the Emigrant Basin area, but none was. detected, neither in geochemical samples of the conglomerate and sandstone at the base of the volcanic rocks, nor the surficial deposits derived from them.

Similarly, the general sparseness of surficial deposits in the icescoured terrane of the Emigrant Basin primitive area, as well as the small size of the known ore deposits that might have been the source of valuable detrital minerals, virtually eliminates the possibility that placer deposits of commercial size and grade are present in the unconsolidated deposits of Holocene age in this region. A possible exception might be found in the alluvial deposits in the drainage basin of the East Fork of Cherry Creek, where a few grains of detrital scheelite were observed in panned concentrates of the stream and lake sediments in Horse Meadow. Similar scheelite occurrences may also be present in 
the alluvium in Summit Meadow and at the head of Huckleberry Iake, but the probable low grade of these and other possible placer deposits would be a critical factor in their commercial exploitation.

Hydrothermal ore bodies in the granitic and rolcanic rocks of the Emigrant Basin area are also either nonexistent or small and have no commercial interest. The linear altered zolie near the head of Cherry Lake in the E1/2 sec. 8 and W1/2 sec. 9, T. 2 N., R. 19 E., Jacks any significant quantities of ore minerals and apparently is not associated with a concealed ore body. As indicated on page G51, the vein on Silver Mine Creek, beyond the limits of the primitive area, is localized by a sheeted zone in the granitic rocks of the Topaz Lake pluton, but this vein is not sufficiently large or rich to warrant further development. No mineral production has been recorded from it. It is conceivable that small hydrothermal ore bodies may occur in the volcanic rocks, but the small size of the altered areas in these rocks indicates that the ore bodies also would be small. On the basis of this evidence, it is concluded that the granitic and volcanic rocks do not contain hydrothermal deposits of interest to the miner or prospector.

A somewhat better chance exists for the occurrence of concentrations of uranium and copper minerals in the basal clastic units of the eruptive rocks, but no geochemical or geophysical indications of such ore deposits have been recognized within the primitive area.

The deposits of road metal, fill materials, decorative stone, and other bulk commodities in the Emigrant Basin area are so inaccessible, and so similar to more readily available deposits of better quality, that they are considered to have no commercial value.

\section{ECONOMIC APPRAISAL}

Although the parts of the Emigrant Basin area that are underlain by granitic and volcanic rocks are virtually devoid of mineral deposits, a small sporadic production of tungsten ore hạs been recorded from a relatively limited region in the southeastern part of the area. Small deposits of tungsten are known and others are inferred to be present on a number of claims. The inferred deposits are probably comparable in grade to ore produced to date, and shipments of ore could and probably will be made in the years to come. Prospecting and exploration for: tungsten in this area might disclose additional tungsten deposits or downward extensions of known deposits.

Because of the similarity of the tungsten ores in the known deposits in the 8 square mile area of the Bigelow Peak pendant, a single custom gravity concentrating plant would be adequate to accommodate the various tungsten properties and probably would permit more economic operations of the prospects. Copper, molybdenum, and lesser quanti- 
ties of lead and zinc are associated with the tungsten deposits, and a differential flotation milling method would permit recovery of these byproducts. Either of these two types of mills would be warranted only if sufficient ore of adequate grade could be proven to be available.

\section{REFERENCES CITED}

Bateman, P. C., 1956, Economic geology of the Bishop tungsten district, California : California Div. Mines Spec. Rept. 47, $87 \mathrm{p}$.

Bateman, P. C., 1965, Geology and tungsten mineralization of the Bishop district, California, with a section on Gravity study of Owens Valley by L. C. Pakiser and M. F. Kane, and a scction on Seismic profile by L. C. Pakiser : U.S. Geol. Survey Prof. Paper 470, 208 p.

Bateman, P. C., Clark, L. D., Huber, N. K., Moore, J. G., and Rinehart, C. D., 1963, The Sierra Nevada batholith-a synthesis of recent work across the central part: U.S. Geol. Survey Prof. Paper 414-D, p. D1-D46.

Bateman, P. C., and Eaton, J. P., 1967, Sierra Nevada batholith : Science, v. 158, no. 3807, p. 1407-1417.

Bateman, P. C., and Wahrhaftig, Clyde, 1966, Geolegy of the Sierra Nevada, in Bailey, E. H., ed., Geology of northern California: California Div. Mines and Geology Bull. 190, p. 107-172.

Calkins, F. C., 1930, The granitic rocks of the Yosemite region, in Matthes, F. E., Geologic history of the Yosemite Valley : U.S. Geol. Survey Prof. Paper 160, p. $120-129$.

Clark, W. B., 1966, Economic mineral deposits of the Sierra Nevada, in Bailey, E. H., ed., Geology of northern California: California Div. Mines and Geology Bull. 190, p. 209-214.

Davis, L. E., 1967a, The mineral industry of California, in U.S. Bur. Mines, Minerals Yearbook, 1965: Washington, D.C., U.S. Govt. Printing Office, v. 3, p. 133-169.

- 1967b, The mineral industry of California, in U.S. Bur. Mines, Minerals Yearbook, 1966: Washington, D.C., U.S. Govt. Printing Office, v. 3, p. 133169.

Dodge, F. W. C., and Fillo, P. V., 1967, Mineral resources of the Desolation Valley primitive area of the Sierra Nevada, California: U.S. Geol. Survey Bull. 1261-A, p. A1-A27.

Dubois, R. I., 1963, Remanent, induced, and total magnetism of a suite of serpentine specimens from the Sierra Nevada, California: Jour. Geophys. Research, v. 68, no. 1, p. 267-278.

Grimes, D. J., and Marranzino, A. P., 1968, Direct-current arc and alternatingcurrent spark emission spectographic field methods for the semiquantitative analysis of geologic materials : U.S. Geol. Survey Circ. 591, 6 p.

Grommé, C. S., and Merrill, R. T., 1965, Paleomagnetism of Late Cretaceous granitic plutons in the Sierra Nevada, California-further results: Jour. Geophys. Research, v. 70, no. 14, p. 3407-3420.

Henderson, J. R., Jr., Stromquist, A. A., and Jespersen, Anna, 1966, Aeromagnetic map of parts of the Mother Lode Gold and Sierra Foothills copper mining district, California, and its geologic interpretation: U.S. Geol. Survey Geophys. Inv. Map GP-561, scale 1: 62,500.

Huffman, Claude, Jr., Mensik, J. D., and Riley, L. B., 1967, Determination of gold in geologic materials by solvent extraction and atomic-absorption spectrometry : U.S. Geol. Survey Circ. 544, 6 p. 
Jenkins, O. P., director, and others, 1949, The counties of California-mineral resources and mineral production during 1947 : California Div. Mines Bull. $142,197 \mathrm{p}$.

Koenig, J. B., 1963, Geologic map of California, Olaf P. Jenkins edition, Walker Lake sheet: California Div. Mines and Geology, scale $1: 250,000$.

Krauskopf, K. B., 1953, Tungsten deposits of Madera, Fresno, and Tulare Counties, California : California Div. Iines and Geology Spec. Rept. 35, 83 p.

Lemmon, D. M., Bateman, P. C., Stagner, H. R., and Beatty, M. E., 1943, Tungsten deposits near Dorothy Lake, Yosemite National Park, Tuolumne County, California. U.S. Geol. Survey Strategic Minerals Inv. Prelim. Map [open-file report, August 30, 1943], 2 sheets.

Little, J. M., 1942, Tungsten deposits of the Confidence mining district, Tuolumne County, California : California Jour. Mines and Geology, v. 38, nos. 3 and 4, p. 283-290.

McLaughlin, R. P., 1907, Geology of the Bodie district, Californila : Mining and Sci. Press, v. 94, p. 795-796.

Matthes, F. E., 1930, Geologic history of Yosemite Valley : U.S. Geol. Survey Prof. Paper $160,137 \mathrm{p}$.

Peterson, D. W., Yeend, W. E., Oliver, H. W., and Mattick, R. E., 1968, Tertiary gold-bearing channel gravel in northern Nevada County, California: U.S. Geol. Survey Circ. 566, $22 \mathrm{p}$.

Ransome, F. L., 1898, Some lava flows of the western slope of the Sierra Nevada, California : U.S. Geol. Survey Bull. 89, $74 \mathrm{p}$.

Rensch, H. E., Rensch, E. G., and Hoover, M. B., 1933, Historic spots in California, valley and Sierra counties: Stanford, Calif., Stanford Univ. Press, p. 502-503.

Slemmons, D. B., 1966, Cenozoic volcanism of the central Sierra Nevada, California, in Bailey, E. H., ed., Geology of northern California : California Div. Mines and Geology Bull. 190, p. 199-208.

Smith, A. R., 1968, Petrography of six granitic intrusive units in the Yosemite Valley area, California: California Div. Mines and Geology Spec. Rept. 91, p. 3-15.

Thompson, C. E., Nakagawa, H. M., and VanSickle, G. H., 1968, Rapid analysis for gold in geologic materials, in Geological Survey research, 1968: U.S. Geol. Survey Prof. Paper 600-B, p. B130-B132.

Tucker, W. B., and Sampson, R. J., 1941, Mono County, in Recent developments in the tungsten resources of California : California Jour. Mines and Geology. v. 37 , no. 4 , p. 581 .

Turekian, K. K., and Wedepohl, K. H., 1961, Distribution of the elements in some major units of the earth's crust: Geol. Soc. America Bull., v. 72, no. 2, p. 175-191.

U.S. Coast and Geodetic Survey, 1965, Total intensity chart of the United States, 1965.0 : U.S. Coast and Geod. Survey Chart $3077 \mathrm{f}$.

Vacquier, Victor, Steenland, N. C., Henderson, R. G., and Zietz, Isidore, 1951, Interpretation of aeromagnetic maps: Geol. Soc. America Mem. 47, $151 \mathrm{p}$.

Vaughn, W. W., 1967, A simple mercury vapor detector for geochemical prospecting: U.S. Geol. Survey Circ. 540, 8 p.

Wahrhaftig, Clyde, 1962, Geomorphology of the Yosemite Valley region, California, in Oakeshott, G. B., ed., Geologic guide to the Merced Canyon and Yosemite Valley, California: California Div. Mines and Geology Bull. 182, p. 33-46. 
Ward, F. N.; Lakin, H. W., Canney, F. C., and others, 1963, Analytical methods used in geochemical exploration by the U.S: Geological Survey: U.S. Geol. Survey Bull. 1152, $100 \mathrm{p}$.

Zietz, Isidore, and Kirby, J. R., 1968, Transcontinental geophysical survey $\left(35^{\circ}-39^{\circ} \mathrm{N}\right)$ magnetic map from $112^{\circ} \mathrm{W}$ longtitude to the coast of California: U.S. Geol. Survey Misc. Geol. Inv. Map I-532A, scale 1: 1,000,000. 